\title{
CHARACTERIZATION OF THE GEOCHEMICAL AND PHYSICAL PROPERTIES OF WETLAND SOILS ON THE SAVANNAH RIVER SITE: FIELD SAMPLING ACTIVITIES (U) FINAL REPORT
}

by WSRC Contact - K. L. Dixon

Westinghouse Savannah River Company

Savannah River Site

Aiken, South Carolina 29808

Other Authors:

Metcalf \& Eddy
WSRC-TR--92-576

DE93 006793

This paper was prepared in connection with work done under Contract No. DE-AC09-89SR18035 with the U.S. Department of Energy. By acceptance of this paper, the publisher and/or recipient acknowledges the U. S. Government's right to retain a nonexclusive, royalty-tree license in and to any copyright covering this paper, along with the right to reproduce and to authorize others to reproduce all or part of the copyrighted paper. 


\section{DISCLAIMER}

This report was prepared as an account of work sponsored by an agency of the United States Government. Neither the United States Government nor any agency thereof, nor any of their employees, makes any warranty, express or implied, or assumes any legal liability or responsibility for the accuracy, completeness, or usefulness of any information, apparatus, product, or process disclosed, or represents that its use would not infringe privately owned rights. Reference herein to any specific commercial product, process, or service by trade name, trademark, manufacturer, or otherwise does not necessarily constitute or imply its endorsement, recommendation, or favoring by the United States Government or any agency thereof. The views and opinions of authors expressed herein do not necessarily state or reflect those of the United States Government or any agency thereof.

This report has been reproduced directly from the best available copy.

Available to DOE and DOE contractors from the Office of Scientific and Technical Information, P.O. Box 62, Oak Ridge, TN 37831; prices available from (615) 576-8401, FTS 626-8401.

Available to the public from the National Technical Information Service, U.S. Department of Commerce, 5285 Port Royal Rd, Springfield, VA 22161. 
Submitted to:

Westinghouse Savannah River Company

Aiken, South Carolina

\section{Characterization of the Geochemical and Physical Properties of Wetland Soils on the Savannah River Site: Field Sampling Activities}

\section{Final Report}

Contract No. AA46327P

Task No. 30

November 1992




Submitted to:

Westinghouse Savannah River Company

Aiken, South Carolina

\section{Characterization of the Geochemical and \\ Physical Properties of Wetland Soils on the \\ Savannah River Site: Field Sampling Activities}

\section{Final Report}

Contrect No. AA46327P

Task No. 30

November 1992




Table of Contents

INTRODUCTION $\ldots \ldots \ldots \ldots \ldots \ldots \ldots \ldots \ldots \ldots \ldots \ldots \ldots \ldots \ldots \ldots \ldots \ldots$

OBJECTTVES $\ldots \ldots \ldots \ldots \ldots \ldots \ldots \ldots \ldots \ldots \ldots \ldots \ldots \ldots \ldots \ldots \ldots \ldots$

METHODS, PROCEDURES AND MATERIALS $\ldots \ldots \ldots \ldots \ldots \ldots \ldots \ldots$





Appendix A - Core Logs

Appendix B - Field Activities Logs

Appendix C - Chain of Custody Forms

\section{List of Tables}



Table 2. Sampling Intervals for Each Soil Grouping $\ldots \ldots \ldots \ldots \ldots \ldots \ldots \ldots$

Table 3. Particle Size Breakdown Used In Lithologic Logging $\ldots \ldots \ldots \ldots \ldots$

Table 4. Summary of Field Data for Off-site Vibracores $\ldots \ldots \ldots \ldots \ldots \ldots \ldots \ldots$

Table 5. Soil Groupings Encountered in Off-site Vibracores $\ldots \ldots \ldots \ldots \ldots \ldots$

Table 6. Locations and Sample Numbers of Duplicate Soil Cores . . . . . . . . . . . . . 12

Table 7. Summary of Field Data for On-site Vibracores $\ldots \ldots \ldots \ldots \ldots \ldots \ldots$

Table 8. Soil Groupings Encountered in On-site Vibracores $\ldots \ldots \ldots \ldots \ldots \ldots$ 


\section{INTRODUCTION}

There are 36,000 acres of wetlands on the Savannah River Site (SRS) and an additional 5,000 acres of floodplain. Recent studies of wetland soils near various waste sites at SRS have shown that some wetlands have been contaminated with pollutants resulting from SRS operations. In general, releases of contaminants to wetland areas have been indirect. These releases may have originated at disposal lagoons or waste lacilities located in the vicinity of the wetland areas. Transport mechanisms such as surface runoff, soil erosion, sediment transport, and groundwater seepage into downgradient wetland areas are responsible for the indirect discharges to the wetland areas.

The SRS determined that a database of background geochemical and physical properties for wetland soils on the SRS was needed to facilitate future remedial investigations, human health and ecological risk assessments, treatability studies, and feasibility studies for the wetland areas. These data are needed for comparison to contaminant data collected from wetland soils that have been affected by contamination from SRS operations. This report describes the efforts associated with the collection of soil cores, preparation of a lithologic log for each core, and the processing and packaging of individual soil samples for shipment to analytical laboratory facilities.

\section{OBJECTIVES}

The objectives of this field study are listed below:

1. To collect a total of eighty-three, 10 foot long vibracore soil samples. These eightythree vibracore samples were to be collected from 50 locations on the SRS and 25 locations off-site within a 100 miles of the SRS.

2. To divide each of these cores into individual soil samples according to soil horizons.

3. To package each individual soil sample and send it to a WSRC subcontracted 
laboratory to be analyzed for the following contaminants and properties: RCRA Appendix IX constituents, radionuclides, pesticides and PCBs, nutrients, and other agricultural and physical parameters.

4. To prepare a lithologic description for each soil core locatisn.

5. To complete all activities in accordance with an approved task specific Quality Assurance Project Plan.

\section{METHODS, PROCEDURES AND MATERIALS}

The wetlands on the SRS are composed of nine different soil types that vary significantly in terms of their geological and hydrogeological characteristics. Due to the large number of soil types, soils were combined into groupings that were similar in terms of their geochemical and physical characteristics. Three broad categories were used to combine the soil groups: soils occurring in small stream floodplains, soils occurring in upland bays and depressions, and soils occurring in large stream (Savannah River) floodplains. Five soil groupings were selected for the sampling as listed in Table 1.

The collection of these soil groupings was not limited to SRS. Approximately one third of the entire project was conducted off-site. The off-site sampling was intended to represent unimpacted study sites (as related to SRS) for each of the five soil groupings. Each of the off-site sampling locations were in South Carolina. The off-site sampling locations were: Aiken State Park, Aiken; Audubon Society, Jackson; Congaree Swamp National Monument, Gadsden; Francis Beidler Forest, Harleyville; and Santee State Park, Santee.

The vibracore sampling technique was used to collect ten foot long, three inch diameter cores. A total of 28 cores at 25 study sites were taken off-site during late July and early August of 1992. Five cores were collected from each of the five soil groupings with three duplicate cores. Within the boundary of SRS, a total of 55 cores were collected at 50 study sites, with ten cores from each 
Soil Group

Group One - Dorovan, Other Histosols

Group Two - Pickney, Johnston

Group Three - Fluvaquents

Group Four - Rembert, Ogeechce, Williman

Group Five - Chastain, Tawcaw
Broad Category

Small Stream Floodplain - Organic Soils

Small Stream Floodplain - Intergrade Mineral-Organic Soils

Small Stream Floodplain - Mineral Soils

Upland Bays \& Depressional Soils

Large Stream Floodplain Soils

of the five soil groupings and five duplicate cores, one each from each soil grouping. On-site cores were taken during August and early September of 1992.

Several changes were made in the initial sampling procedures in order to meet field conditions. Initially, plastic core liners were used inside the vibracore sample barrel. The penetration of the sample barrel into the ground was not sufficient when the plastic liner technique was employed; therefore, the plastic liner technique was eliminated, and all vibracore sampling collected during the project utilized an aluminum core barrel.

The initial sampling protocol concerning the number of cores collected at each sampling site was also changed. Once it was determined that a large volume of sample was needed to fill the required sample bottles, two full length (10 foot) vibracores were collected at each sample site. Two shorter hammer cores (30 to 40 inches) were also collected at each sample site to ensure enough sediment volume for the $A$ soil horizon. 
Initially, if the vibracore met refusal in gravelly or clayey materials, the sampling at that site was considered complete. This was amended to ensure a complete sample set for all the core sites. This meant that all sampling would reach 10 feet below the ground surface. This was accomplished by using a bucket hand auger to retrieve samples which could not be collected by the vibracore technique.

Each core was sub-sampled by dividing the core into 5 sub-sections based on general soil horizon changes as defined in Table 2, and compaction. The A and B sample intervals for soils groupings 3,4 , and 5 were determined only after visual inspection of a given core, with their sum total not to exceed 40 inches. Once subdivided, the cores were sampled and packaged. The samples were then shipped by SRS to a laboratory for analytical analysis.

\section{Core Collection}

Cores were collected with minimum disturbance to the wetland areas. Vehicles were not used in the wetlands; all equipment, materials, and cores were back-packed to and from the study sites.

Cores were collected via two methodologies: 1) vibracore; and 2) hand auger. The vibracore sampling methodology consisted of vibrating a three inch diameter aluminum tube down to the maximum sampling depth of ten feet. The cutting edge of the tube was beveled and sharpened to facilitate penetration into the soil profile. Sandy and gravelly soils tended to fall from the end of the tube upon withdrawal; therefore, when possible, the sample tube was vibrated to a depth of eleven or tweive feet to help ensure recovery of the full ten foot interval. After the three inch aluminum tube was vibrated down to the desired depth, the headspace in the core barrel was filled with deionized water. Then the tube was capped with a plumber's test plug. The vacuum prevented slippage of the core from the sample tube during withdrawal. The three inch aluminum tube was then extracted by using a come-along (jack), tripod, and tackle arrangement. Core 
Table 2. Sampling Intervals For Each Soil Grouping

\begin{tabular}{|c|c|c|}
\hline Soil Group & $\begin{array}{l}\text { Sampling Interval } \\
\text { ID Code }\end{array}$ & $\begin{array}{l}\text { Sampling Interval } \\
\text { (in) }\end{array}$ \\
\hline 1 & $\begin{array}{l}\text { A } \\
B \\
C \\
D \\
E\end{array}$ & $\begin{array}{c}0-20 \\
20-40 \\
40-60 \\
60-90 \\
90-120\end{array}$ \\
\hline 2 & $\begin{array}{l}\text { A } \\
B \\
C \\
D \\
E\end{array}$ & $\begin{array}{c}0.20 \\
20-40 \\
40-60 \\
60-90 \\
90-120\end{array}$ \\
\hline 3 & $\begin{array}{l}\text { A } \\
B \\
C \\
D \\
E\end{array}$ & $\begin{array}{c}\text { A Horizon } \\
\text { Bottom of A-40 } \\
40-60 \\
60-90 \\
90-120\end{array}$ \\
\hline 4 & $\begin{array}{l}\text { A } \\
B \\
C \\
D \\
E\end{array}$ & $\begin{array}{c}\text { A Horizon } \\
\text { Bottom of A-40 } \\
40-60 \\
60-90 \\
90-120\end{array}$ \\
\hline 5 & $\begin{array}{l}\text { A } \\
B \\
C \\
D \\
E\end{array}$ & $\begin{array}{c}\text { A Horizon } \\
\text { Bottom of A-40 } \\
40-60 \\
60-90 \\
90-120 \\
\end{array}$ \\
\hline
\end{tabular}


penetration and core compaction was recorded prior to core extraction. The resulting hole created by the removal of the core was backfilled with soil obtained from the sampling area.

At most of the vibracoring sites, two ten foot long cores were collected. This was necessary due to the volume of soil needed to fill the sample jars for each of the five sub-sample horizons. For samples collected by auger, the volume of material obtained from the ten foot core was sufficient. Also in soil groupings 3, 4, and 5 which occasionally had thin A horizons, two additional push/hammer cores were collected to provide additional A horizon soil material. All collected cores were maintained at $4^{\prime \prime} \mathrm{C}$ for transport from the field to the laboratory facility provided by SRS.

When vibracore sampling was not possible, due mainly to clayey or gravelly soils, a $31 / 4$ inch stainless steel bucket auger was used. All soil collected with the auger was placed in plastic bags, sealed, and labeled according to station ID, and soil horizon/depth. Volatile samples were collected at the sample site immediately upon removal of the soil via the bucket auger, and kept at $4^{\circ} \mathrm{C}$ for transport to the lab. Rubber gloves were used while sampling the auger, and were changed between intervals to prevent cross-contamination. When flowing sands were encountered during the sampling, the bucket auger technique was replaced with the vibracore in order to recover the remaining sample.

Of the 75 coring sites, portions of 16 cores were hand augered. Only one sample site was not completed to the designated depth of ten feet. At sample site BGW050, large gravel (pebbles and cobbles) was encountered in the $\mathrm{E}$ horizon which was impenetrable using the vibracore technique. Other attempts were made to complete the core (including use of hand auger and general excavation). Sample E was not retained because the sample consisted of 95 percent pebbles and cobbles and 5 percent coarse sand.

A field activities log was completed for each coring site. At each coring site, a Global Positioning Systems (GPS) reading of latitude and longitude was taken and recorded in the field activities log. 
The GPS location readings are reported in Appendices $A$ and $B$ in degrees and minutes to the nearest hundredth of a minute.

\section{Core Opening and Sub-Sampling}

After a core was transported to SRS (Building 704-B), it was cut longitudinally using a radial saw with a decontaminated carbide-tipped saw blade. The core was cut in such a manner to minimize disturbance to the core sample (the cutting of the aluminum tube only). Upon opening the tube, each core was divided into five sub-samples. If two cores were collected at a station, they were designated Core A and Core B. In most cases Core A was logged and sampled and additional soil material was taken from Core $B$ if necessary. In the event that Core $B$ had decfer penetration, more complete recovery, and less compaction, Core B was sampled and logged. All intervals were corrected for compaction, which was averaged over the entire length of the core sample. Example compaction calculations are provided in A.ppendix A. If a given core required sampling for volatile organic compounds this was accomf ned immediately after opening of the core. The volatile sample was collected trom the middle of the core sample. Once all the volatile samples were collected, they were placed in a bag and stored in a refrigerator. Prior to shipment, a trip blank was labeled and placed in the bag with the volatile samples. Trip blanks consisted of presealed bottles of deionized water sent from Weston Laboratories.

To reduce aluminum contamination during the sampling procedure, the outer surface of the core sediment was scraped to remove any aluminum chips resulting from the core opening process. Each interval was then removed from the core barrel and placed into a decontaminated stainless steel mixing pan. The sample was then thoroughly agitated with a decontaminated stainless steel spoon to ensure complete homogenization. Sub-samples were divided into quadrants for filling the sample jars. Each jar was filled with soil taken from one quadrant. After four jars were filled, the remaining soil was homogenized and divided again. This procedure was repeated until all sample jars had been collected. All sample jars were sealed, labeled and placed into a refrigerator until packaged for shipment. 


\section{Lithologic Logging}

For each core location a complete lithologic log was prepared for the entire ten foot sampling depth plus any additional core that may have been collected at a given site. Detailed core logs are presented in Appendix A. The cores were !ugged from a sedimentological perspective. This included a breakdown of significant lithologic changes, color, sediment composition (gravel, sand, mud), organic content, and rooting. The logs provide a graphic image of the core and the general sedimentological charavter. Samples were collected from each interval for grain size or particle size analysis; this will provide data for use in conjunction with the logs when analyzing the laboratory results. The general particle breakdown is presented in Table 3.

Table 3. Particle Size Breakdown Used In Lithologic Logging

Size Class

Gravel -

Cobble

Pebble

Granule

Sand -

Very Coarse and Coarse

Medium

Fine and Very Fine

Mud -
Silt and clay sized sediments

\section{Size Range}

$25.6 \mathrm{~cm}-6.4 \mathrm{~cm}$

$6.4 \mathrm{~cm}-0.4 \mathrm{~cm}$

$0.4 \mathrm{~cm}-0.2 \mathrm{~cm}$

$0.2 \mathrm{~cm}-0.05 \mathrm{~cm}$

$0.05 \mathrm{~cm}-0.025 \mathrm{~cm}$

$0.025 \mathrm{~cm}-0.00625 \mathrm{~cm}$

Finer than $0.00625 \mathrm{~cm}$.

Grain size comments on the lithologic logs were derived from visual inspection based on the above size class breakdowns. The organic content of the cores was viewed closely in order not to confuse a well decomposed peat (Sapric) with a mud. 


\section{Sampling Equipment Decontamination}

All equipment (i.e. sample tubes, sample trays, sample spoons, saw blades) in contact with the core was decontaminated in the following manner.

1. Washed to remove heavy soil or clay. All aluminum sample tubes were ste? cleaned.

2. Hand washed in a phosphate-free detergent (Alconox).

3. Rinsed thoroughly with tap water.

4. Rinsed with deionized water.

5. Rinsed with isopropyl alcohol, air dry.

6. Rinsed again with deionized water. Air dried away from heavy dust areas.

All excess isopropyl alcohol was collected in a five gallon bucket and allowed to evaporate. The sample pans, sample spoons, and saw blades were then wrapped in aluminum foil with the shiny side away from the equipment. The aluminum sample tubes were wrapped in plastic and opened only at the sample site where used.

Two rinsate samples were collected during the off-site sampling. After all sampling pans and spoons had been properly decontaminated and were ready for sampling, a pan was selected randomly and filled with deionized water. This water was poured into the two rinsate bottles and refrigerated until shipment to the laboratory.

\section{Sample Packing for Shipment}

All packaging of the samples met the DOE/DOT shipping requirements. Only insulated coolers were used. Samples were packed one core per cooler for the duration of the project. Each sample interval was packaged and sealed in its own separate plastic bag, which fit into a second larger plastic bag which was sealed around all the samples in the cooler. Vermiculite was used for 
packing the coolers to protect and help insulate sample jars during shipment. Blue ice was included with all of the shipments to ensure that the samples remained cooled during the shipment. Once packaged, the cooler was transported to SRS shipping/receiving along with a chain of custody form and shipping order. As part of the shipping process, the coolers were received by shipping/receiving, shipping tags removed, and chain of custody forms signed. The last step was the placement of a custody seal on the front and back lid of each cooler.

\section{RESULTS}

Data sheets for collection, sampling and packaging of the cores are provided in Appendices A, B, and C. Lithologic logs are presinted in Appendix A. These logs present the geologic character of the core locations and give a dexailed breakdown of the sub-sample intervals used for the core accounting for wmpaction. The sample intervais are shown for both of the cores taken at a given site. The field activities logs are shown in Appenaix B. These logs contain information recorded during the actual coring and are an important supplement to the lithologic logs in Appendix A. Recorded on these data sheets are the date and time the core was collected; weather conditions; personnel; core penetration; recovery and compaction; and other notes and comments pertinent to each location. Appendix C contains the chain of custody forms for samples provided to SRS for shipment to the laboratory for analysis. Each chain of custody form represents a cooler and the samples it contained.

\section{Off-Site Sampling (July - August 1992)}

A summary of the core data is presented in Tables 4 and 5 for the off-site sample collection. These tables represent the essential data for each of the 25 coring sites. Table 4 outlines when the sample site was cored, sub-sampled, and shipped as well as information on total core penetration, recovery, and compaction for the individual core (either Core A or Core B) that was logged and sampled. Table 5 presents the location from which the core was taken and the soil grouping. The three QS coring stations represent duplicate cores collected at the three stations indicated in Table 6. 
Table 4. Summary of Field Data for Off-site Vibracores

\begin{tabular}{|c|c|c|c|c|c|c|}
\hline & Date & Date & Date & & Field Restalts & \\
\hline $\begin{array}{c}\text { Vibracore } \\
\text { Station } \\
\end{array}$ & $\begin{array}{c}\text { Core } \\
\text { Collected }\end{array}$ & $\begin{array}{c}\text { Core } \\
\text { Sampled }\end{array}$ & $\begin{array}{c}\text { Core } \\
\text { Shipped }\end{array}$ & $\begin{array}{c}\text { Core } \\
\text { Penetration } \\
\end{array}$ & $\begin{array}{c}\text { Core } \\
\text { Recovery }\end{array}$ & $\begin{array}{c}\text { Core } \\
\text { Compaction }\end{array}$ \\
\hline BGW001 & $7-28-92$ & $7.28-92$ & $7-29-92$ & $129 "$ & $109^{\prime \prime}$ & 9" \\
\hline BGW002 & $7-28-92$ & $7-29-92$ & $7.29-92$ & $121^{\prime \prime}$ & $97^{11}$ & $25^{\prime \prime}$ \\
\hline BGW003 & $7-29-92$ & $7-30-92$ & $7-30-92$ & $81^{\prime \prime}$ & $67^{\prime \prime}$ & $12^{11}$ \\
\hline BGW004 & $7-29-92$ & $7-30-9$ & $-30-92$ & $138^{\prime \prime}$ & $127.5^{\prime \prime}$ & $14^{\prime \prime}$ \\
\hline BGW005 & $7-27-92$ & $7-27-9$ & $7-28-92$ & $144^{\prime \prime}$ & $87^{\prime \prime}$ & $32^{\prime \prime}$ \\
\hline BGW006 & $7-29-92$ & $\longdiv { - 3 0 - 9 2 }$ & $7-31-92$ & $139^{\prime \prime}$ & $88^{\prime \prime}$ & $3)^{\prime \prime}$ \\
\hline BGW007 & $7-27-92$ & $7-27-92$ & $7-28-92$ & $138^{\prime \prime}$ & $109^{\prime \prime}$ & $26^{\prime \prime}$ \\
\hline BGW008 & $7-29-92$ & $7-30-92$ & $7-31-92$ & $132^{\prime \prime}$ & $120^{\pi}$ & $6.5^{\prime \prime}$ \\
\hline BGW009:* & $8-03-92$ & $8-05-92$ & $8-07-92$ & $144^{\prime \prime}$ & $106^{\prime \prime}$ & $35^{\prime \prime}$ \\
\hline BGW010 & $8-02-92$ & $8-03-92$ & $8-05-92$ & $143^{\prime \prime}$ & $118^{\pi}$ & $24^{\prime \prime}$ \\
\hline BGW011 & $8-02-92$ & $8-03-92$ & $8-05-92$ & $135^{\prime \prime}$ & $\ln 31$ & $30^{\prime \prime}$ \\
\hline BGW012* & $8-02-92$ & $8-03-92$ & $8-05-92$ & $27^{\pi}$ & $27^{\prime \prime}$ & $0^{\pi}$ \\
\hline BGW013 & $8-03-92$ & $8-05-92$ & $8-06-92$ & $62^{\prime \prime}$ & $57^{\prime \prime}$ & $5 "$ \\
\hline BGW014 & $8-0492$ & $8-06-92$ & $8-07-92$ & $92 \pi$ & $87^{\prime \prime}$ & $5^{\pi}$ \\
\hline BGW015* & $8-04-92$ & $8-06-92$ & $8-07-92$ & $70^{\prime \prime}$ & $56^{\prime \prime}$ & $3^{\prime \prime}$ \\
\hline BGW016* & $8-04-92$ & $8-06-92$ & $8-07-92$ & $101^{\prime \prime}$ & $99^{\prime \prime}$ & $1^{11}$ \\
\hline BGW017* & $8.04-92$ & $8-06-92$ & $8-07.92$ & $58 "$ & $45^{\prime \prime}$ & $14^{\prime \prime}$ \\
\hline BGW018 & $8-03-92$ & $8.05-92$ & $8-06-92$ & $142^{\prime \prime}$ & $121^{\prime \prime}$ & $19^{\prime \prime}$ \\
\hline BGW019 & $7-30-92$ & $7.31-92$ & $8-03.92$ & $116^{\prime \prime}$ & $106^{\prime \prime}$ & $4^{\prime \prime}$ \\
\hline BGW020 & $7-30-92$ & $7-31-92$ & $7-31-92$ & $116^{11}$ & $110^{\prime \prime}$ & $6^{\prime \prime}$ \\
\hline BGW021 & $7-22-92$ & $7-22-92$ & $7-23.92$ & $153^{\prime \prime}$ & $99^{\prime \prime}$ & $52^{\prime \prime}$ \\
\hline BGW022 & $8-06-92$ & $8=07-92$ & 8.07 .92 & $156^{\prime \prime}$ & $115^{\prime \prime}$ & $35^{\prime \prime}$ \\
\hline BGW023 & $7-23-92$ & $7-23-92$ & $7-24-92$ & $132^{\prime \prime}$ & $100^{\prime \prime}$ & $32^{\prime \prime}$ \\
\hline BGW024 & $7-22-92$ & $7-23-92$ & $7-23-92$ & $132^{\prime \prime}$ & $124^{\prime \prime}$ & $7^{11}$ \\
\hline BGW025 & $7-23-92$ & 7.24 .92 & $7-24-92$ & $132^{\prime \prime}$ & $103.5^{11}$ & $28^{\prime \prime}$ \\
\hline BGWIQS & $7-30-92$ & $7-31-92$ & $7-31-92$ & $120^{\prime \prime}$ & $95^{\prime \prime}$ & $24^{\prime \prime}$ \\
\hline BGW2OS & $8-03-92$ & $8-05.92$ & $8-06-92$ & $144^{\prime \prime}$ & $124^{\prime \prime}$ & $31^{\prime \prime}$ \\
\hline BGW30S & $8-06-92$ & $8=07-92$ & $8-07-92$ & $144^{\prime \prime}$ & $124^{\prime \prime}$ & $16^{\prime \prime}$ \\
\hline
\end{tabular}

(*) sites which required the use of a hand auger to collect sample below vibracore refusal.

(**) sites for which Core B was logged and sampled due to deeper penetration, more complete recovery, and less compaction in Core B 
Table 5. Soil Groupings Encountered in Off-site Vibracores

\begin{tabular}{|c|c|c|c|c|}
\hline \begin{tabular}{|c|} 
Vibracore \\
Station \\
\end{tabular} & $\begin{array}{l}\text { Soil } \\
\text { Group }\end{array}$ & Sample Location & $\begin{array}{c}\text { Latitude } \\
\text { (Degrees-Minutes) }\end{array}$ & $\begin{array}{c}\text { Longitude } \\
\text { (Degrees-Minutes) }\end{array}$ \\
\hline BGW001 & 1 & Aiken State Park & $33-32.84 \mathrm{~N}$ & $81-28.64 \mathrm{~W}$ \\
\hline BGW002 & $\overline{3}$ & Aiken State Park & $33-32.92 \mathrm{~N}$ & $81-28.86 \mathrm{~W}$ \\
\hline BGW003 & $\overline{3}$ & Aiken State Park & $33-33.08 \mathrm{~N}$ & $81-28.96 \mathrm{~W}$ \\
\hline BGW004 & 4 & Aiken State Park & $33-33.28 \mathrm{~N}$ & $81-29.39 \mathrm{~W}$ \\
\hline BGW005 & $T$ & Aiken State Park & $33-33.20 \mathrm{~N}$ & $81-29.26 \mathrm{~W}$ \\
\hline BGW006 & $\bar{T}$ & Aiken State Park & $33-33.26 \mathrm{~N}$ & $81-29.31 \mathrm{~W}$ \\
\hline BGW007 & 3 & Aiken State Park & $33-33.52 \mathrm{~N}$ & $81-29.88 \mathrm{~W}$ \\
\hline BGW008 & 2 & Aiken State Park & $33-33.58 \mathrm{~N}$ & $81-29.18 \mathrm{~W}$ \\
\hline BGW009 & $T$ & Congaree Monument & $33-49.77 \mathrm{~N}$ & $80-49.21 \mathrm{~W}$ \\
\hline BGW010 & 5 & Congaree Monument & $33-49.10 \mathrm{~N}$ & $80-48.81 \mathrm{~W}$ \\
\hline BGW011 & 5 & Congaree Monument & $33-49.56, \overline{8}$ & $80-49.42 \mathrm{~W}$ \\
\hline BGW012 & 5 & Congaree Monument & $33-49.56 \mathrm{~N}$ & $80-49.42 \mathrm{~W}$ \\
\hline BGW013 & 5 & Congaree Monument & $33-49.30 \mathrm{~N}$ & $80-49.49 \mathrm{~W}$ \\
\hline BGW014 & $\overline{2}$ & Santee State Park & $33-30.89 \mathrm{~N}$ & $80-29.68 \mathrm{~W}$ \\
\hline BGW015 & 4 & Santee State Pa $\mathrm{k}$ & $33-31.10 \mathrm{~N}$ & $80-29.53 \mathrm{~W}$ \\
\hline BGW016 & $\frac{7}{4}$ & Santee State Park & $33-31.02 \mathrm{~N}$ & $80-29.40 \mathrm{~W}$ \\
\hline BGW017 & 2 & Sancee State Park & $33-31.40 \mathrm{~N}$ & $80-29.63 \mathrm{~W}$ \\
\hline TGW018 & $T$ & Congaree Monument & $33-49.76 \mathrm{~N}$ & $80-49.17 \mathrm{~W}$ \\
\hline BGW019 & $\overline{4}$ & Francis Beidler For st & $33-13.31 \mathrm{~N}$ & $80-21.25 \mathrm{~W}$ \\
\hline BGW020 & 4 & Francis Beidler & $33-13.12 \mathrm{~N}$ & $80-21.25 \mathrm{~W}$ \\
\hline BGW021 & 2 & Jackson Audubon & $33-19.41 \mathrm{~N}$ & $81-50.15 \mathrm{~W}$ \\
\hline BGW022 & 5 & Jackson Audubon & $33-19.10 \mathrm{~N}$ & $81-52.13 \mathrm{~W}$ \\
\hline BGW023 & 3 & Jackson Audubon & $33-19.58 \mathrm{~N}$ & $81.51 .77 \mathrm{~W}$ \\
\hline BGW024 & 3 & Jackson Audubon & $33-21.13 \mathrm{~N}$ & $81-51.88 \mathrm{~W}$ \\
\hline BGW025 & $\overline{2}$ & Jackson Audubon & $33.19 .06 \mathrm{~N}$ & $81-51.01 \mathrm{~W}$ \\
\hline BGWIQS & 4 & Francis Beidler Forest & $33-13.12 \mathrm{~N}$ & $80-21.25 \mathrm{~W}$ \\
\hline BGW2OS & $T$ & Congaree Monument & $33-49.77 \mathrm{~N}$ & $80-49.21 \mathrm{~W}$ \\
\hline BGW3QS & 3 & Jackson Audubon & $33-21.13 \mathrm{~N}$ & $81-51.88 \mathrm{~W}$ \\
\hline
\end{tabular}

Table 6. Locations and Sample Numbers of Duplicate Soil Cores

\begin{tabular}{|c|c|c|}
\hline Location & Sample Number & Location Number \\
\hline \multirow{3}{*}{ Off-site } & BGW1QS & BGW020 \\
\cline { 2 - 3 } & BGW20S & BGW009 \\
\cline { 2 - 3 } & BGW30S & BGW024 \\
\hline \multirow{5}{*}{ On-site } & BGW40S & BGW058 \\
\cline { 2 - 3 } & BGW5QS & BGW066 \\
\cline { 2 - 3 } & BGW6OS & BGW036 \\
\cline { 2 - 3 } & BGW7QS & BGW069 \\
\cline { 2 - 3 } & BGW8OS & BGW074 \\
\hline
\end{tabular}


On-Site Sampling (August - September 1992)

The results for the on-site sarrpling are shown in Tables 7 and 8 which have the same contents as Tables 4 and 5 for the off-site sample sites. For the on-site samples, one duplicate (QS) core was collected for each soil group at the locations indicated in Table 6.

\section{Surveillances}

Two quality assurance surveillances were performed to verify that core collection and sampling procedures were performed in accordance with the approved Quality Assurance Project Plan for this task. Neither of these surveillances detected any deviations from the approved procedures.

\section{SUMMARY}

Eighty-three cores were collected at 75 sample stations during July, August, and September of 1992. Twenty-eight (28) of these cores were collected from off-site locations and fifty-five (55) were collected on the SRS. The method of sample collection was primarily vibracoring; however, at 16 sites, where the vibracore could not penetrate to deeper depths, a hand auger was used to collect the deeper samples.

A lithologic log was prepared for each soil core location. Each core was then divided into soil horizons and samples were collected from each horizon. These samples were packaged and given to SRS for shipping to a laboratory for analysis.

Core collection, sample collection, and sample packaging procedures were performed in accordance with the approved Quality Assurance Project Plan for this task. 
Table 7. Summary of Field Data for On-site Vibracores

\begin{tabular}{|c|c|c|c|c|c|c|}
\hline \multirow[b]{2}{*}{$\begin{array}{c}\text { Vibracore } \\
\text { Station }\end{array}$} & \multirow{2}{*}{$\begin{array}{c}\text { Date } \\
\text { Core } \\
\text { Collected }\end{array}$} & \multirow{2}{*}{$\begin{array}{c}\text { Date } \\
\text { Core } \\
\text { Sampled }\end{array}$} & \multirow{2}{*}{$\begin{array}{c}\text { Datie } \\
\text { Core } \\
\text { Shipped } \\
\end{array}$} & \multicolumn{3}{|c|}{ Field Results } \\
\hline & & & & $\begin{array}{c}\text { Core } \\
\text { Penetration } \\
\end{array}$ & $\begin{array}{c}\text { Core } \\
\text { Recovery }\end{array}$ & $\begin{array}{c}\text { Core } \\
\text { Compaction }\end{array}$ \\
\hline BGW026 & $8-24-92$ & $8-26-92$ & $8-26-92$ & $120^{\prime \prime}$ & $106.5^{\prime \prime}$ & $12^{\prime \prime}$ \\
\hline BGW027 & $9-08-92$ & $9-10-92$ & $9-11-92$ & $132^{\prime \prime}$ & $123.5^{\prime \prime}$ & $9^{\prime \prime}$ \\
\hline BGW028 & $8-24-92$ & $8-26-92$ & $8-28-92$ & $132^{\prime \prime}$ & $133^{\prime \prime}$ & $3^{\prime \prime}$ \\
\hline BGW029 & $8-31-92$ & $9-01-92$ & $5-03-92$ & $124^{\prime \prime}$ & $109^{\prime \prime}$ & 14" \\
\hline BGW030 & $8-20-92$ & $8-20-92$ & $8-24-92$ & $144^{\prime \prime}$ & $121^{\prime \prime}$ & $22^{\prime \prime}$ \\
\hline BGW031* & $8-20-92$ & $8-22-92$ & $8-24-92$ & $133^{\prime \prime}$ & $113^{\prime \prime}$ & $10^{\prime \prime}$ \\
\hline BGW032* & $8-18-92$ & $8-19-92$ & $8-20-92$ & $21^{\prime \prime}$ & $17^{\prime \prime}$ & $5^{\prime \prime}$ \\
\hline BGW033 & $8-12-92$ & $8-13-92$ & $8-13-92$ & $129^{\prime \prime}$ & $72^{\prime \prime}$ & $36^{\prime \prime}$ \\
\hline BGWn34 & $8-13-92$ & $8-13-92$ & $8-14-92$ & $138^{\prime \prime}$ & $91 "$ & $47^{\prime \prime}$ \\
\hline BGW035 & $8-12-92$ & $8-13-92$ & $8-14-92$ & $159^{\prime \prime}$ & $111^{11}$ & $41^{\prime \prime}$ \\
\hline BGW036* & $8-25-92$ & $8-26-92$ & $8-27-92$ & $120^{\prime \prime}$ & $120^{\prime \prime}$ & $8^{\prime \prime}$ \\
\hline BGW037* & $8-21-92$ & $8-22-92$ & $8-24-92$ & $140^{\prime \prime}$ & $90^{\prime \prime}$ & $48^{\prime \prime}$ \\
\hline BüV038 & $8-24-92$ & $8-26-92$ & $8-26-92$ & $115^{\prime \prime}$ & $102^{\prime \prime}$ & $15^{\prime \prime}$ \\
\hline BGW039* & $9-01-92$ & $9-02-92$ & $9-03-92$ & $43^{\prime \prime}$ & $35^{\prime \prime}$ & $5^{\prime \prime}$ \\
\hline BGWO10* & $8-19-92$ & $8-20-92$ & $8-21-92$ & $20^{\prime \prime}$ & $18 "$ & $2^{\prime \prime}$ \\
\hline BGW & $8-18.92$ & $8-19-92$ & $8-20-92$ & $32^{\prime \prime}$ & $20^{\prime \prime}$ &  \\
\hline BGW $042^{*}$ & $8-19.92$ & $8-20-92$ & $8-21-92$ & $44.5^{\prime \prime}$ & $39^{\prime \prime}$ & $4.5^{\prime \prime}$ \\
\hline BGW043* & $9.09-92$ & $9-10-92$ & 9.11 .92 & $70^{\prime \prime}$ & $63^{\prime \prime}$ & $6^{\prime \prime}$ \\
\hline BGW044* & $9-03.92$ & $9-04-92$ & $9-04-92$ & $42^{\prime \prime}$ & $38 "$ & $4^{\prime \prime}$ \\
\hline BGW045 & $8-25.92$ & $8-26-92$ & $8-27-92$ & $141^{\prime \prime}$ & $106^{\prime \prime}$ & $19^{\prime \prime}$ \\
\hline BGW046 & $8-25-92$ & $8-28-92$ & $8-31-92$ & $120^{\prime \prime}$ & $112^{\prime \prime}$ & $7^{\prime \prime}$ \\
\hline BGW047 & $9.02-92$ & $9-03-92$ & $9-04-92$ & $141^{\prime \prime}$ & $126^{\prime \prime}$ & $15^{\prime \prime}$ \\
\hline BGW048 & $8-20-92$ & $8-22-92$ & $8-24-92$ & $141^{\prime \prime}$ & $136^{\prime \prime}$ & $8^{\prime \prime}$ \\
\hline BGW049 & $8-31-92$ & $9-02-92$ & $9.03-92$ & $120^{\prime \prime}$ & $101^{\prime \prime}$ & $18^{\prime \prime}$ \\
\hline BGW050* & $8-31.92$ & $9-03-92$ & $9-04-92$ & $72^{\prime \prime}$ & $64^{\prime \prime}$ & $8 "$ \\
\hline BGW051 & $8-09-92$ & $8-10-92$ & $8-11-92$ & $122^{\prime \prime}$ & $101^{\prime \prime}$ & $22^{\prime \prime}$ \\
\hline BGW052 & $8-10-92$ & $8-12-92$ & $8-12-92$ & $133^{\prime \prime}$ & $120^{\prime \prime}$ & $13^{\prime \prime}$ \\
\hline BGW053 & $8-09.92$ & $8-10-92$ & $8-11-92$ & $144^{\prime \prime}$ & $129^{\prime \prime}$ & $13^{\prime \prime}$ \\
\hline BGW054 & $8-\frac{92}{92}$ & $8-12-92$ & $8-13-92$ & $142^{\prime \prime}$ & $125.5^{\prime \prime}$ & $14^{\prime \prime}$ \\
\hline BGWG55 & $80 y-92$ & $8-10-92$ & $8-11-92$ & $120^{\prime \prime}$ & $100^{\prime \prime}$ & $15^{\prime \prime}$ \\
\hline BGW056 & $8-09-92$ & $8-10-92$ & $8-11-92$ & $128^{\prime \prime}$ & $120^{\prime \prime}$ & $8^{\prime \prime}$ \\
\hline BGW057* & $8-11-92$ & $8-12-92$ & $8-13-92$ & $59^{\prime \prime}$ & $51^{\prime \prime}$ & $9^{\prime \prime}$ \\
\hline BGW058 & $8-10-92$ & $8-12-92$ & $8-12-92$ & $144^{\prime \prime}$ & $106^{\prime \prime}$ & $40^{\prime \prime}$ \\
\hline BGW059 & $8-09-92$ & $8-11-92$ & $8-12-92$ & $131^{\prime \prime}$ & $116^{\prime \prime}$ & $14^{\prime \prime}$ \\
\hline BGW060 & $8-09.92$ & $8-11-92$ & $8-11-92$ & $133^{\prime \prime}$ & $106^{\prime \prime}$ & $22^{\prime \prime}$ \\
\hline
\end{tabular}

(*) sites which required the use of a hand auger to collect sample below vibracore refusal (**) sites for which Core B was logged and sampled due to deeper penetration,more complete recovery, and less compaction in Core B 
Table 7. Summary of Field Data for On-site Vibracores (continued)

\begin{tabular}{|c|c|c|c|c|c|c|}
\hline \multirow[b]{2}{*}{$\begin{array}{c}\text { Vibracore } \\
\text { Station }\end{array}$} & \multirow[b]{2}{*}{$\begin{array}{c}\text { Date } \\
\text { Core } \\
\text { Collected } \\
\end{array}$} & \multirow[b]{2}{*}{  } & \multirow[b]{2}{*}{$\begin{array}{c}\text { Date } \\
\text { Core } \\
\text { Shipped }\end{array}$} & \multicolumn{3}{|c|}{ Field Results } \\
\hline & & & & $\begin{array}{c}\text { Core } \\
\text { Penetration } \\
\end{array}$ & $\begin{array}{c}\begin{array}{c}\text { Core } \\
\text { Recovery }\end{array} \\
\end{array}$ & $\begin{array}{c}\text { Core } \\
\text { Compaction } \\
\end{array}$ \\
\hline BGW061 & $8-17-92$ & $8-19-92$ & $8-19-92$ & $144^{\prime \prime}$ & $129.5^{\prime \prime}$ & $14^{\prime \prime}$ \\
\hline BGW062 & $8-17-92$ & $8-19-92$ & $8-19-92$ & 144" & $134^{\prime \prime}$ & $7^{\prime \prime}$ \\
\hline BGW063 & $8-17-92$ & $8-18-92$ & $8-19-92$ & $127^{\prime \prime}$ & $117^{11}$ & $9^{\prime \prime}$ \\
\hline BGW064 & $8-17-92$ & $8-19-92$ & $8-20-92$ & $148.5^{\prime \prime}$ & $143^{\prime \prime}$ & $6^{\prime \prime}$ \\
\hline BGW065* & $8-17-92$ & $8-18-92$ & 8.19 .92 & $40^{\prime \prime}$ & $36^{\prime \prime}$ & $2^{\prime \prime}$ \\
\hline BGW066 & $8-18-92$ & $8-20-92$ & $8-21-92$ & $139^{\prime \prime}$ & $126^{\prime \prime}$ & $10^{\prime \prime}$ \\
\hline BGW067 & $9-02-92$ & $9-04-92$ & $9-04-92$ & $120^{\prime \prime}$ & $101^{\prime \prime}$ & $19^{\prime \prime}$ \\
\hline BGW068 & $9-01-92$ & $9-02-92$ & $9-03-92$ & $149^{\prime \prime}$ & $116.5^{\prime \prime}$ & $21^{\prime \prime}$ \\
\hline BGW069 & $8-27-92$ & $8-29-92$ & $8-31.92$ & $132^{\prime \prime}$ & $109^{\prime \prime}$ & $20^{\prime \prime}$ \\
\hline BGW070 & $8-27-92$ & $8-30-92$ & $8-31-92$ & $150^{\prime \prime}$ & $99^{\prime \prime}$ & $36^{\prime \prime}$ \\
\hline BGW071 & $8.27-92$ & $8-28.92$ & $8-31-92$ & $146 "$ & $120^{\prime \prime}$ & $23^{\prime \prime}$ \\
\hline BGW072 & $8-27-92$ & $8-28-92$ & $8-28-92$ & $146^{\prime \prime}$ & $121^{\prime \prime}$ & $24^{\prime \prime}$ \\
\hline BGW073 & $8-27-92$ & $8-29.92$ & $8-31-92$ & $121^{\prime \prime}$ & $113^{\prime \prime}$ & $4^{\prime \prime}$ \\
\hline BGW074 & $9-08-92$ & $9.10-92$ & 9.11 .92 & $126^{\prime \prime}$ & $99 "$ & $22^{\prime \prime}$ \\
\hline BGW075* & $9-09-92$ & $9-10-92$ & $9-11-92$ & $120^{\prime \prime}$ & $101^{\prime \prime}$ & $20^{\prime \prime}$ \\
\hline BGW4QS & $8-10-92$ & $8-12-92$ & $8-13-92$ & $144^{\prime \prime}$ & $107^{11}$ & $32^{\prime \prime}$ \\
\hline BGW5OS & $8-18-92$ & $8-20-92$ & $8-20-92$ & $123^{\prime \prime}$ & $112^{\prime \prime}$ & $14^{\prime \prime}$ \\
\hline BGW60S" & $8-25-92$ & $8-26-92$ & $8-27-92$ & $120^{\prime \prime}$ & $120^{\prime \prime}$ & 0" \\
\hline BGW7QS & $8-28-92$ & $8-29-92$ & $8-31-92$ & $132^{\prime \prime}$ & $106^{\prime \prime}$ & $26^{\prime \prime}$ \\
\hline BGW8OS & $9-08-92$ & $9-10-92$ & $9-11-92$ & $130^{\prime \prime}$ & $96^{\prime \prime}$ & $32^{\prime \prime}$ \\
\hline
\end{tabular}

(*) sites which required the use of a hand auger to collect sample below vibracore refusal (**) sites for which Core B was logged and sampled due to deeper penetration, more complete recovery, and less compaction in Core B 
Table 8. Soil Groupings Encountered in On-site Vibracores

\begin{tabular}{|c|c|c|c|c|}
\hline \begin{tabular}{|c|} 
Vibracore \\
Station \\
\end{tabular} & $\begin{array}{c}\text { Soil } \\
\text { Group }\end{array}$ & Sample Location & $\begin{array}{c}\text { Latitude } \\
\text { (Degrees-Minutes) }\end{array}$ & $\begin{array}{c}\text { Longitude } \\
\text { (Degrees-Minutes) } \\
\end{array}$ \\
\hline BGW026 & 3 & Savannah River Site & $33-19.30 \mathrm{~N}$ & $81-41.41 \mathrm{~W}$ \\
\hline BGW027 & $\bar{T}$ & Savannah River Site & $33-18.48 \mathrm{~N}$ & $81-41.29 \mathrm{~W}$ \\
\hline BGW028 & $\overline{2}$ & Savannah River Site & $33-18.25 \mathrm{~N}$ & $81-41.56 \mathrm{~W}$ \\
\hline BGW029 & $\overline{4}$ & Savannah River Site & $33-11.54 \mathrm{~N}$ & $81-35.80 \mathrm{~W}$ \\
\hline BGW030 & 2 & Savannah River Site & $33-10.64 \mathrm{~N}$ & $81-36.50 \mathrm{~W}$ \\
\hline BGW031 & 3 & Savannah River Site & $33-10.76 \mathrm{~N}$ & $81-36.42 W$ \\
\hline BGW032 & 4 & Savannah River Site & $33-09.04 \mathrm{~N}$ & $81-35.21 \mathrm{~W}$ \\
\hline BGW033 & 2 & Savannah River Site & $33-24.16 \mathrm{~N}$ & $81-36.49 \mathrm{~W}$ \\
\hline BGW034 & 2 & Savannah River Site & $33-23.42 \mathrm{~N}$ & $81-36.74 W$ \\
\hline$\overline{B G W 035}$ & 1 & Savannah River Site & $33-24.16 \mathrm{~N}$ & $81-36.83 \mathrm{~W}$ \\
\hline BGW036 & 4 & Savannah River Site & $33-20.27 \mathrm{~N}$ & $81-40.64 \mathrm{~W}$ \\
\hline BGW037 & $\overline{2}$ & Savannah River Site & $33-22.54 \mathrm{~N}$ & $81-37.16 \mathrm{~W}$ \\
\hline BGW038 & 3 & Savannah River Site & $33-22.19 \mathrm{~N}$ & $81-36.96 \mathrm{~W}$ \\
\hline BGW039 & 4 & Savannah River Site & $33-21.57 \mathrm{~N}$ & $81-40.87 \mathrm{~W}$ \\
\hline BGW040 & 4 & Savannah River Site & $33-09.03 \mathrm{~N}$ & $81-35.94 \mathrm{~W}$ \\
\hline BGW041 & $\overline{4}$ & Savannah River Site & $33-09.52 \mathrm{~N}$ & $81-33.42 W$ \\
\hline BGW042 & 4 & Savannah River Site & $33-11.86 \mathrm{~N}$ & $81-32.44 W$ \\
\hline BGW043 & 4 & Savannah River Site & $33-18.42 \mathrm{~N}$ & $81-33.79 \mathrm{~W}$ \\
\hline BGW044 & 4 & Savannah River Site & $33-17.38 \mathrm{~N}$ & $81-28.93 \mathrm{~W}$ \\
\hline BGW045 & 1 & Savannah River Site & $33-22.21 \mathrm{~N}$ & $81-37.96 \mathrm{~W}$ \\
\hline BGW046 & $\overline{3}$ & Savannah River Site & $33-20.41 \mathrm{~N}$ & $81-36.52 \mathrm{~W}$ \\
\hline BGW047 & $\frac{\pi}{3}$ & Savannah River Site & $33-19.21 \mathrm{~N}$ & $81-35.44 \mathrm{~W}$ \\
\hline BGW048 & 2 & Savannah River Site & $33-20.01 \mathrm{~N}$ & $81-33.61 \mathrm{~W}$ \\
\hline BGW049 & 3 & Savannah River Site & $33-15.15 \mathrm{~N}$ & $81-36.81 \mathrm{~W}$ \\
\hline BGW050 & 2 & Savannah River Site & $33-14.64 \mathrm{~N}$ & $81-37.25 \mathrm{~W}$ \\
\hline BGW051 & 5 & Savannah River Site & $33-13.71 \mathrm{~N}$ & $81-47.27 \mathrm{~W}$ \\
\hline BGW052 & 5 & Savannah River Site & $33-14.13 \mathrm{~N}$ & $81-47.69 \mathrm{~W}$ \\
\hline BGW053 & 5 & Savannah River Site & $33-13.77 \mathrm{~N}$ & $81-47.44 \mathrm{~W}$ \\
\hline BGW054 & 5 & Savannah River Site & $33-14.02 \mathrm{~N}$ & $81-47.51 \mathrm{~W}$ \\
\hline BGW055 & 5 & Savannah River Site & $33.14 .30 \mathrm{~N}$ & $81-47.93 \mathrm{~W}$ \\
\hline BGW056 & 5 & Savannah River Site & $33-14.86 \mathrm{~N}$ & $81-48.28 \mathrm{~W}$ \\
\hline BGW057 & 5 & Savannah River Site & $33-13.83 \mathrm{~N}$ & $81-47.48 W$ \\
\hline BGW058 & 5 & Savannah River Site & $33-14.08 \mathrm{~N}$ & $81-47.57 \mathrm{~W}$ \\
\hline BGW059 & 5 & Savannah River Site & $33-14.17 \mathrm{~N}$ & $81-47.71 \mathrm{~W}$ \\
\hline BGW060 & 5 & Savannah River Site & $33-14.27 \mathrm{~N}$ & $81-47.82 W$ \\
\hline
\end{tabular}


Table 8. Soil Groupings Encountered in On-site Vibracores (continued)

\begin{tabular}{|l|c|l|c|c|}
\hline $\begin{array}{c}\text { Vibracore } \\
\text { Station }\end{array}$ & $\begin{array}{c}\text { Soil } \\
\text { Group }\end{array}$ & Sample Location & $\begin{array}{c}\text { Latitude } \\
\text { (Degrees-Minutes) }\end{array}$ & $\begin{array}{c}\text { Longitude } \\
\text { (Degrees-Minutes) }\end{array}$ \\
\hline BGW061 & 3 & Savannah River Site & $33-10.19 \mathrm{~N}$ & $81-34.91 \mathrm{~W}$ \\
\hline BGW062 & 3 & Savannah River Site & $33-10.73 \mathrm{~N}$ & $81-34.13 \mathrm{~W}$ \\
\hline BGW063 & 2 & Savannah River Site & $33-11.03 \mathrm{~N}$ & $81-29.39 \mathrm{~W}$ \\
\hline BGW064 & 3 & Savannah River Site & $33-12.57 \mathrm{~N}$ & $81-30.40 \mathrm{~W}$ \\
\hline BGW065 & 4 & Savannah River Site & $33-11.15 \mathrm{~N}$ & $81-32.03 \mathrm{~W}$ \\
\hline BGW066 & 3 & Savannah River Site & $33-13.57 \mathrm{~N}$ & $81-38.63 \mathrm{~W}$ \\
\hline BGW067 & 2 & Savannah River Site & $33-21.07 \mathrm{~N}$ & $81-37.96 \mathrm{~W}$ \\
\hline BGW068 & 1 & Savannah River Site & $33-21.05 \mathrm{~N}$ & $81-38.14 \mathrm{~W}$ \\
\hline BGW069 & 1 & Savannah River Site & $33-20.82 \mathrm{~N}$ & $81-38.68 \mathrm{~W}$ \\
\hline BGW070 & 1 & Savannah River Site & $33-19.99 \mathrm{~N}$ & $81-38.87 \mathrm{~W}$ \\
\hline BGW071 & 1 & Savannah River Site & $33-20.02 \mathrm{~N}$ & $81-38.82 \mathrm{~W}$ \\
\hline BGW072 & 1 & Savannah River Site & $33-20.10 \mathrm{~N}$ & $81-38.86 \mathrm{~W}$ \\
\hline BGW073 & 1 & Savannah River Site & $33-20.61 \mathrm{~N}$ & $81-38.68 \mathrm{~W}$ \\
\hline BGW074 & 2 & Savannah River Site & $33-19.49 \mathrm{~N}$ & $81-38.70 \mathrm{~W}$ \\
\hline BGW075 & 1 & Savannah River Site & $33-19.37 \mathrm{~N}$ & $81-39.34 \mathrm{~W}$ \\
\hline BGW40S & 5 & Savannah River Site & $33-14.08 \mathrm{~N}$ & $81-47.57 \mathrm{~W}$ \\
\hline BGW50S & 3 & Savannah River Site & $33-13.57 \mathrm{~N}$ & $81-38.63 \mathrm{~W}$ \\
\hline BGW60S & 4 & Savannah River Site & $33-20.27 \mathrm{~N}$ & $81-40.64 \mathrm{~W}$ \\
\hline BGW70S & 1 & Savannah River Site & $33-20.82 \mathrm{~N}$ & $81-38.68 \mathrm{~W}$ \\
\hline BGW80S & 2 & Savannah River Site & $33-19.59 \mathrm{~N}$ & $81-38.76 \mathrm{~W}$ \\
\hline
\end{tabular}


Appendix A

Core Logs 


\section{Appendix A - Core Logs}

The paragraphs below provide a guide to the information contained in Appendix A. The first paragraph describes the calculation of sample intervals for cores where compaction occurred. The second paragraph defines the terms (eg. compaction, recovery) used in the core logs.

\section{Calculations}

Calculations were performed for each core to correct for compaction, which was averaged over th: entire length of the core. The following example calculation was prepared for core BGW 008 .

Core BGW 008 (A) compacted 6.5 inches, or 5 percent $(6.5 / 132=5 \%)$. Each sample interval was assumed to compact to 95 percent of its original length. Accordingly, each sample interval was multiplied by 0.95 , which divides the compaction proportionally over each sample interval. The calculations for core BGW 008 were as follows:

Interval (in)

$0-20$

$20-40$

$40-60$

$60-90$

$90-120$
Compaction Factor

0.95

0.95

0.95

0.95

0.95
Corrected Interval (in)

$\begin{array}{ll}= & 0-19 \\ = & 19-38 \\ = & 38-57 \\ = & 57-86 \\ = & 86-114\end{array}$

\section{Definitions}

The following terms appear on the eighty-three core logs contained in Appendix A.

CLIENT - This task was completed by Metcalf \& Eddy, Inc. and Athena Technologies, Inc. for Westinghouse Savannah River Company

TIME - Time core was collected

\section{PROJECT - Background Wetlands Soils Study}

CORE DATE - Date core was collected

CORE DEPTH - Total inches of penetration by the aluminum vibracoring sample tube

COMPACTION - Compaction (inches and percent) of the soil core within the aluminum tube

RECOVERY - Length of soil core retrieved (core depth minus compaction and lcis of material from bottom of core). For cores collected by vibracore and hand auger, recovery for both methods is provided (eg. BGW 003 - RECOVERY - 67"/120" (vibracore/auger)) 
LOG DATE - Date core was opened, sampled, and described (lithologic log) by geologist

CORE COORDINATES - Latitude and longitude of core sampling location as measured with Global Positioning System (GPS)

LOGGER - Initials of geologist that prepared lithologic description

\section{RSK Richard S. Keenan \\ WJS Walter J. Sexion}

CORE \# - Predesignated core location number

SAMPLE INTERVAL - Sample depths in inches for Core A and Core B for sample intervals A through $E$, accounting for compaction. For cores collected by hand auger, only one core (A) was collected, with no compaction.

SOIL PROFILE - Graphical representation of soil core that was logged and sampled (Core A or Core B). Depth of graphical description may exceed depth of deepest sampling interval.

DESCRIPIION - Lithologic description of soil core that was sub-sampled for chemical analysis.

COLLECTED BY - Initials of field crew present when samples were collected
WJS Walter J. Sexton
MAB Michael A. Bise
GWS Glenn W. Christiansen
RSK Richard S. Keenan
VR Vergil Rogers
PS Philip Sexton
KD Kenneth Dixon

WEATHER CONDIIIONS - Qualitative description of weather when samples were collected LOCATION - General description on sampling location (eg. Aiken State Park)

SOIL GROUP - Soil group 1-5 (Table 1) 







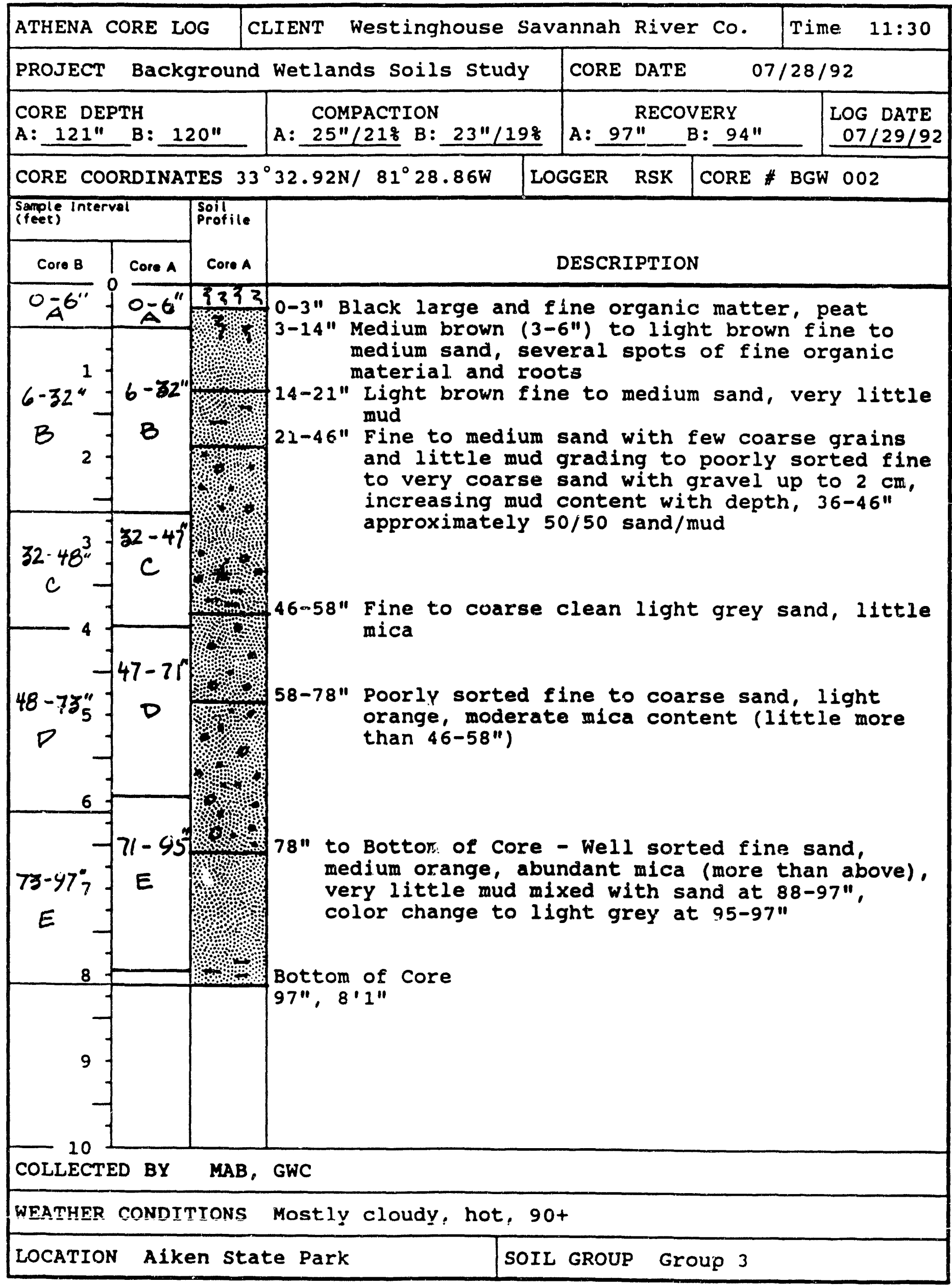














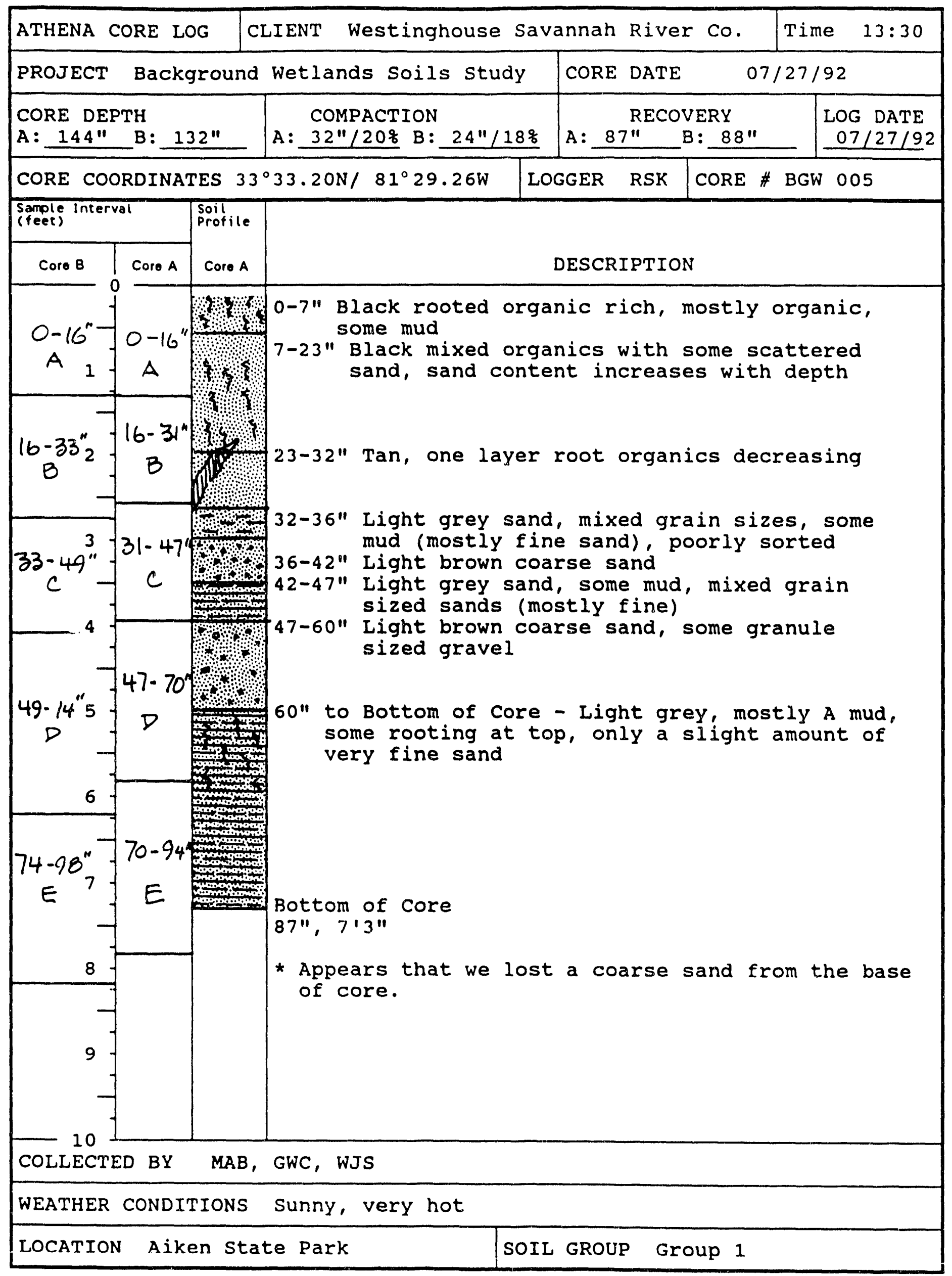





























































































































































































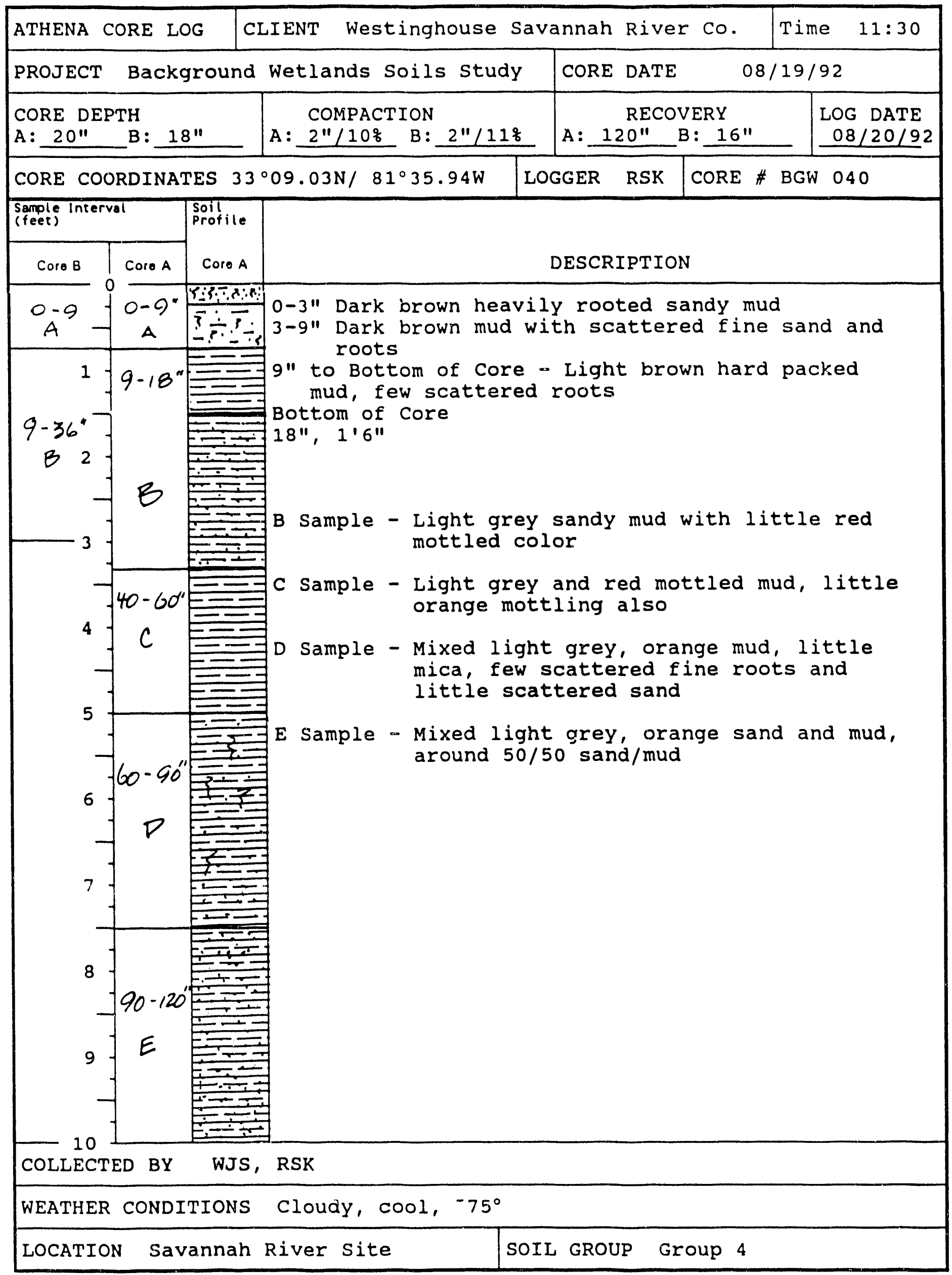









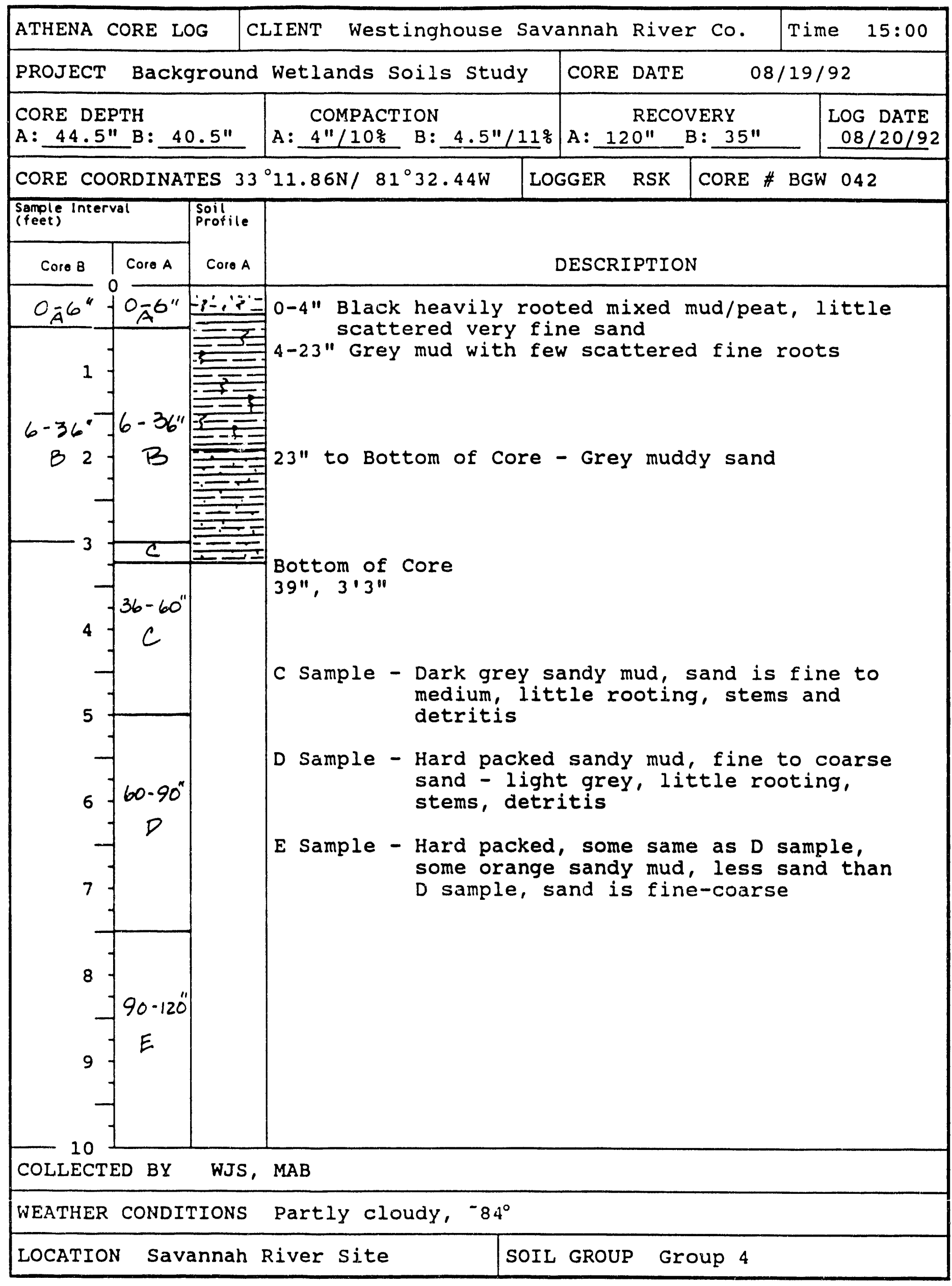




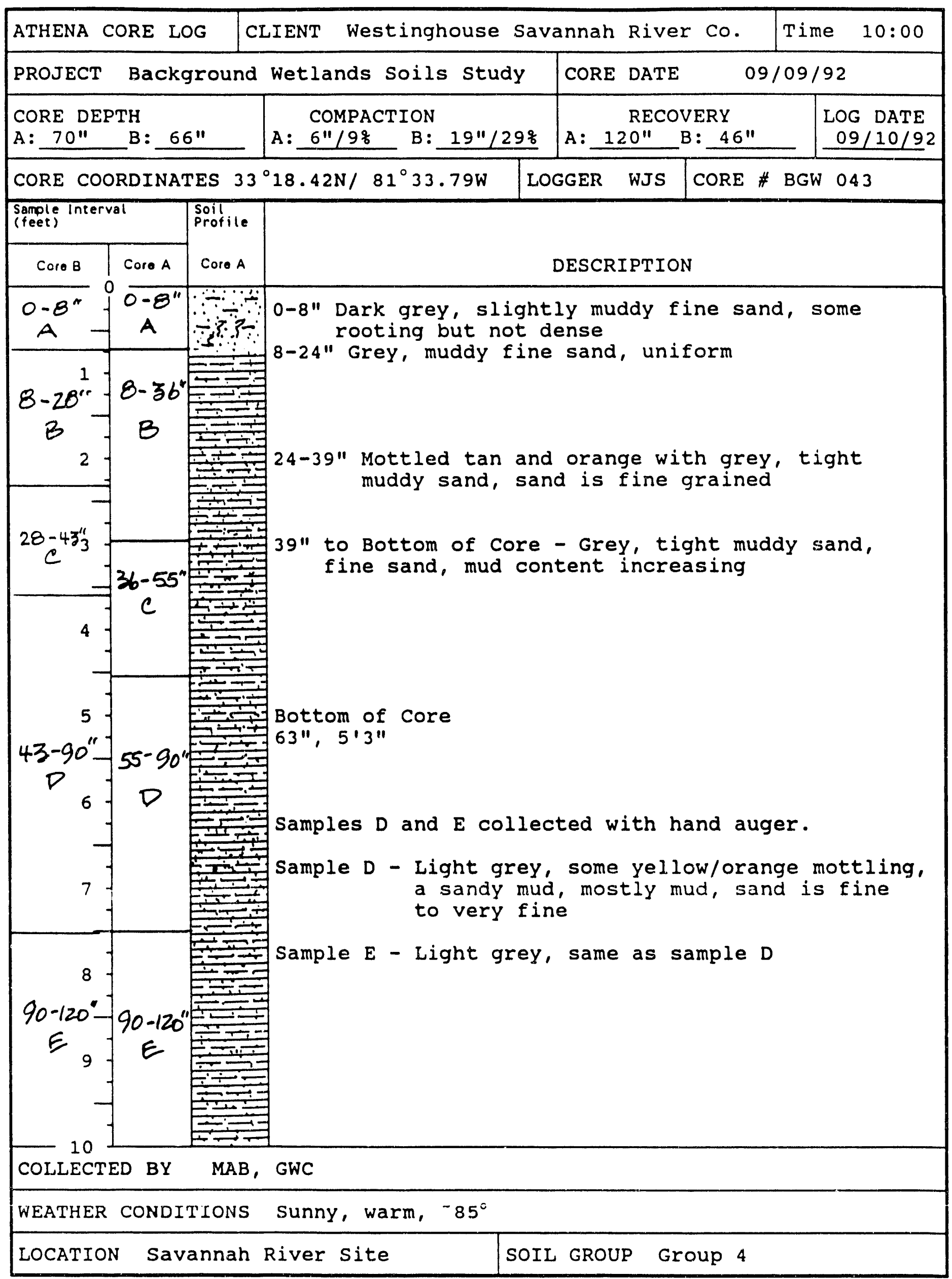




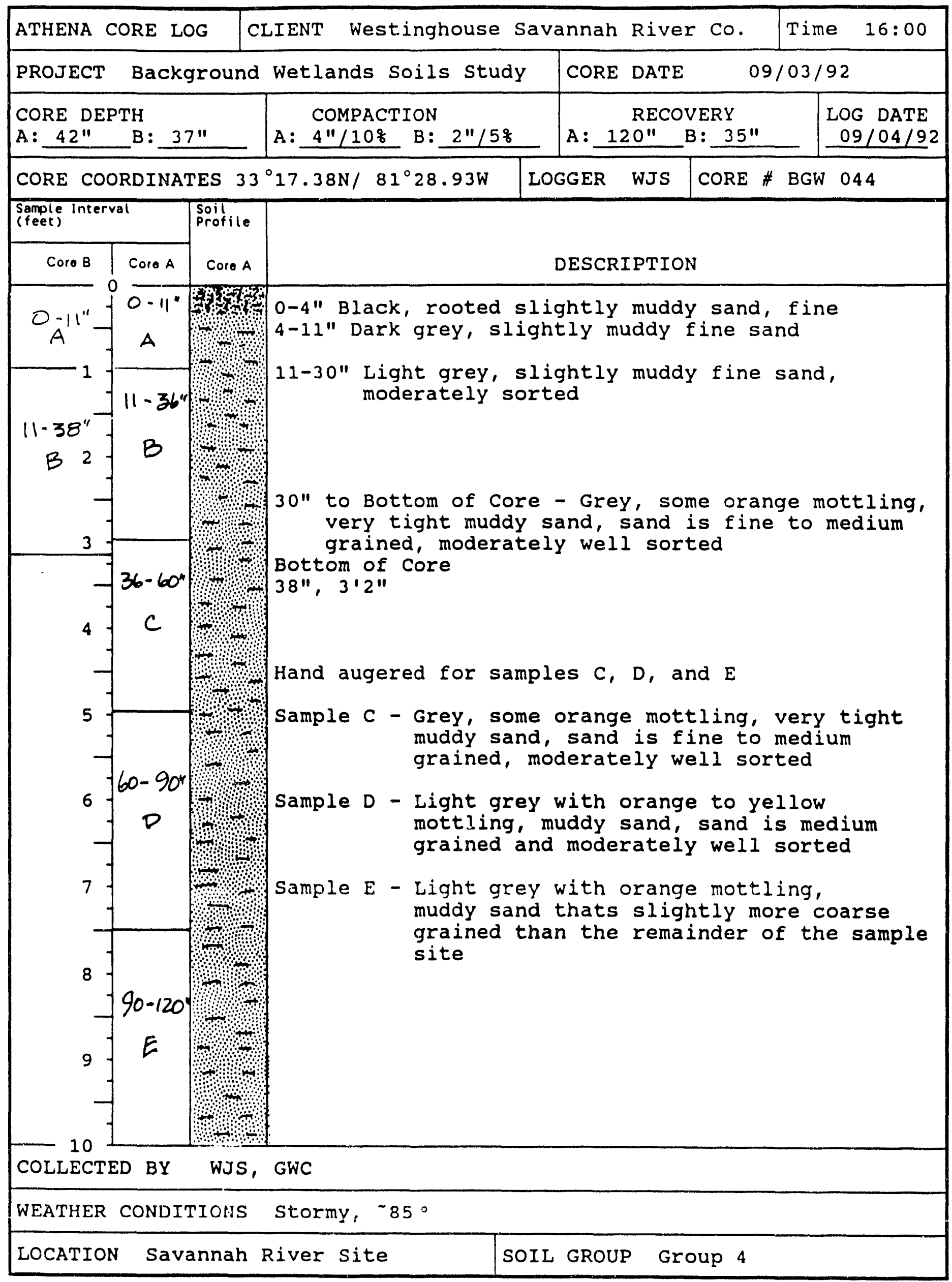




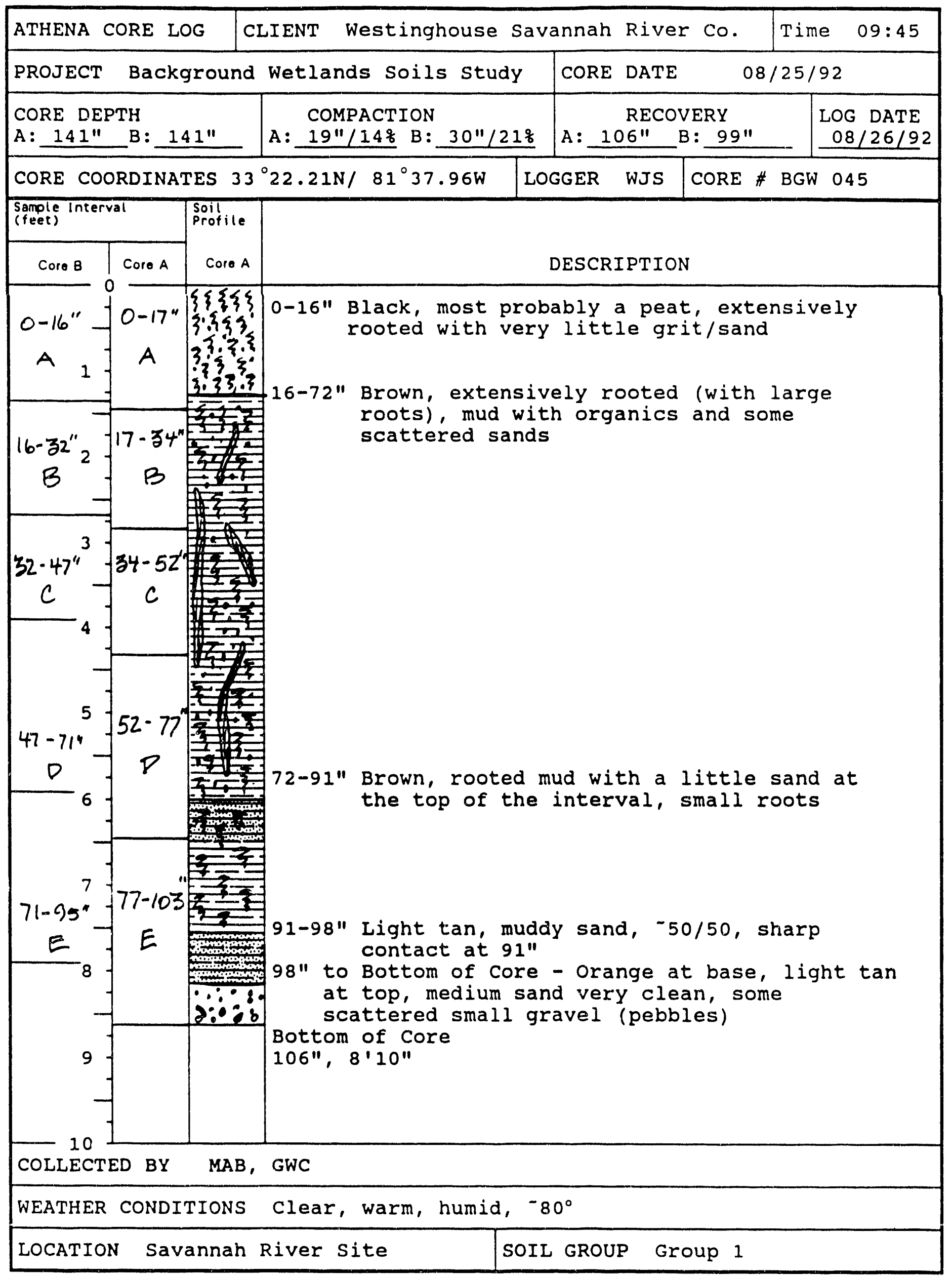




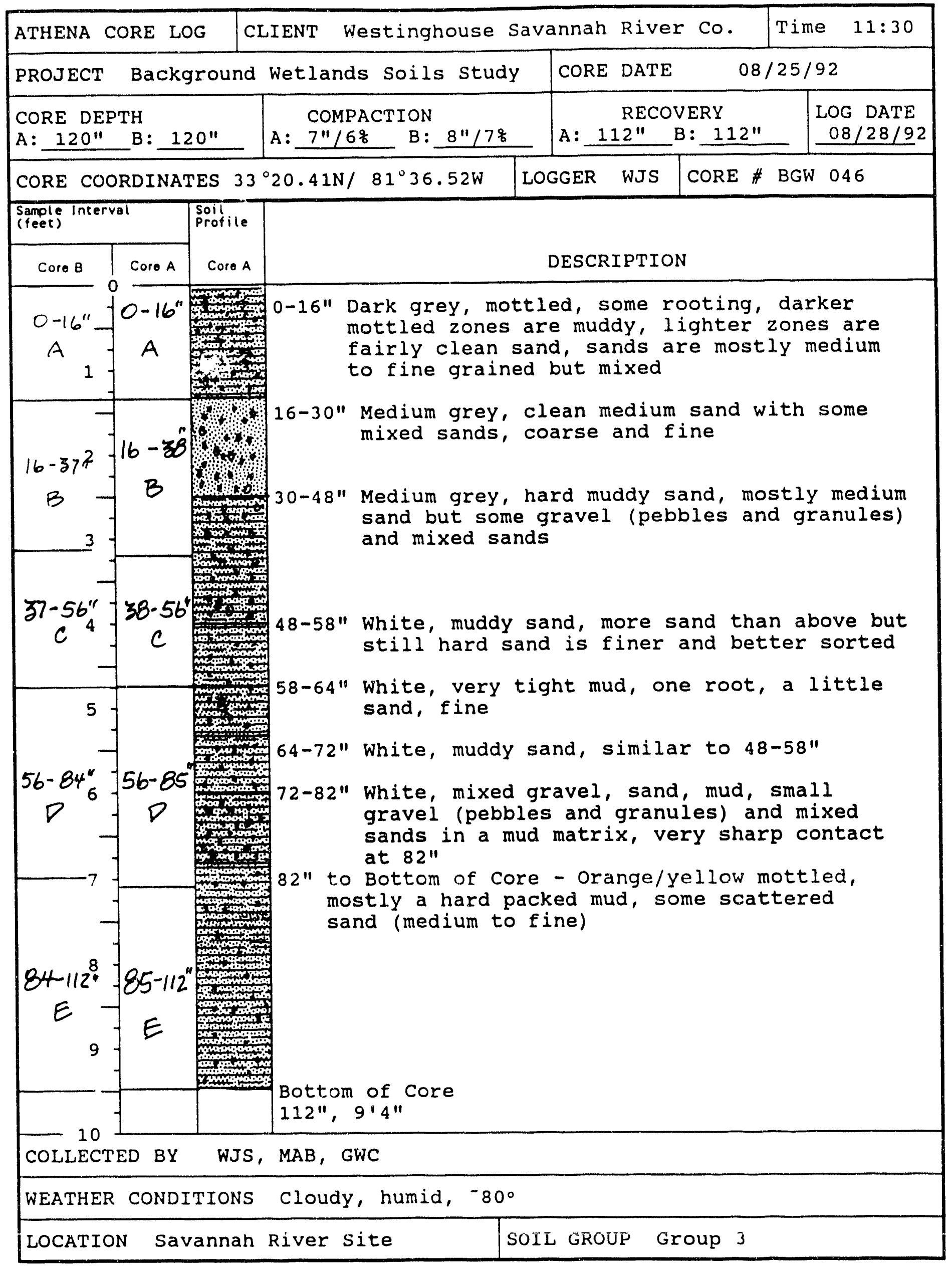




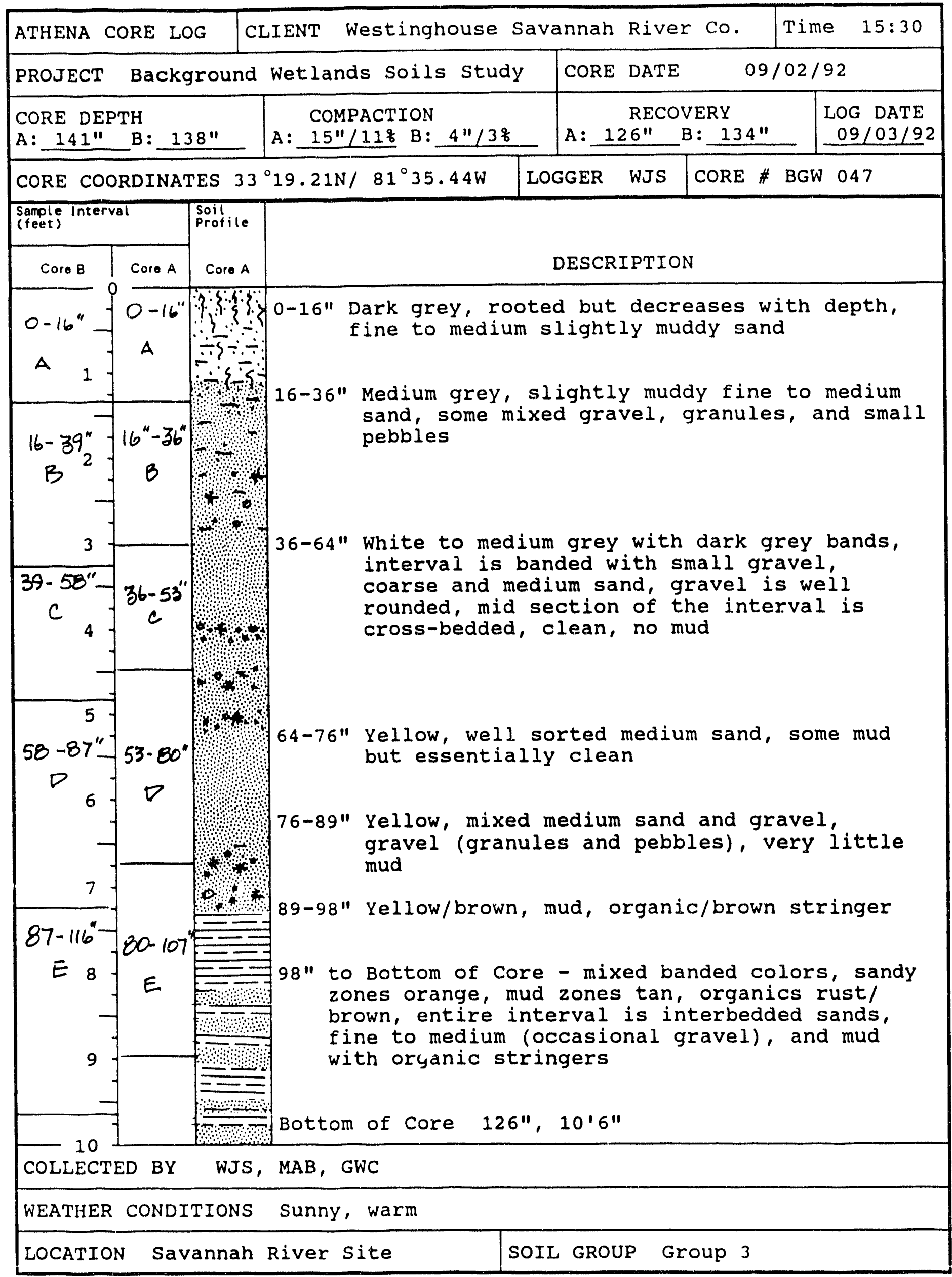




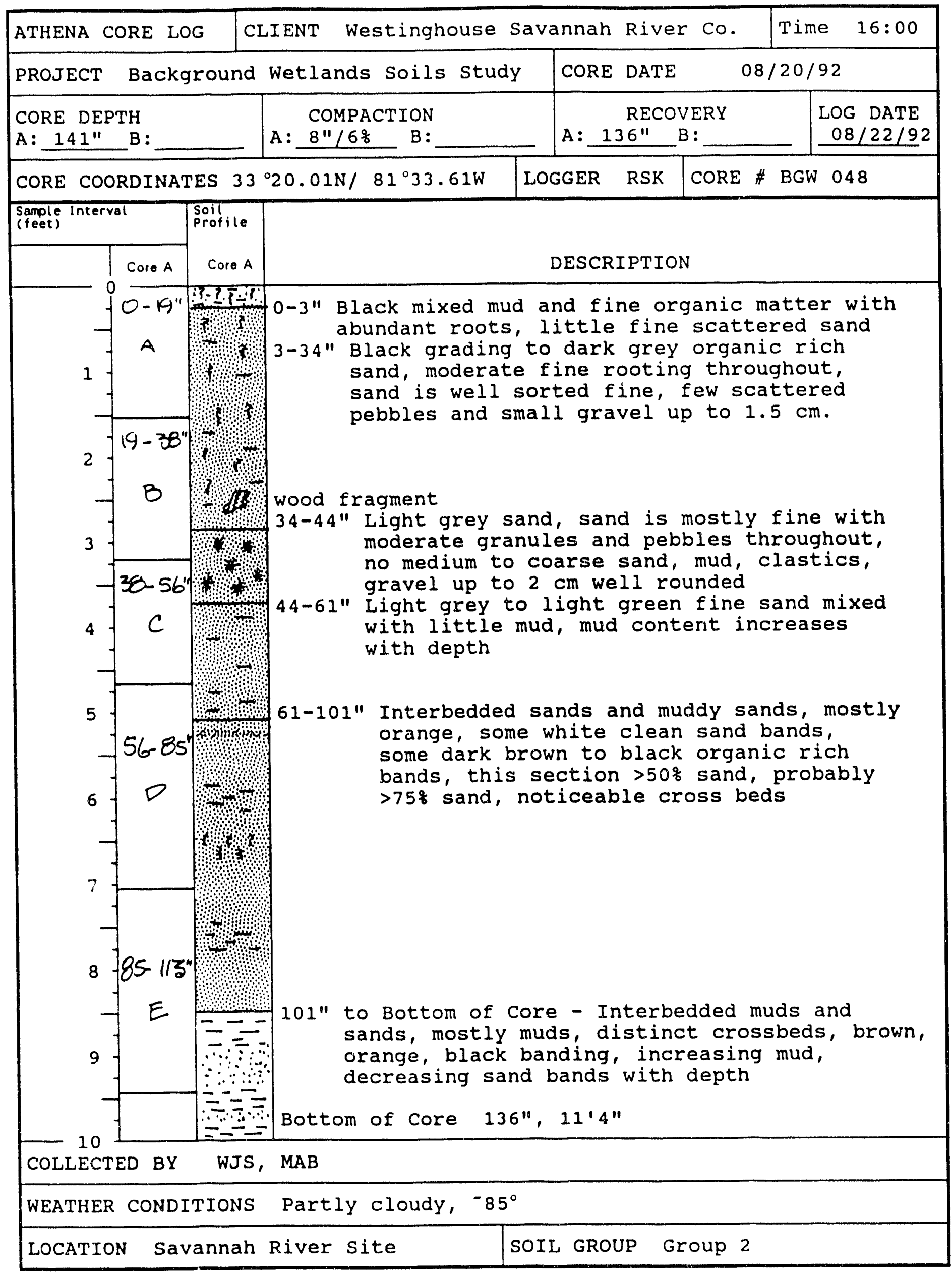




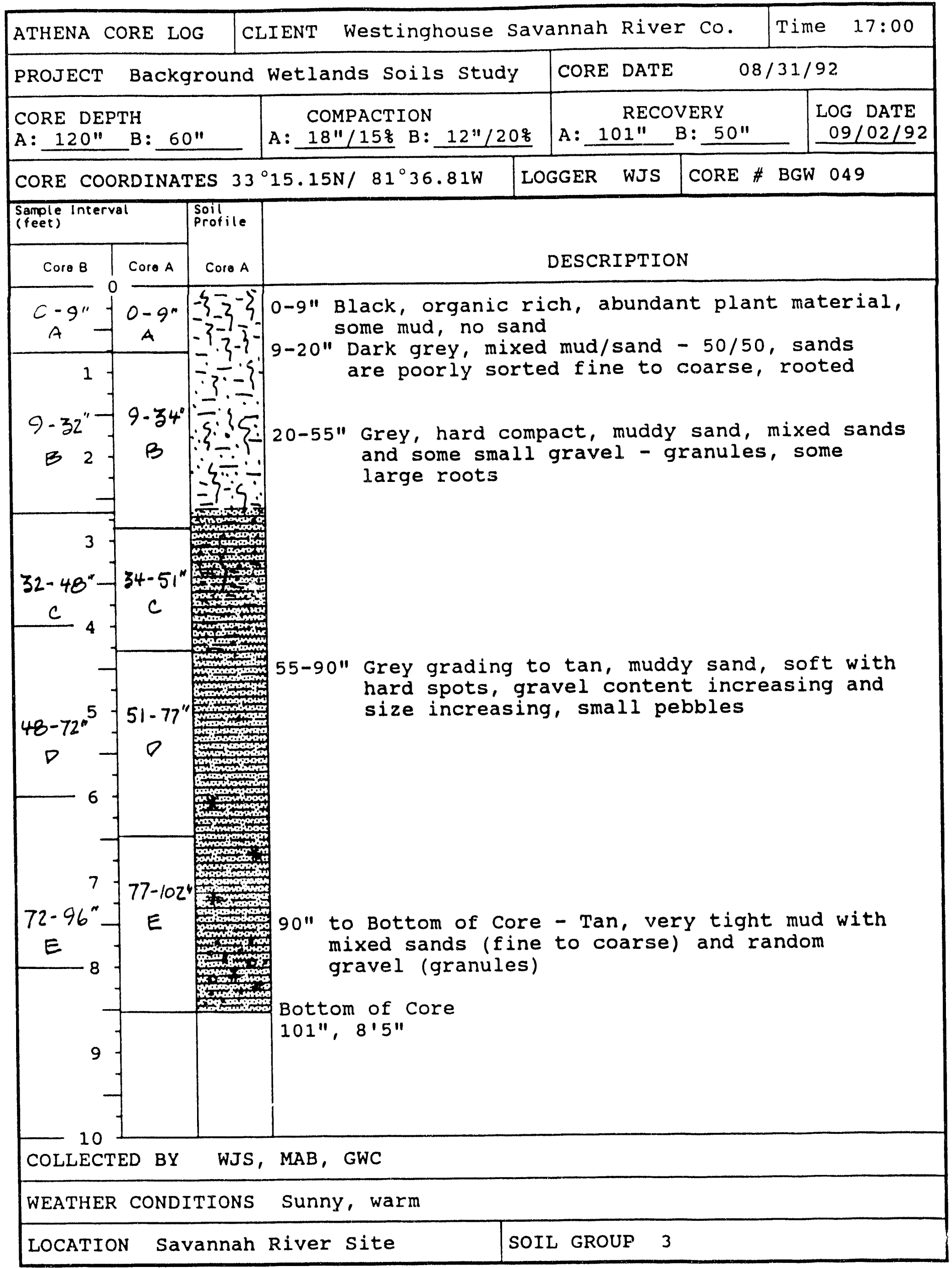




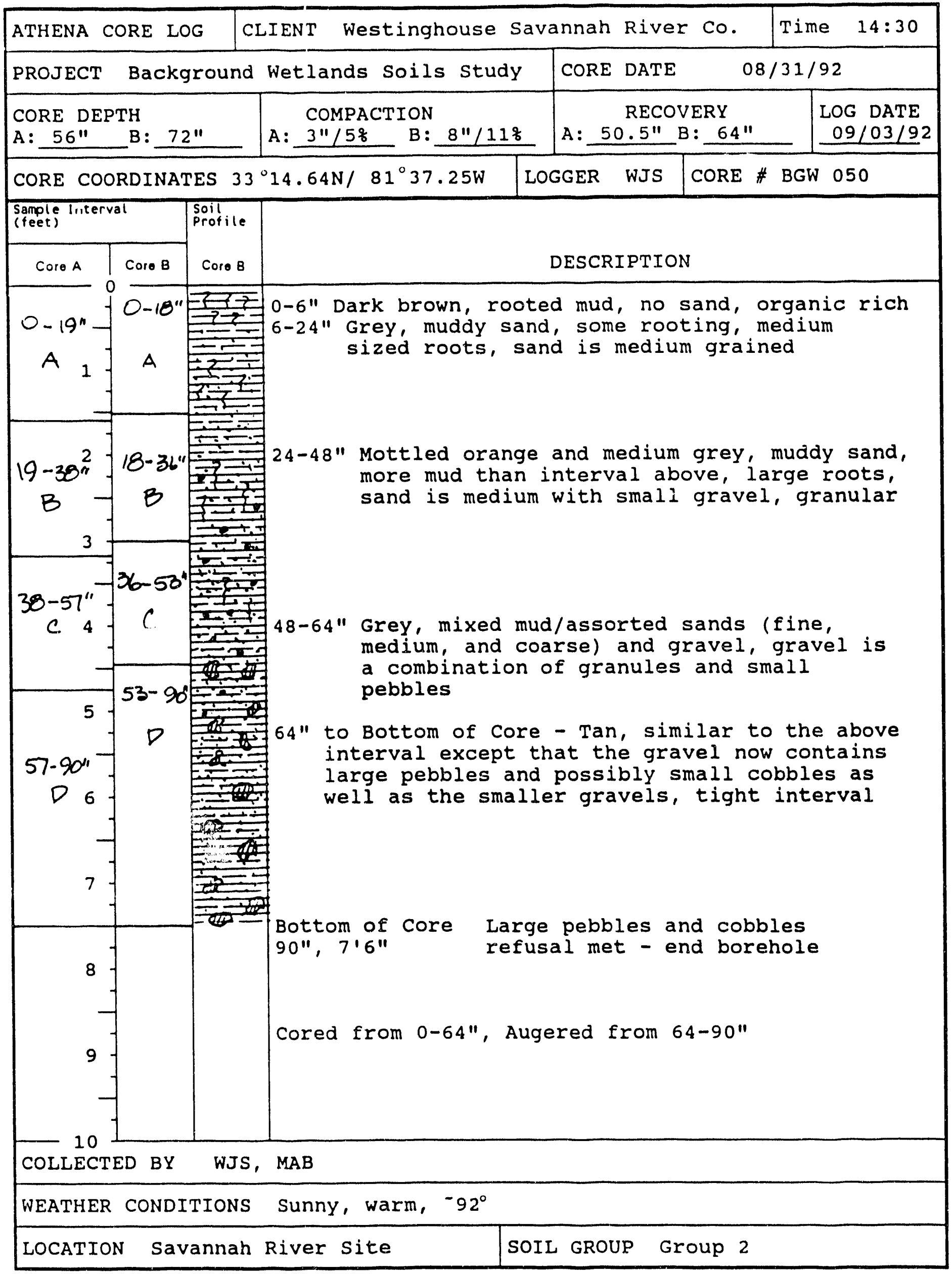
























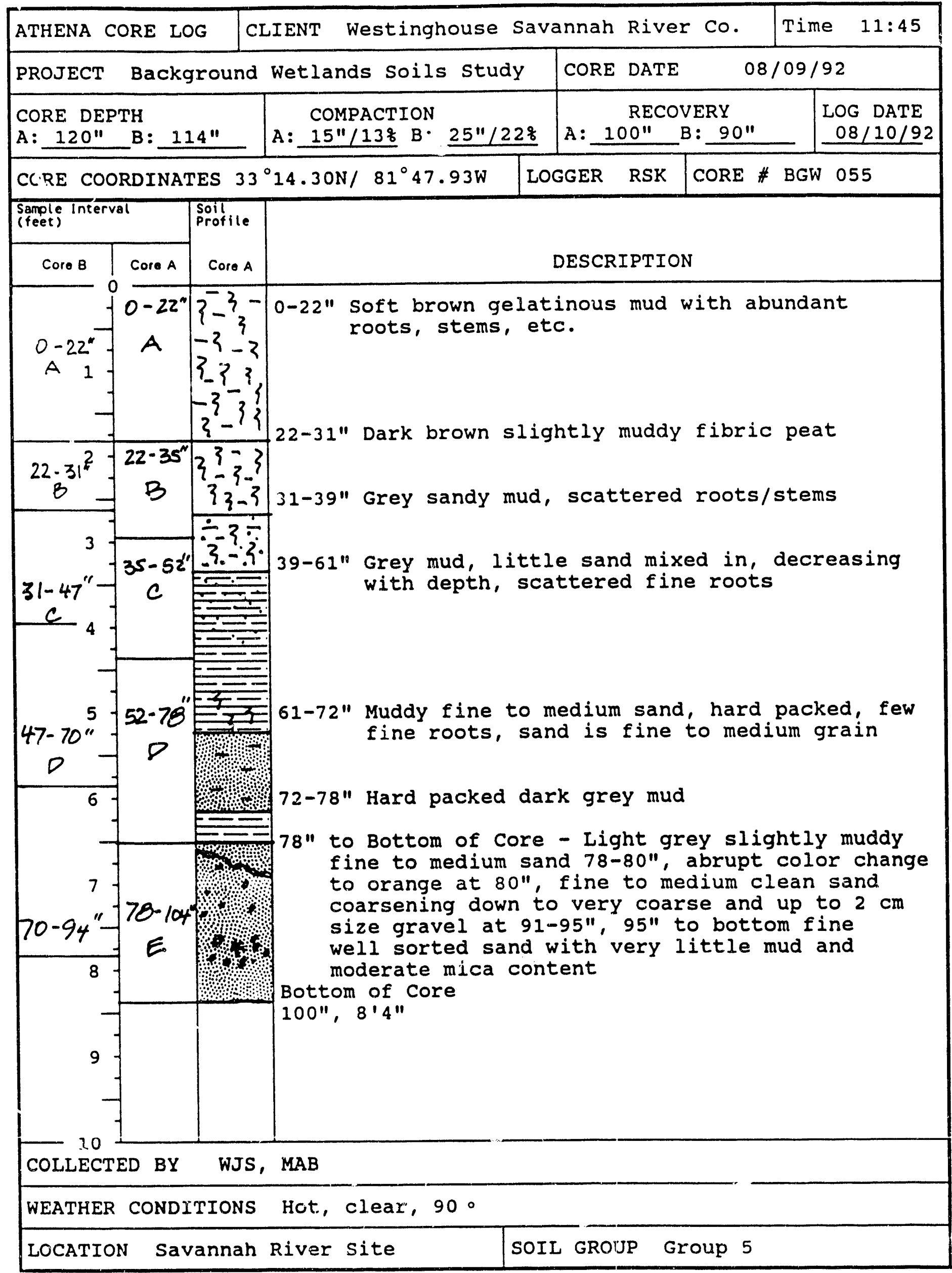









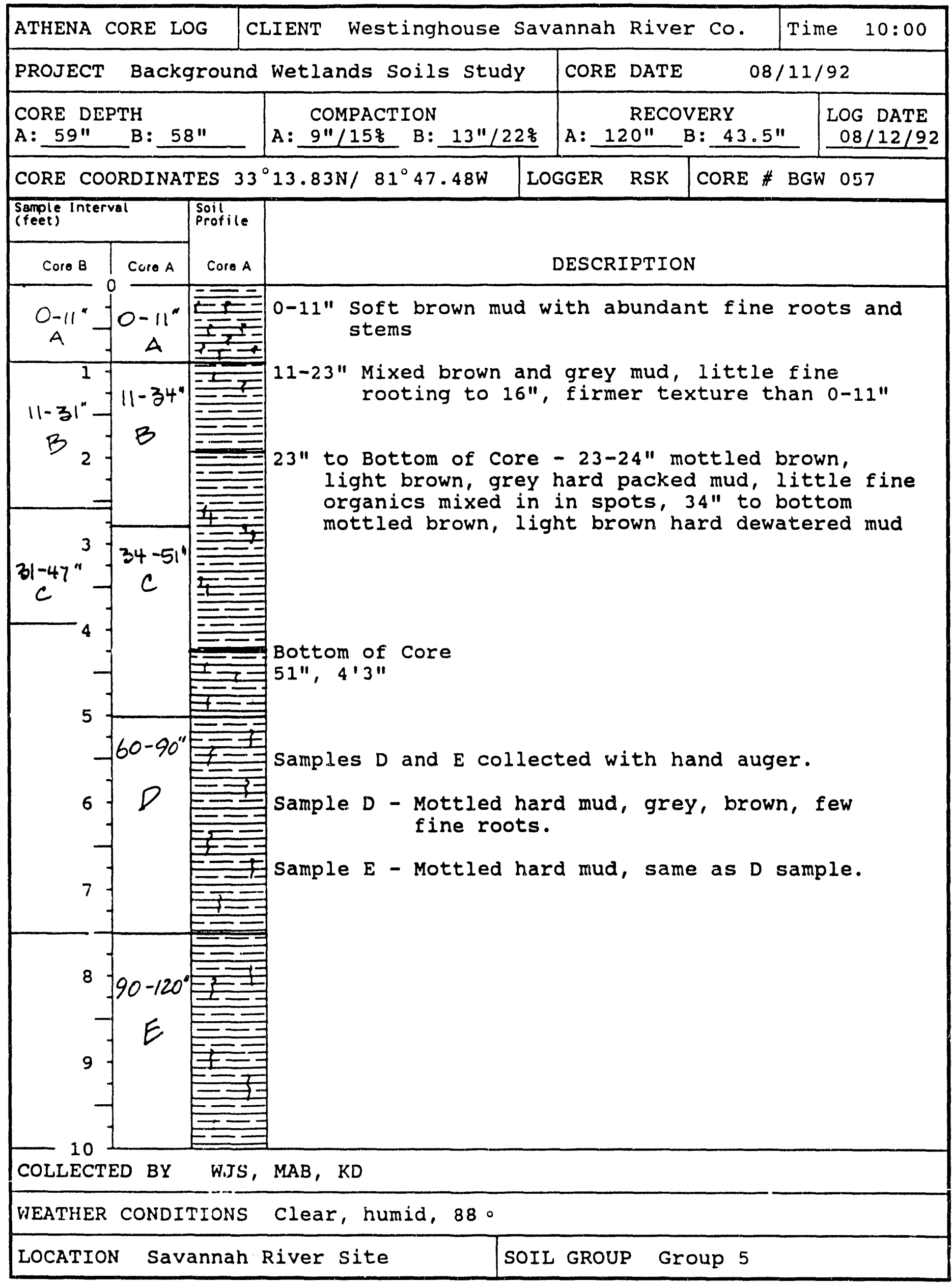




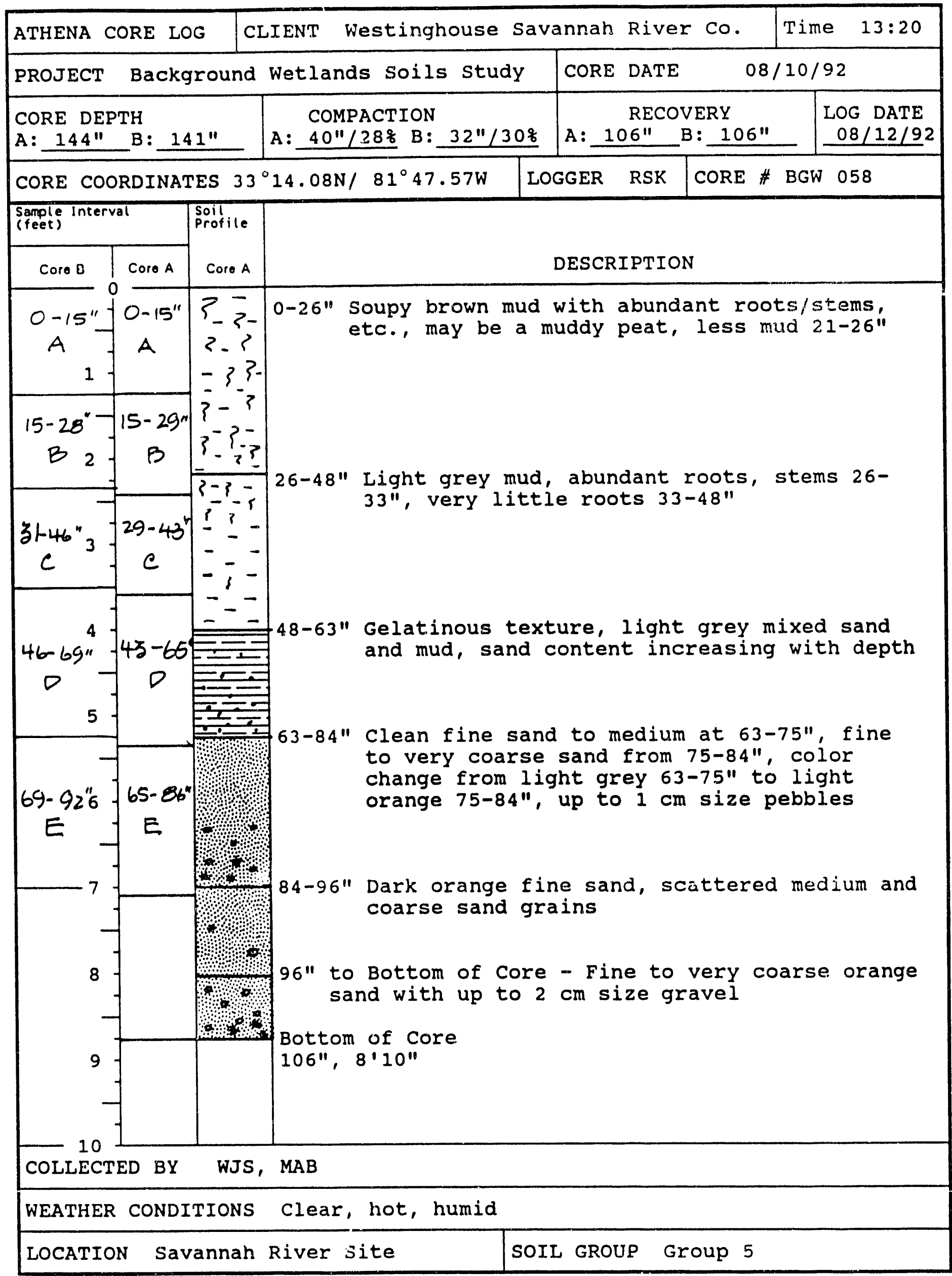






















































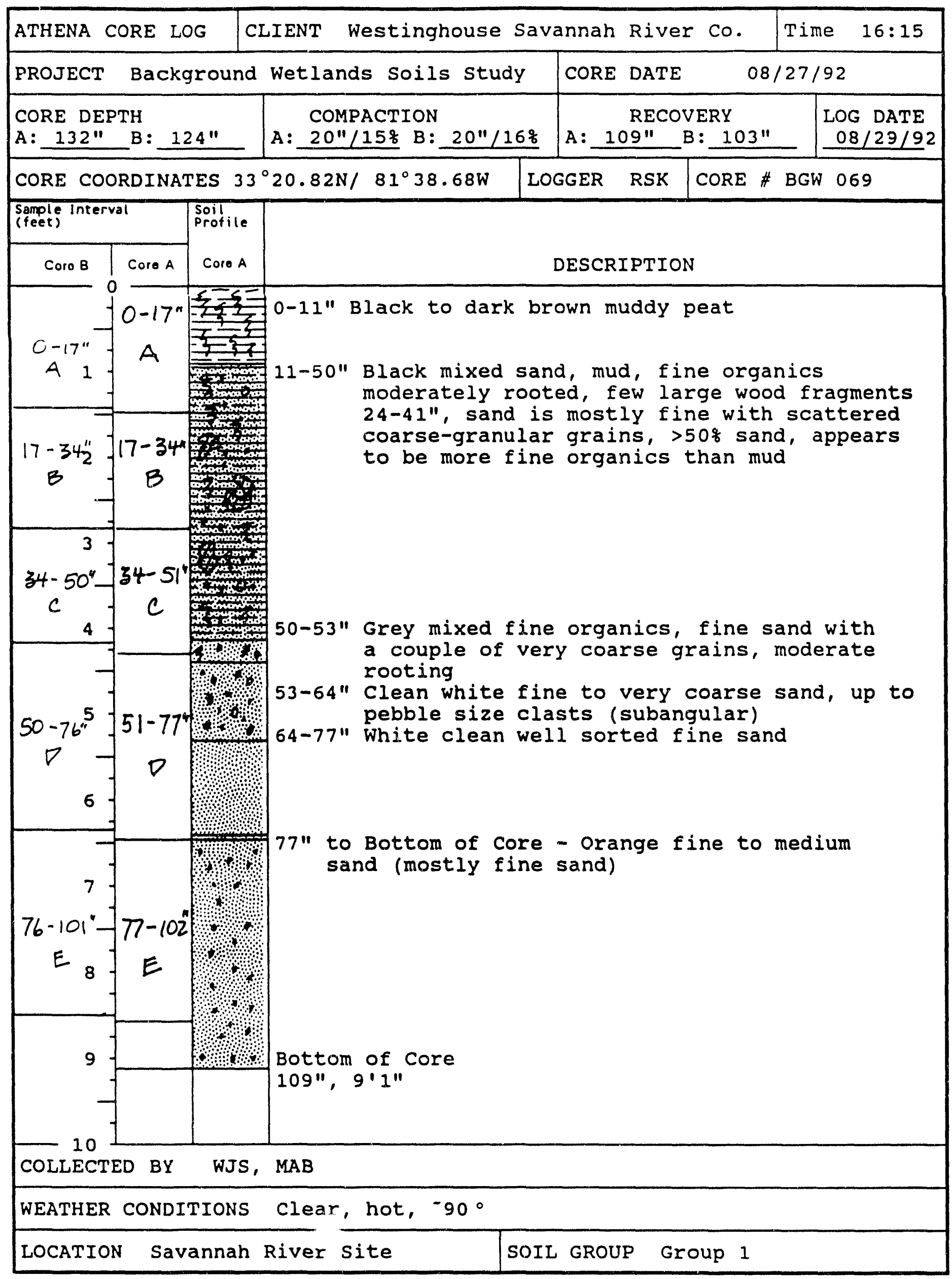



















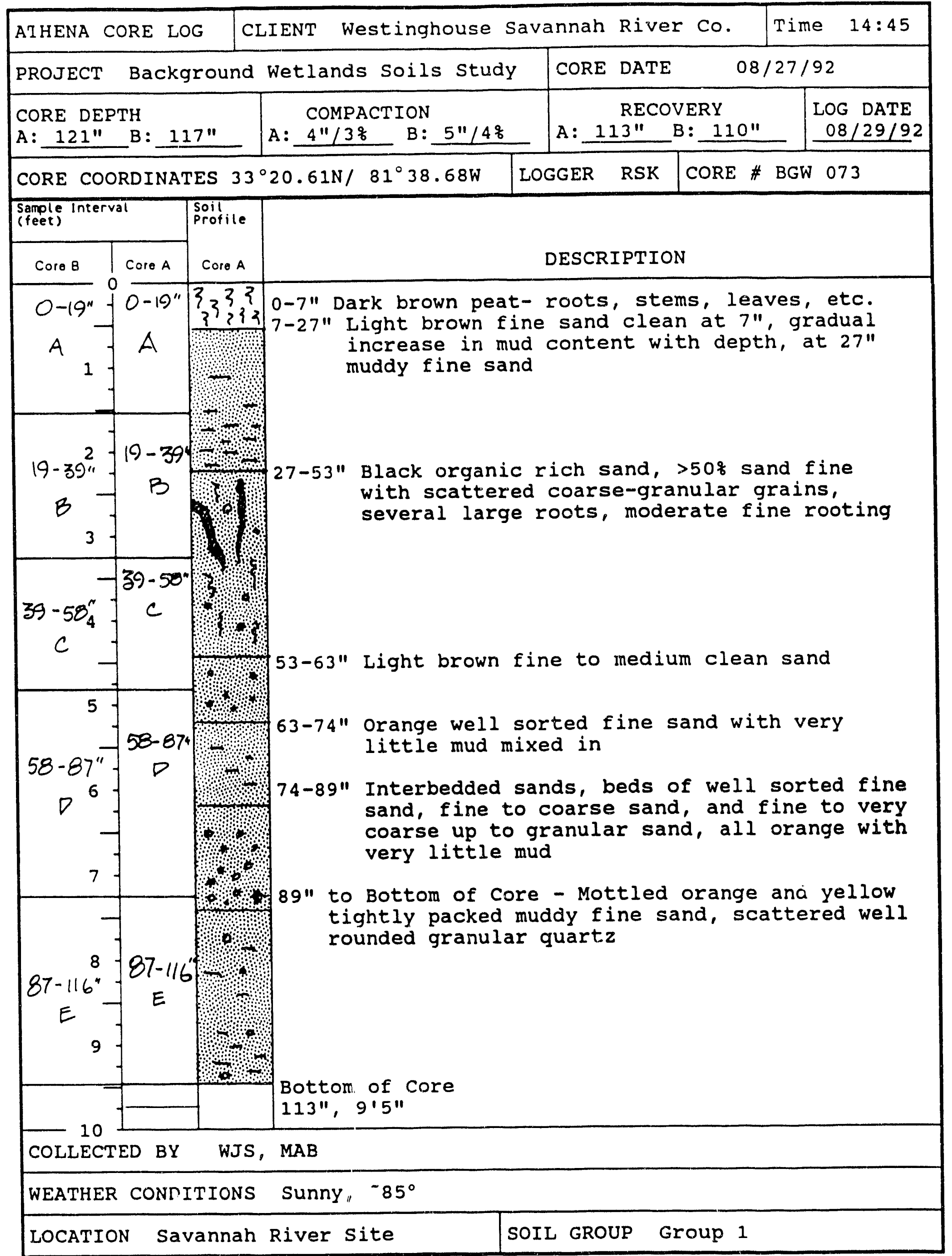




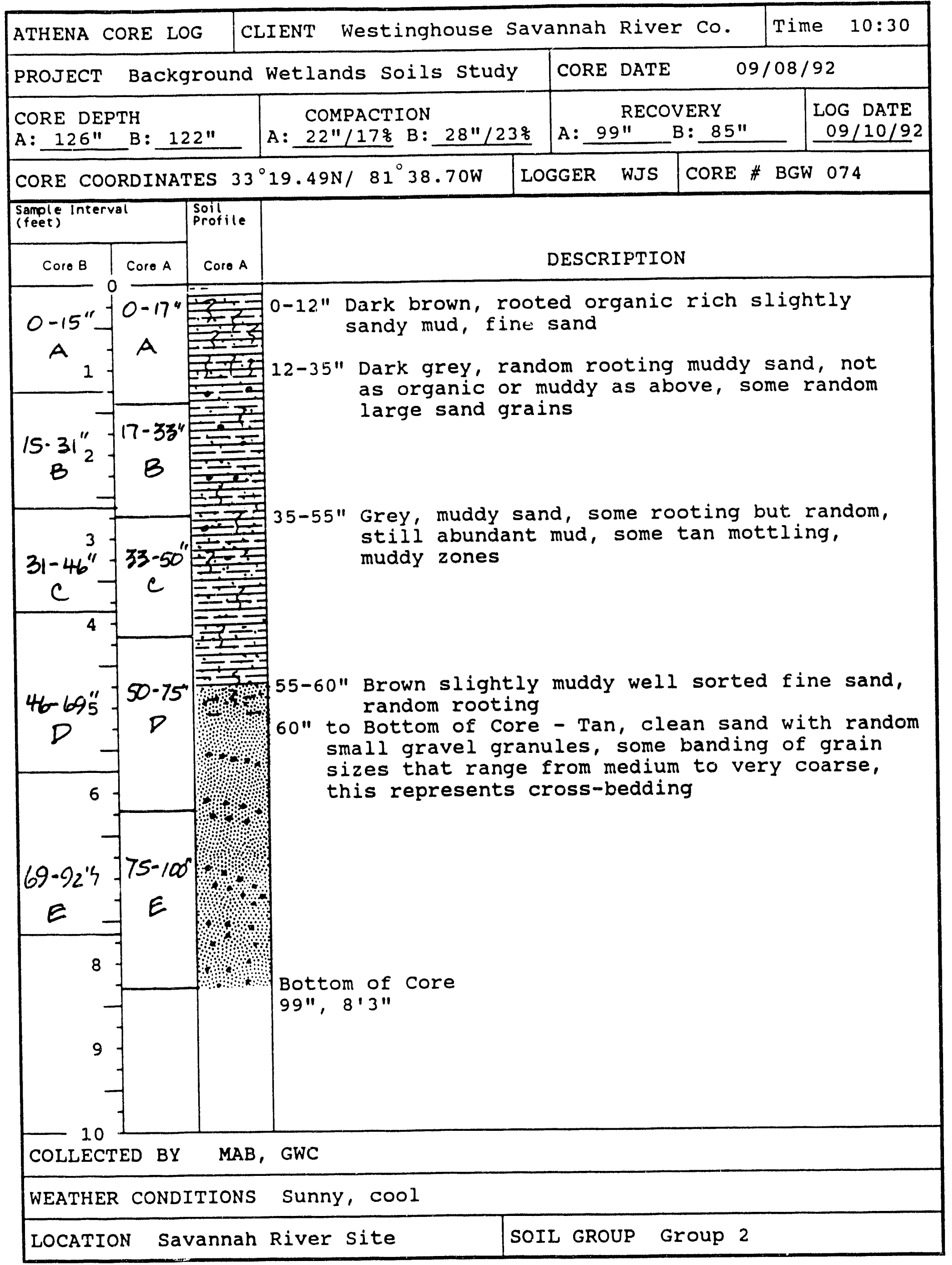




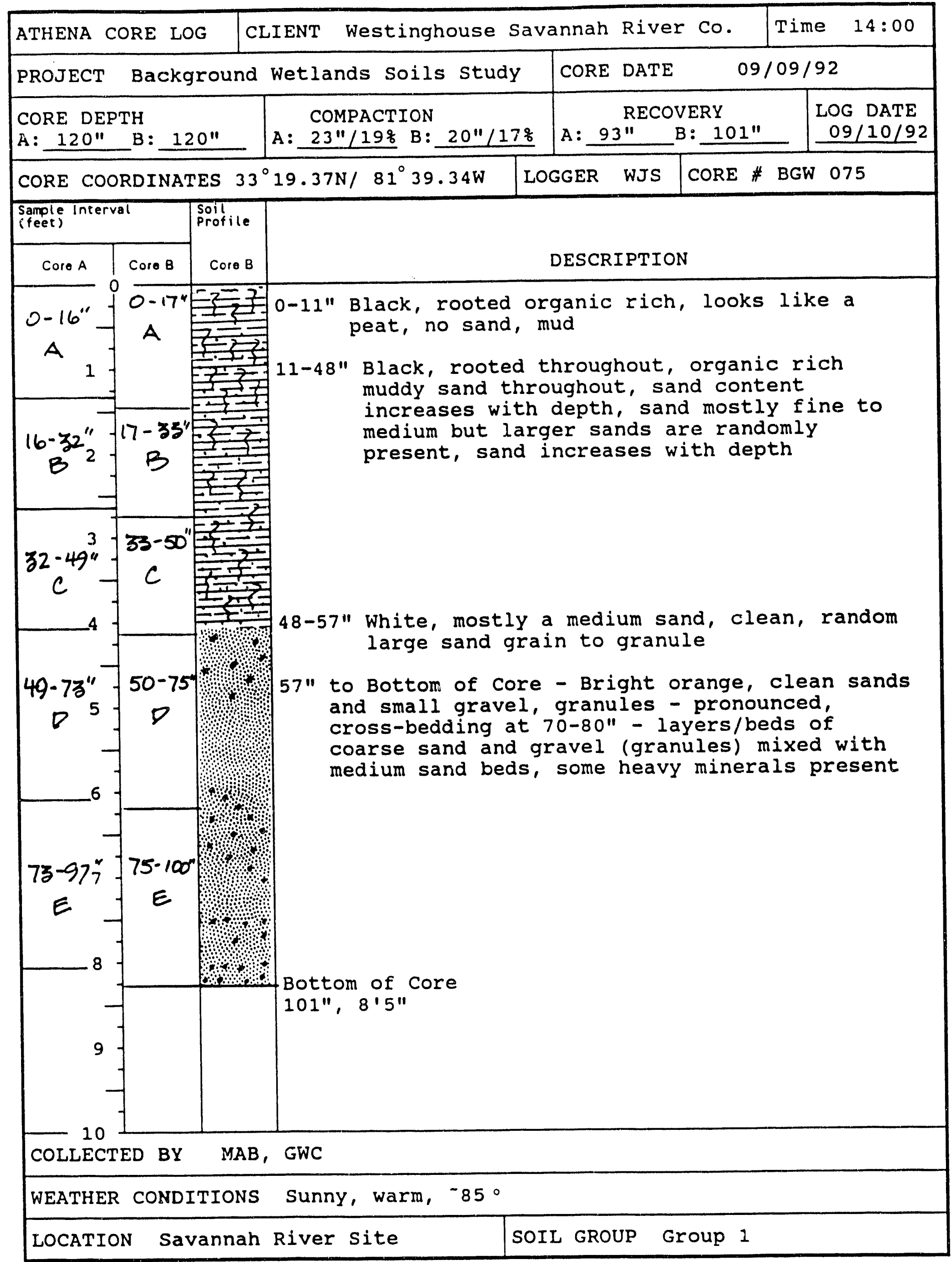









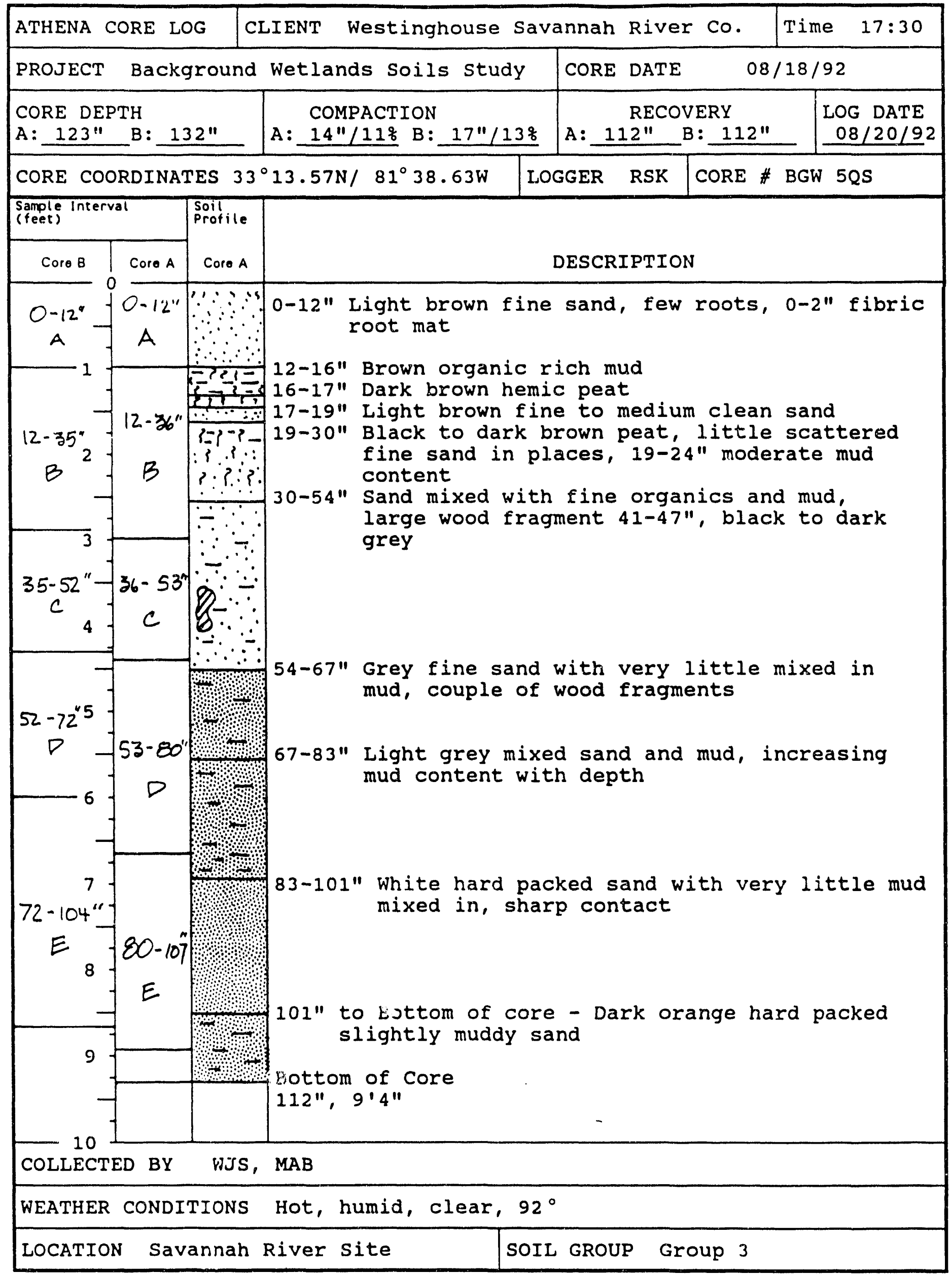




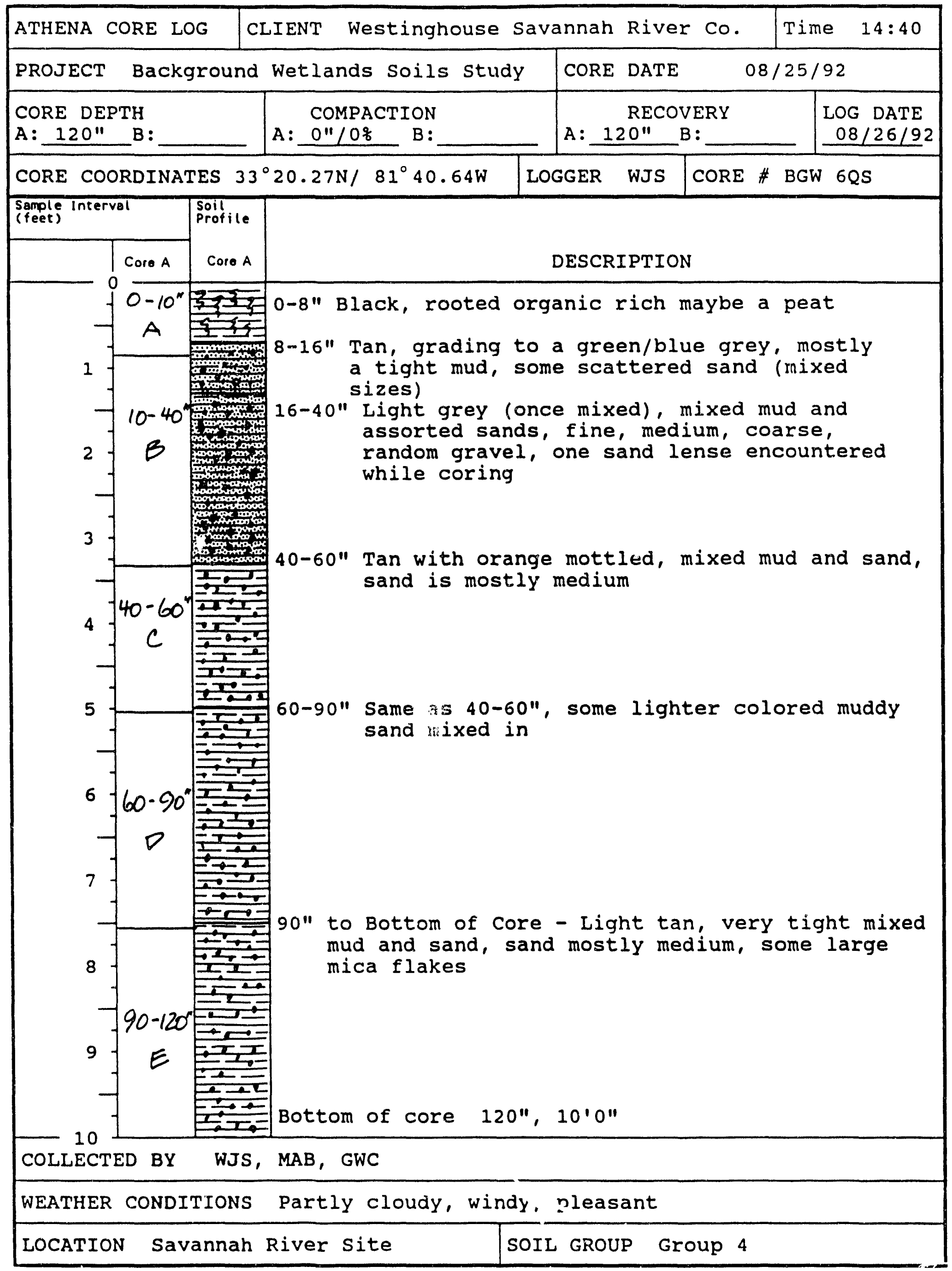




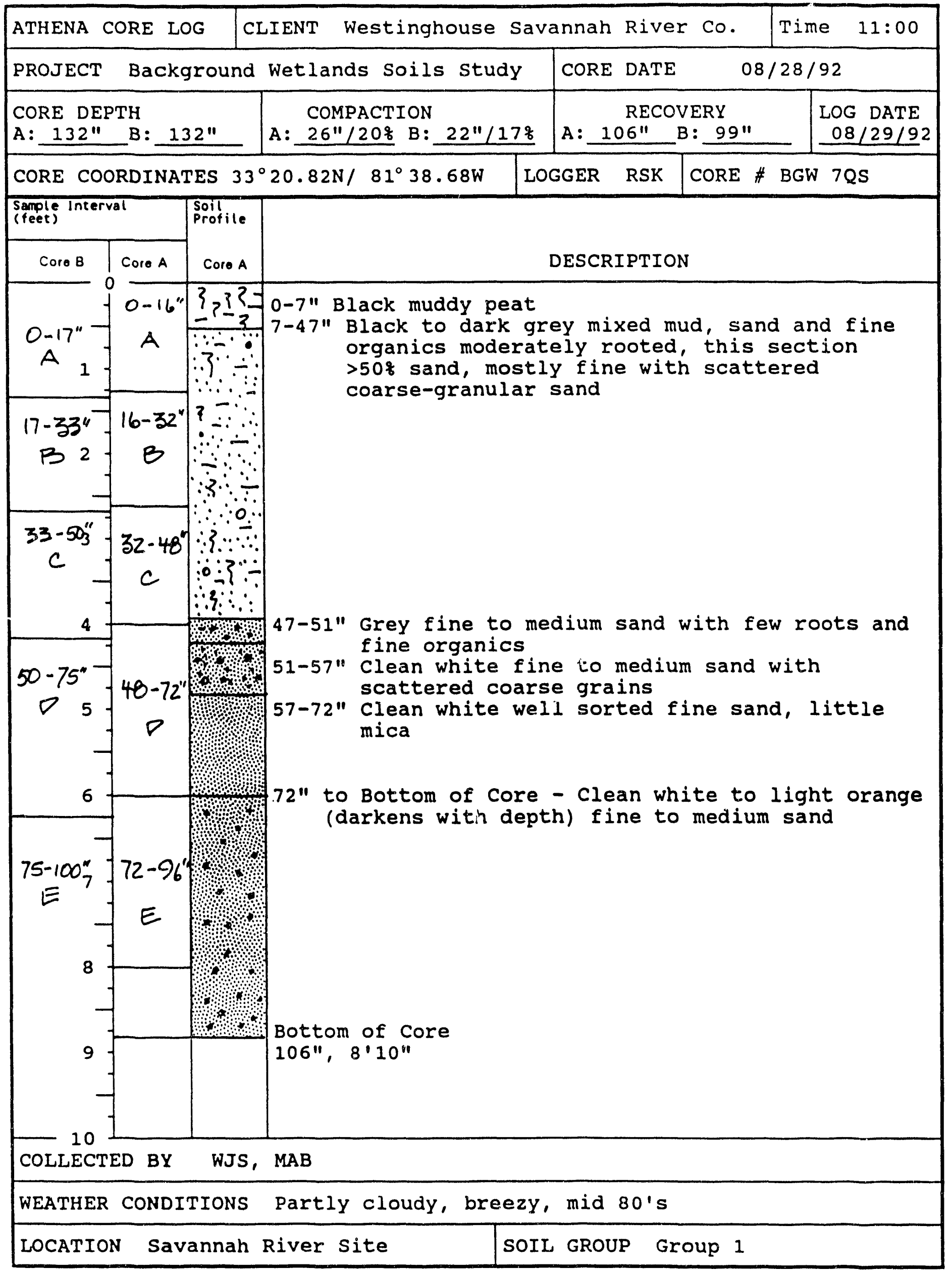




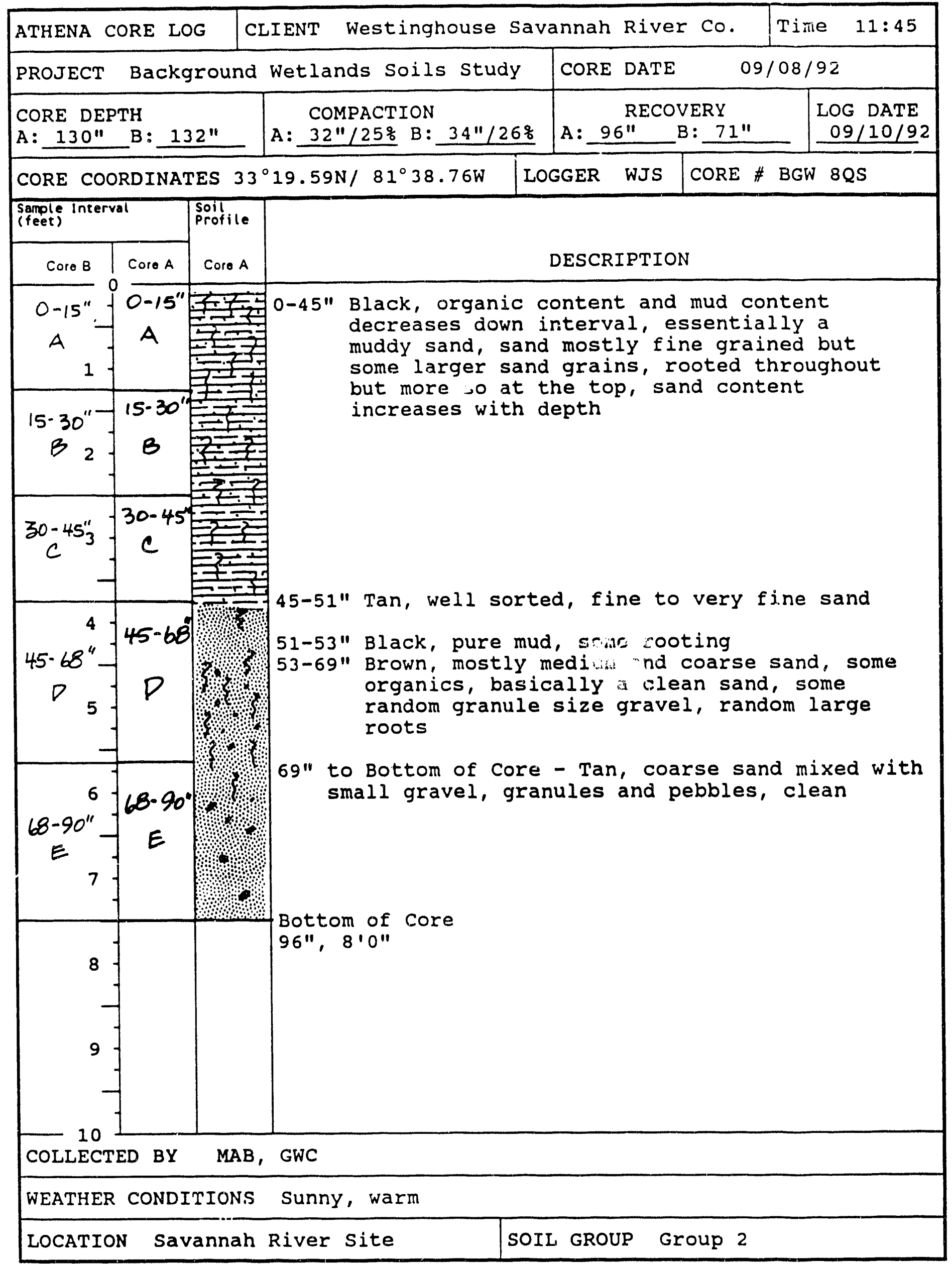


Appendix B

Field Activities Logs 


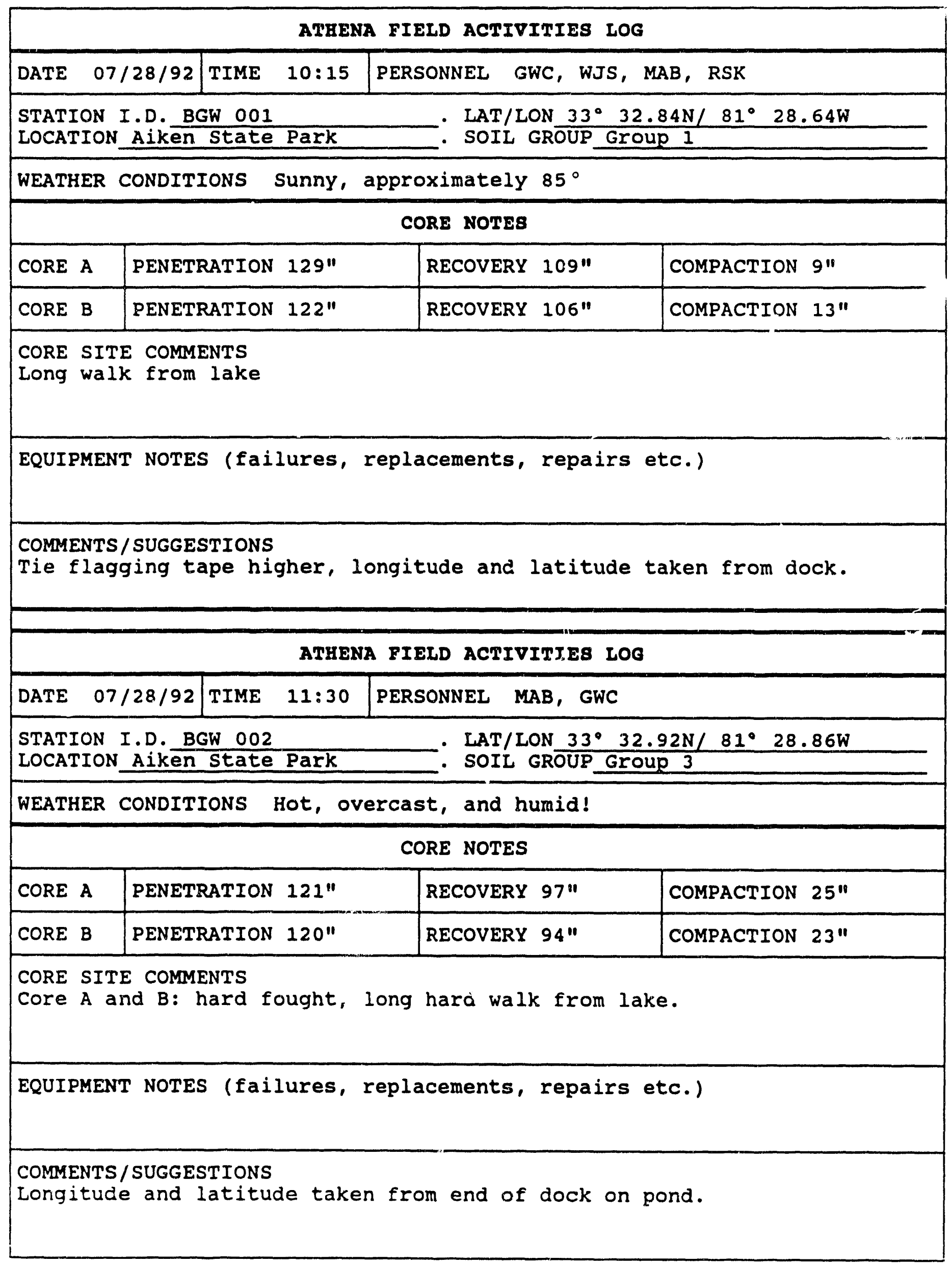




\begin{tabular}{|c|c|c|}
\hline \multicolumn{3}{|c|}{ ATHENA FIELD ACTIVITIES LOG } \\
\hline DATE $07 / 29 / 92$ & \multicolumn{2}{|c|}{ PERSONNEL WJS, MAB, GWC } \\
\hline \multicolumn{3}{|c|}{$\begin{array}{l}\text { STATION I.D. BGW } 003 \\
\text { LOCATION Aiken State Park }\end{array}$} \\
\hline \multicolumn{3}{|c|}{ WEATHER CONDITIONS HOt, clear } \\
\hline \multicolumn{3}{|c|}{ CORE NOTES } \\
\hline PENETRATION 81" & RECOVERY $67 "$ & COMPACTION $12^{\prime \prime}$ \\
\hline PENETRATION $68 "$ & RECOVERY $63 "$ & COMPACTION 9" \\
\hline \multicolumn{3}{|c|}{$\begin{array}{l}\text { CORE SITE COMMENTS } \\
\text { Tight Clay; penetration refused for } A \text { and } B \text {. Hand auger collection of } \\
D \text { and E samples. VOA samples collected on site. }\end{array}$} \\
\hline \multicolumn{3}{|c|}{ EQUIPMENT NOTES (failures, replacements, repairs etc.) } \\
\hline \multicolumn{3}{|l|}{ COMMENTS/SUGGESTIONS } \\
\hline \multicolumn{3}{|c|}{ ATHENA FIELD ACTIVITIEB LOG } \\
\hline DATE $\quad 0 i / 29 / 92$ TIME $\quad 12: 30$ & \multicolumn{2}{|c|}{ PERSONNEL WJS, MAB, GWC } \\
\hline \multicolumn{3}{|c|}{$\begin{array}{ll}\text { STATION I.D. BGW } 004 & \text { LAT/LON } 33^{\circ} 33.28 \mathrm{~N} / 81^{\circ} 29.39 \mathrm{~W} \\
\text { LOCATION Aiken State Park } & \text { SOIL GROUP Group } 4\end{array}$} \\
\hline \multicolumn{3}{|c|}{ WEATHER CONDITIONS Hot, clear } \\
\hline \multicolumn{3}{|c|}{ CORE NOTES } \\
\hline PENETRATION $138 "$ & RECOVERY $127.5^{\prime \prime}$ & COMPACTION $14 "$ \\
\hline PENETRATION 139" & RECOVERY $125^{\prime \prime}$ & COMPACTION 11" \\
\hline \multicolumn{3}{|l|}{ CORE SITE COMMENTS } \\
\hline \multicolumn{3}{|c|}{ EQUIPMENT NOTES (failures, replacements, repairs etc.) } \\
\hline COMMENTS/SUGGESTIONS & & \\
\hline
\end{tabular}




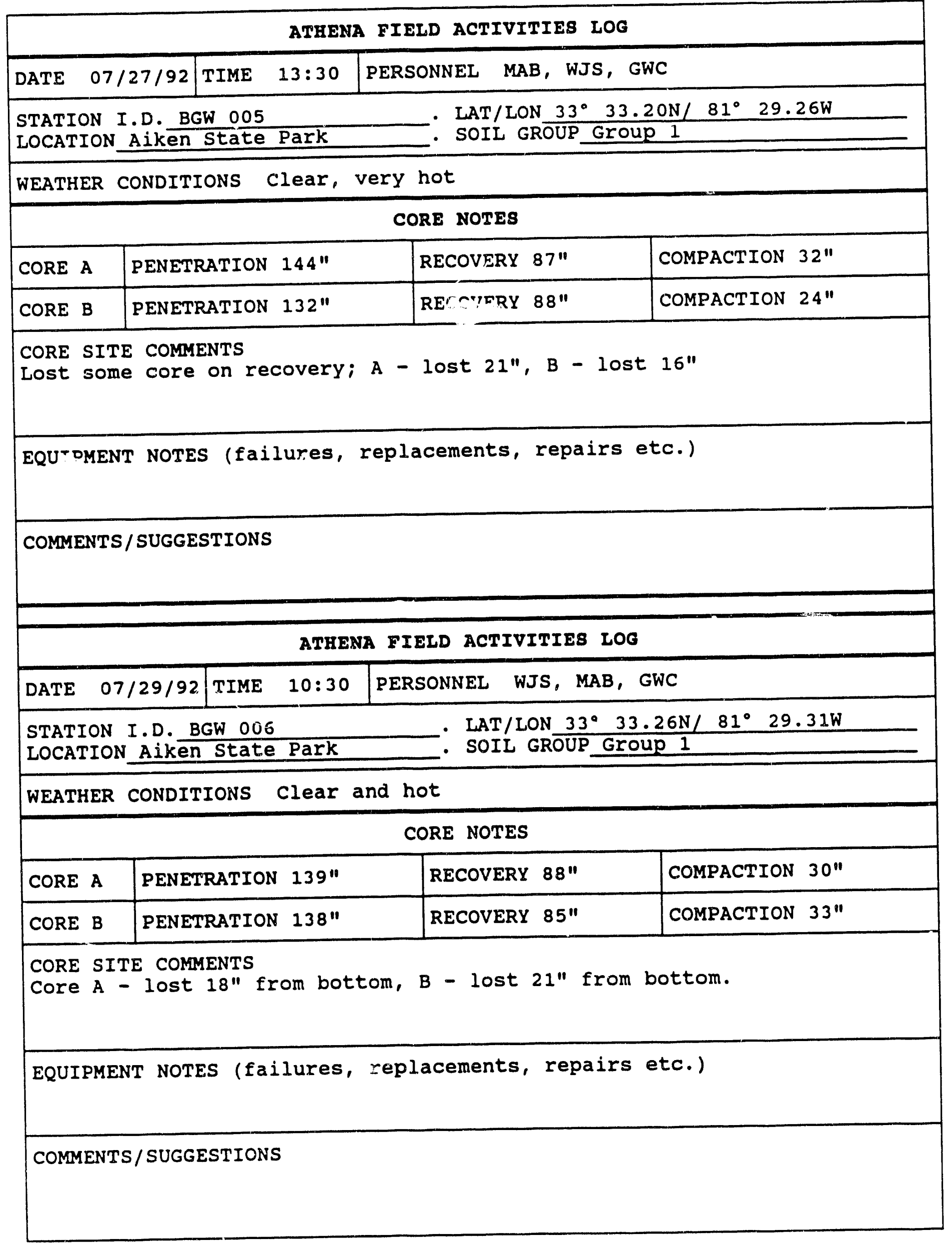




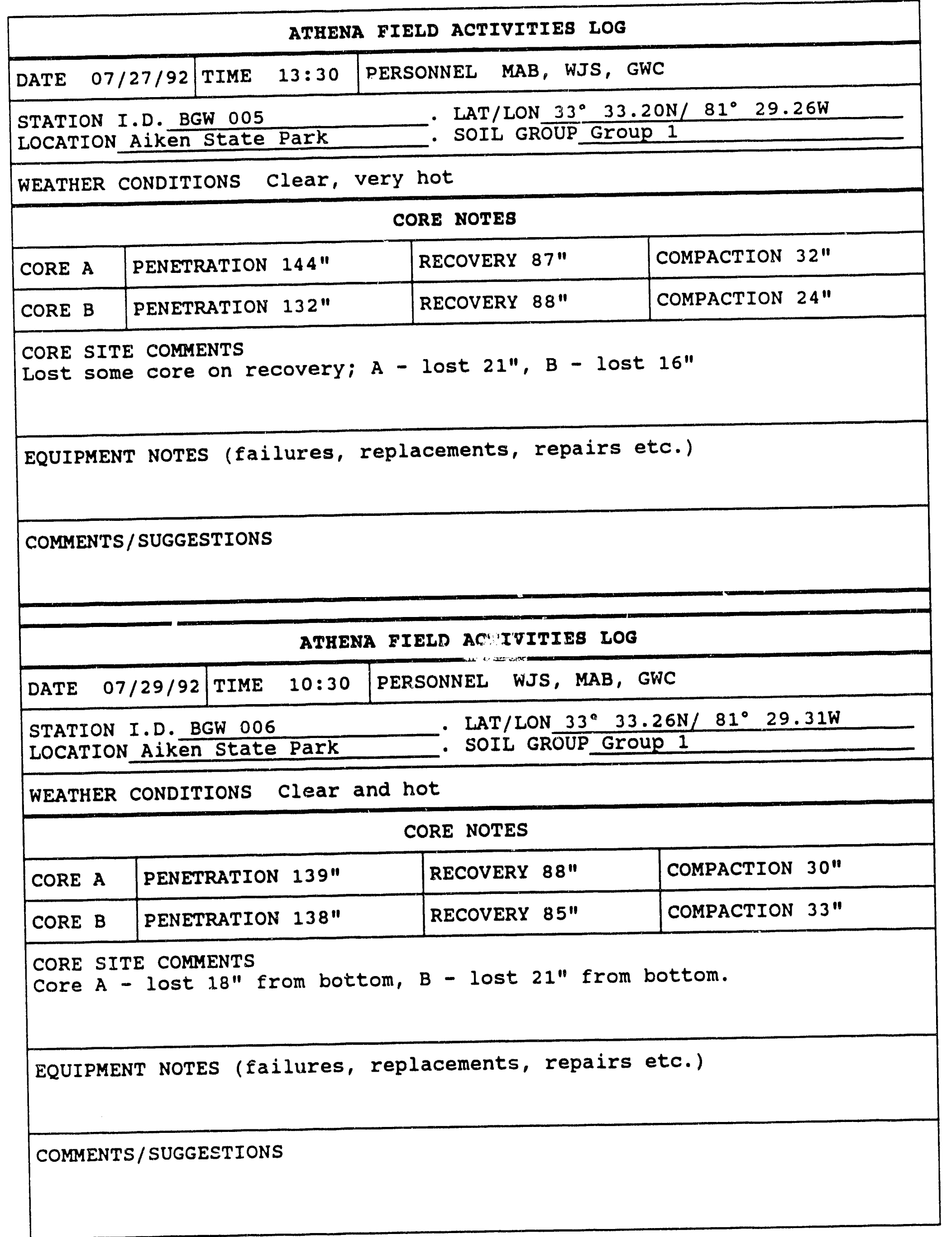




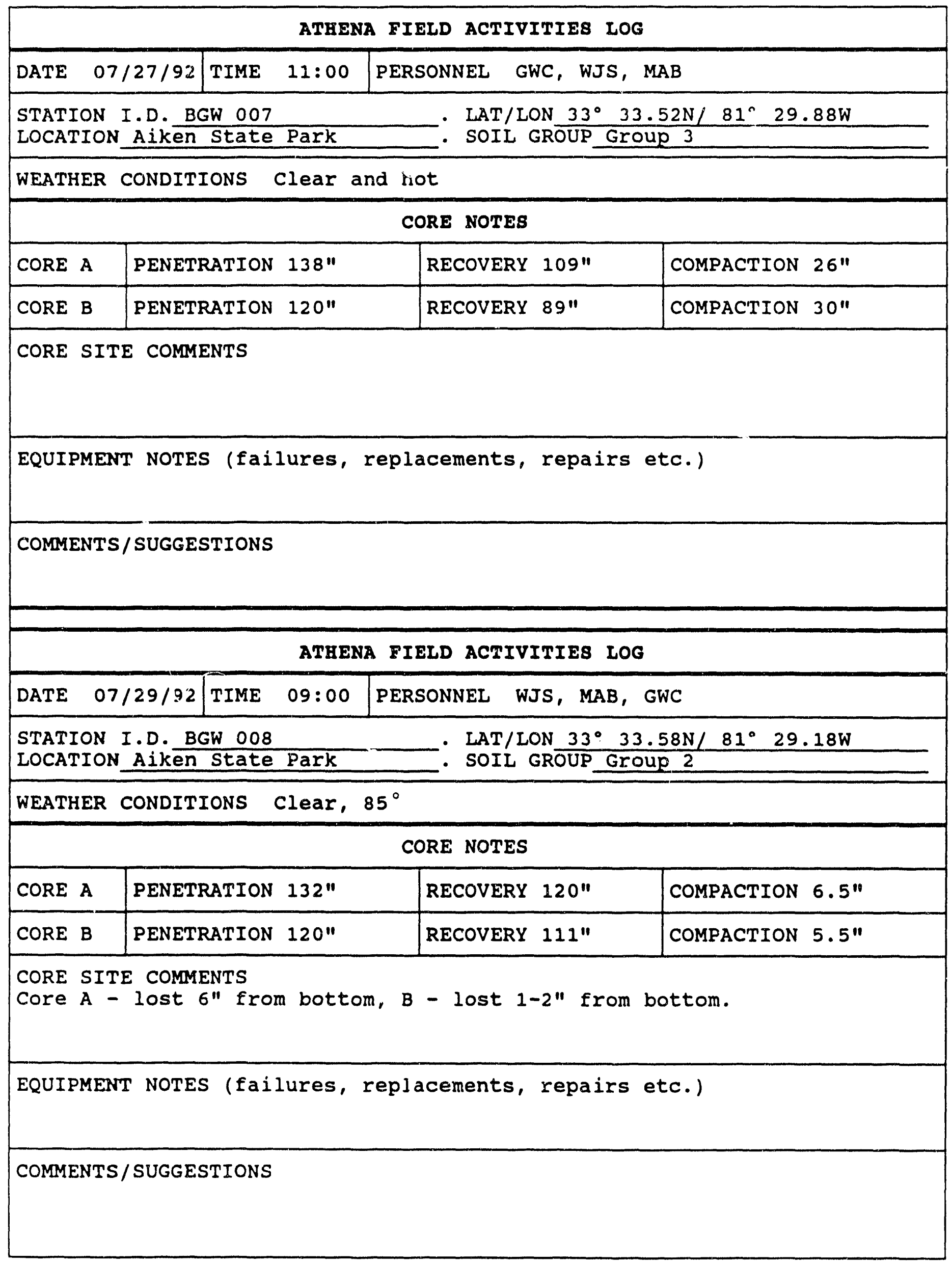




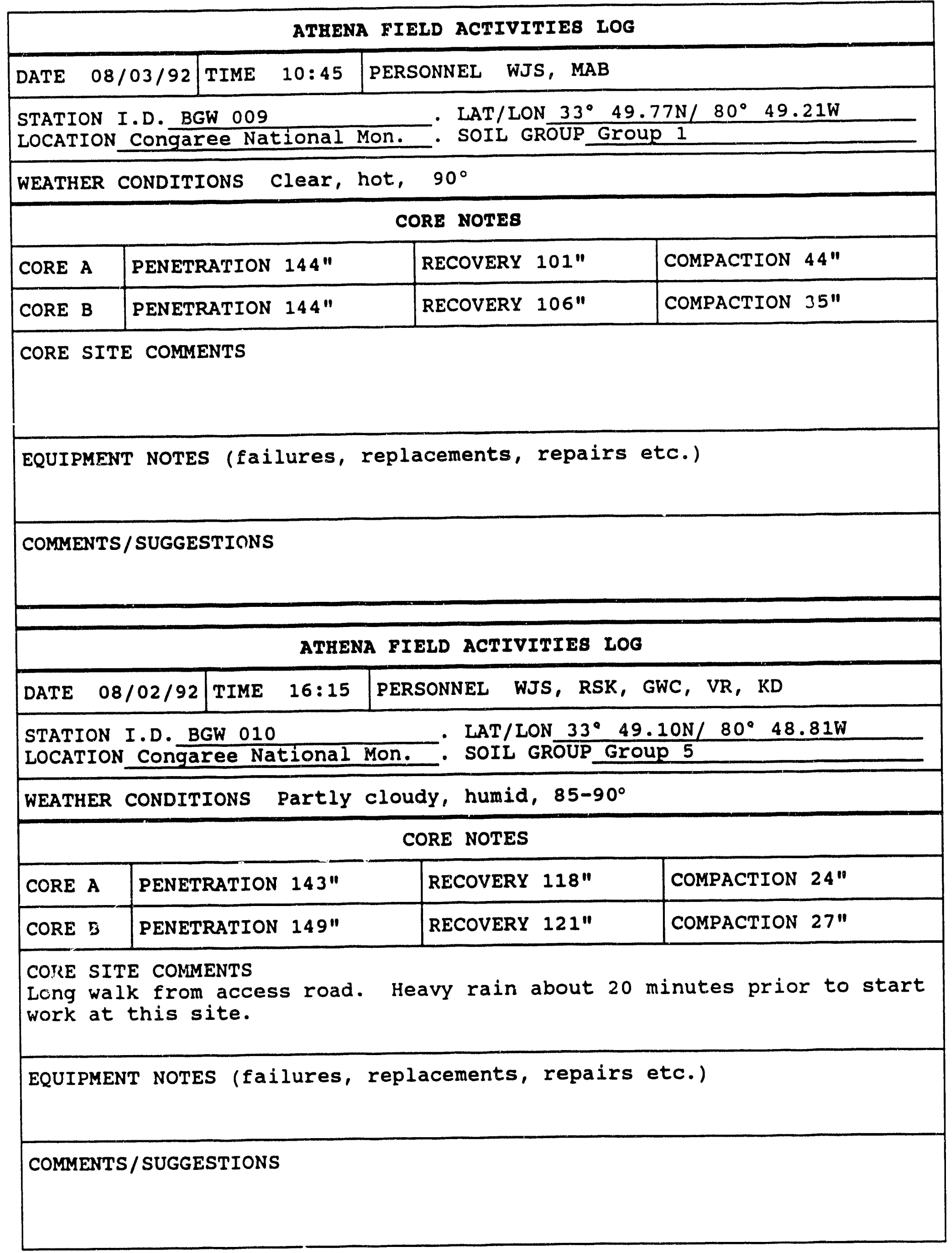




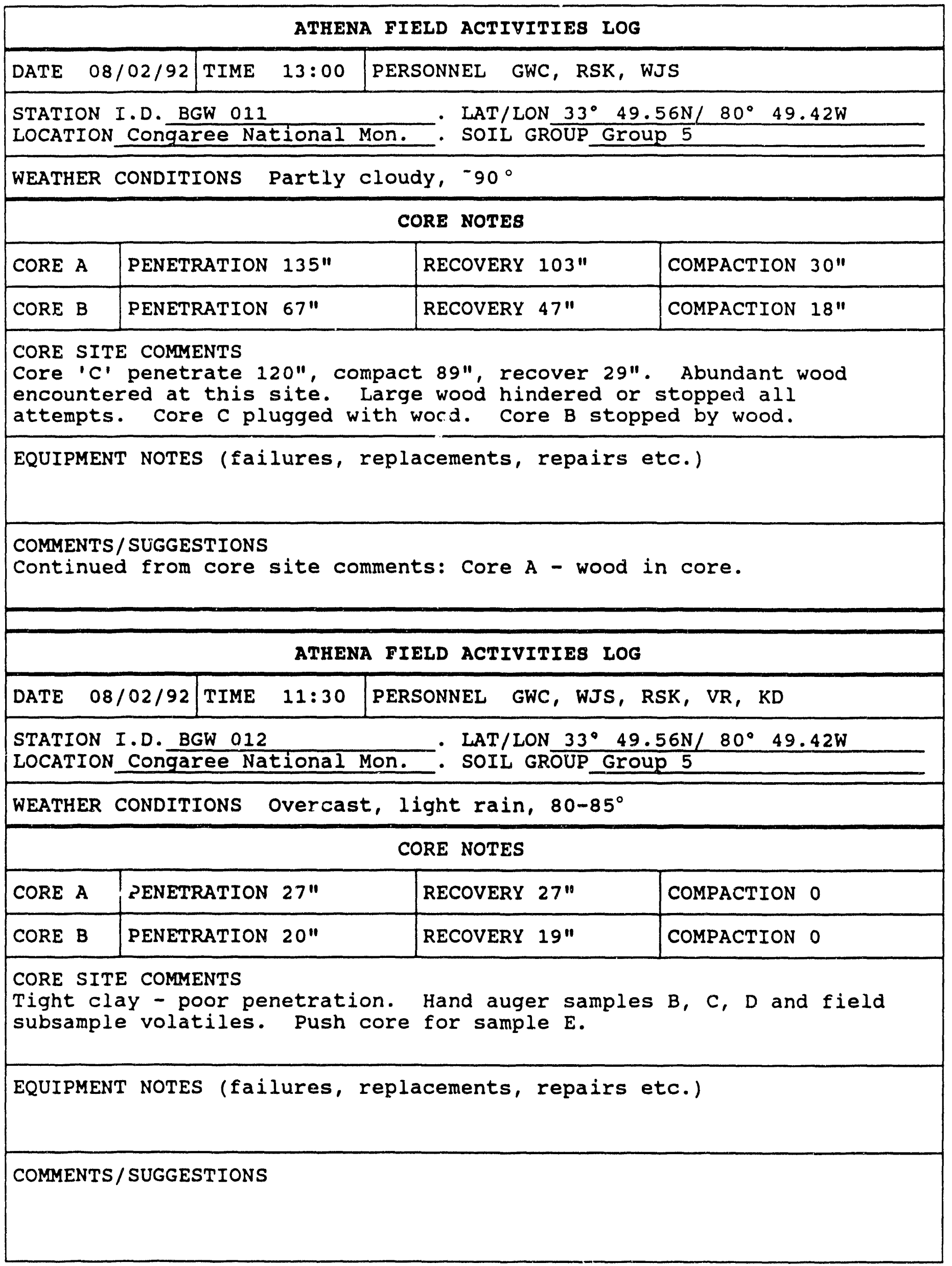




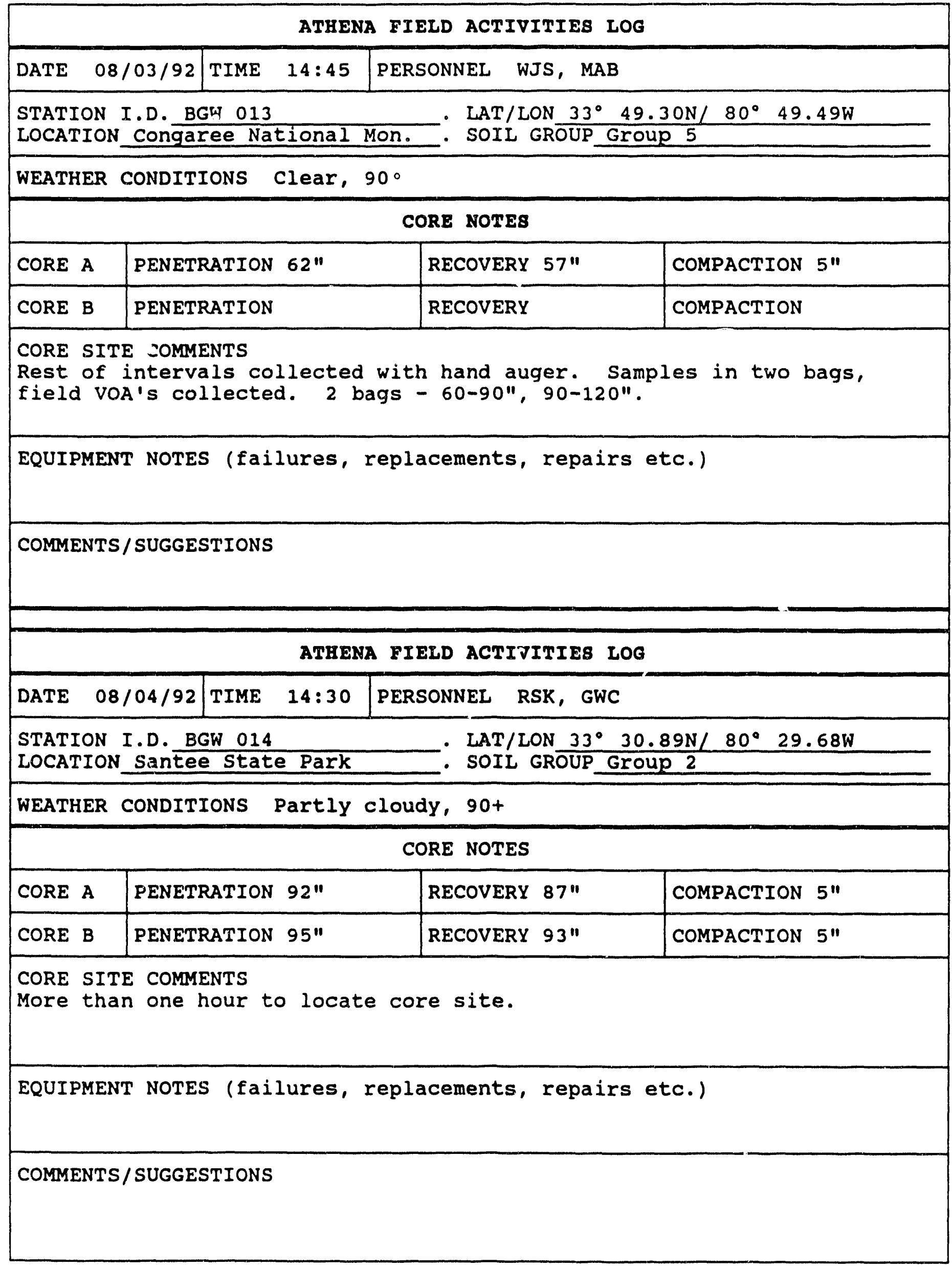




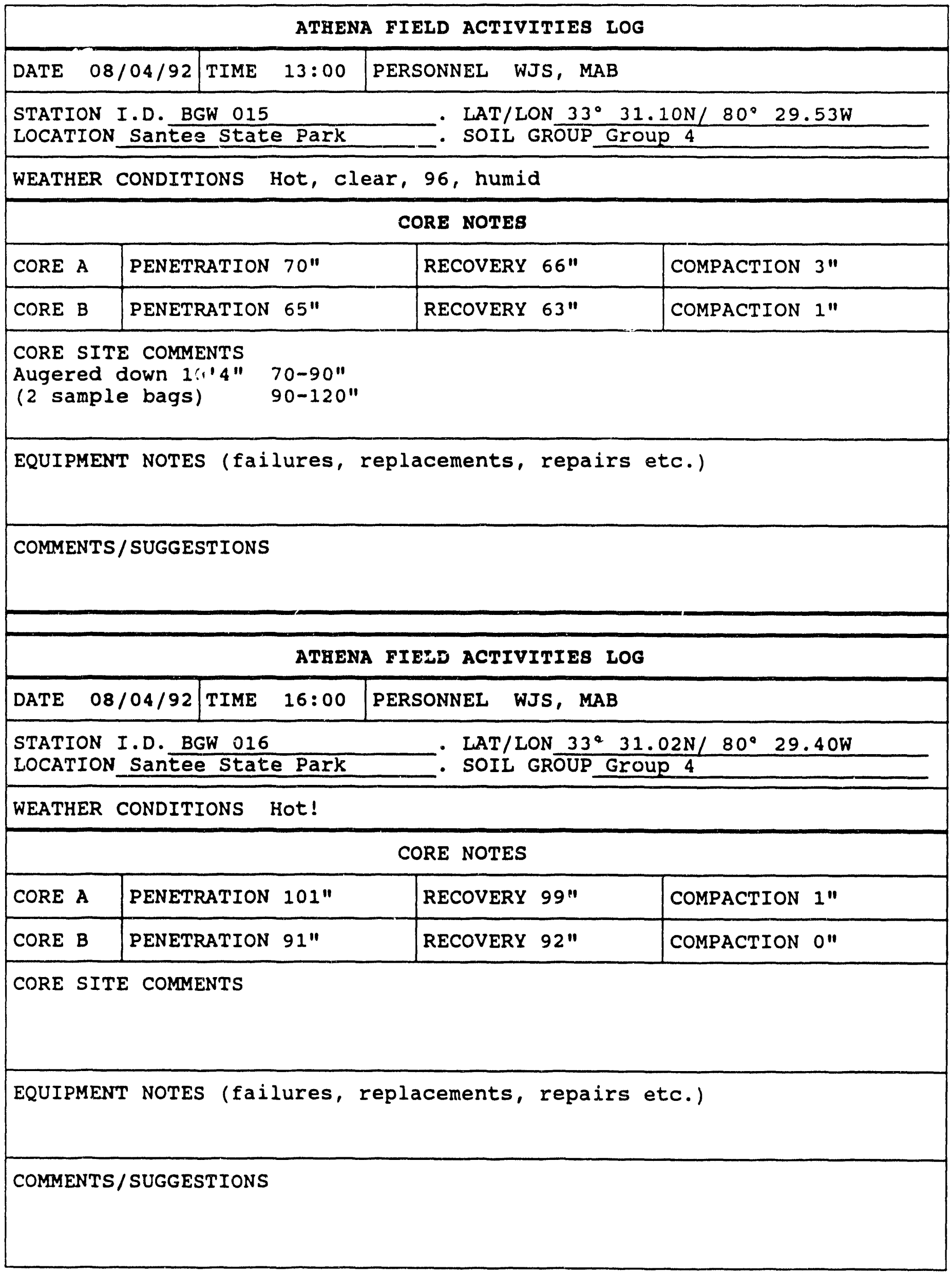




\begin{tabular}{|c|c|c|}
\hline \multicolumn{3}{|c|}{ ATHENA FIELD ACTIVITIES LOG } \\
\hline DATE $08 / 04 / 92$ & PERSONNEL RSK, GWC & \\
\hline \multicolumn{3}{|c|}{$\begin{array}{ll}\text { STATION I.D. BGW } 017 & \text { LAT/LON } 33^{\circ} 31.40 \mathrm{~N} / 80^{\circ} 29.63 \mathrm{~W} \\
\text { LOCATION Santee State Park } & \text {. SOIL GROUP Group } 2\end{array}$} \\
\hline \multicolumn{3}{|l|}{ WEATHER CONDITIONS Clear, $90+$} \\
\hline \multicolumn{3}{|c|}{ CORE NOTES } \\
\hline PENETRATION $58 "$ & RECOVERY $45^{\prime \prime}$ & COMPACTION $14^{\prime \prime}$ \\
\hline PENETRATION $56 "$ & RECOVERY $40 "$ & COMPACTION $14^{\prime \prime}$ \\
\hline \multicolumn{3}{|c|}{$\begin{array}{l}\text { CORE SITE COMMENTS } \\
\text { More than one hour to locate core site. D and E samples collected } \\
\text { using hand auger, field VOA's collected. }\end{array}$} \\
\hline \multicolumn{3}{|c|}{ EQUIPMENT NOTES (failuxes, replacements, repairs etc.) } \\
\hline \multicolumn{3}{|l|}{ COMMENTS/SUGGESTIONS } \\
\hline \multicolumn{3}{|c|}{ ATHENA FIELD ACTIVITIES LOG } \\
\hline \multicolumn{3}{|c|}{\begin{tabular}{|l|l|l|l|l} 
DATE & $08 / 03 / 92$ & TIME $08: 20$ & PERSONNEL WJS, MAB \\
\end{tabular}} \\
\hline \multicolumn{3}{|c|}{ 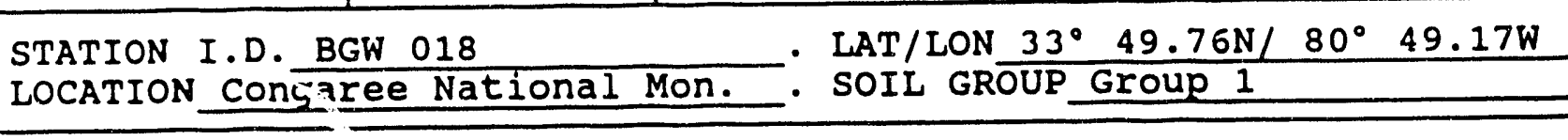 } \\
\hline \multicolumn{3}{|l|}{ WEATHER CONDITIONS Rain } \\
\hline \multicolumn{3}{|c|}{ CORE NOTES } \\
\hline \begin{tabular}{|l|l} 
CORE A & PENETRATION 142" \\
\end{tabular} & RECOVERY 121" & COMPACTION $19 "$ \\
\hline \begin{tabular}{|l|l|} 
CORE B & PENETRATION 142" \\
\end{tabular} & RECOVERY 123" & COMPACTION $19 "$ \\
\hline \multicolumn{3}{|l|}{ CORE SITE COMMENTS } \\
\hline \multicolumn{3}{|c|}{ EQUIPMENT NOTES (failures, replacements, repairs etc.) } \\
\hline COMMENTS/SUGGESTIONS & & \\
\hline
\end{tabular}




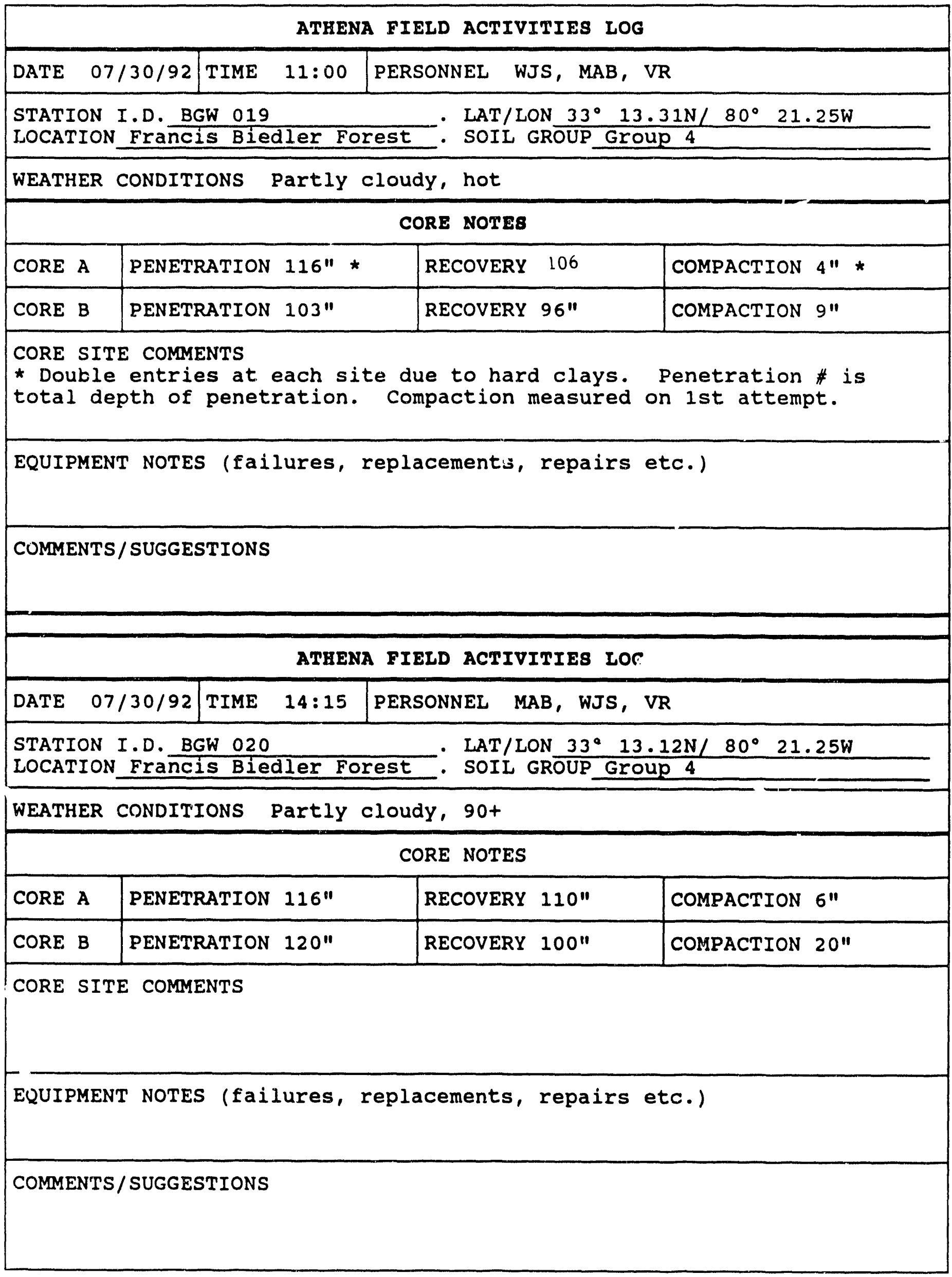




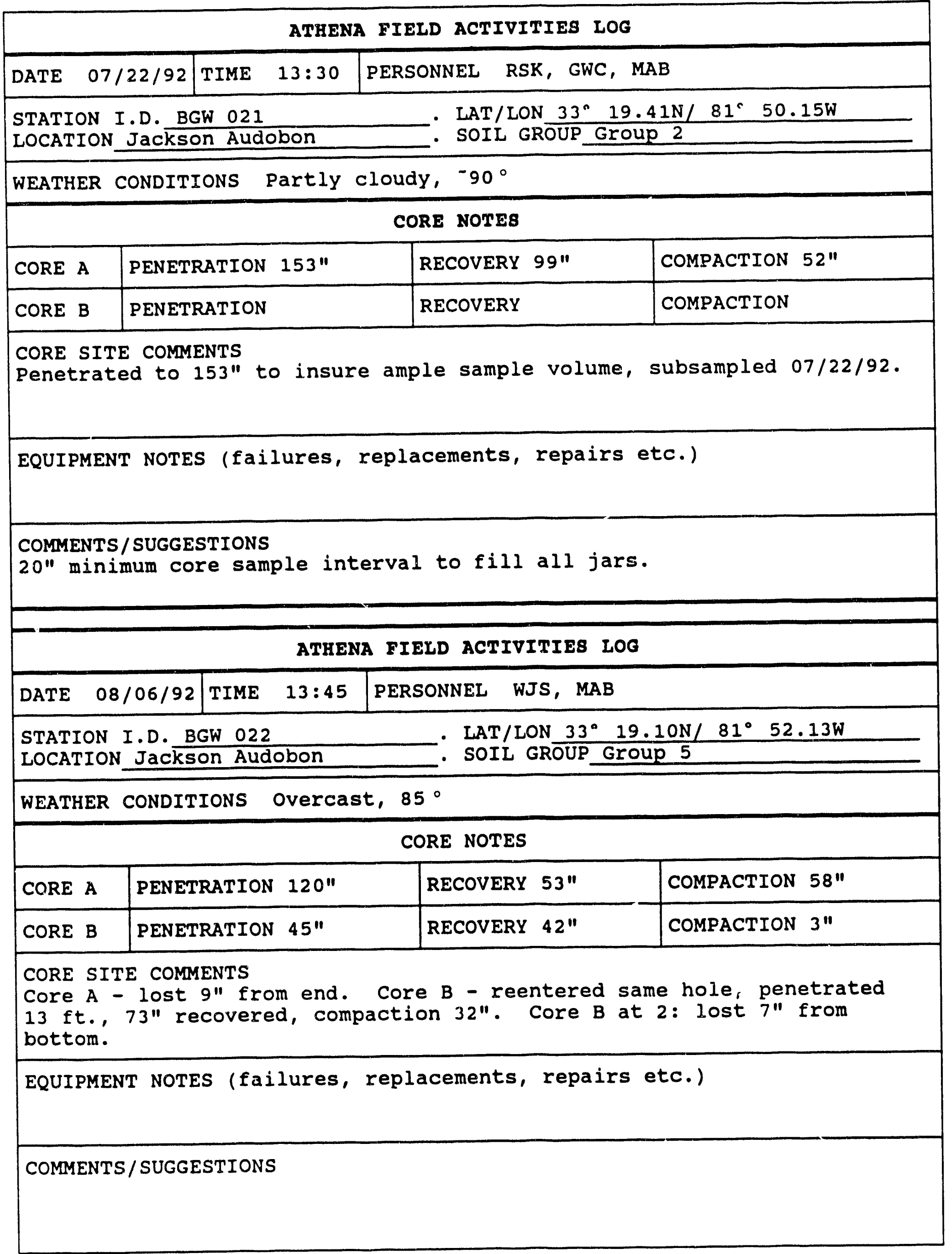




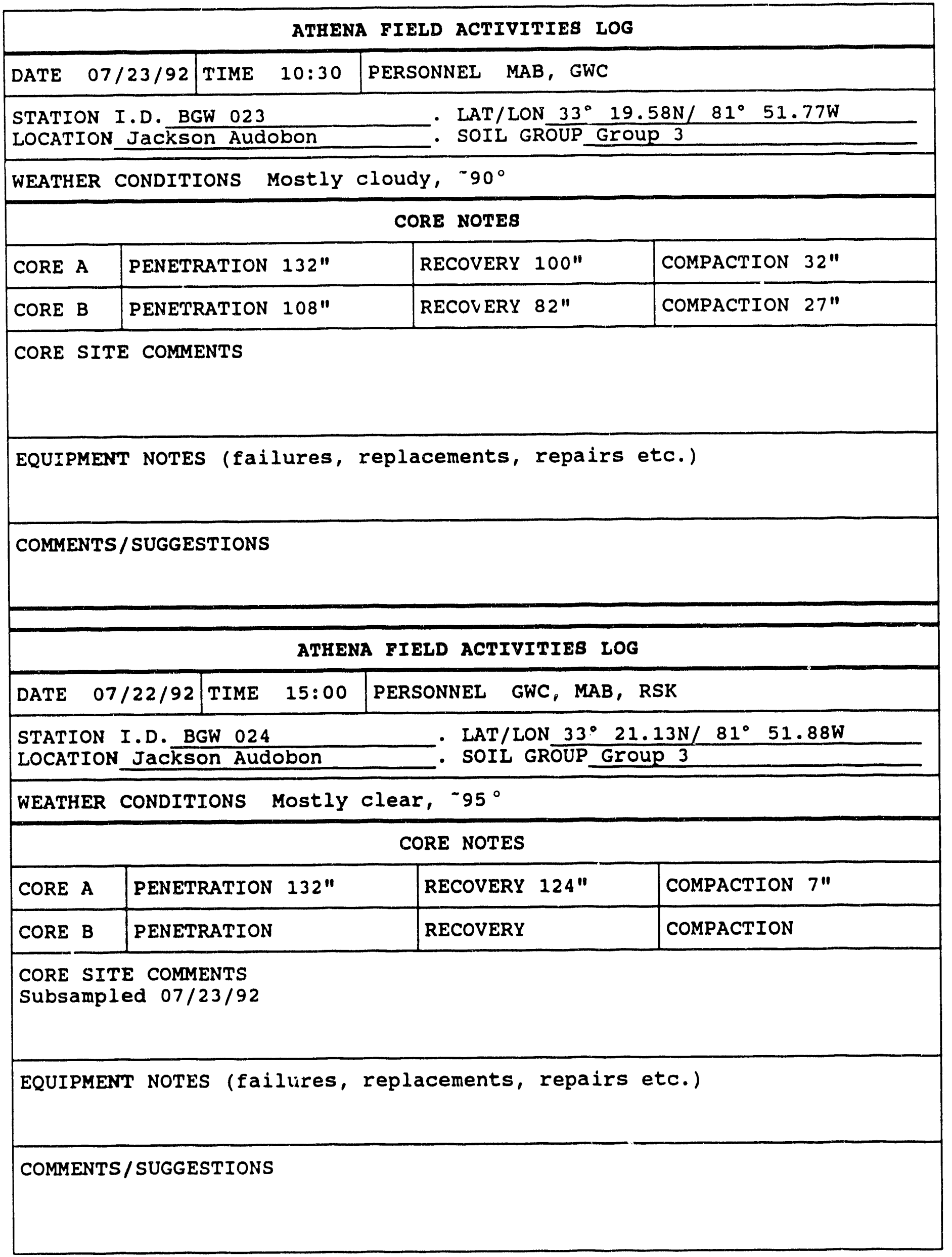









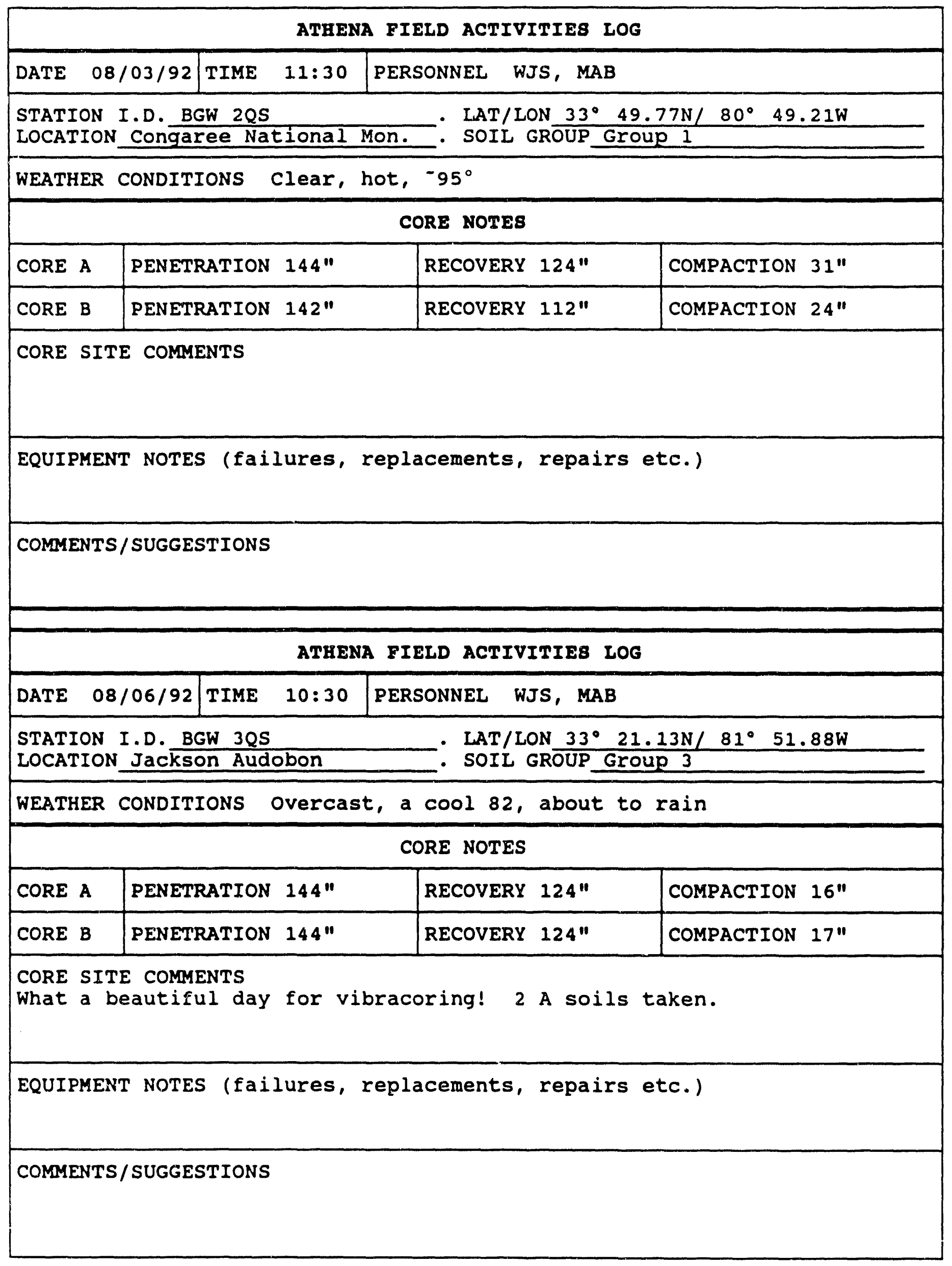




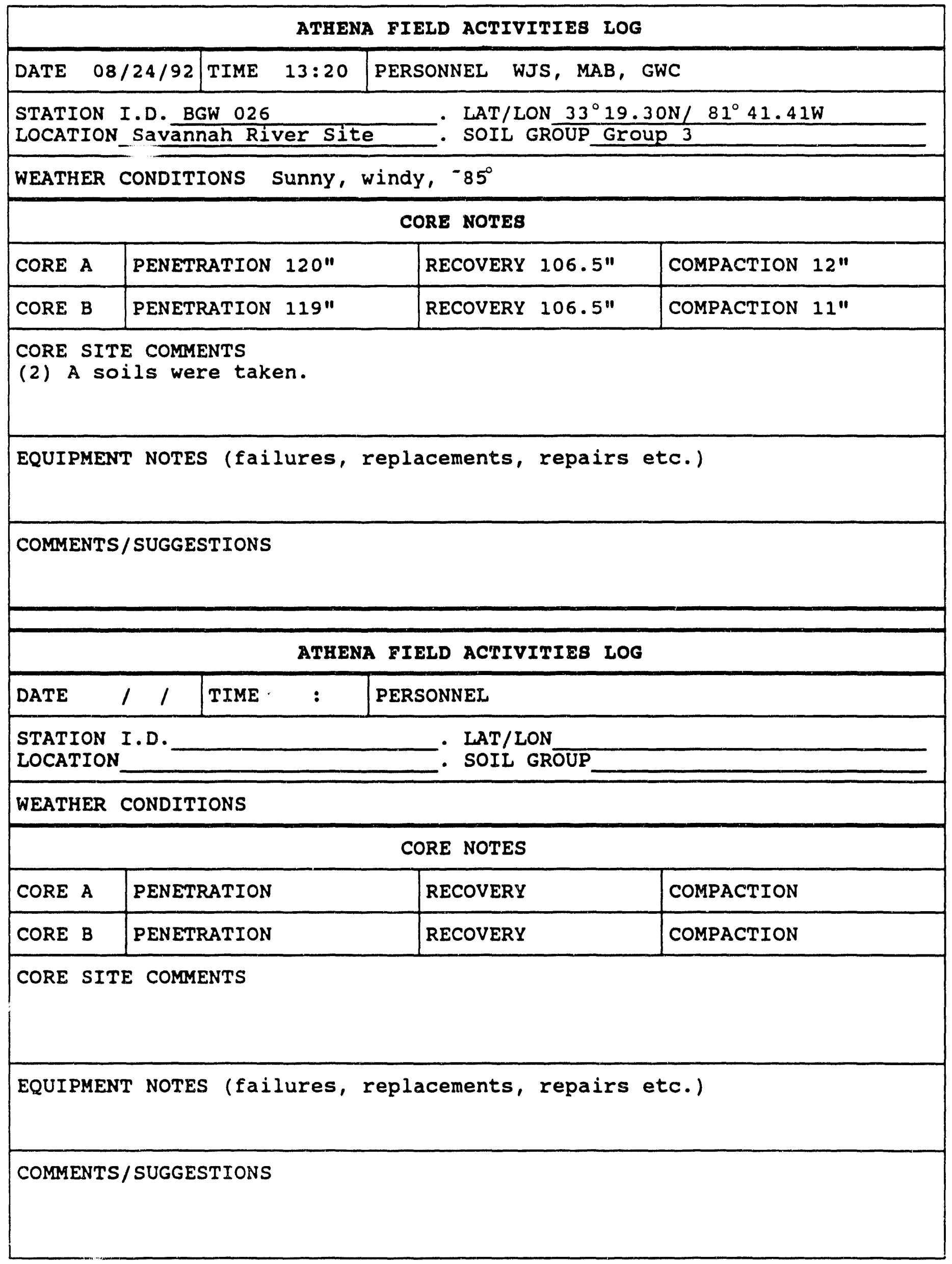




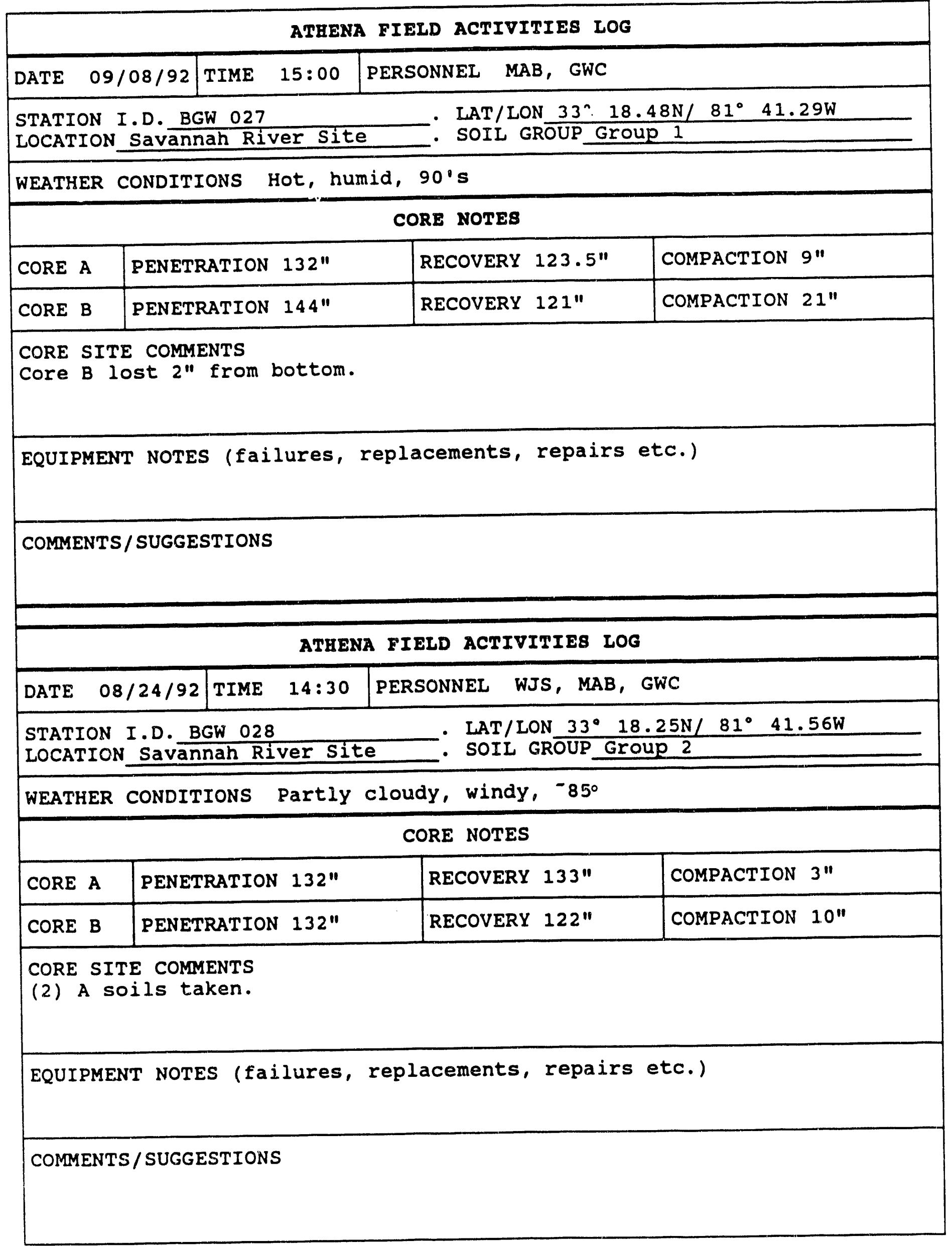




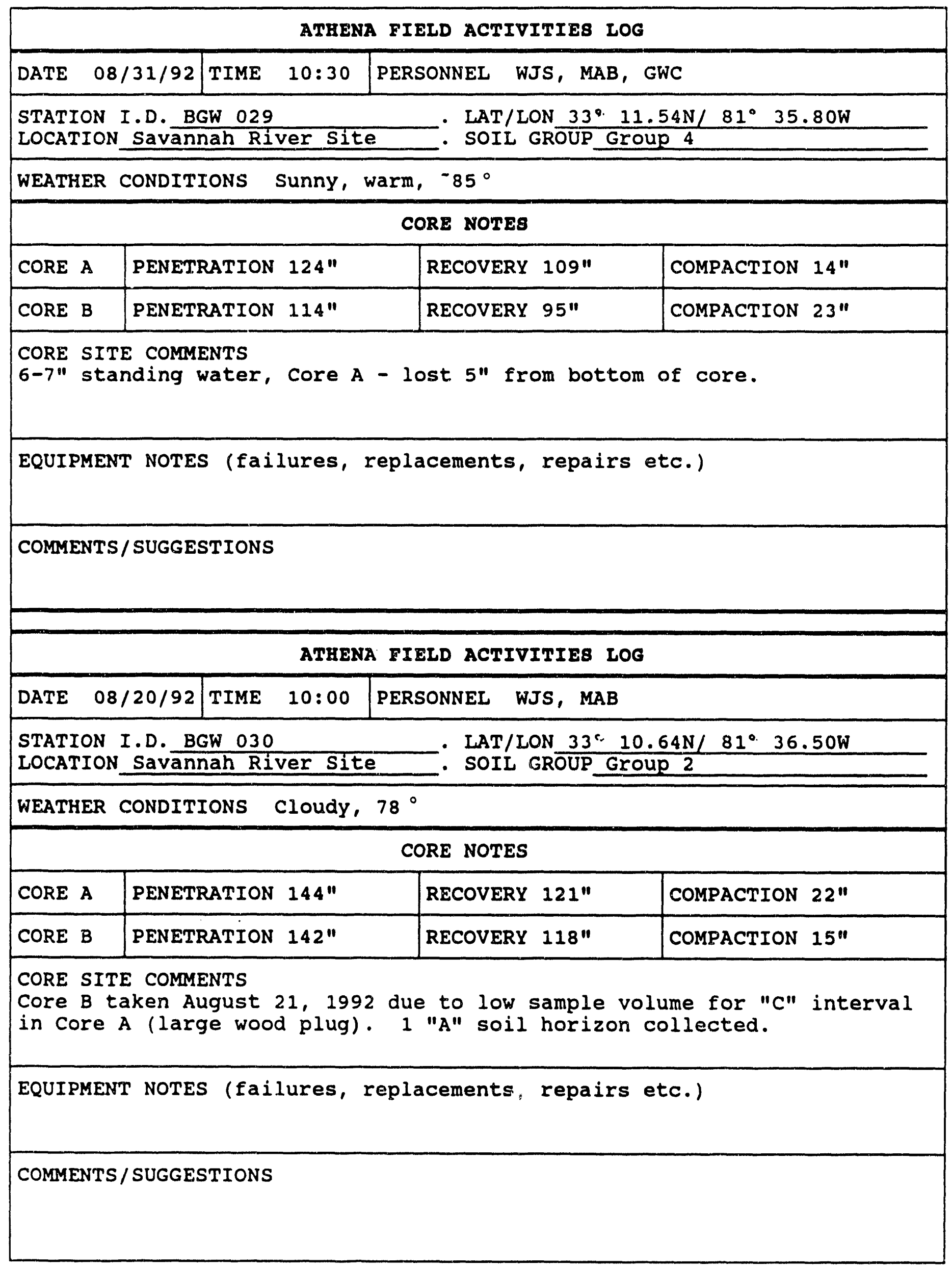




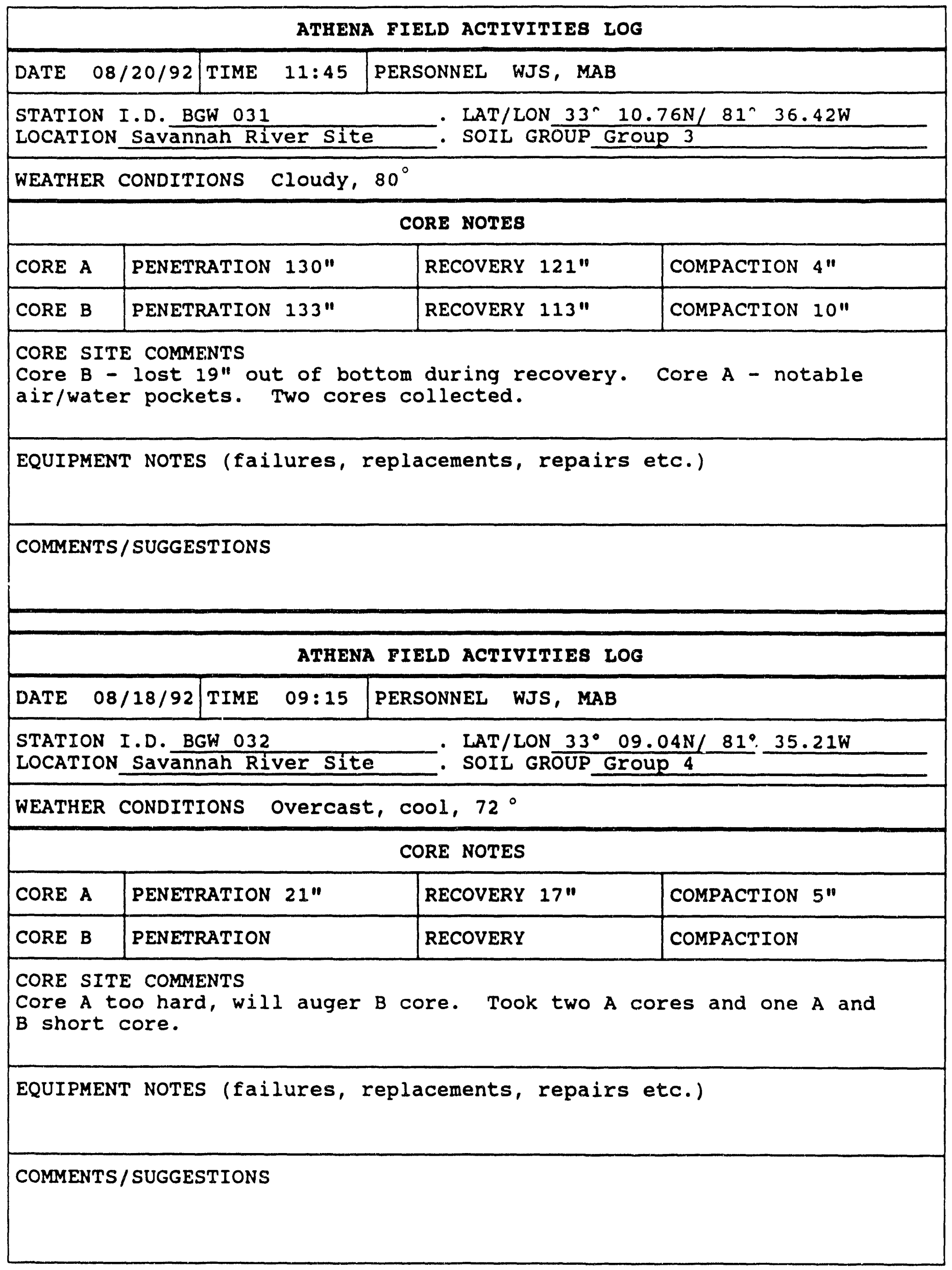




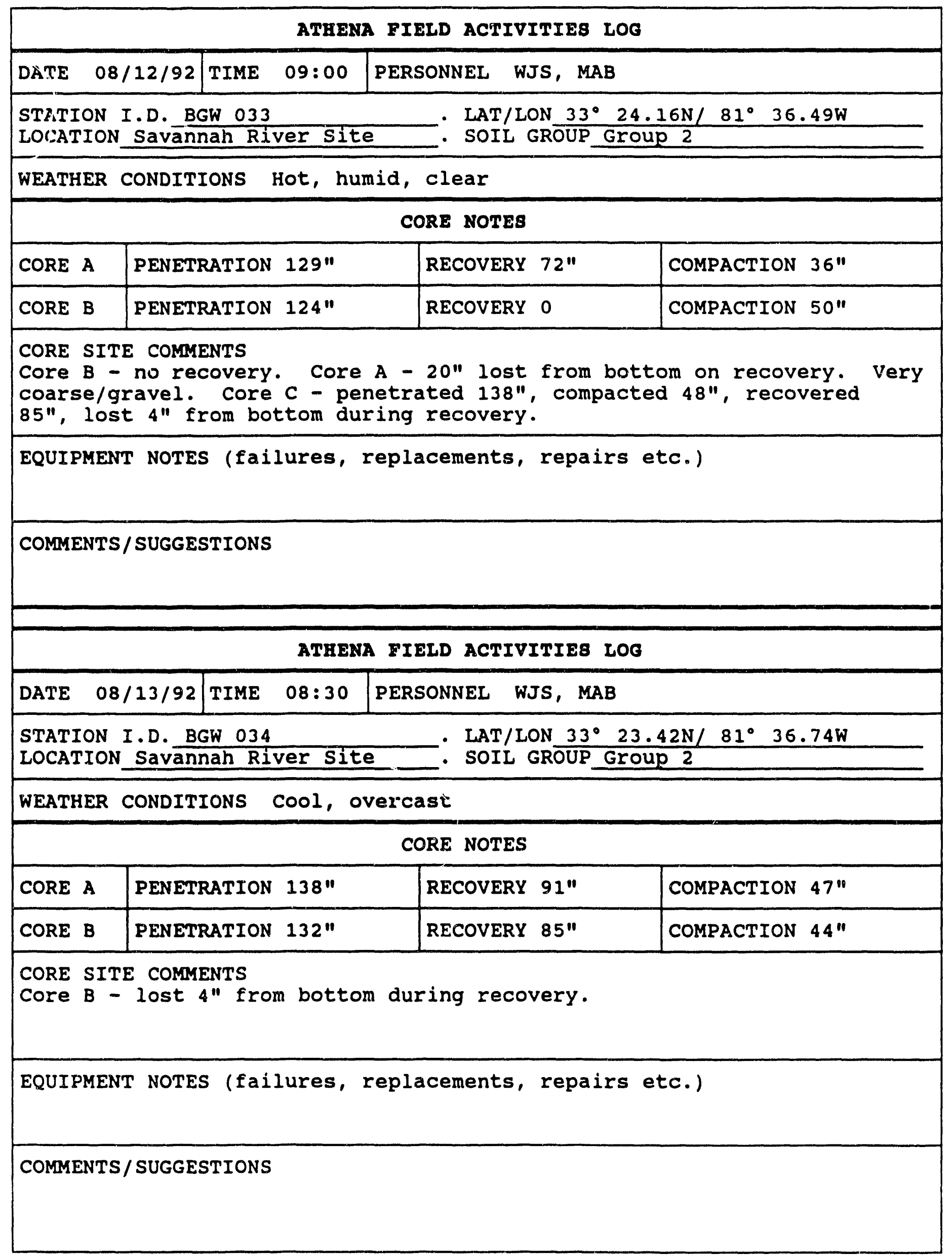









\begin{tabular}{|c|c|c|}
\hline \multicolumn{3}{|c|}{ ATHENA FIELD ACTIVITIES LOG } \\
\hline DATE $08 / 21 / 92$ & \multicolumn{2}{|l|}{ PERSONNEL WJS, MAB } \\
\hline \multicolumn{3}{|c|}{$\begin{array}{l}\text { STATION I.D. BGW } 037 \\
\text { LOCATION Savannah River Site }\end{array}$} \\
\hline \multicolumn{3}{|c|}{ WEATHER CONDITIONS ClOUdY, $-70^{\circ}$} \\
\hline \multicolumn{3}{|c|}{ CORE NOTES } \\
\hline PENETRATION $139 "$ & RECOVERY 61" & COMPACTION $45^{\prime \prime}$ \\
\hline PENETRATION $140 "$ & RECOVERY $90^{\prime \prime}$ & COMPACTION $48^{\prime \prime}$ \\
\hline \multicolumn{3}{|c|}{$\begin{array}{l}\text { CORE SITE COMMENTS } \\
2 \text { cores and } 2 \text { "A" soil horizons collected. Core A - lost } 32 \text { " from } \\
\text { bottom. Core B - lost 1" from bottom. }\end{array}$} \\
\hline \multicolumn{3}{|c|}{ EQUIPMENT NOTES (failures, replacements, repairs etc.) } \\
\hline \multicolumn{3}{|l|}{ COMMENTS/SUGGESTIONS } \\
\hline \multicolumn{3}{|c|}{ ATHENA FIELD ACTIVITIES LOG } \\
\hline \multicolumn{3}{|c|}{\begin{tabular}{|l|l|l|ll} 
DATE & $08 / 24 / 92$ & TIME $10: 15$ & PERSONNEL WJS, MAB \\
\end{tabular}} \\
\hline \multicolumn{3}{|c|}{ 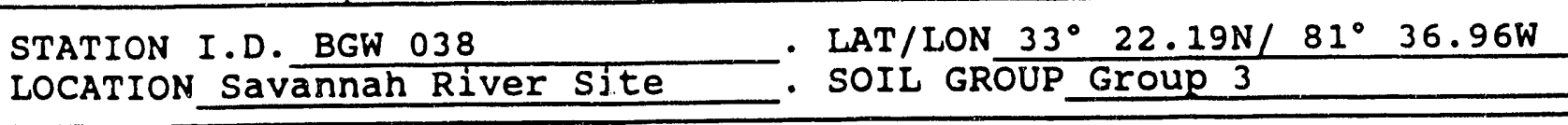 } \\
\hline \multicolumn{3}{|c|}{ WEATHER CONDITIONS Cloudy, cool } \\
\hline \multicolumn{3}{|c|}{ CORE NOTES } \\
\hline PENETRATION $115^{\prime \prime}$ & RECOVERY $102 "$ & COMPACTION $15^{\prime \prime}$ \\
\hline CORE B PENETRATION $120 "$ & RECOVERY 107" & COMPACTION $12 "$ \\
\hline \multicolumn{3}{|l|}{$\begin{array}{l}\text { CORE SITE COMMENTS } \\
\text { (2) A soils were taken. }\end{array}$} \\
\hline \multicolumn{3}{|c|}{ EQUIPMENT NOTES (failures, replacements, repairs etc.) } \\
\hline COMMENTS/SUGGESTIONS & & \\
\hline
\end{tabular}




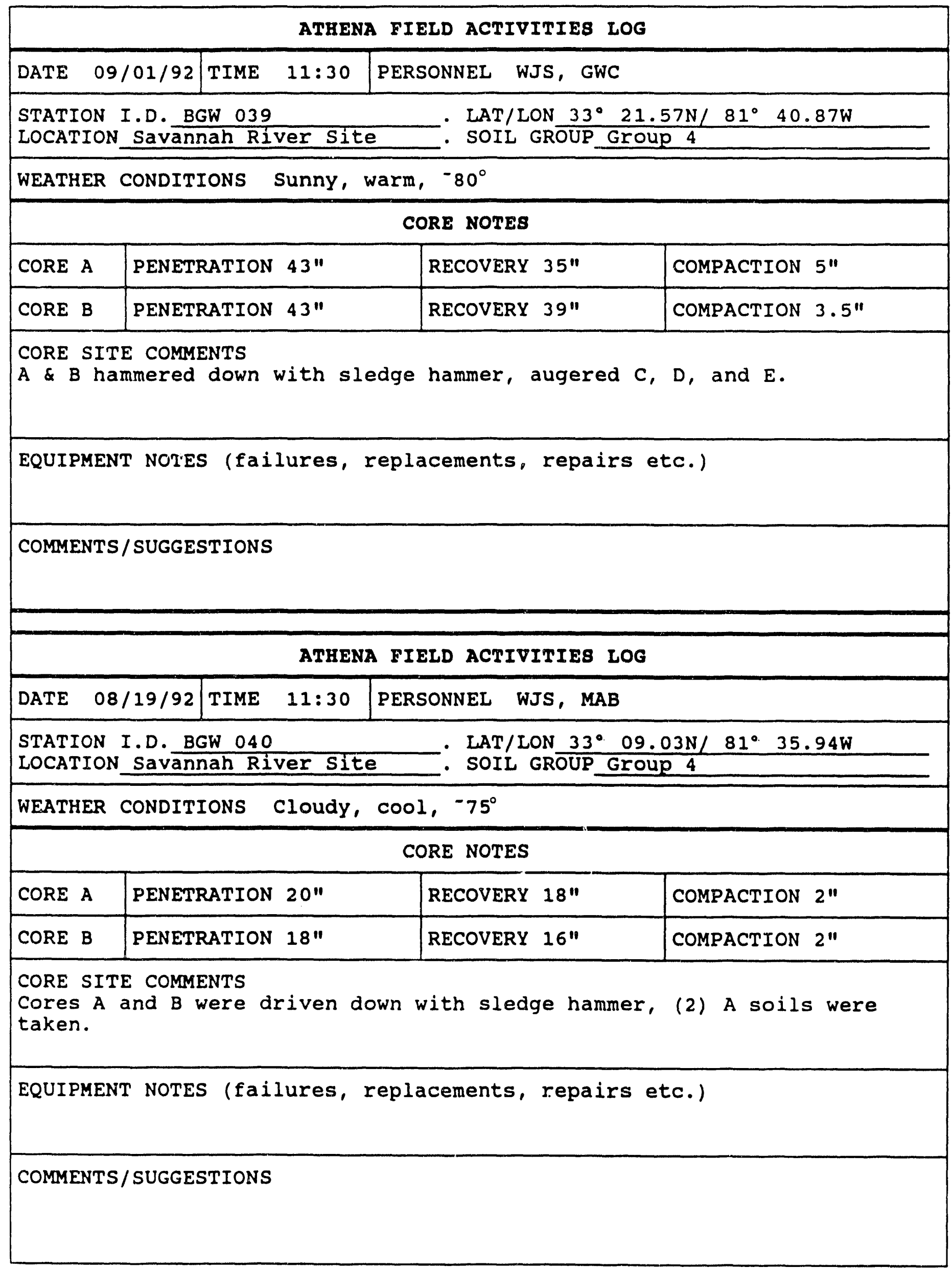




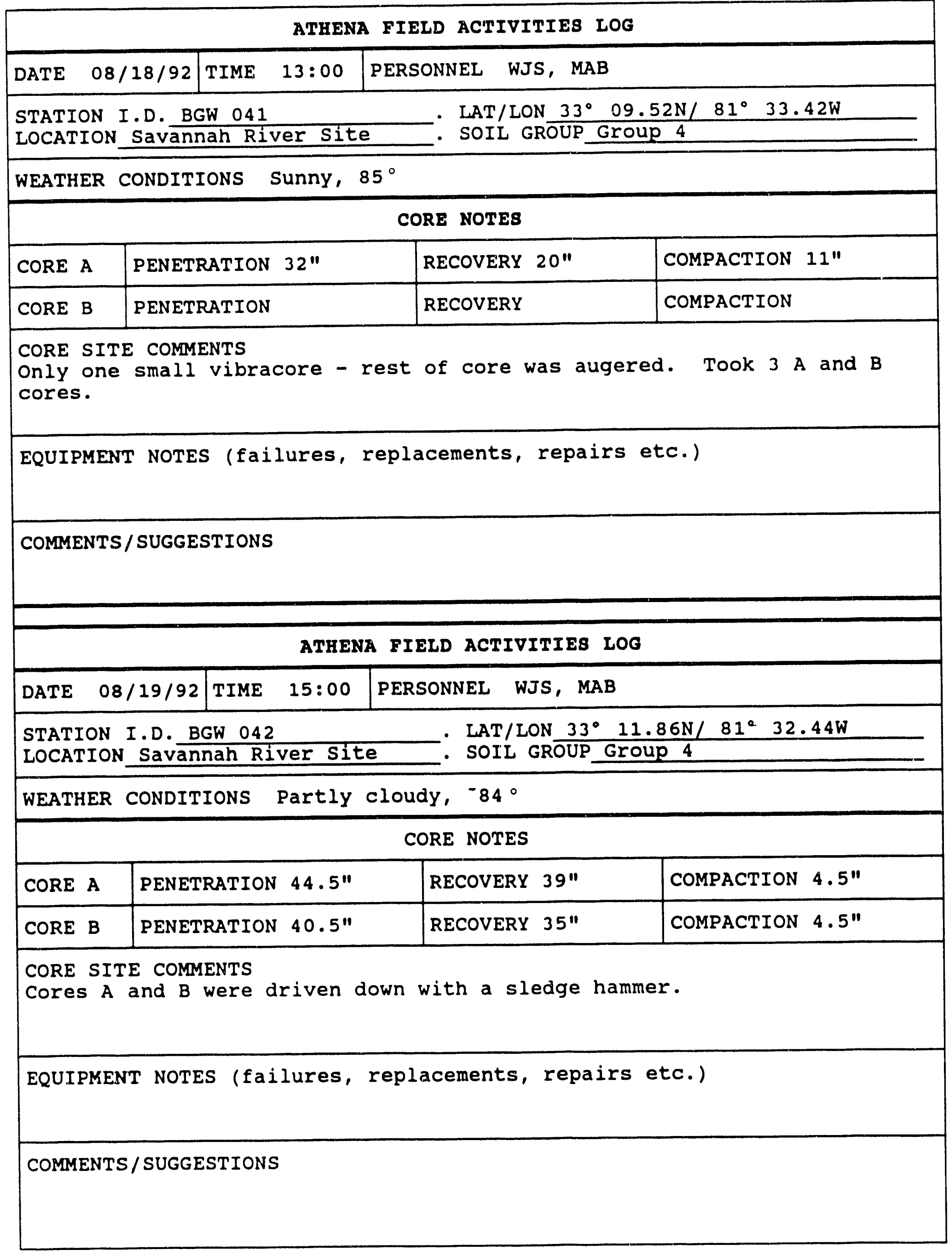




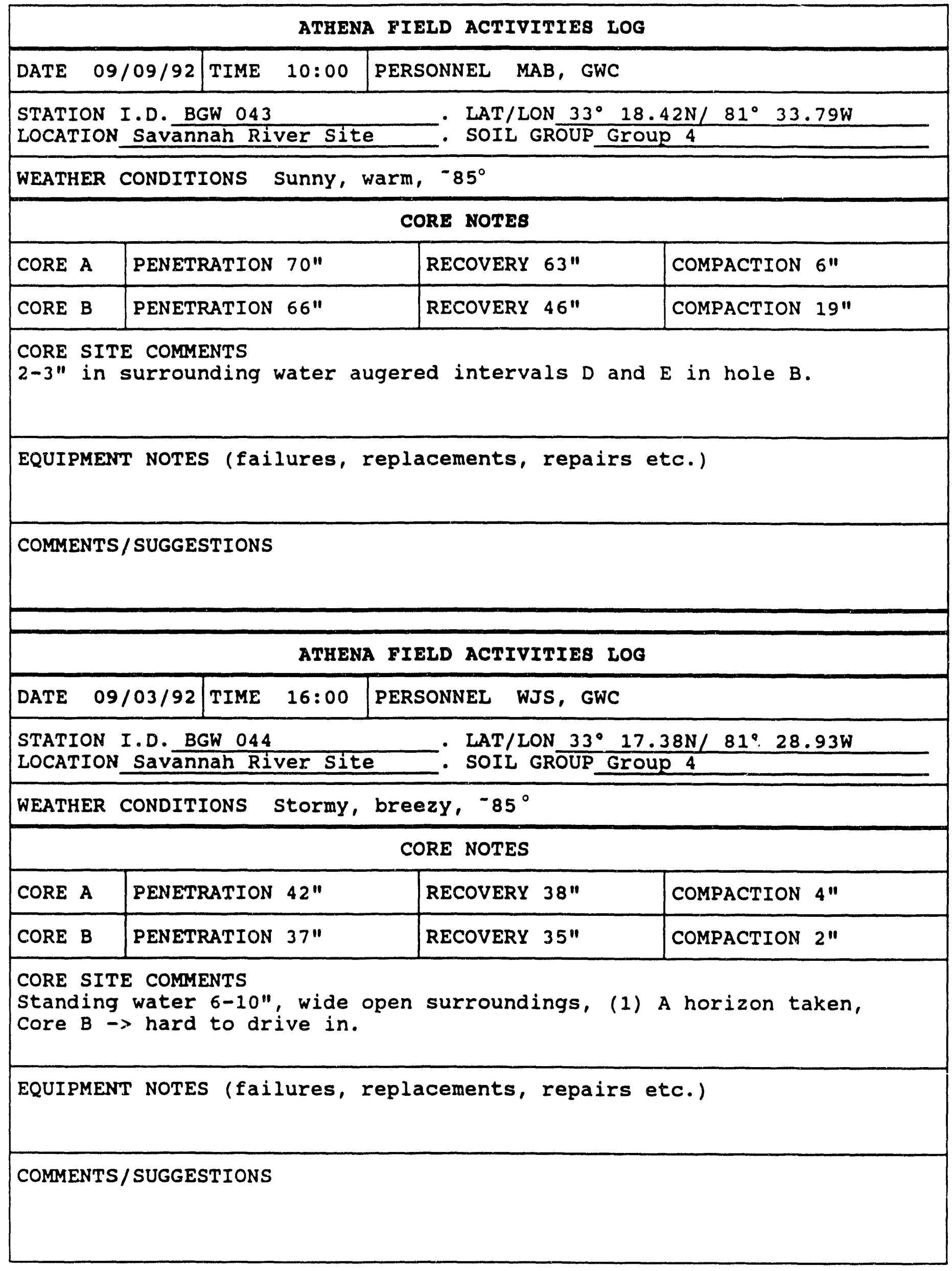




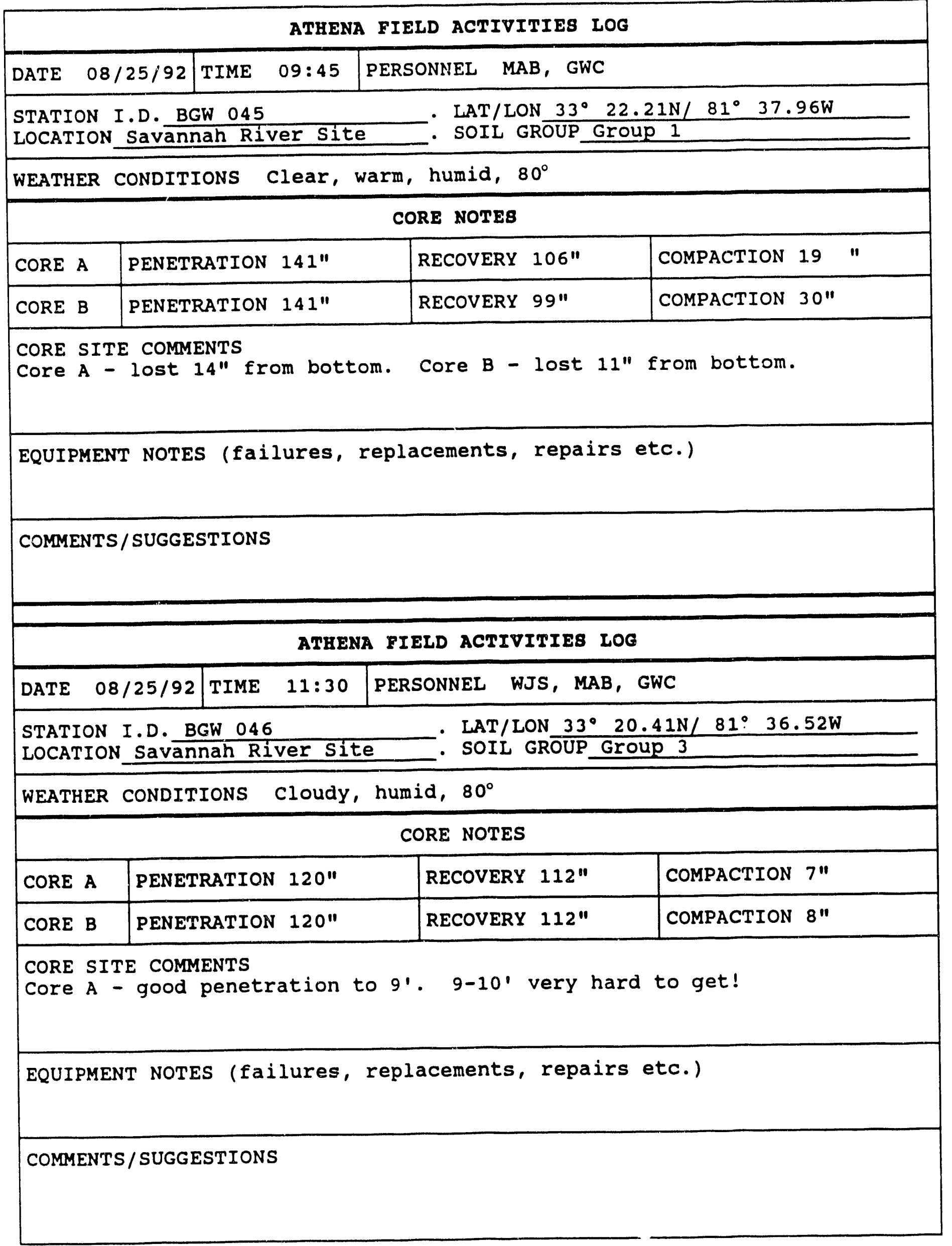




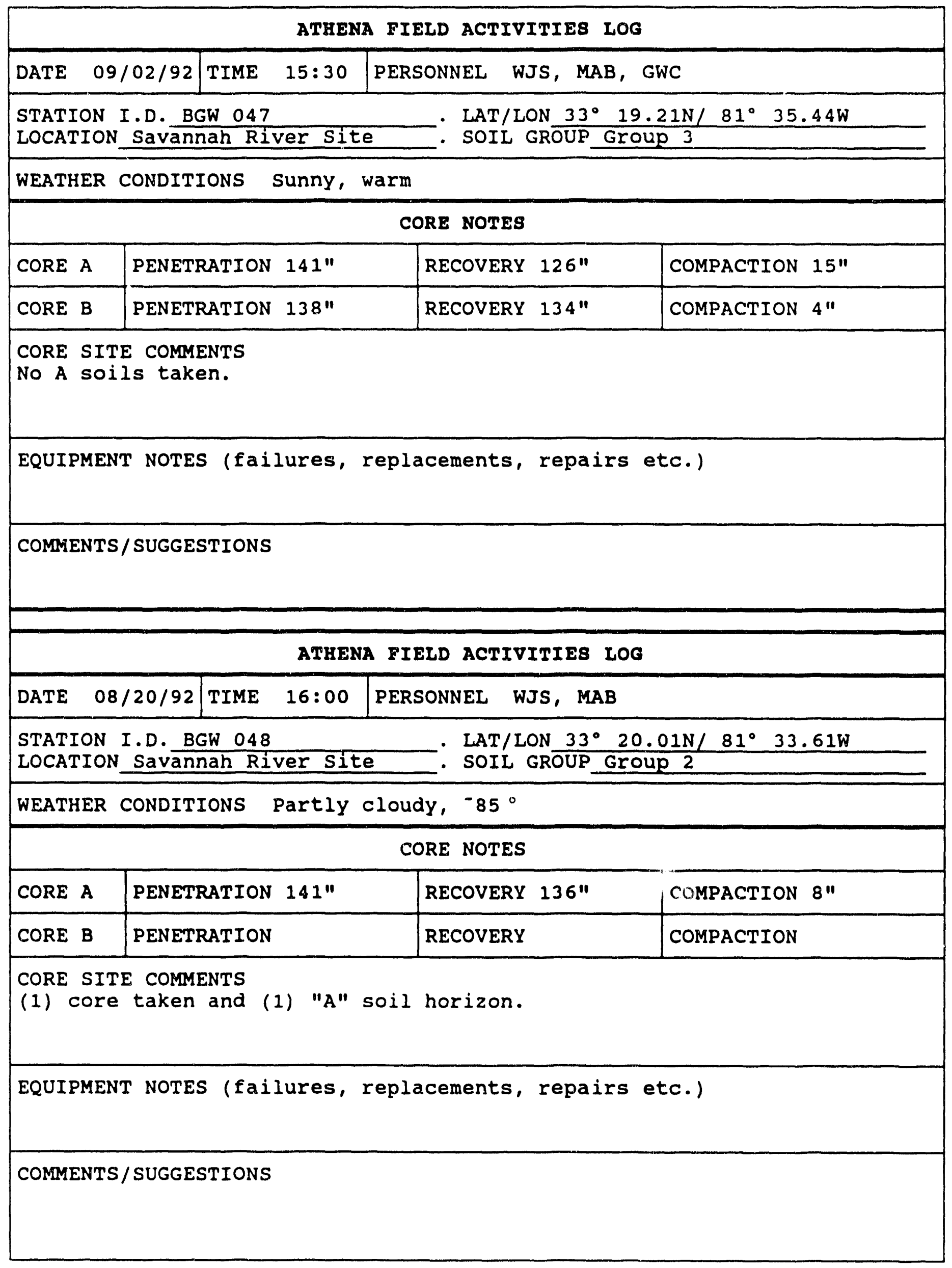




\section{ATHENA FIELD ACTIVITIES LOG}

\begin{tabular}{|ll|l|l|l|}
\hline DATE $08 / 31 / 92$ & TIME 17:00 & PERSONNEL WJS, MAB, GWC \\
\hline
\end{tabular}

STATION I.D. BGW 049 - LAT/LON $33^{\circ} 15.15 \mathrm{~N} / 81^{\circ} 36.81 \mathrm{~W}$ LOCATION Savannah River Site - SOIL GROUP GrOUP 3

WEATHER CONDITIONS SUNnY, warm

CORE NOTES

\begin{tabular}{|l|l|l|l|}
\hline CORE A & PENETRATION 120" & RECOVERY 101" & COMPACTION 18" \\
\hline CORE B & PENETRATION 60" & RECOVERY 50" & COMPACTION 12" \\
\hline
\end{tabular}

CORE SITE COMMENTS

6-8" standing water (swamp), (2) A soils taken.

EQUIPMENT NOTES (failures, replacements, repairs etc.)

COMMENTS/SUGGESTIONS

\section{ATHENA FIELD ACTIVITIES LOG}

\begin{tabular}{|l|l|l|l|l|}
\hline DATE $08 / 31 / 92$ & TIME $14: 30$ & PERSONNEL WJS, MAB \\
\hline
\end{tabular}

STATION I.D. BGW 050

LOCATION Savannah River Site

- LAT/LON $33^{\circ} 14.64 \mathrm{~N} / 81^{\circ} 37.25 \mathrm{~W}$ - SOIL GROUP GrOUP 2

WEATHER CONDITIONS SUNnY, warm, $92^{\circ}$

CORE NOTES

\begin{tabular}{|l|l|l|l|}
\hline CORE A & PENETRATION 56" & RECOVERY 50.5" & COMPACTION 3" \\
\hline CORE B & PENETRATION 72" & RECOVERY 64" & COMPACTION 8" \\
\hline
\end{tabular}

CORE SITE COMMENTS

2 vibracore attempts $(A \& B), 2$ auger attempts $(A \& B)$, refused both times.

EQUIPMENT NOTES (failures, replacenents, repairs etc.) 


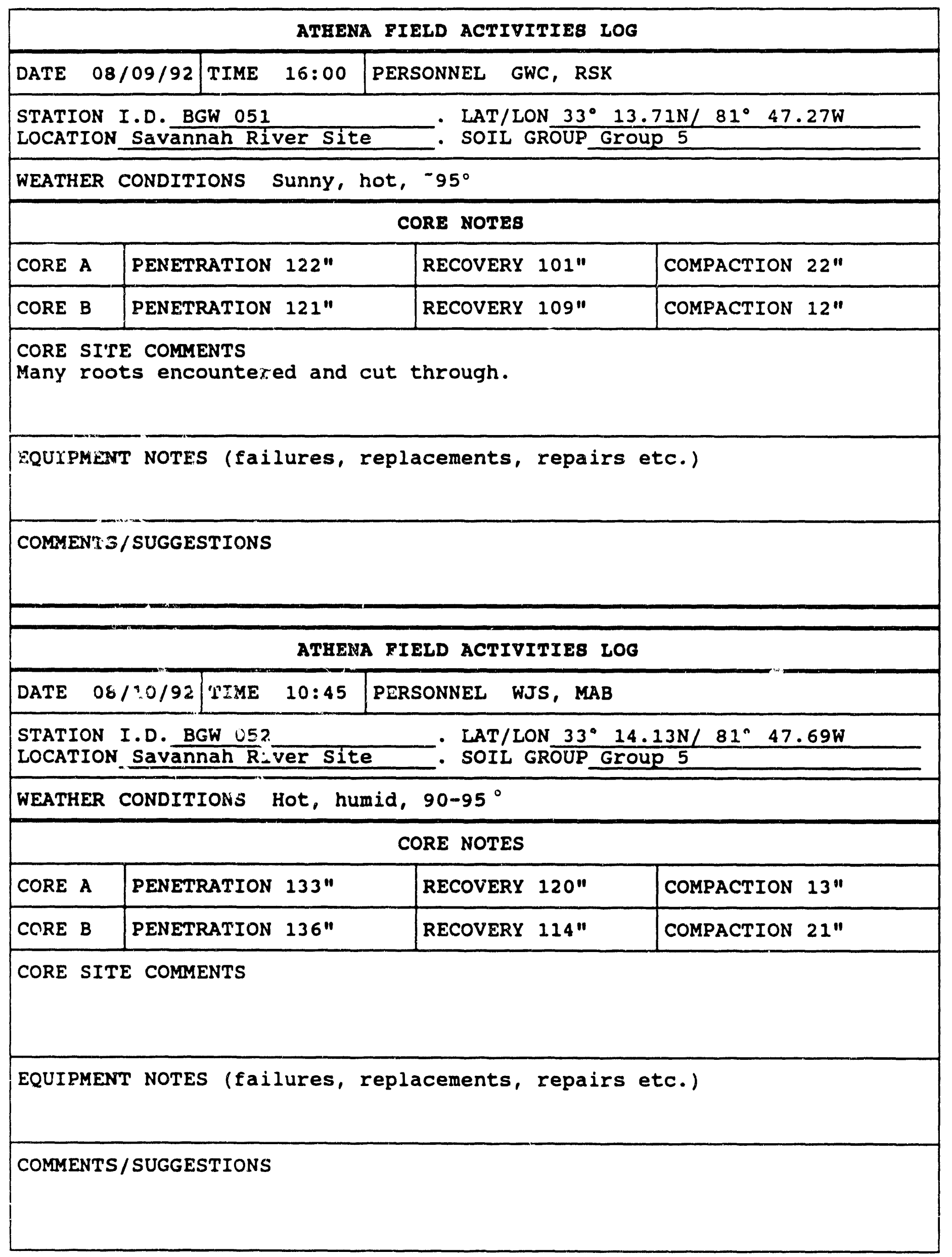




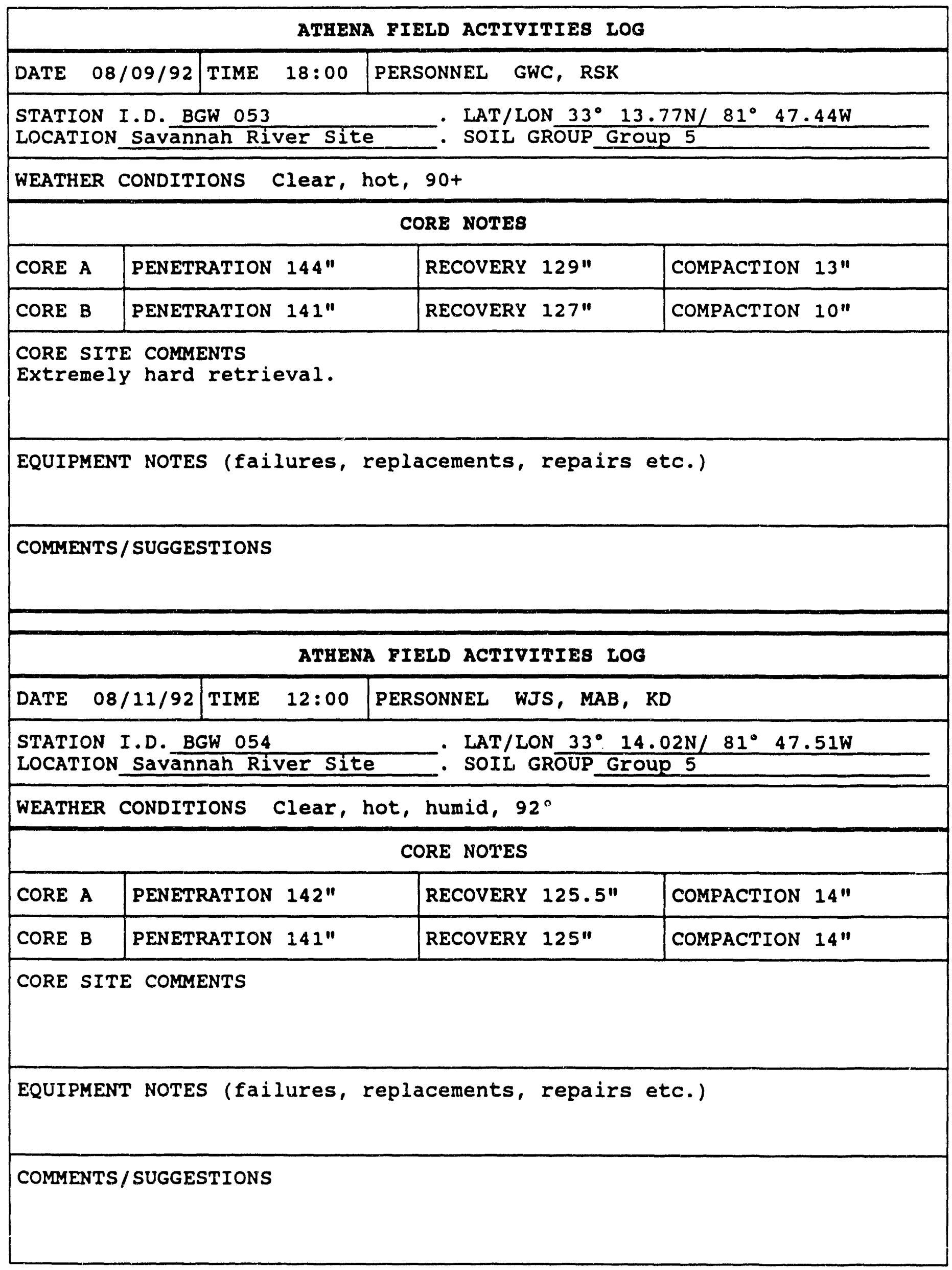




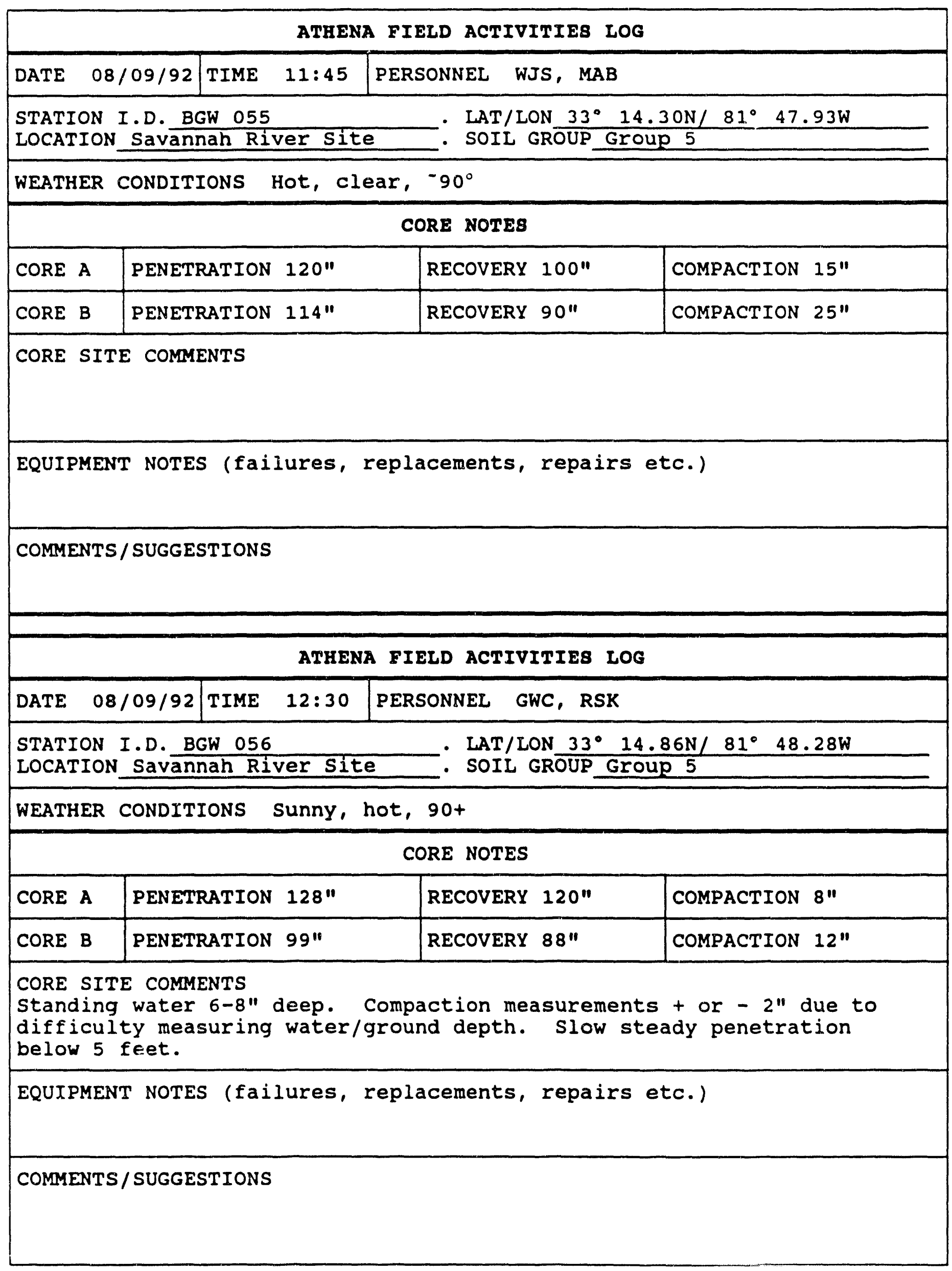




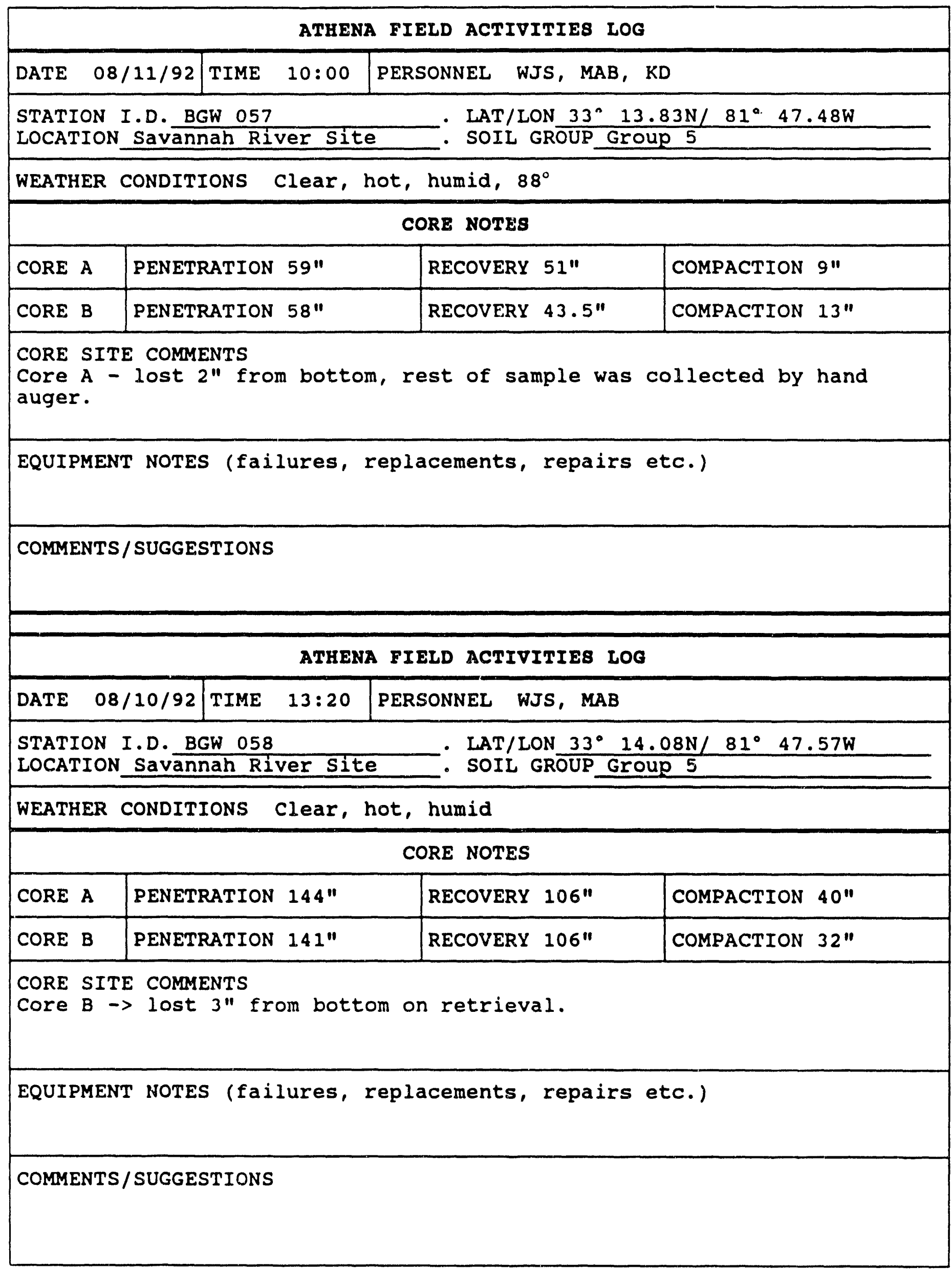


ATHENA FIELD ACTIVITIES LOG

\begin{tabular}{|l|l|l|l|l|}
\hline DATE $08 / 09 / 92$ & TIME $16: 45$ & PERSONNEL WJS, MAB \\
\hline
\end{tabular}

STATION I.D. BGW 059

- LAT/LON $33^{\circ} 14.17 \mathrm{~N} / 81^{\circ} 47.71 \mathrm{~W}$

LOCATION Savannah River Site

- SOIL GROUP GrOUP 5

WEATHER CONDITIONS Hot, clear, humid, 90+

CORE NOTES

\begin{tabular}{|l|l|l|l|}
\hline CORE A & PENETRATION 131" & RECOVERY 116" & COMPACTION 14" \\
\hline CORE B & PENETRATION 136" & RECOVERY 121" & COMPACTION 15" \\
\hline
\end{tabular}

CORE SITE COMMENTS

Failed Core B attempt, additional core taken on $8 / 10 / 92$ at $0900 \rightarrow B$ core.

EQUIPMENT NOTES (failures, replacements, repairs etc.)

COMMENTS/SUGGESTIONS

ATHENA FIELD ACTIVITIES LOG

\begin{tabular}{|l|l|l|l|l|}
\hline DATE $08 / 09 / 92$ & TIME $14: 30$ & PERSONNEL WJS, MAB \\
\hline
\end{tabular}

STATION I.D. BGW 060

LOCATION Savannah River Site

- LAT/LON $33^{\circ} 14.27 \mathrm{~N} / 81^{\circ} 47.82 \mathrm{~W}$

- SOIL GROUP GrOUP 5

WEATHER CONDITIONS Hot, clear, $-95^{\circ}$

CORE NOTES

\begin{tabular}{|l|l|l|l|}
\hline CORE A & PENETRATION 133" & RECOVERY 106" & COMPACTION 22" \\
\hline CORE B & PENETRATION 126" & RECOVERY 111" & COMPACTION 15" \\
\hline
\end{tabular}

CORE SITE COMMENTS

EQUIPMENT NOTES (failures, replacements, repairs etc.)

COMMENTS / SUGGESTIONS 


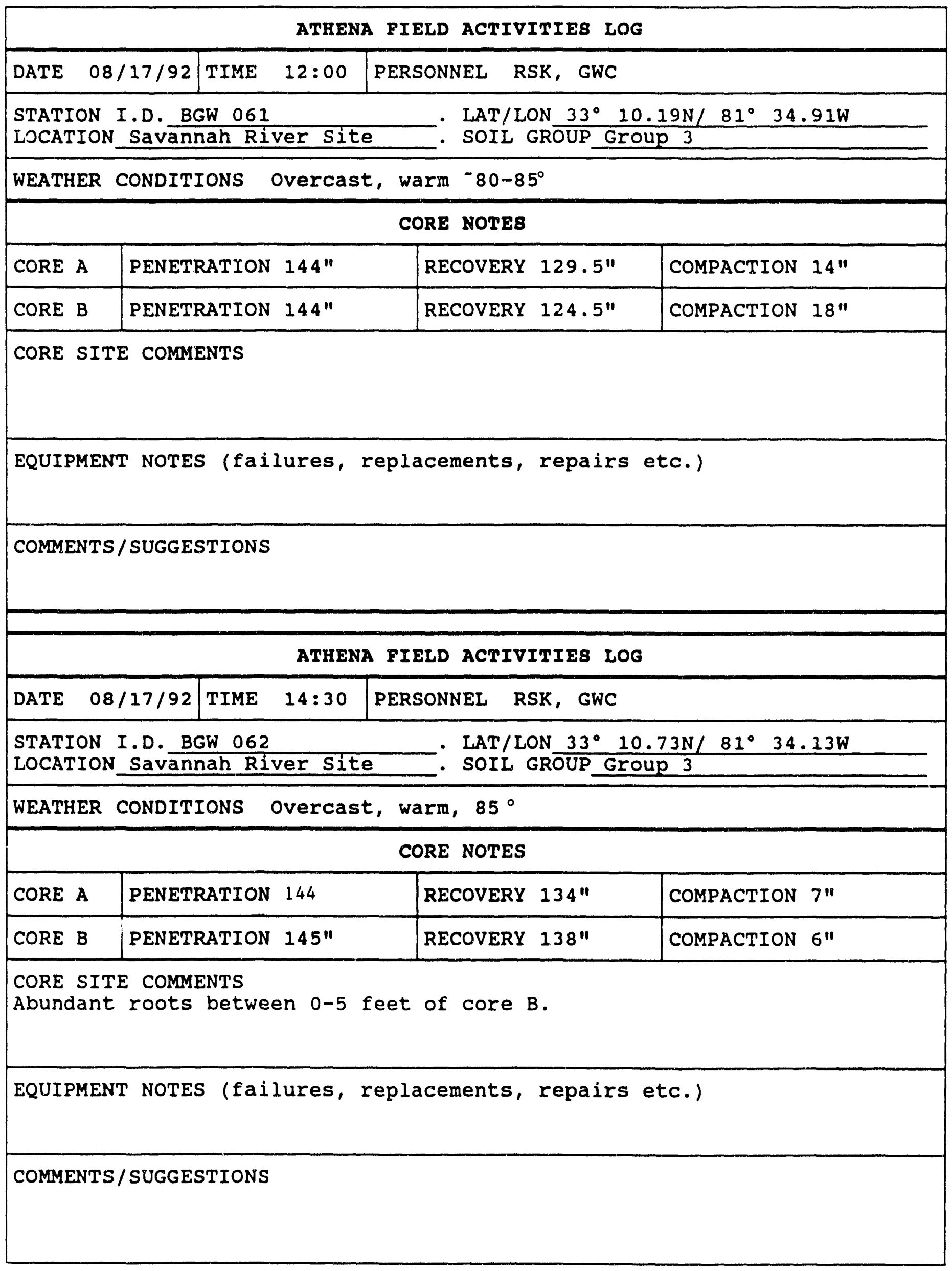




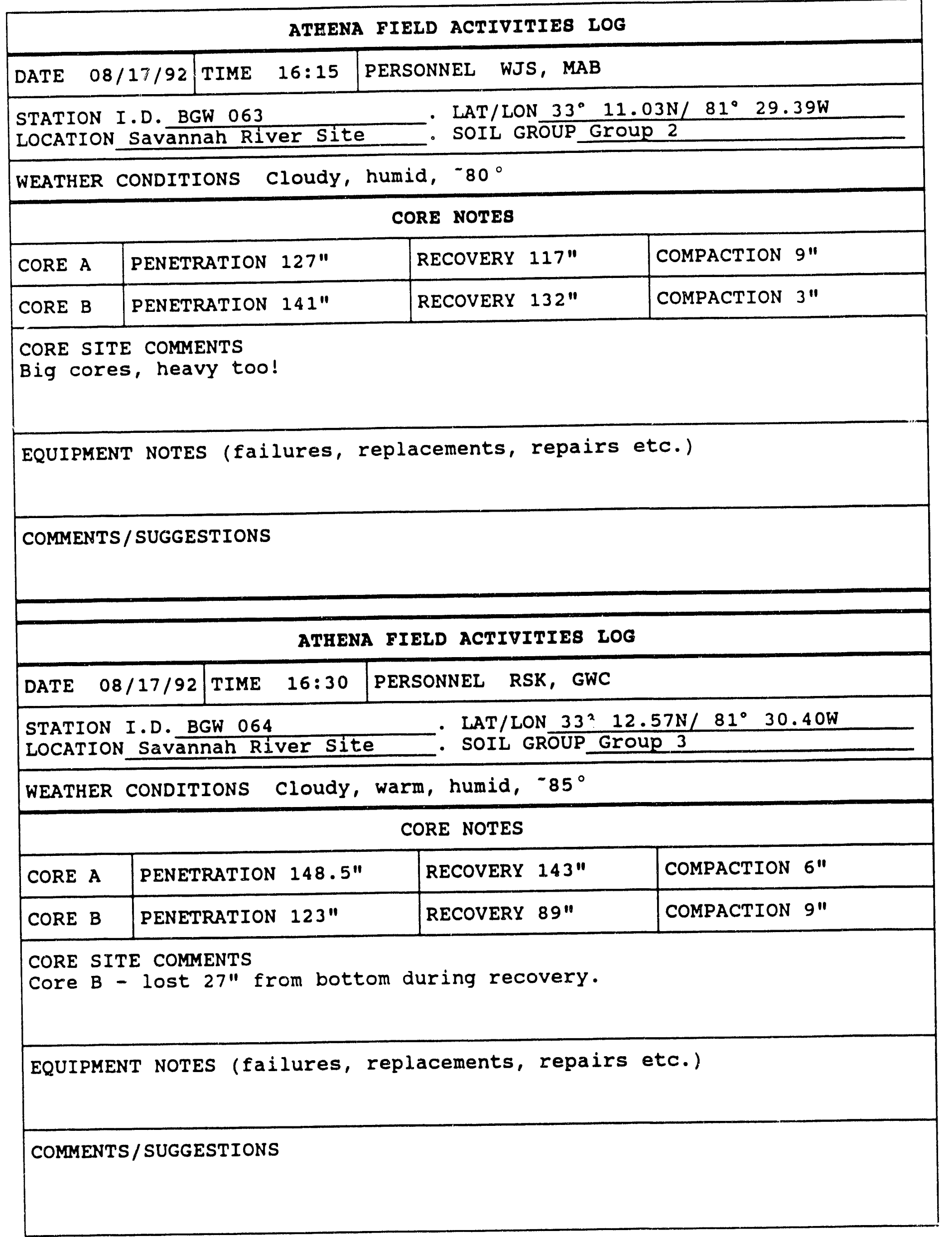




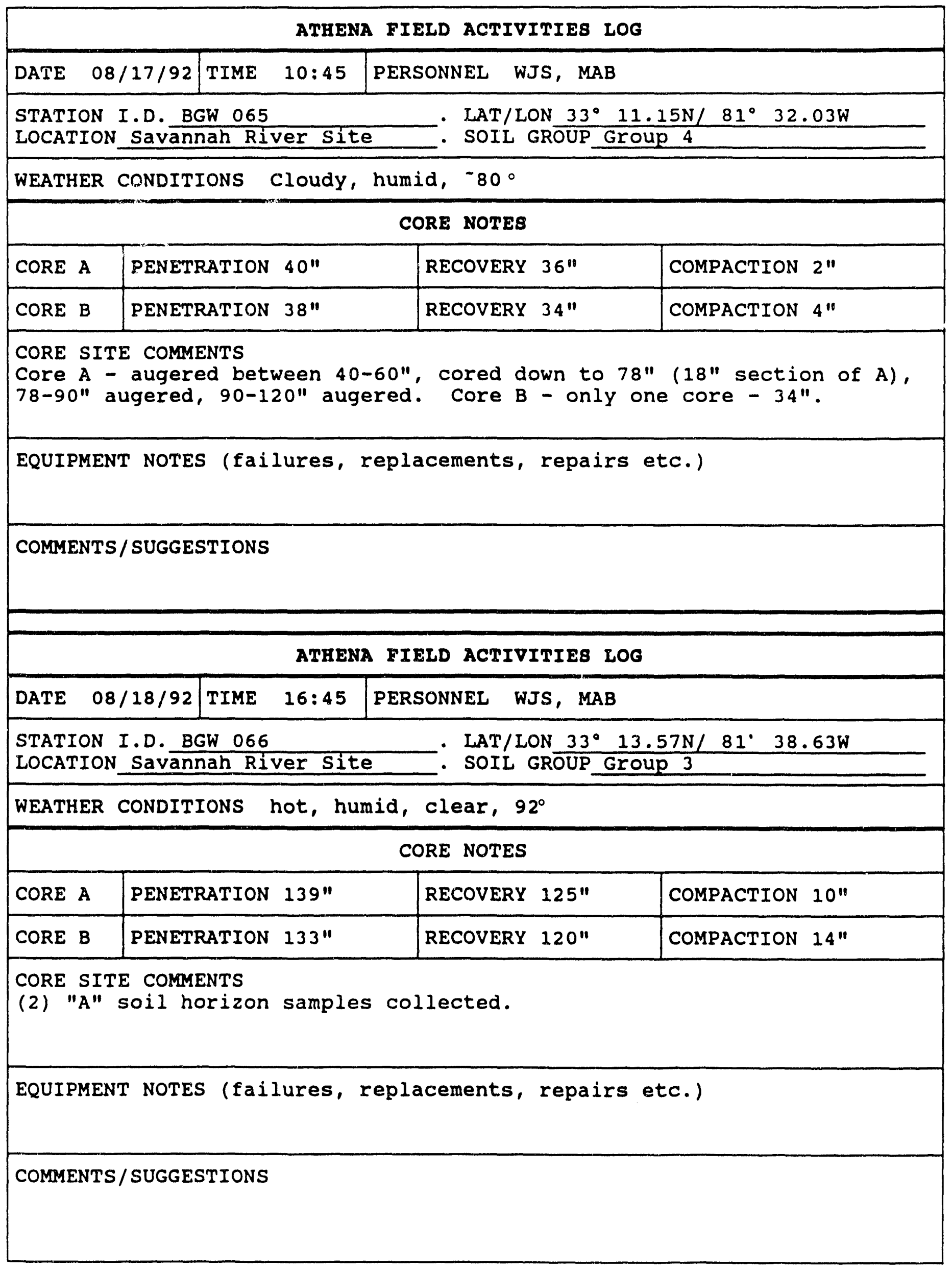




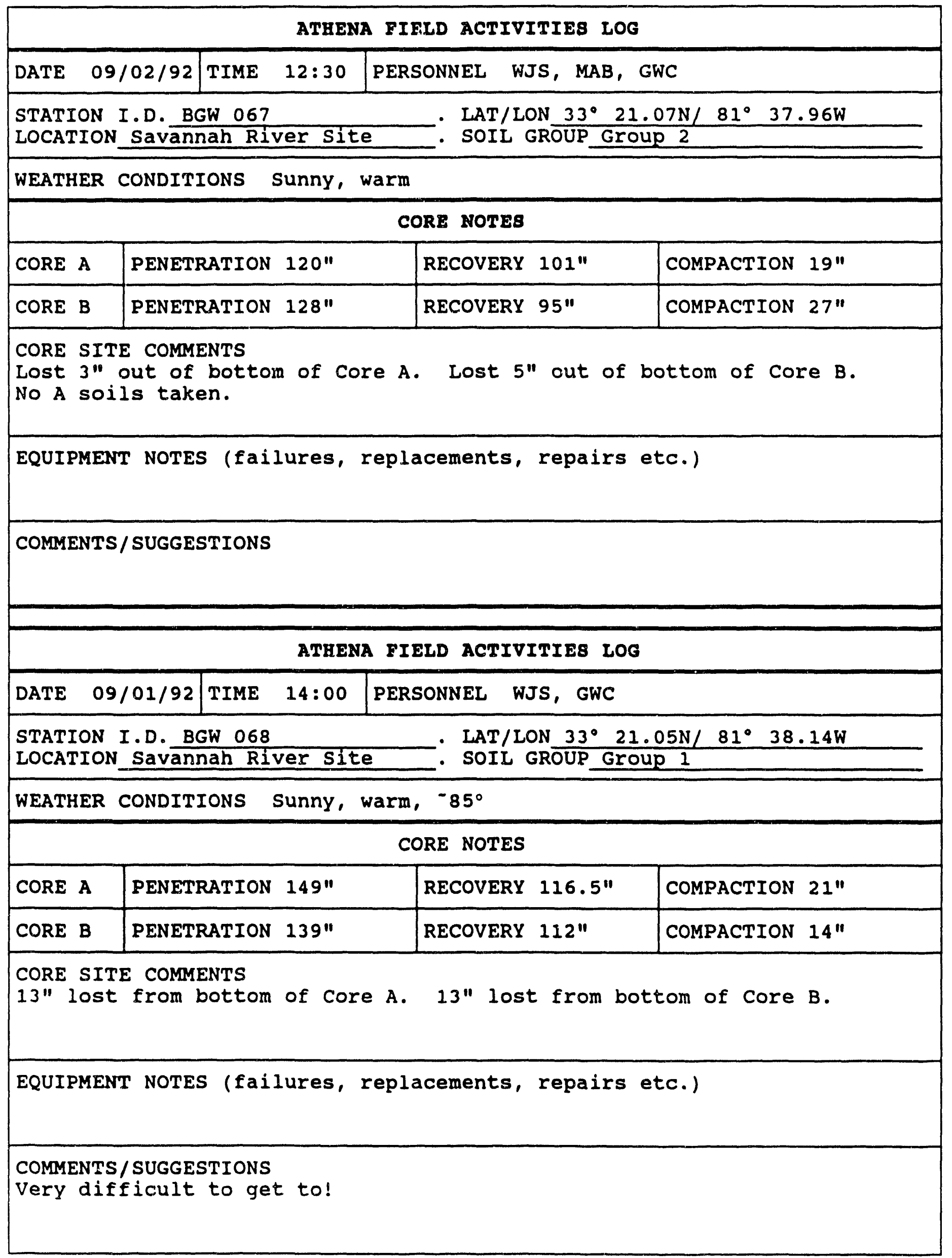




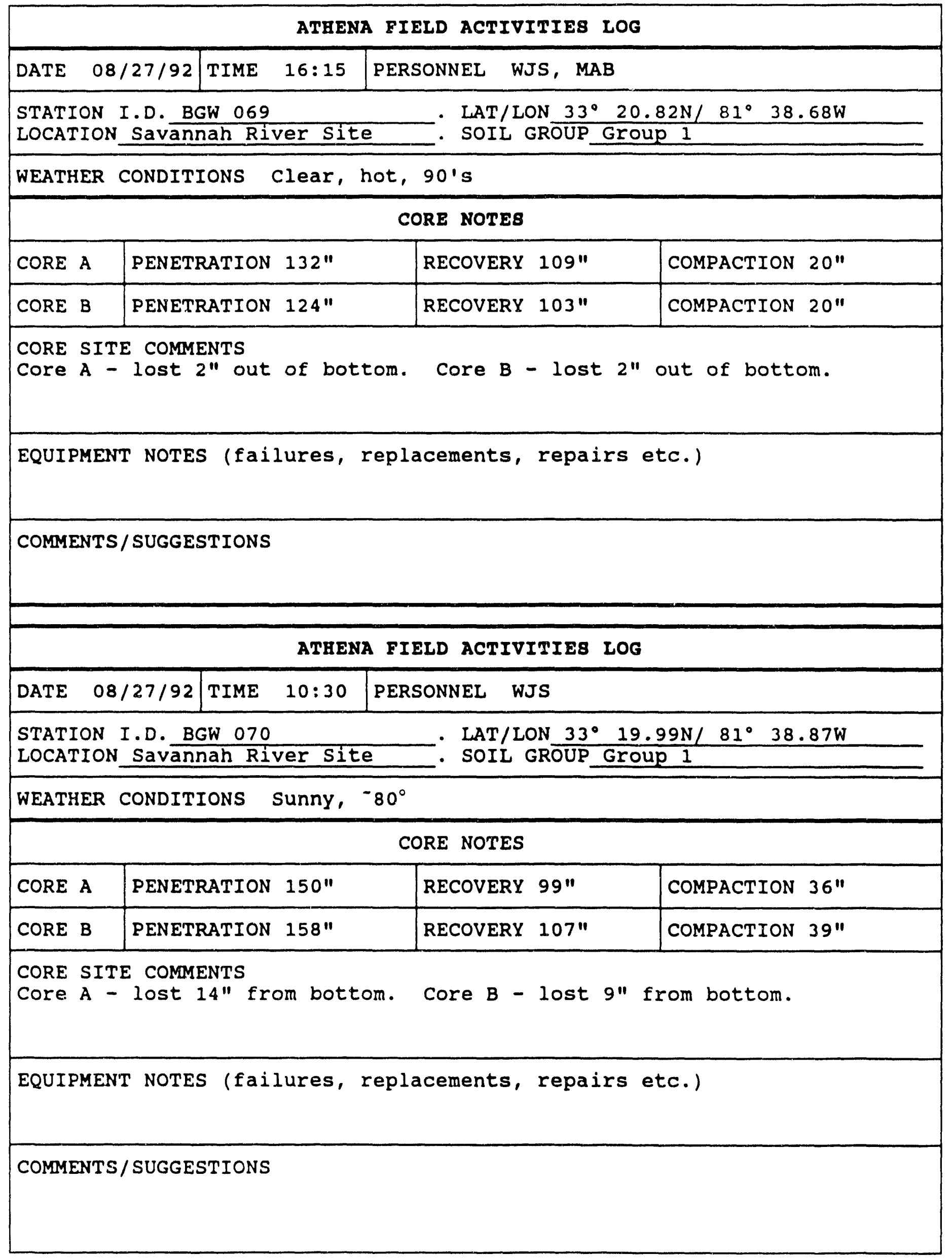




\section{ATHENA FIELD ACTIVITIES LOG}

\begin{tabular}{|ll|l|l|l|}
\hline DATE $08 / 27 / 92$ & TIME 09:00 & PERSONNEL WJS, MAB \\
\hline
\end{tabular}

STATION I.D. BGW 071

- LAT/LON $33^{\circ} 20.02 \mathrm{~N} / 81^{\circ} 38.82 \mathrm{~W}$

LOCATION Savannah River Site

SOIL GROUP GrOUP 1

WEATHER CONDITIONS SUnnY, $-80^{\circ}$

CORE NOTES

\begin{tabular}{|l|l|l|l}
\hline CORE A & PENETRATION 146" & RECOVERY 120" & COMPACTION 23" \\
\hline CORE B & PENETRATION 156" & RECOVERY 125" & COMPACTION 29"
\end{tabular}

CORE SITE COMMENTS

No A soils taken.

EQUIPMENT NOTES (failures, replacements, repairs etc.)

COMMENTS/SUGGESTIONS

ATHENA FIELD ACTIVITIES LOG

\begin{tabular}{|l|l|l|l|l} 
DATE & $08 / 27 / 92$ & TIME $12: 30$ & PERSONNEL WJS, MAB
\end{tabular}

STATION I.D. BGW 072

- LAT/LON $33^{\circ} 20.10 \mathrm{~N} / 81^{\circ} 38.86 \mathrm{~W}$

LOCATION Savannah River Site - SOIL GROUP GrOUP 1

WEATHER CONDITIONS SUNnY, $-85^{\circ}$

CORE NOTES

\begin{tabular}{|l|l|l|l|}
\hline CORE A & PENETRATION 146" & RECOVERY 121" & COMPACTION 24" \\
\hline CORE B & PENETRATION 150" & RECOVERY 132" & COMPACTION 19" \\
\hline
\end{tabular}

CORE SITE COMMENTS

EQUIPMENT NOTES (failures, replacements, repairs etc.)

COMMENTS/SUGGESTIONS 


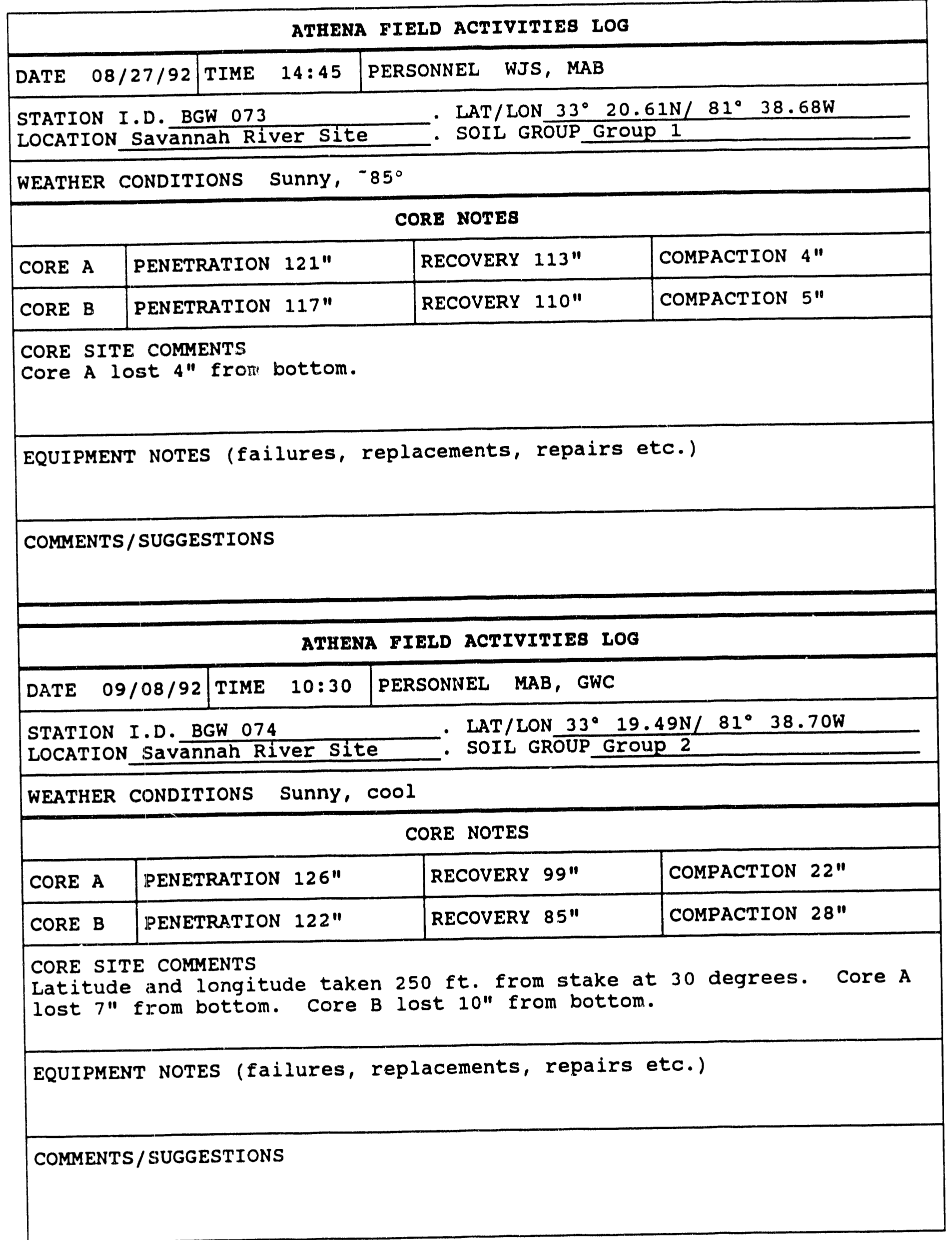




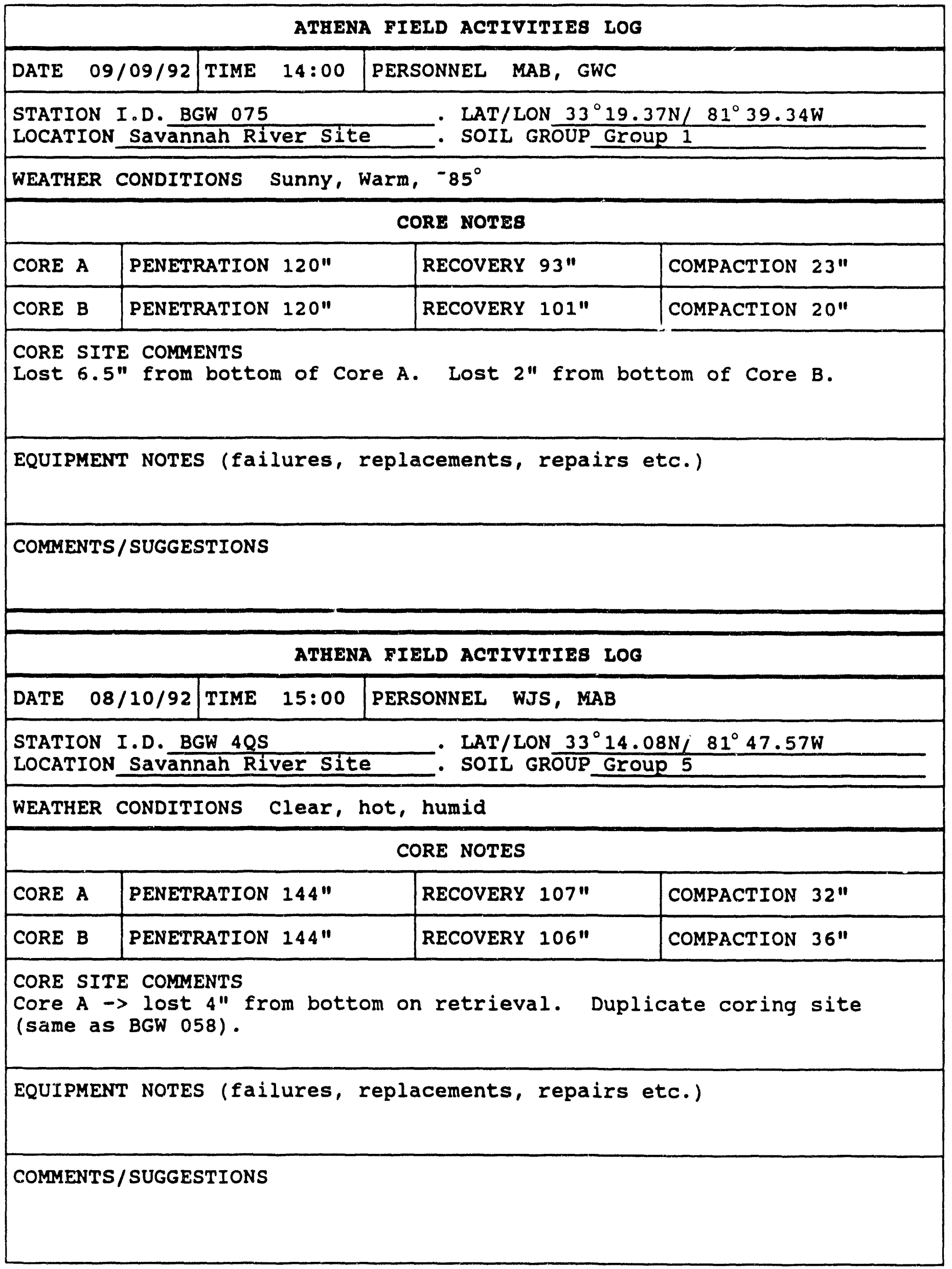




\begin{tabular}{|c|c|c|}
\hline \multicolumn{3}{|c|}{ ATHENA FIELD ACTIVITIES LOG } \\
\hline \multicolumn{3}{|c|}{\begin{tabular}{ll|ll|ll} 
DATE & $08 / 18 / 92$ & TIME & $17: 30$ & PERSONNEL WJS, MAB \\
\end{tabular}} \\
\hline \multicolumn{3}{|c|}{$\begin{array}{ll}\text { STATION I.D. BGW 5QS } & \text { LAT/LON } 33^{\circ} 13.57 \mathrm{~N} / 81^{\circ} 38.63 \mathrm{~W} \\
\text { LOCATION Savannah River Site } & \text { SOIL GROUP Group } 3\end{array}$} \\
\hline \multicolumn{3}{|c|}{ WEATHER CONDITIONS HOt, humid, clear } \\
\hline \multicolumn{3}{|c|}{ CORE NOTES } \\
\hline PENETRATION $123 "$ & RECOVERY $112 "$ & COMPACTION $14^{\prime \prime}$ \\
\hline PENETRATION $132 "$ & RECOVERY $112^{\prime \prime}$ & COMPACTION $17^{\prime \prime}$ \\
\hline \multicolumn{3}{|c|}{$\begin{array}{l}\text { CORE SITE COMMENTS } \\
\text { (2) "A" soil horizon samples collected. }\end{array}$} \\
\hline \multicolumn{3}{|c|}{ EQUIPMENT NOTES (failures, replacements, repairs etc.) } \\
\hline \multicolumn{3}{|l|}{ COMMENTS/SUGGESTIONS } \\
\hline \multicolumn{3}{|c|}{ ATHENA FIELD ACTIVITIES LOG } \\
\hline \multicolumn{3}{|c|}{\begin{tabular}{|l|l|l|ll} 
DATE & $08 / 25 / 92$ & TIME & $14: 40$ & PERSONNEL WJS, MAB, GWC \\
\end{tabular}} \\
\hline \multicolumn{3}{|c|}{$\begin{array}{l}\text { STATION I.D. BGW 6QS } \\
\text { LOCATION Savannah River Site }\end{array}$} \\
\hline \multicolumn{3}{|c|}{ WEATHER CONDITIONS Partly cloudy, windy, pleasant } \\
\hline \multicolumn{3}{|c|}{ CORE NOTES } \\
\hline PENETRATION 120" & RECOVERY $120^{\prime \prime}$ & COMPACTION O" \\
\hline PENETRATION & RECOVERY & COMPACTION \\
\hline \multicolumn{3}{|c|}{$\begin{array}{l}\text { CORE SITE COMMENTS } \\
\text { A and B cores were driven with hammer. Auger started at } 20^{\prime \prime} \text {. (2) A } \\
\text { soils taken. }\end{array}$} \\
\hline \multicolumn{3}{|c|}{ EQUIPMENT NOTES (failures, replacements, repairs etc.) } \\
\hline COMMENTS, SUGEESTIONS & & \\
\hline
\end{tabular}




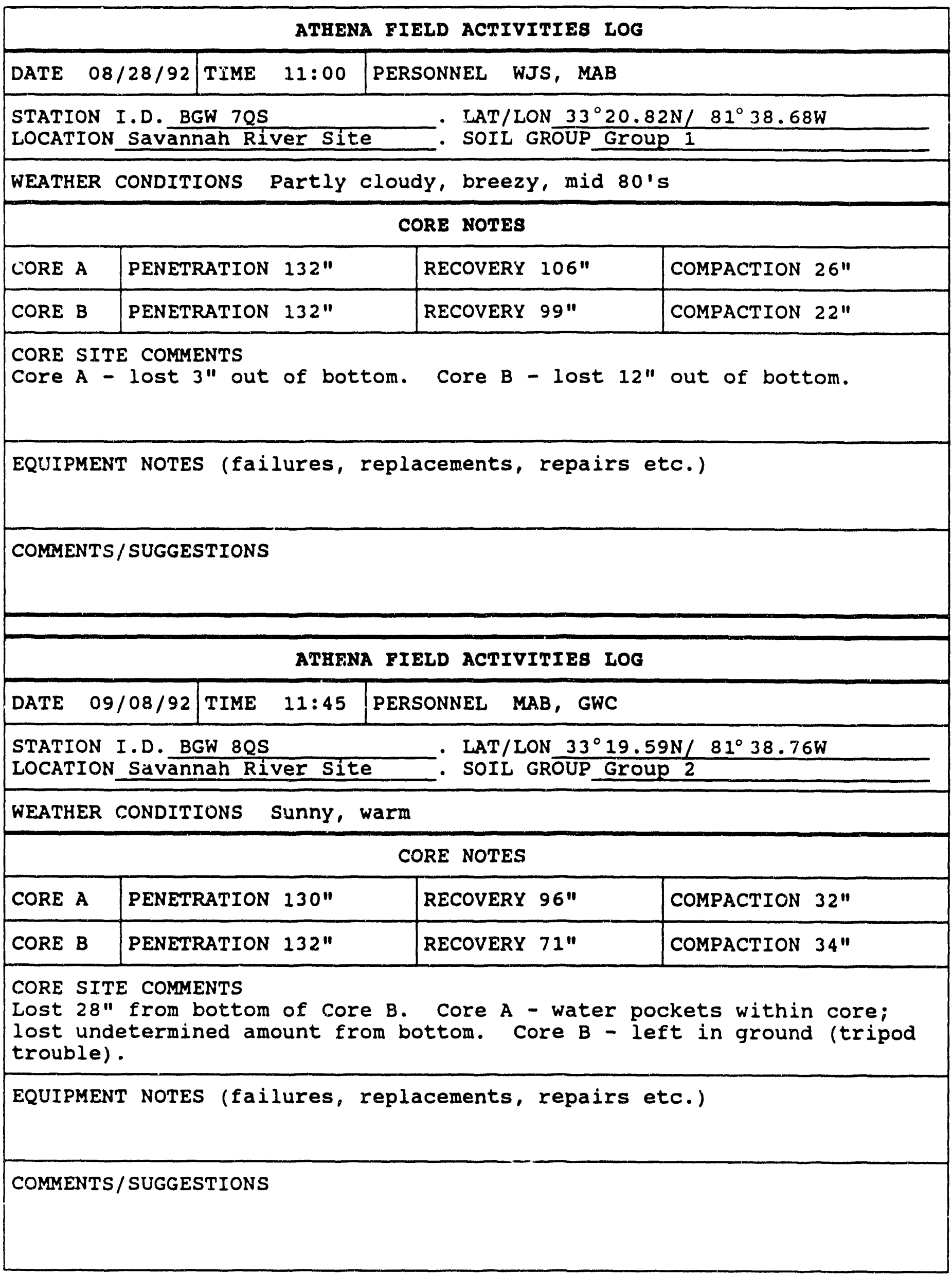


Appendix C

Chain of Custody Forms 


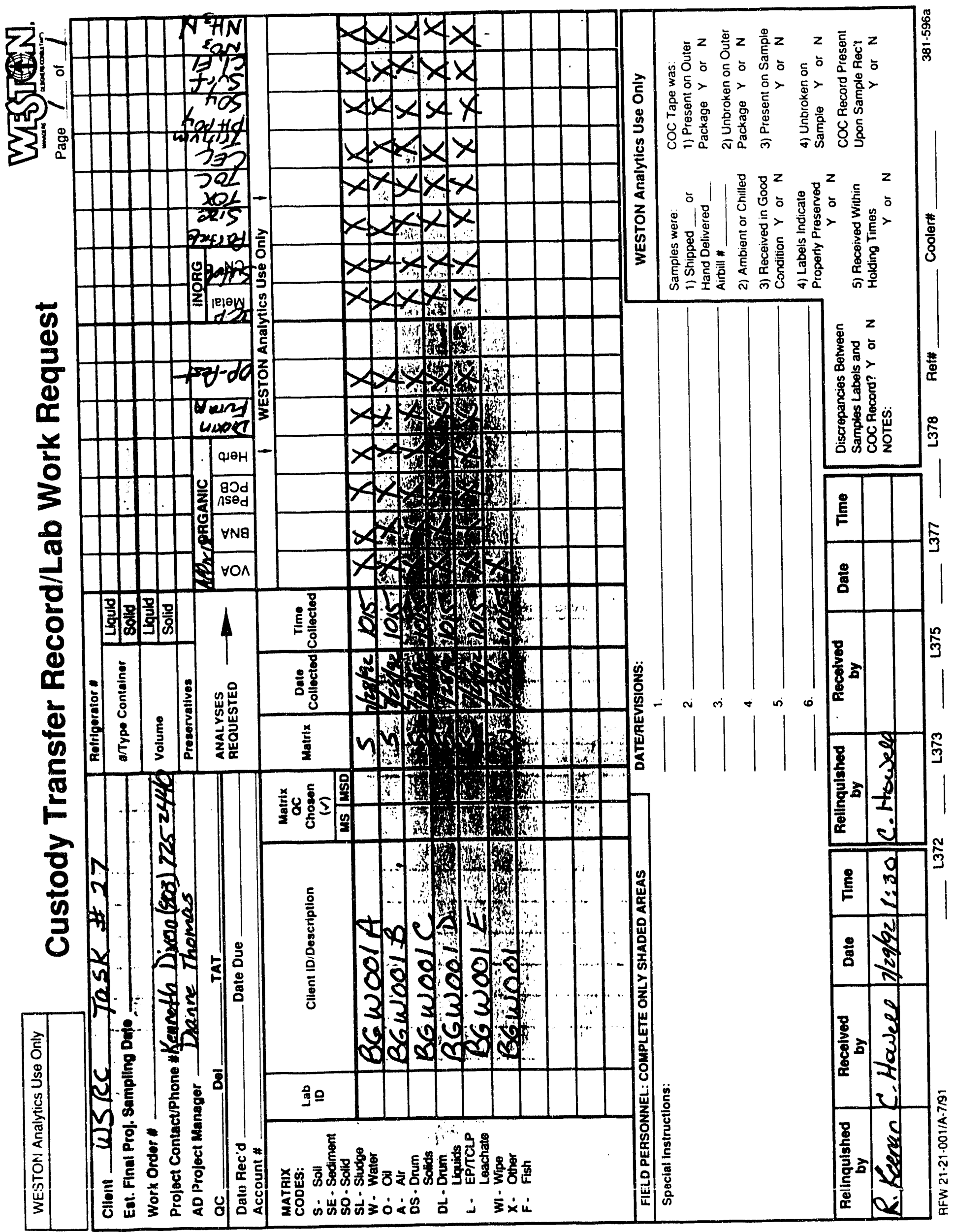




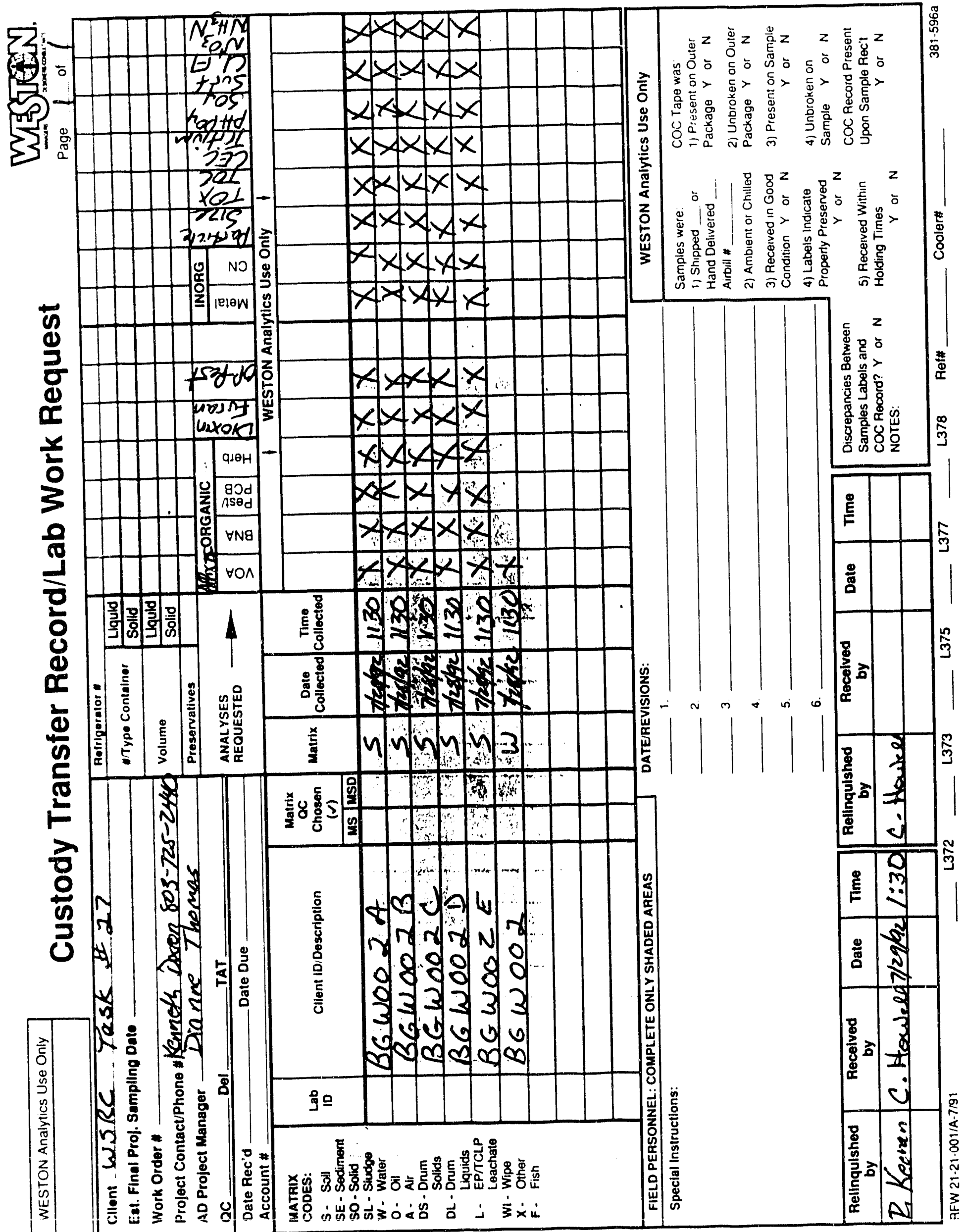




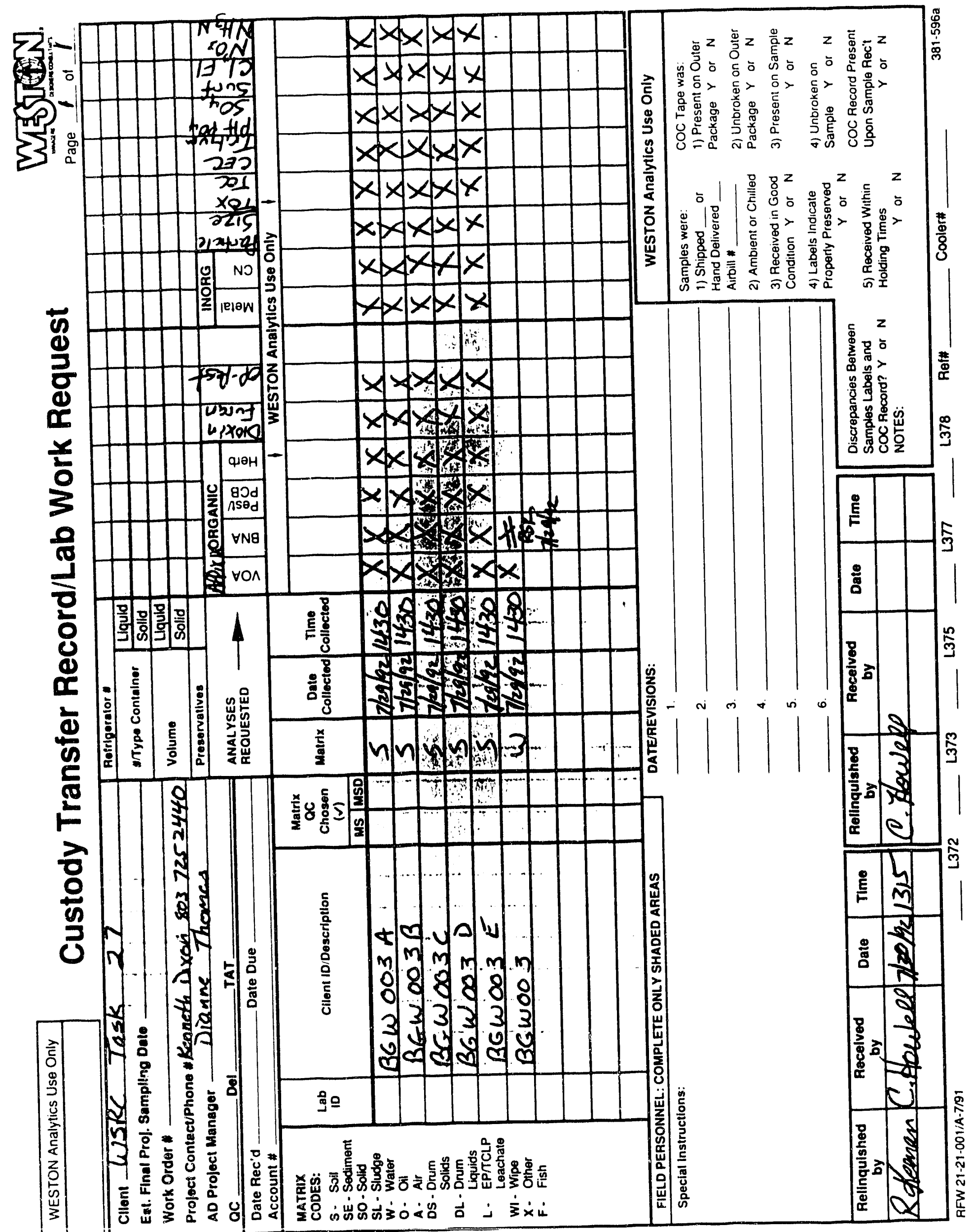




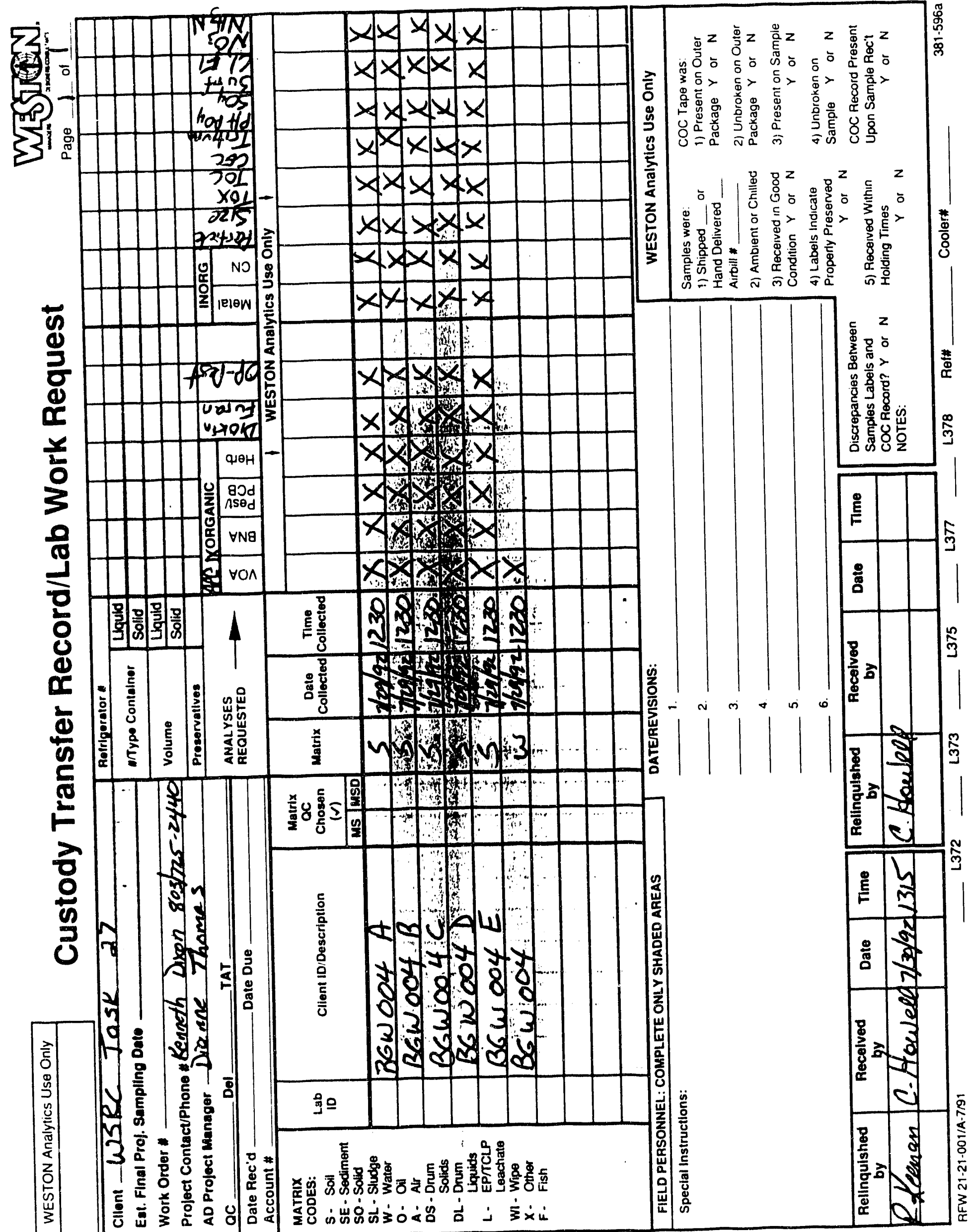




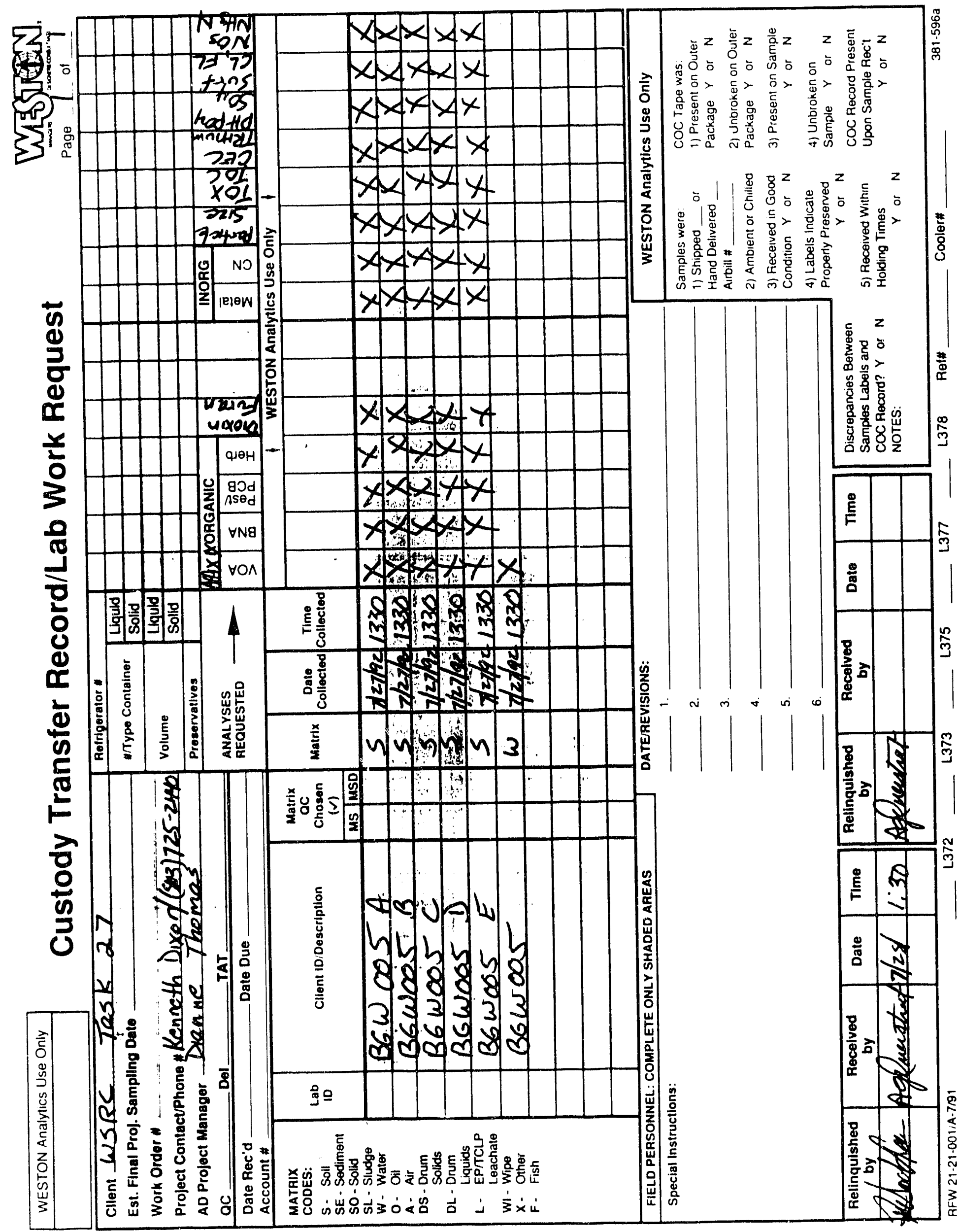




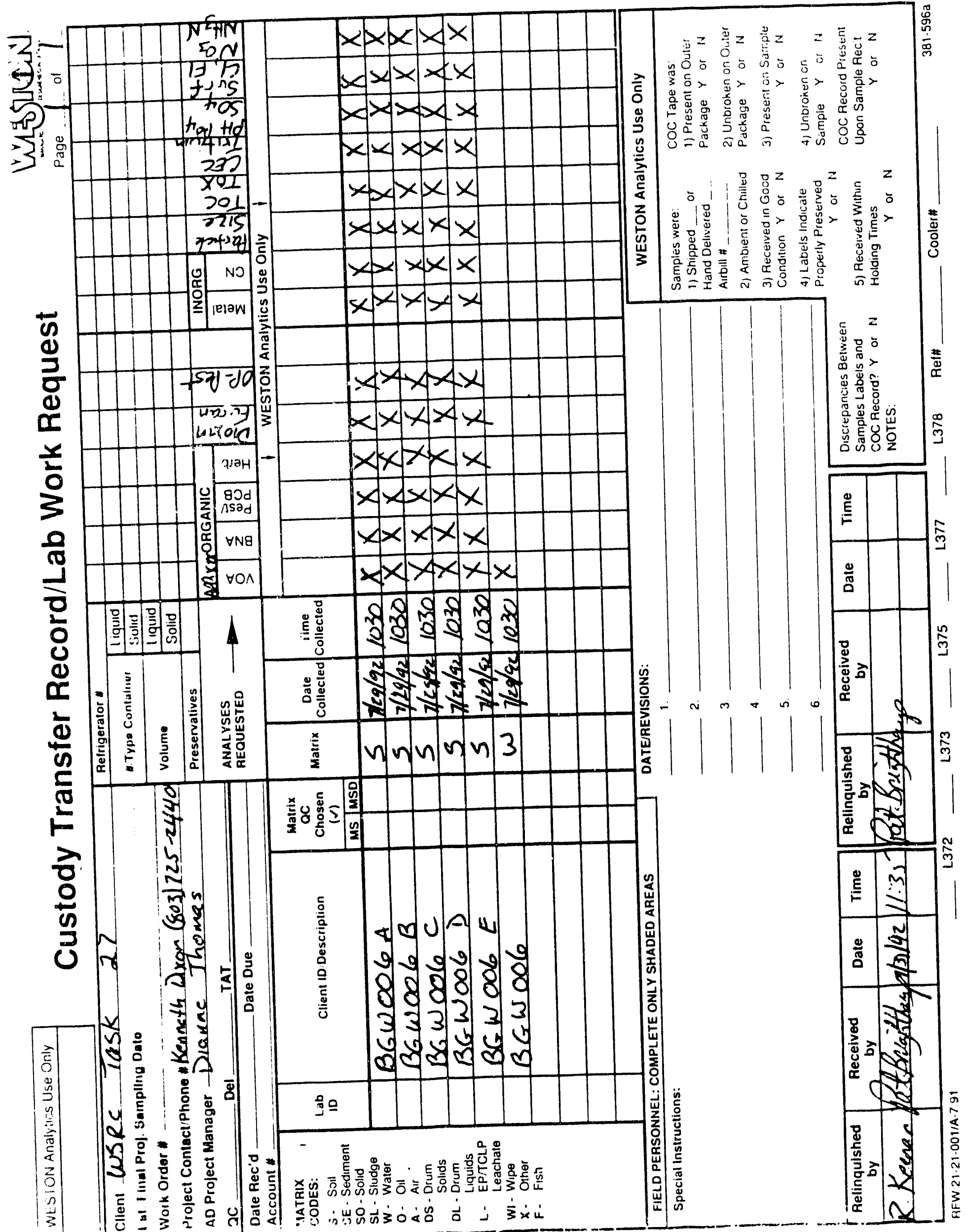




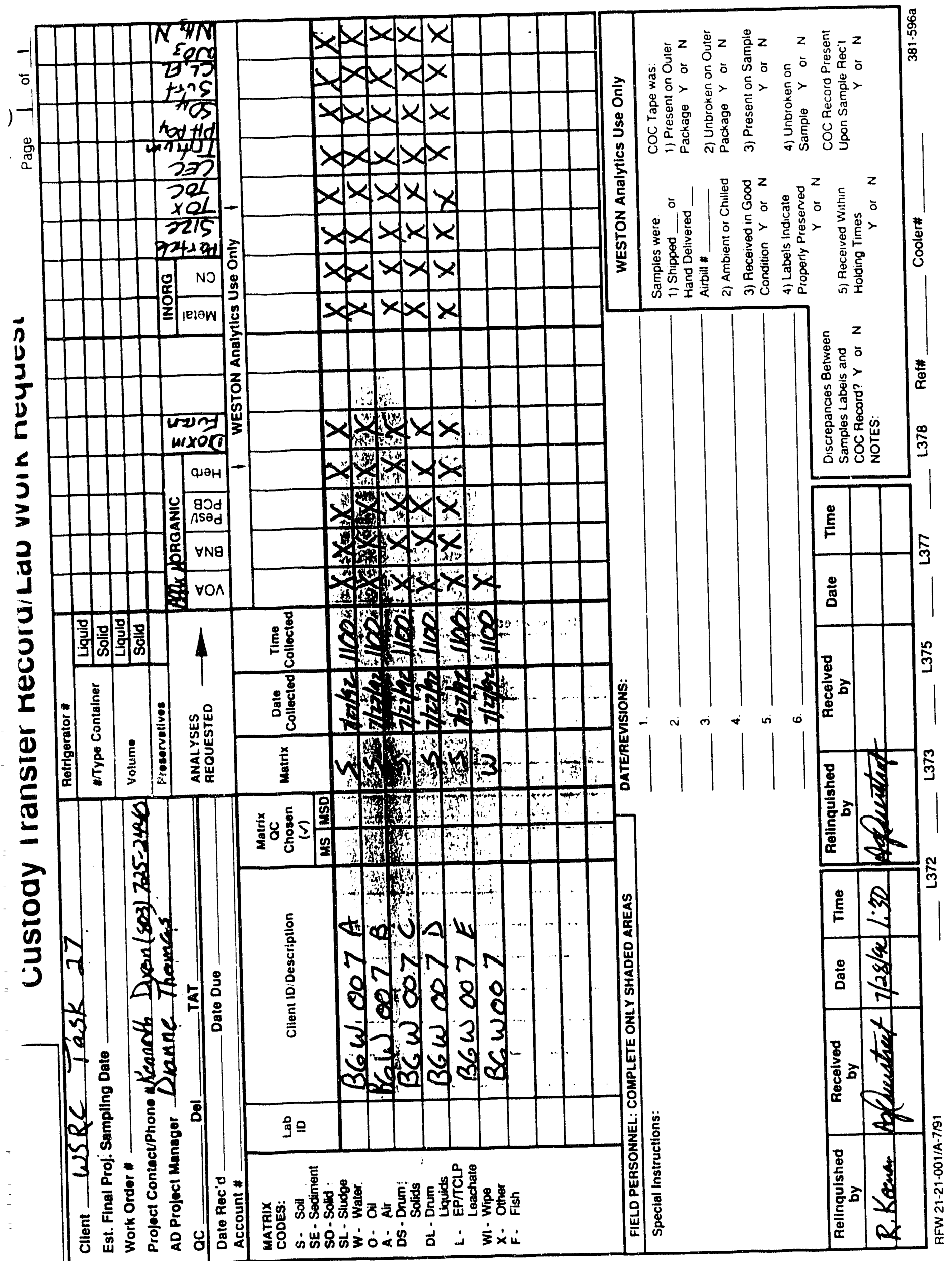




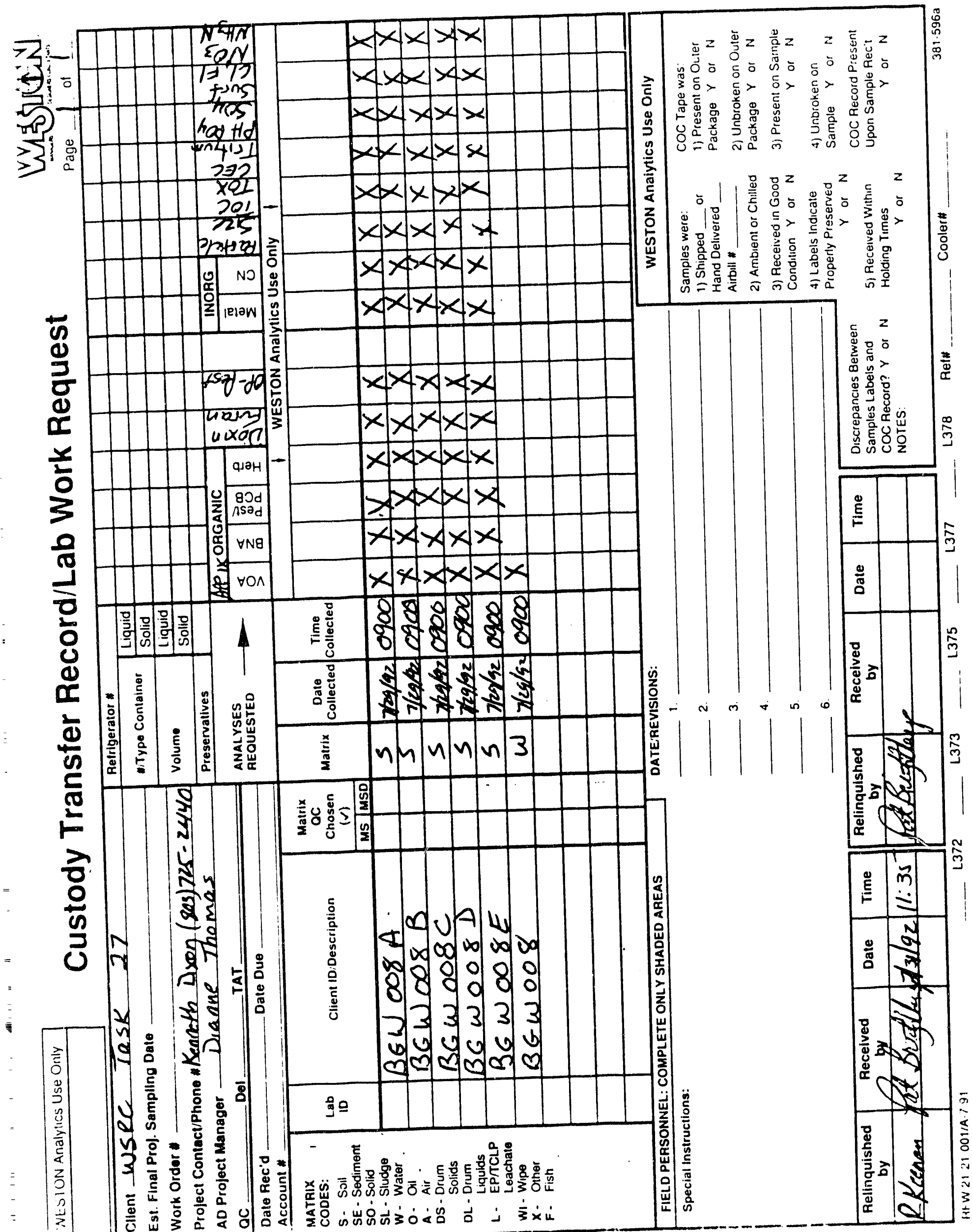




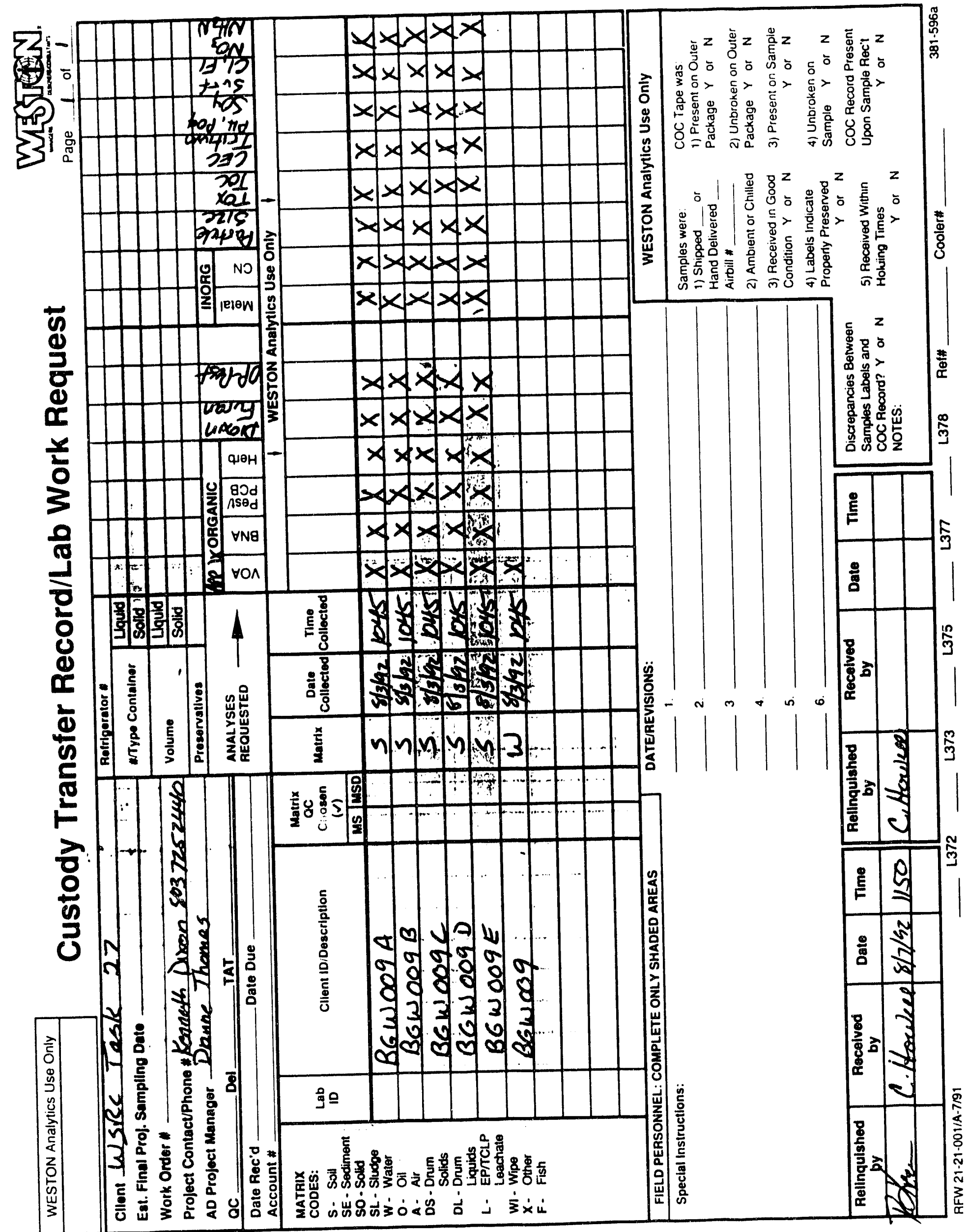




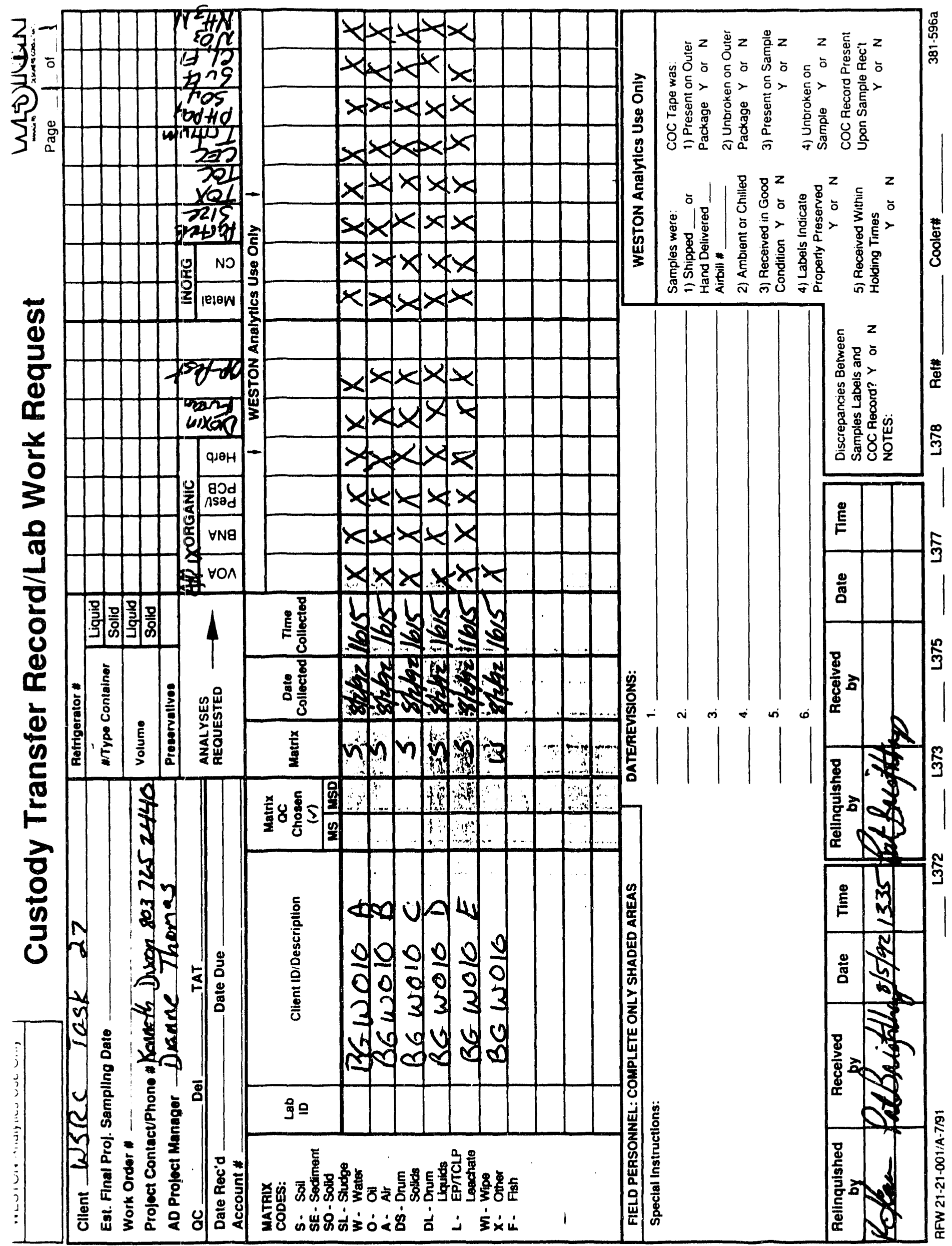




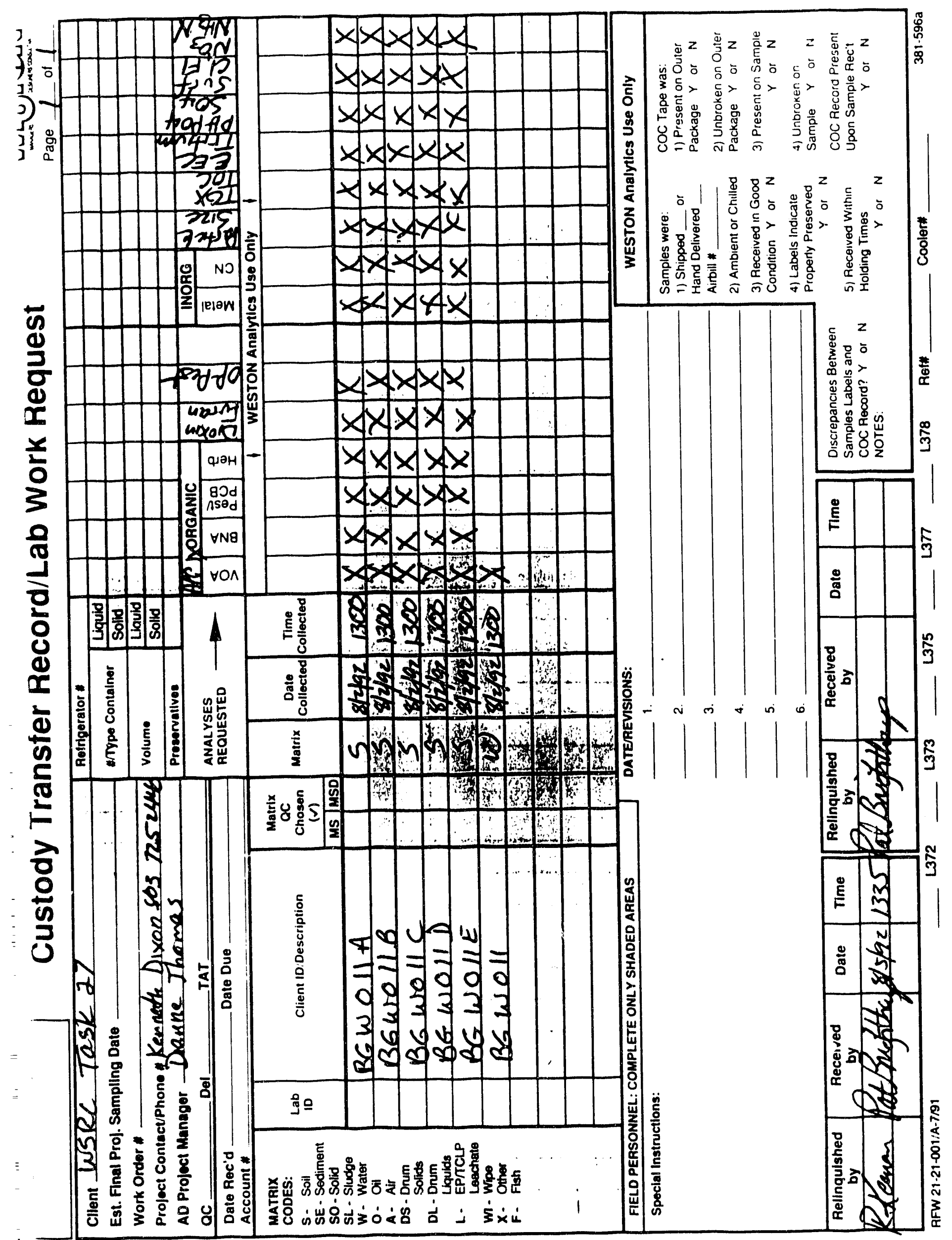




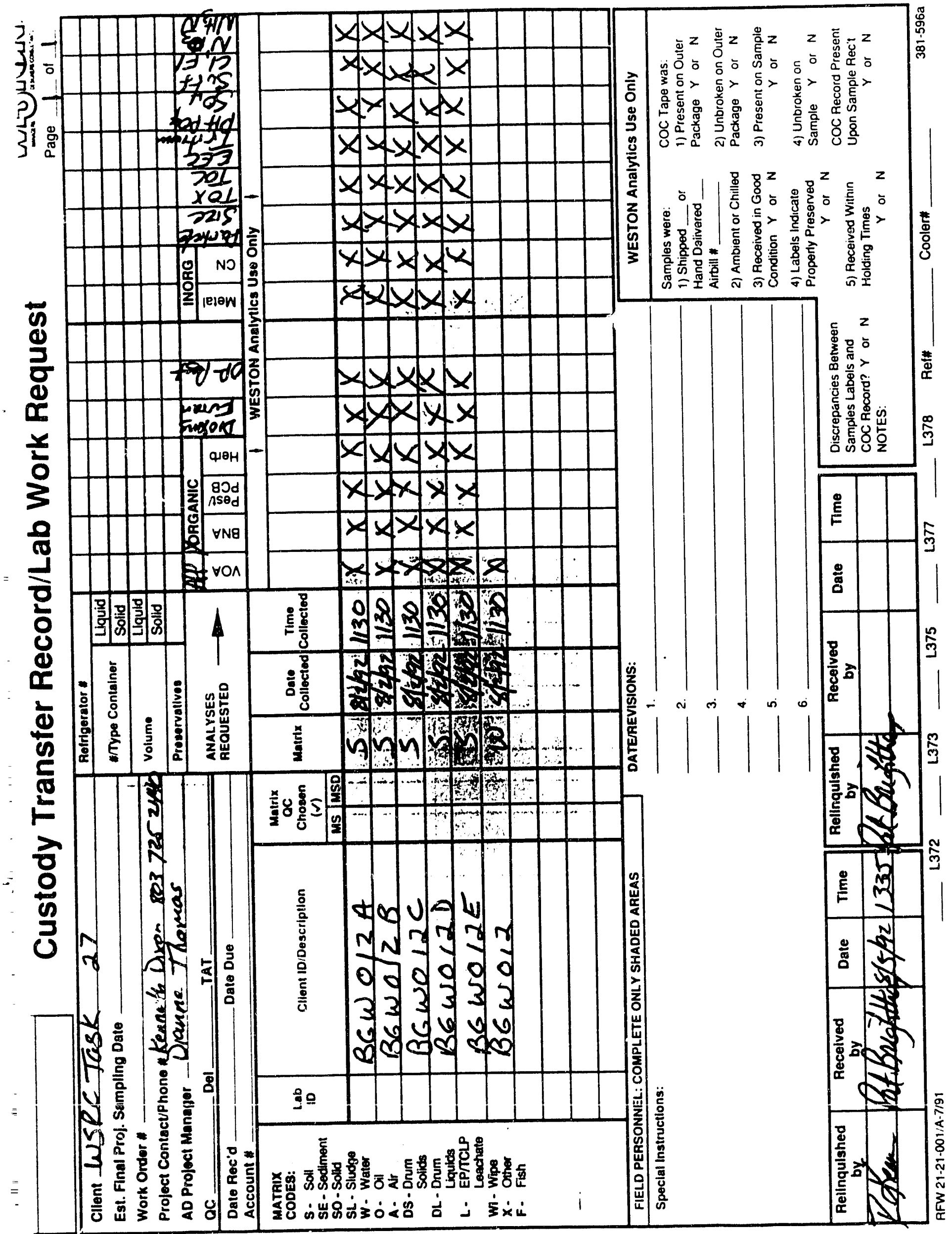




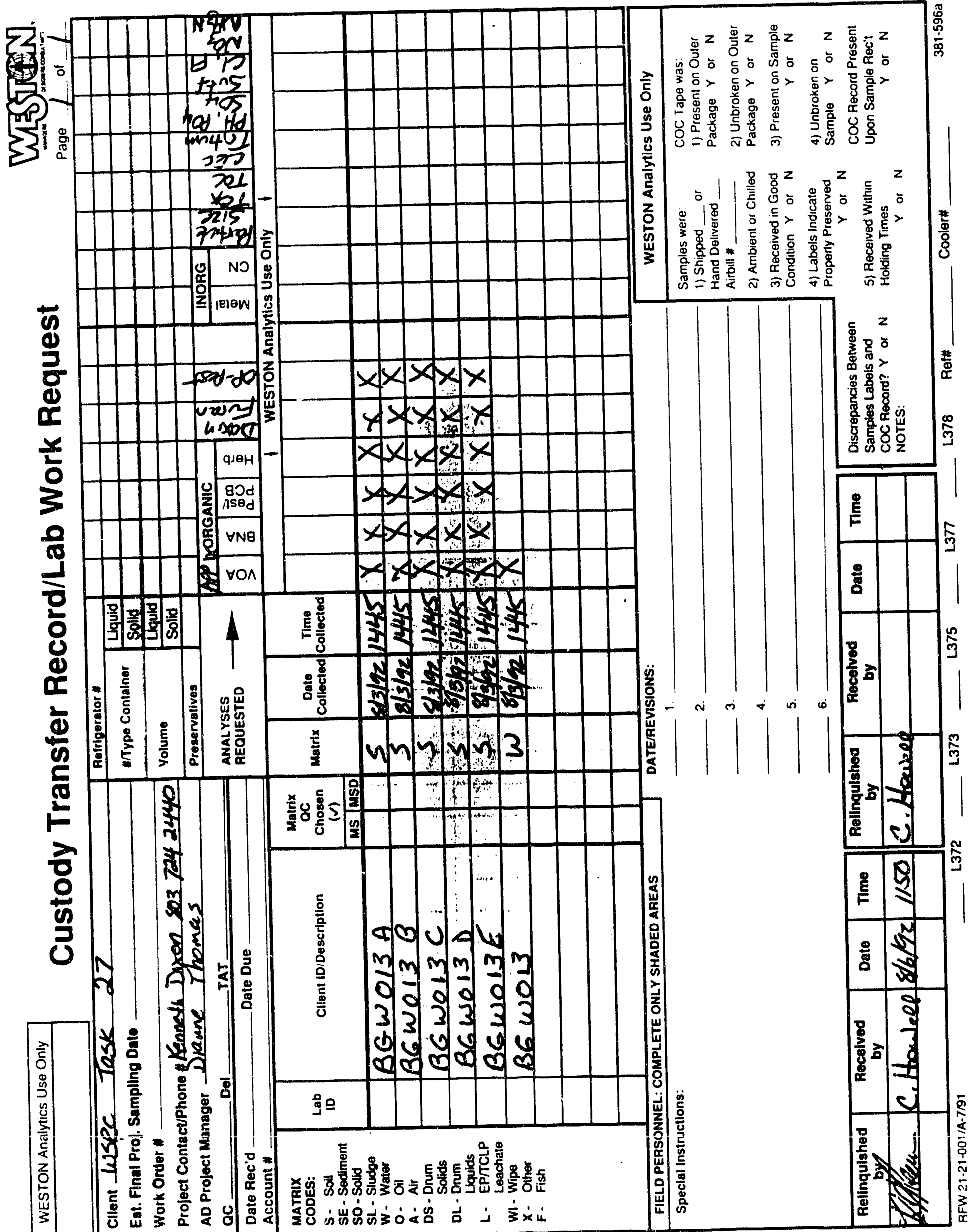




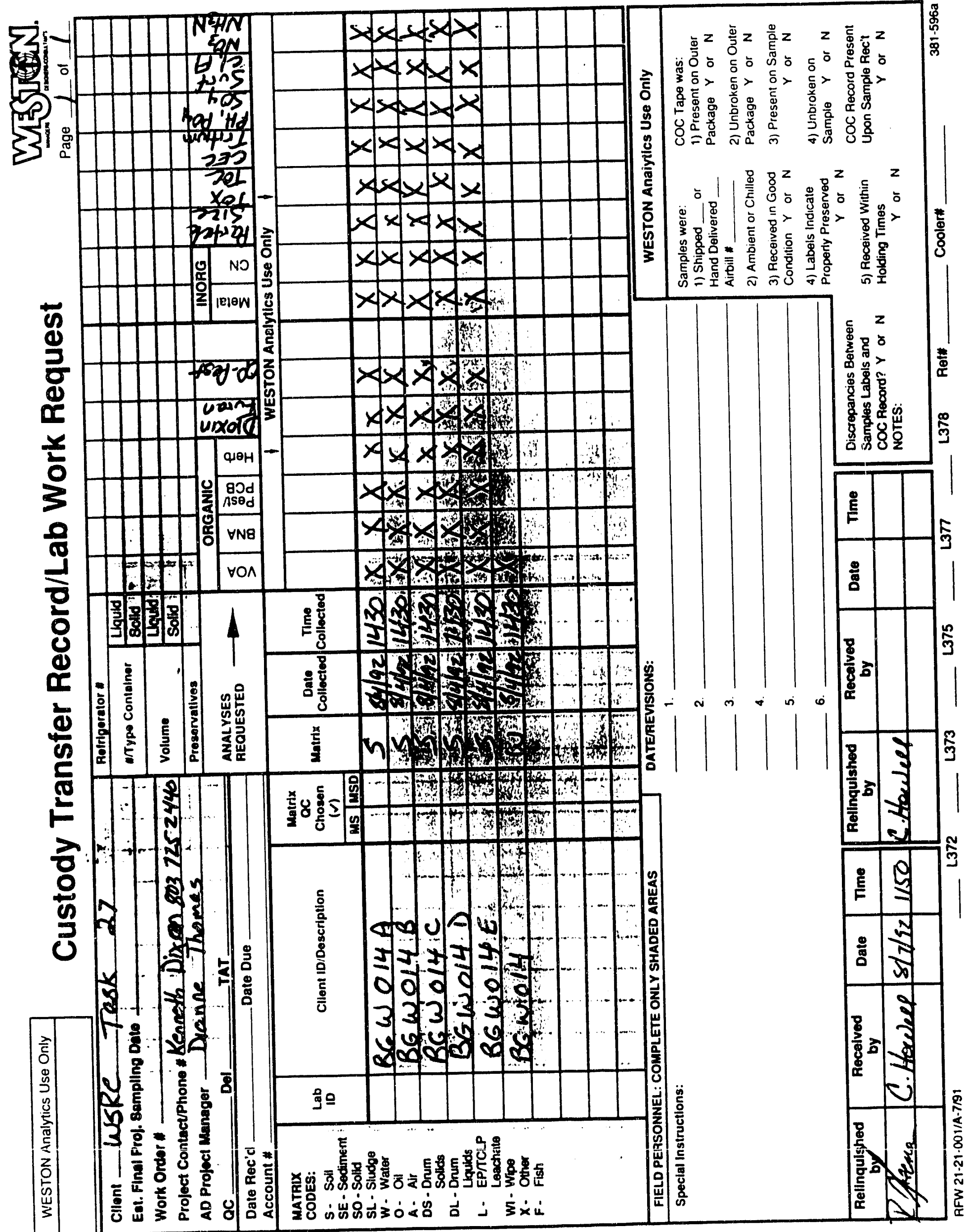




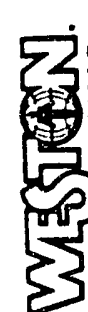

管

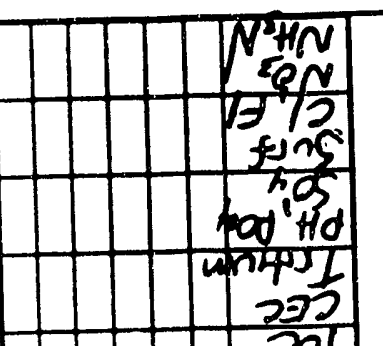

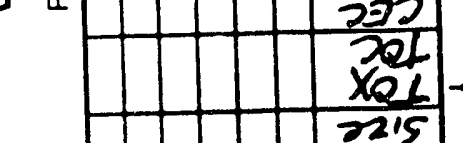

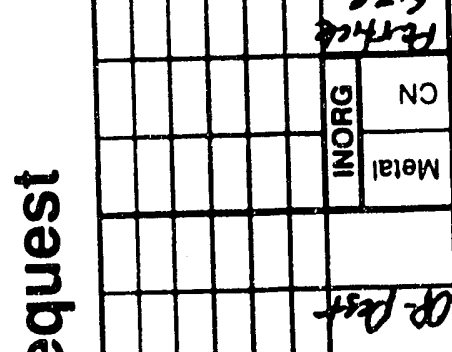

。

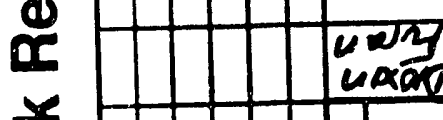

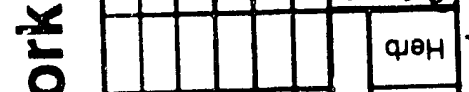

2

8



2

8$$
8
$$

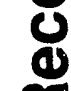

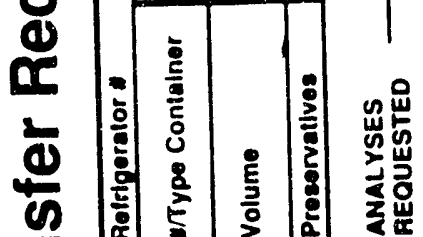

(2)

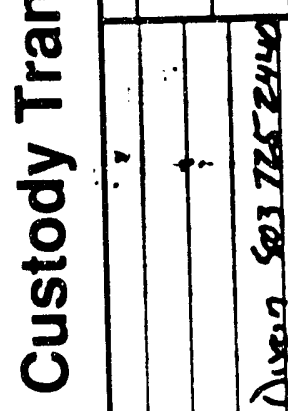

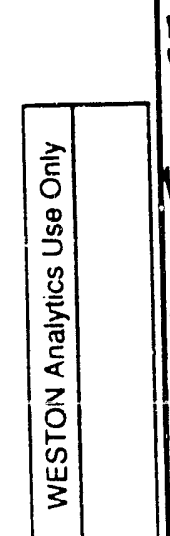

मे :

H

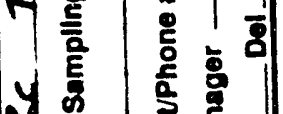

远

ทำ 월

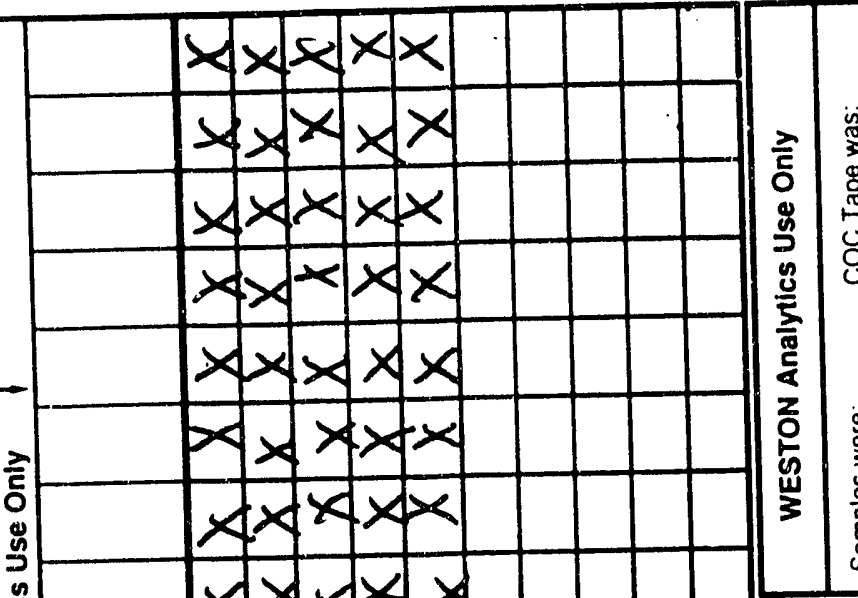

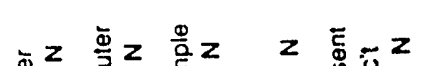

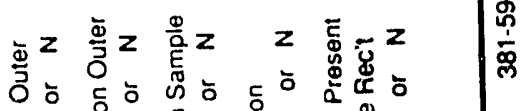

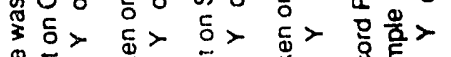

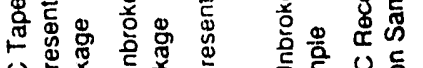

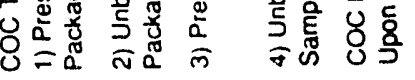

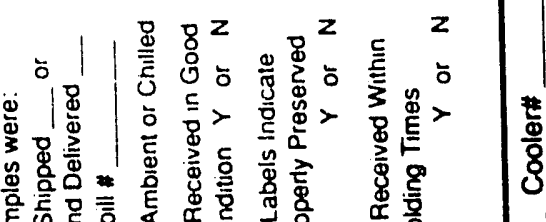

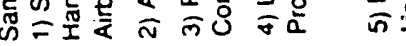

(2)

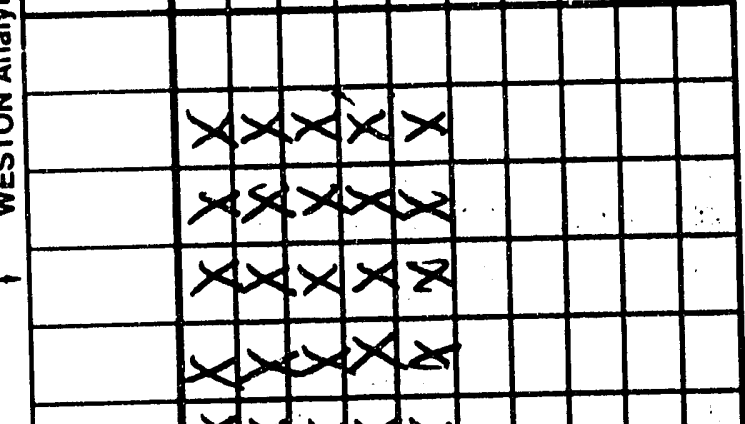

$x \times 4 \times 4 \times$

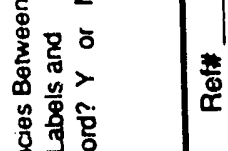

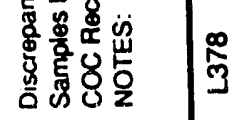

$\stackrel{\stackrel{\oplus}{E}}{\mathrm{E}}$

$x=4 \times 4 \times 2$

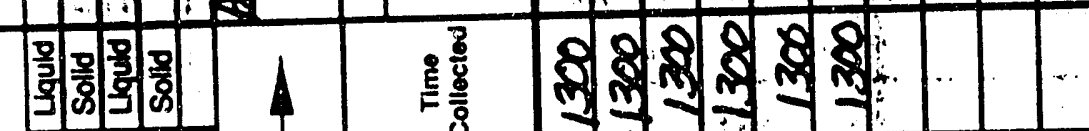

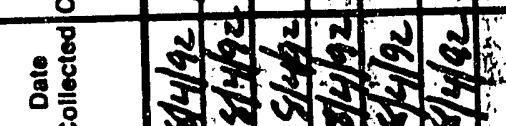

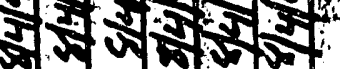

: vmving

\begin{tabular}{c|c|c|}
\hline \\
\hline
\end{tabular}

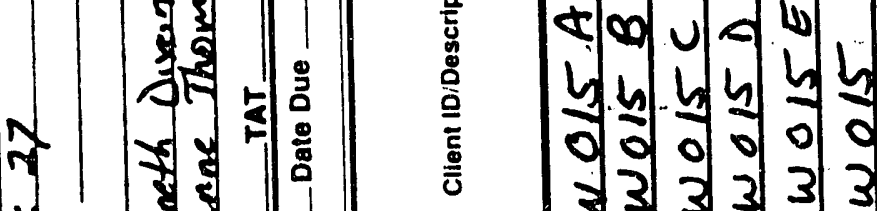

3 3. 3 ? 33

जैजले ज्ञ बै

畩

*

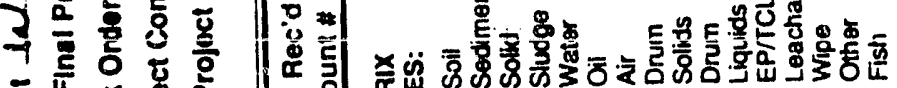

E

8

$\frac{n}{3}$

$\frac{8}{8}$ ప

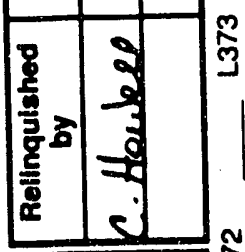

है

ज्ञ

| 


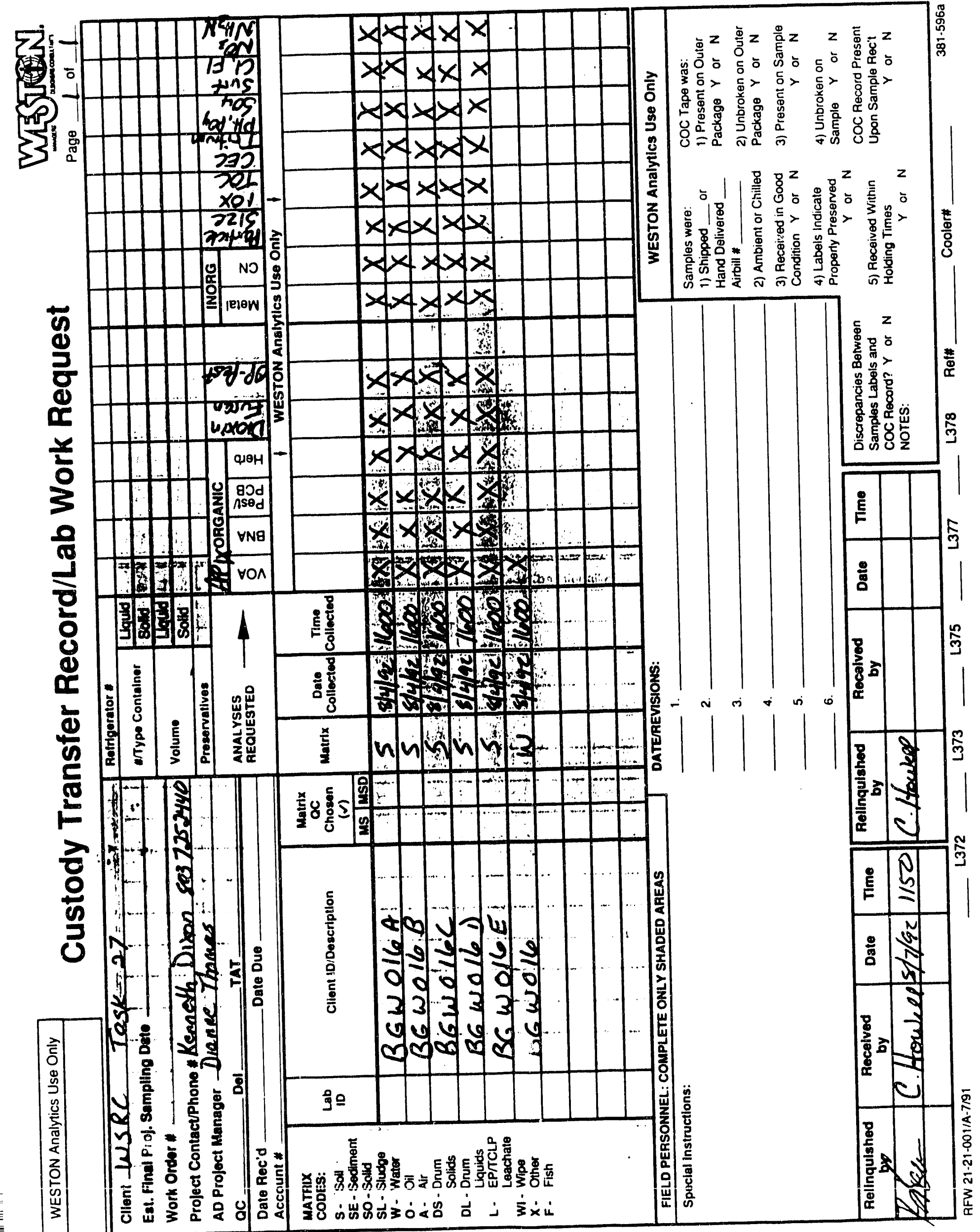




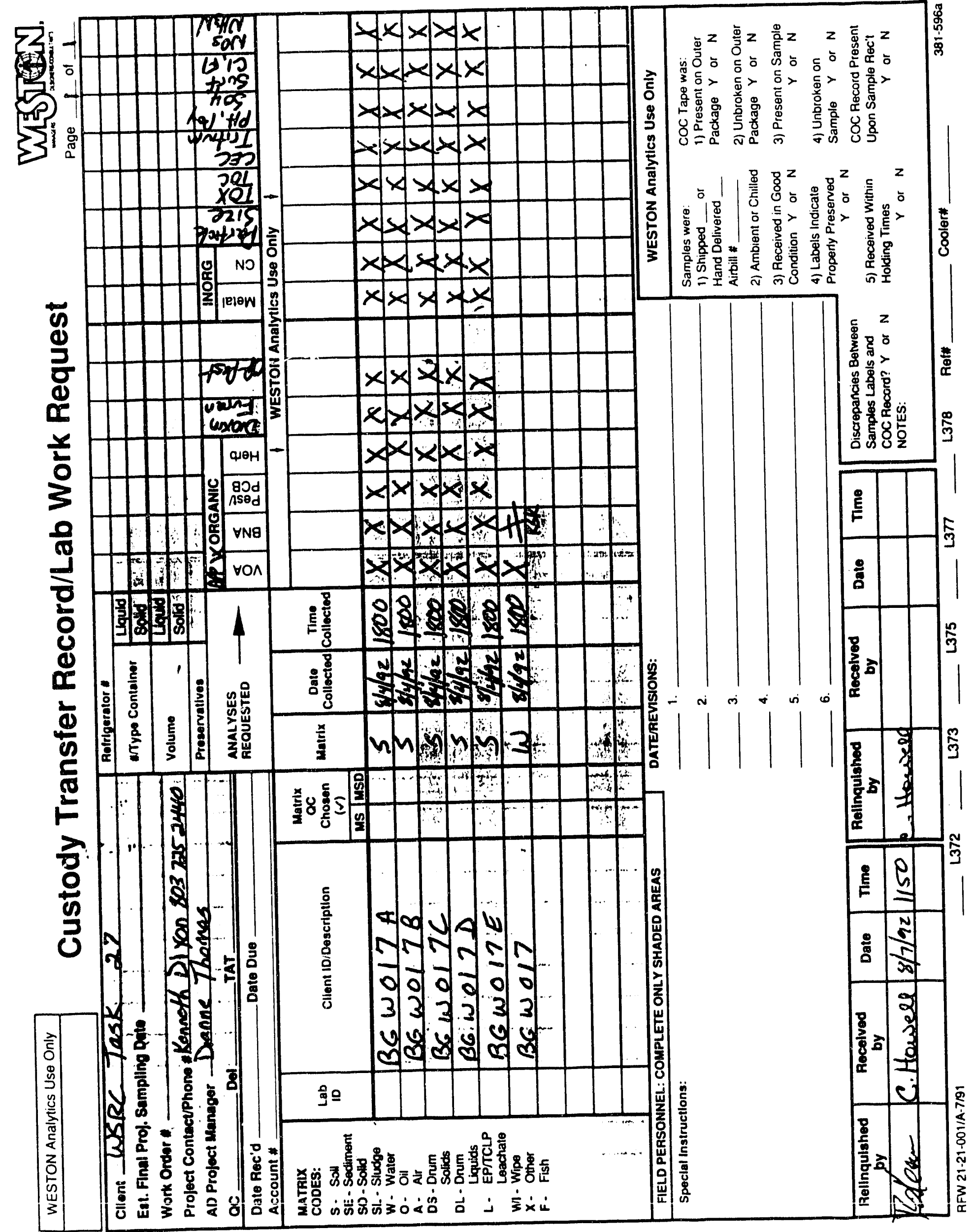




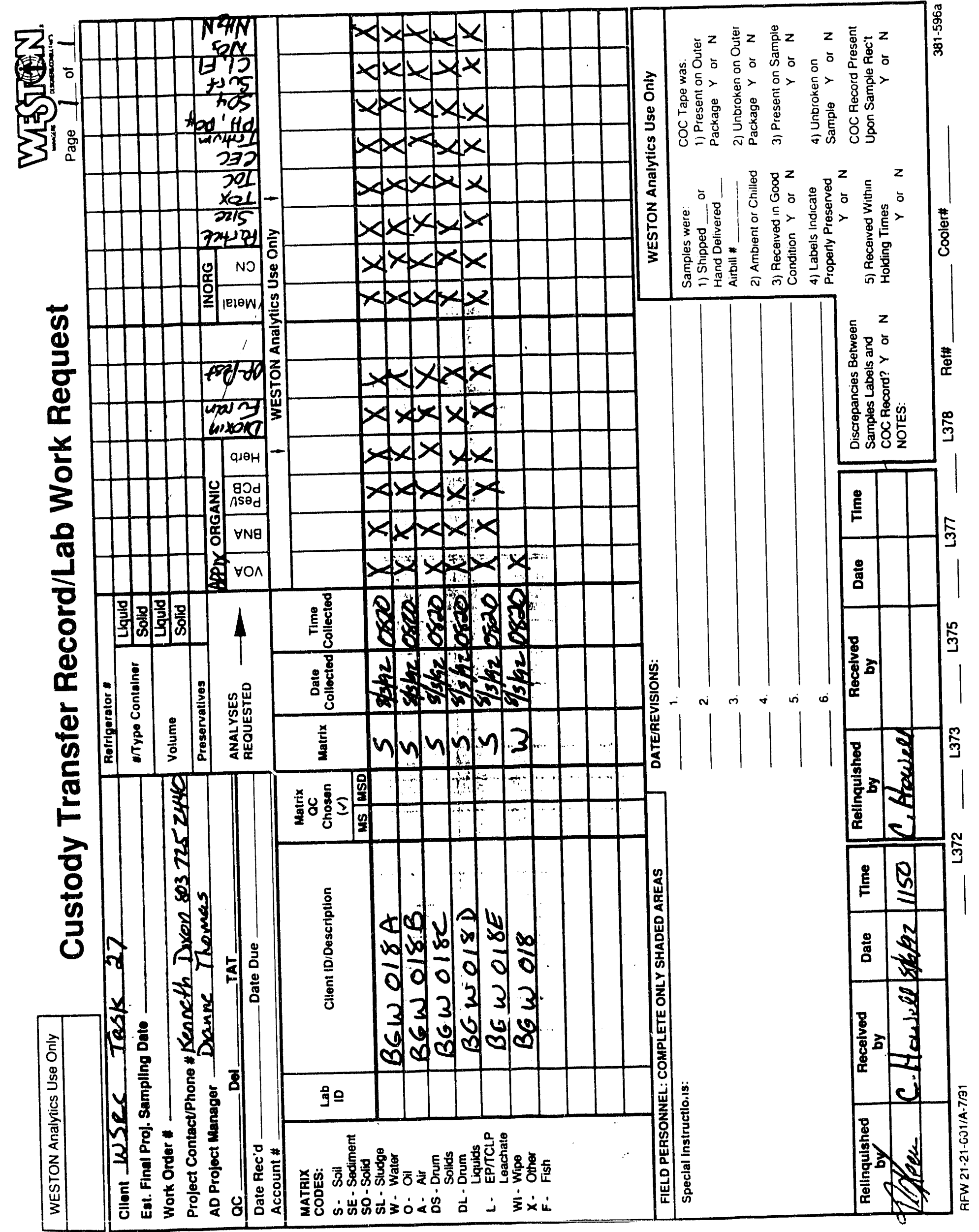




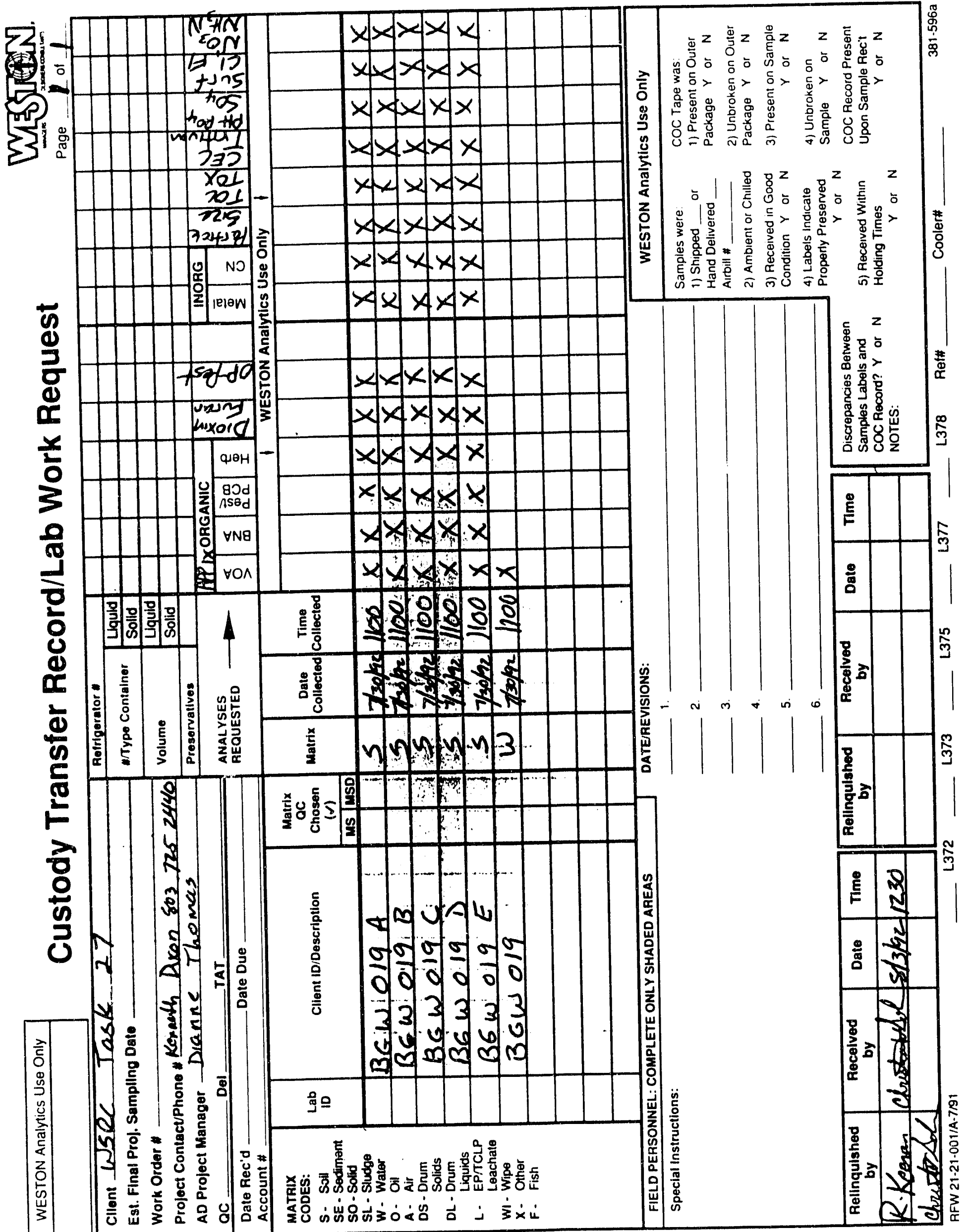




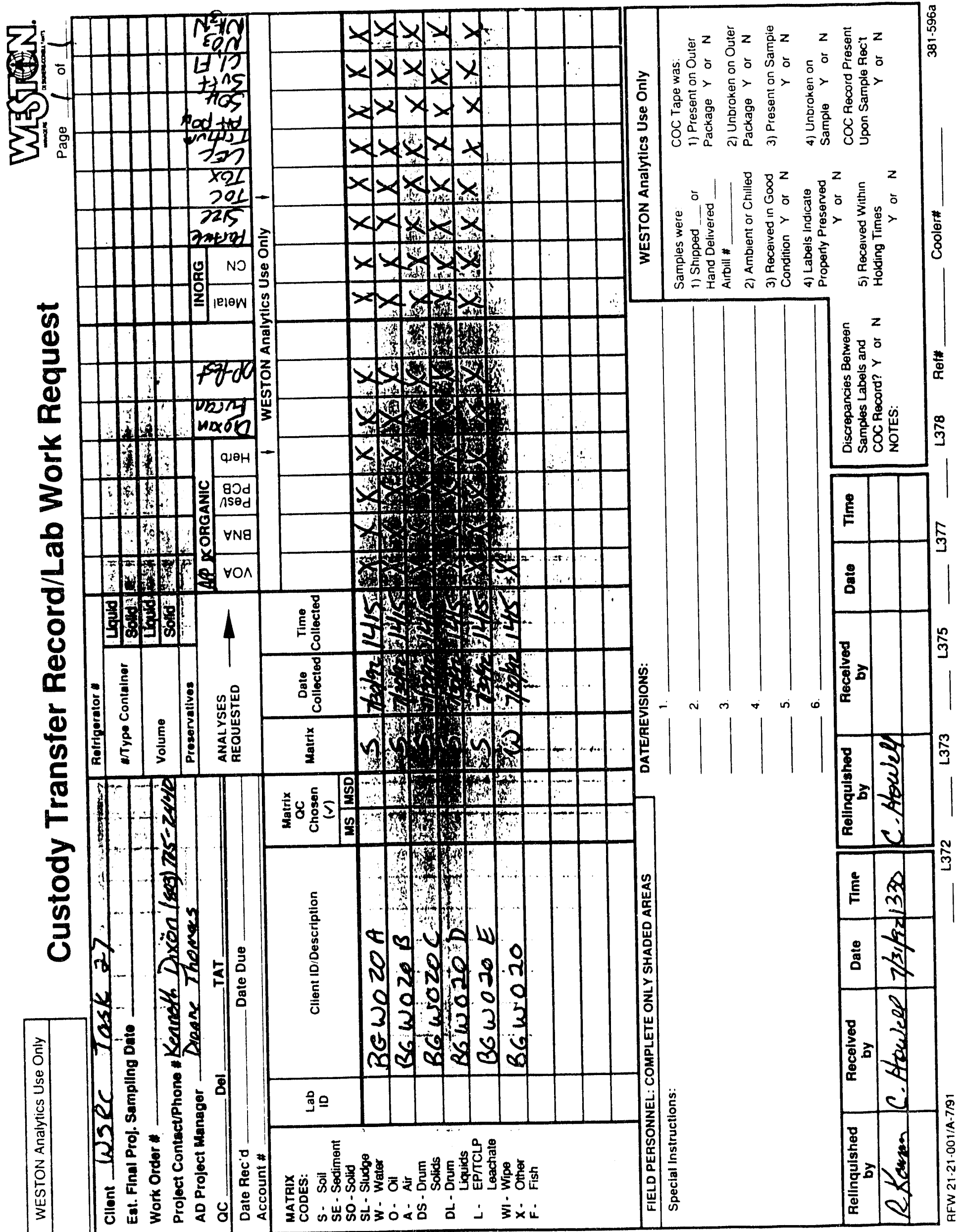




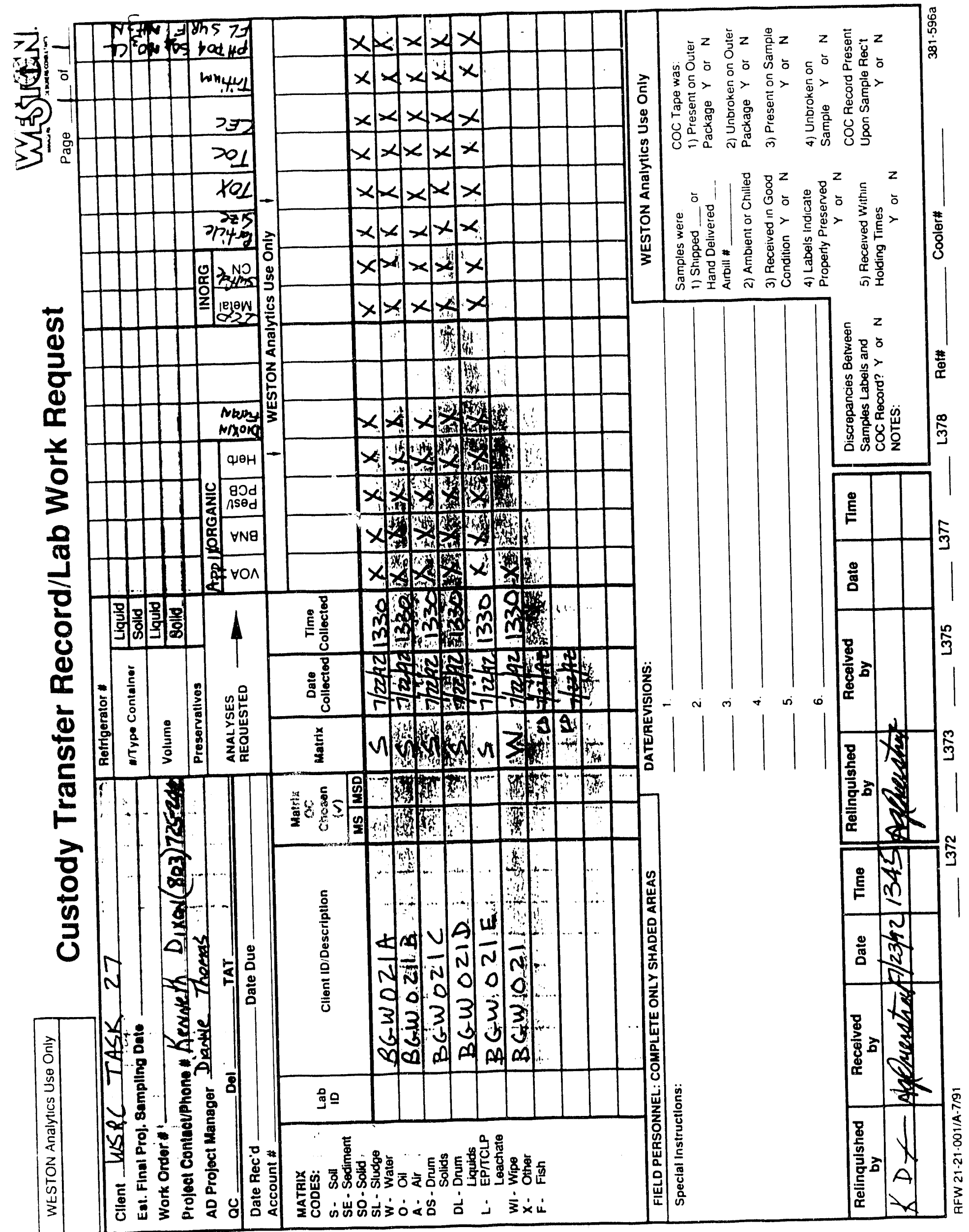




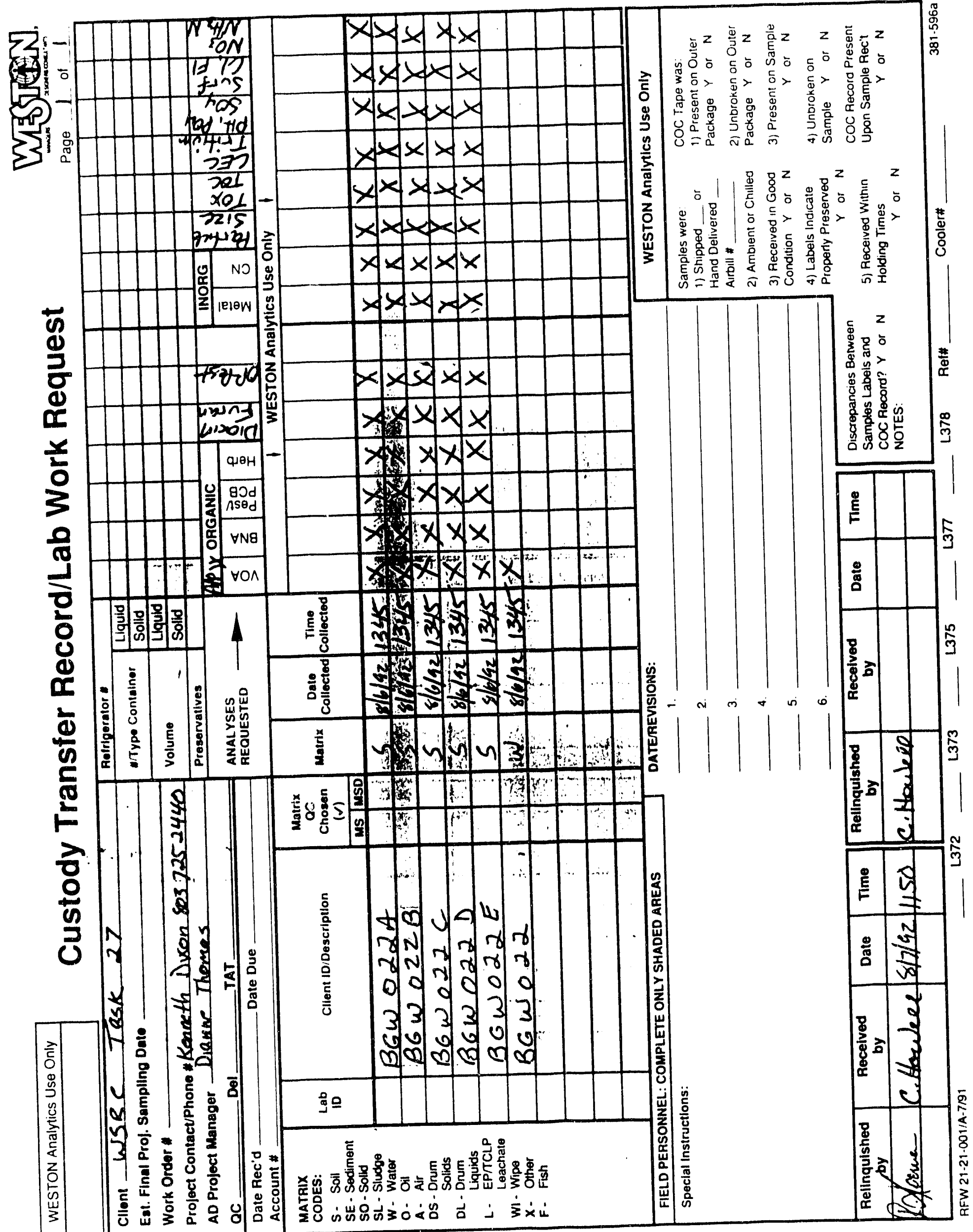




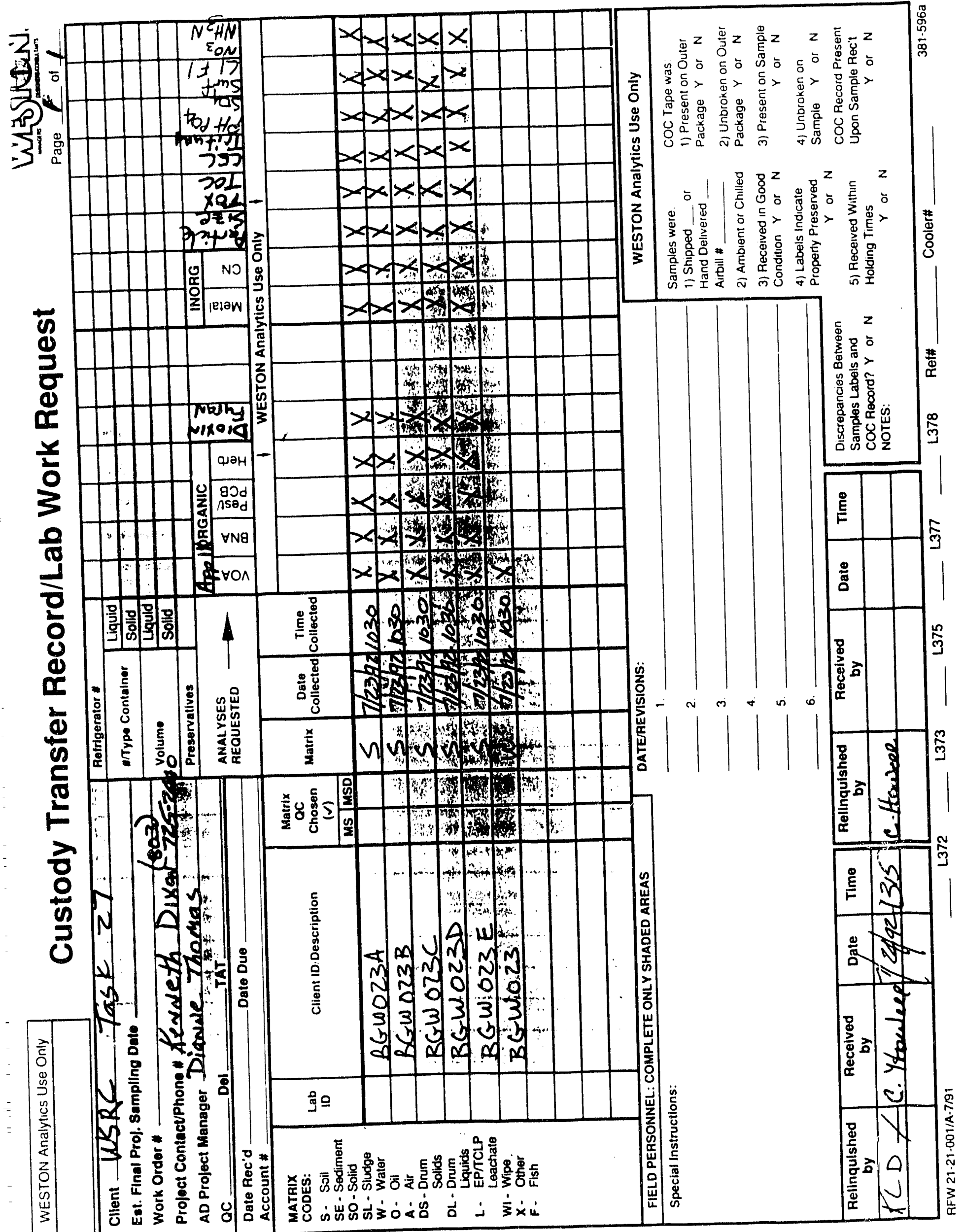




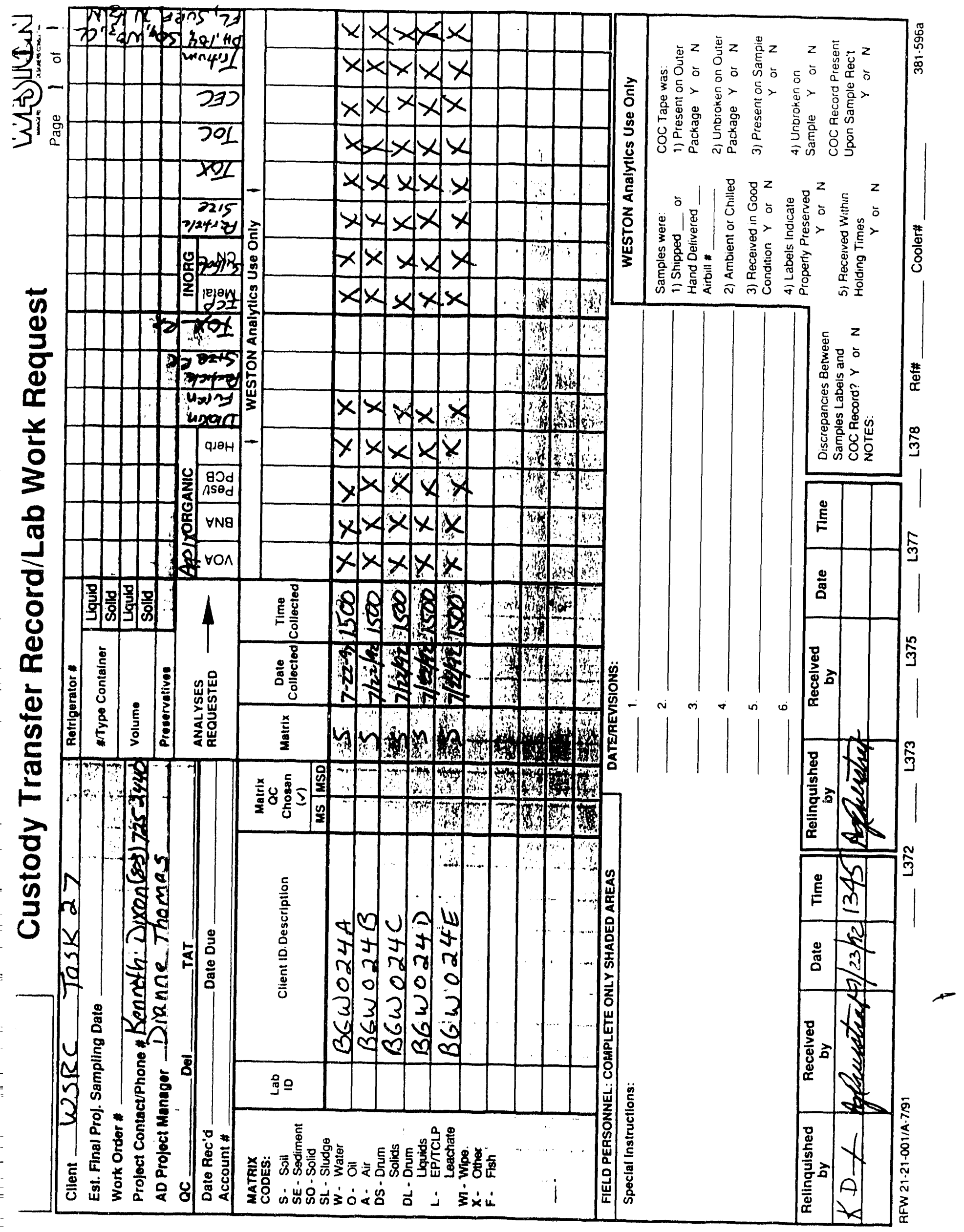




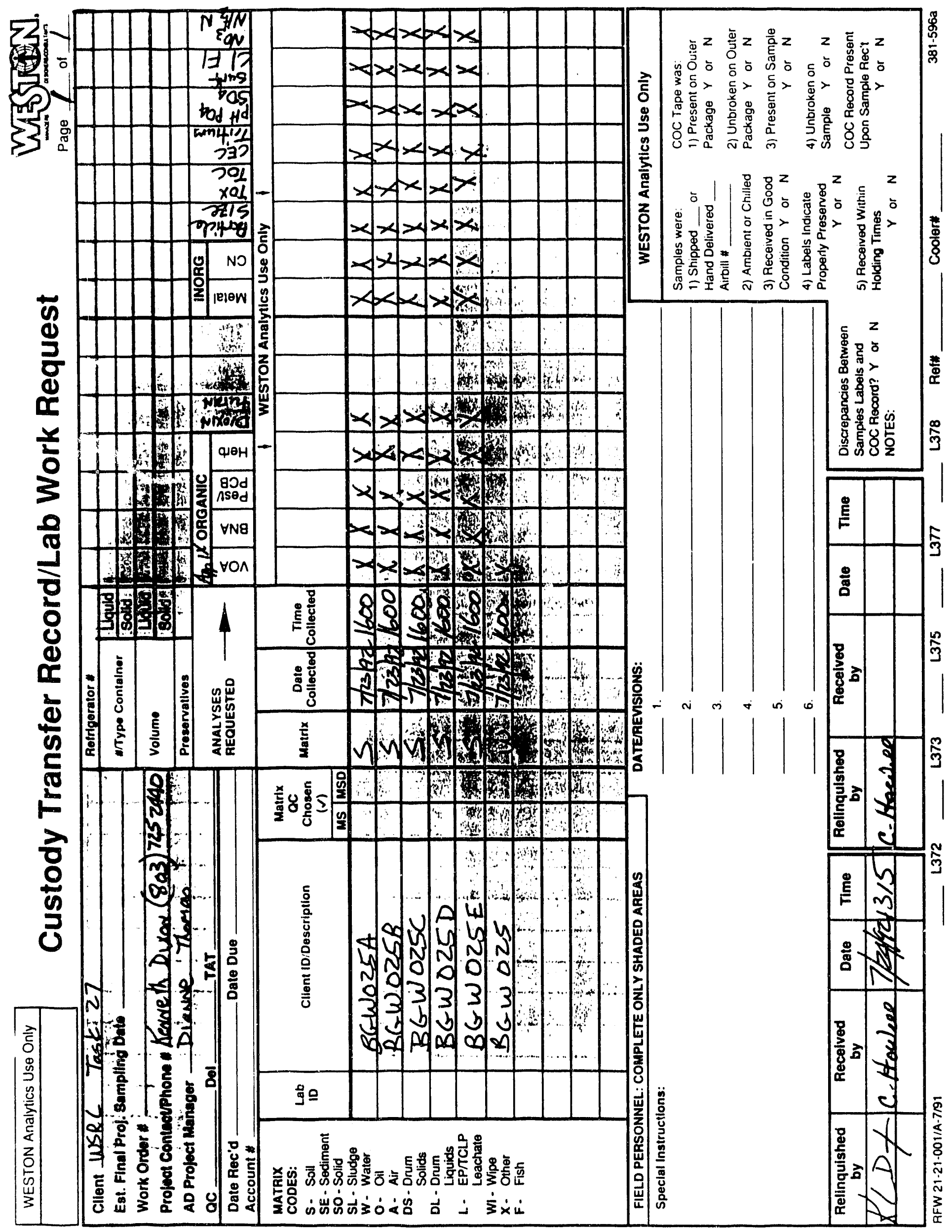




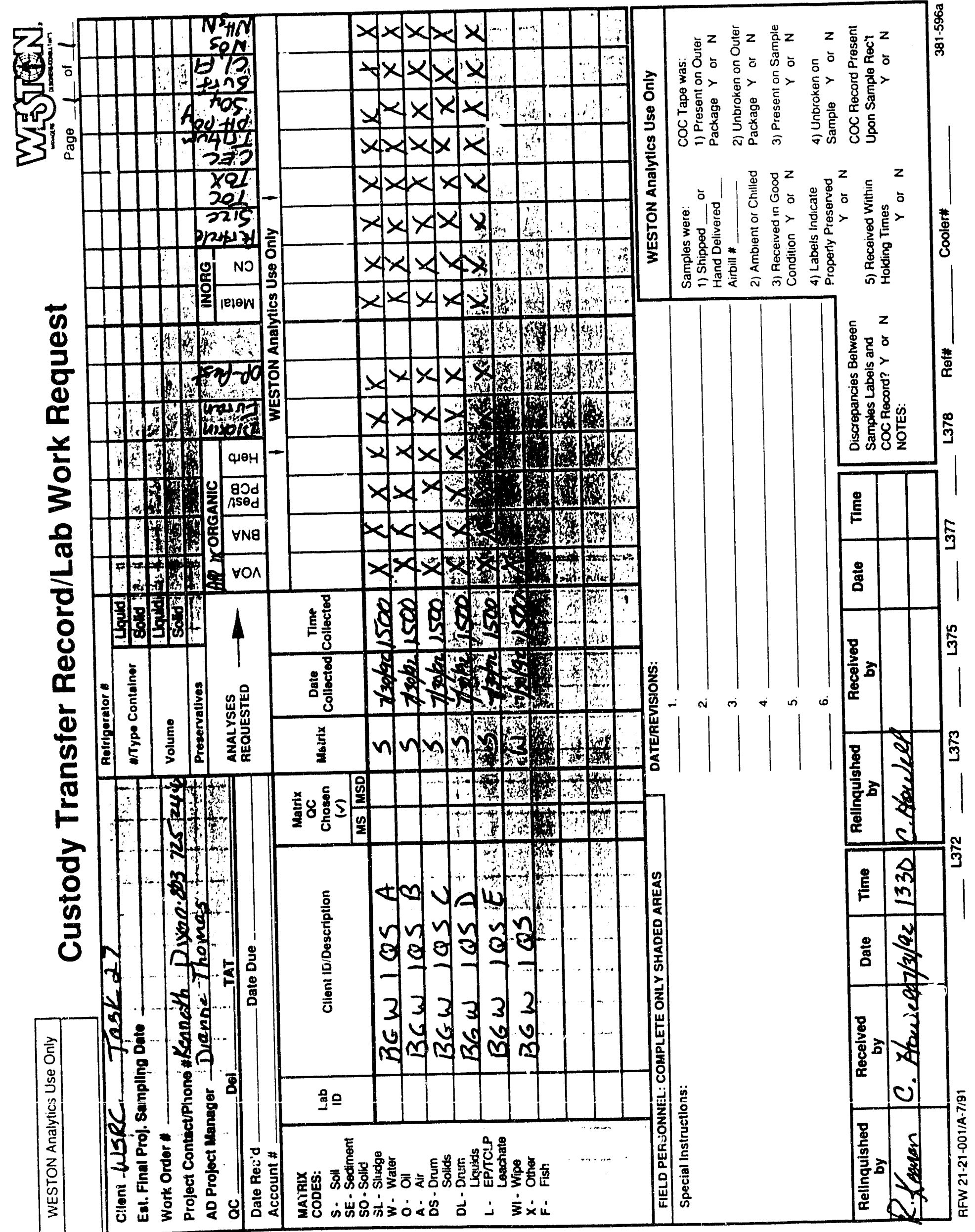


待
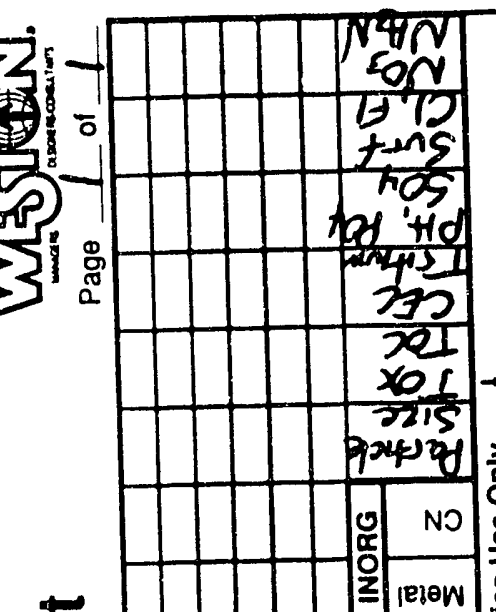

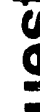

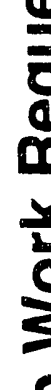

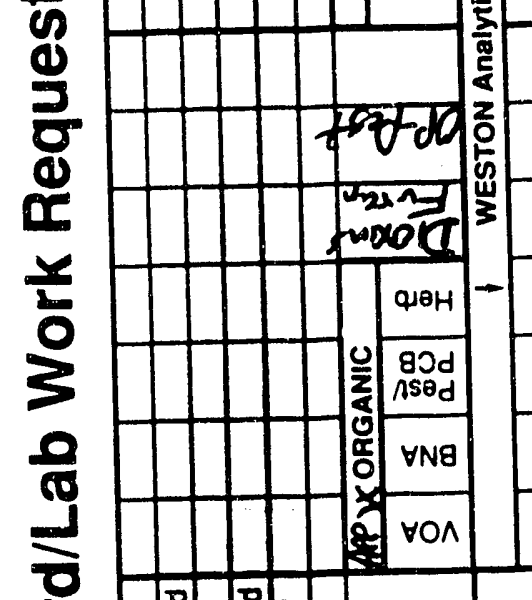

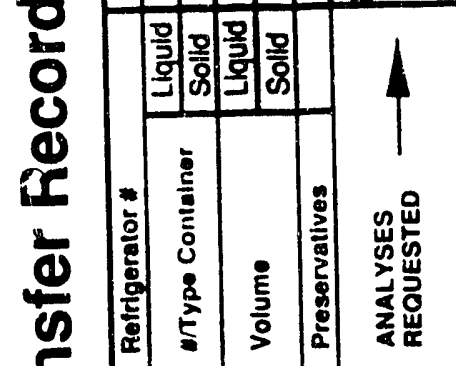

离

||

प

M

ฟ

З

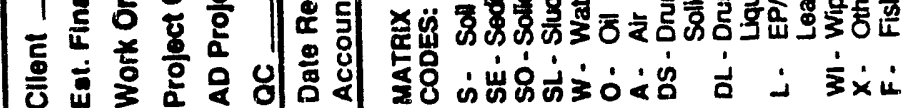

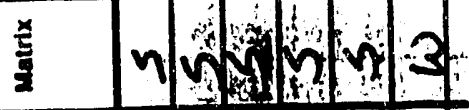

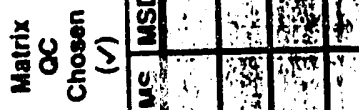

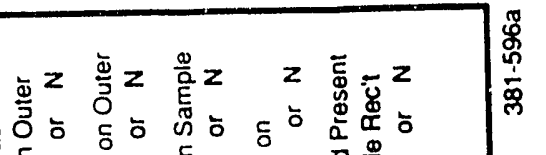

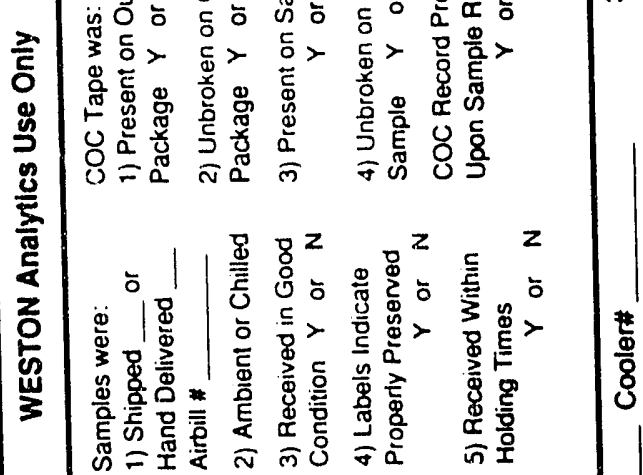

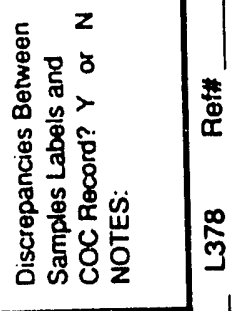

Е

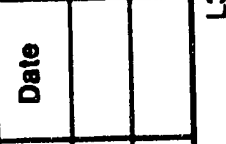

8

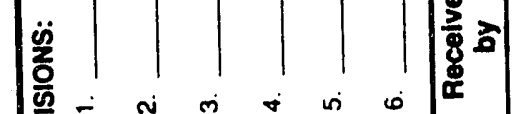

.

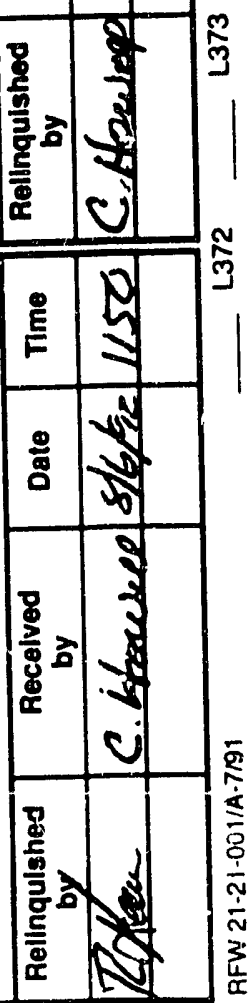




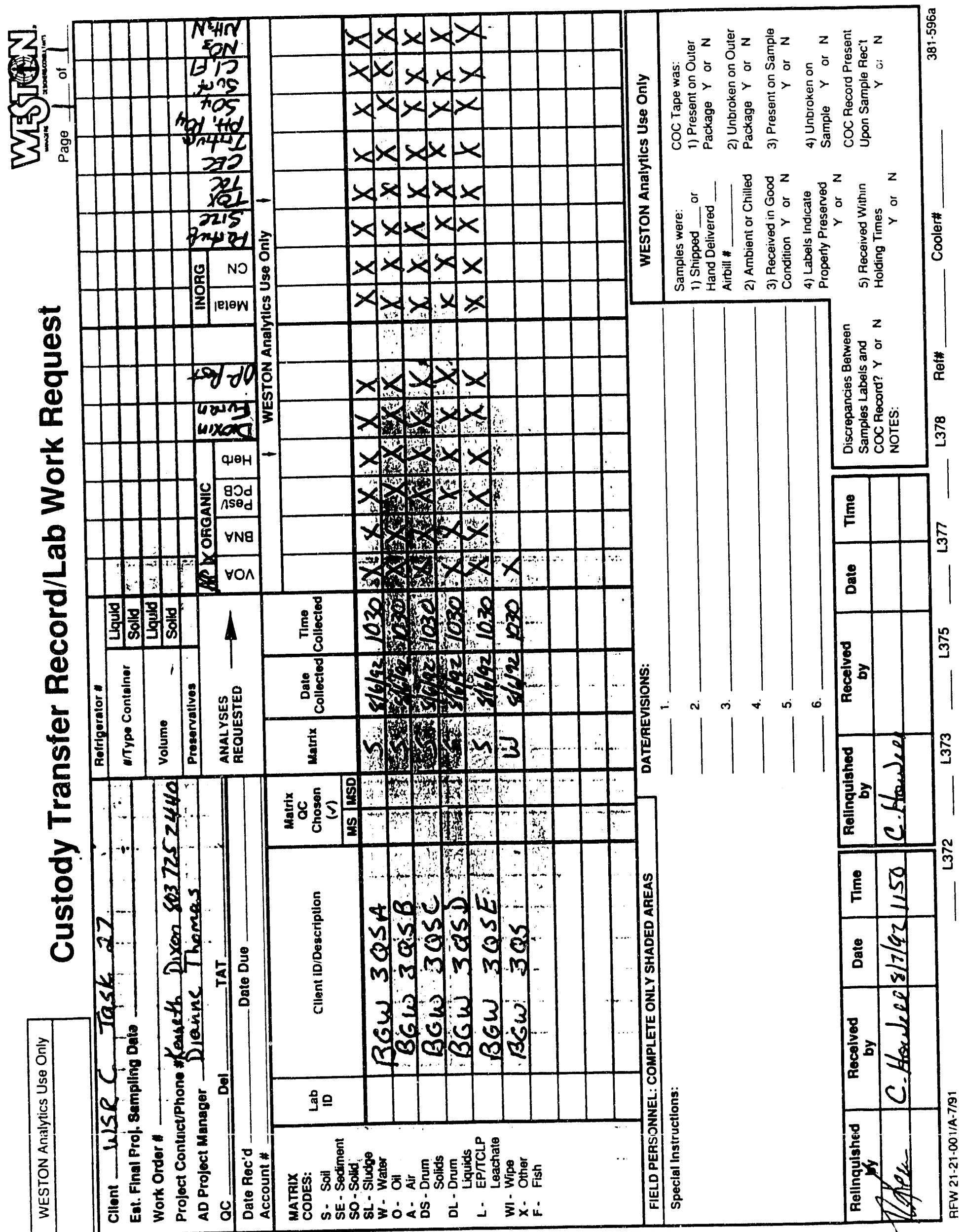




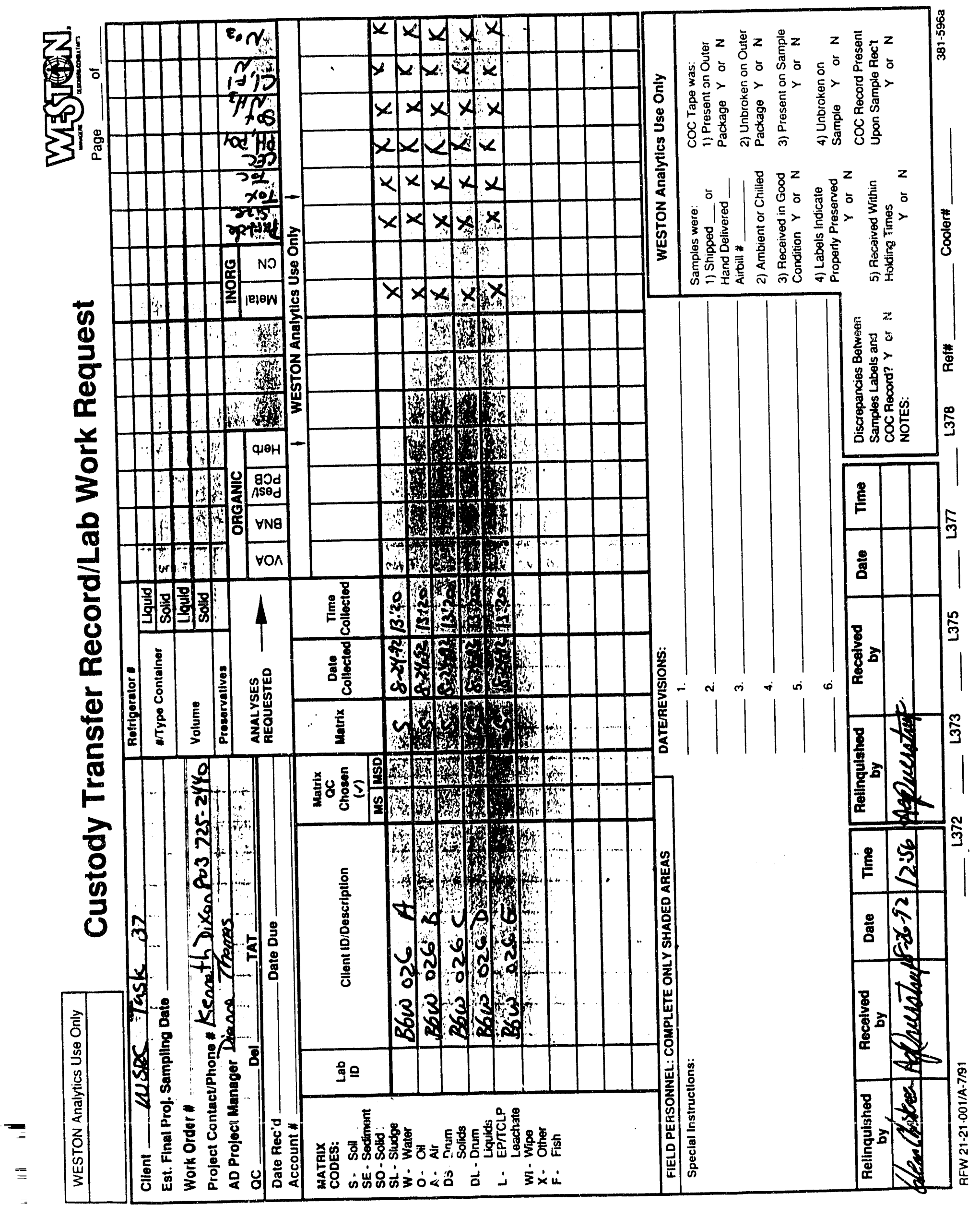




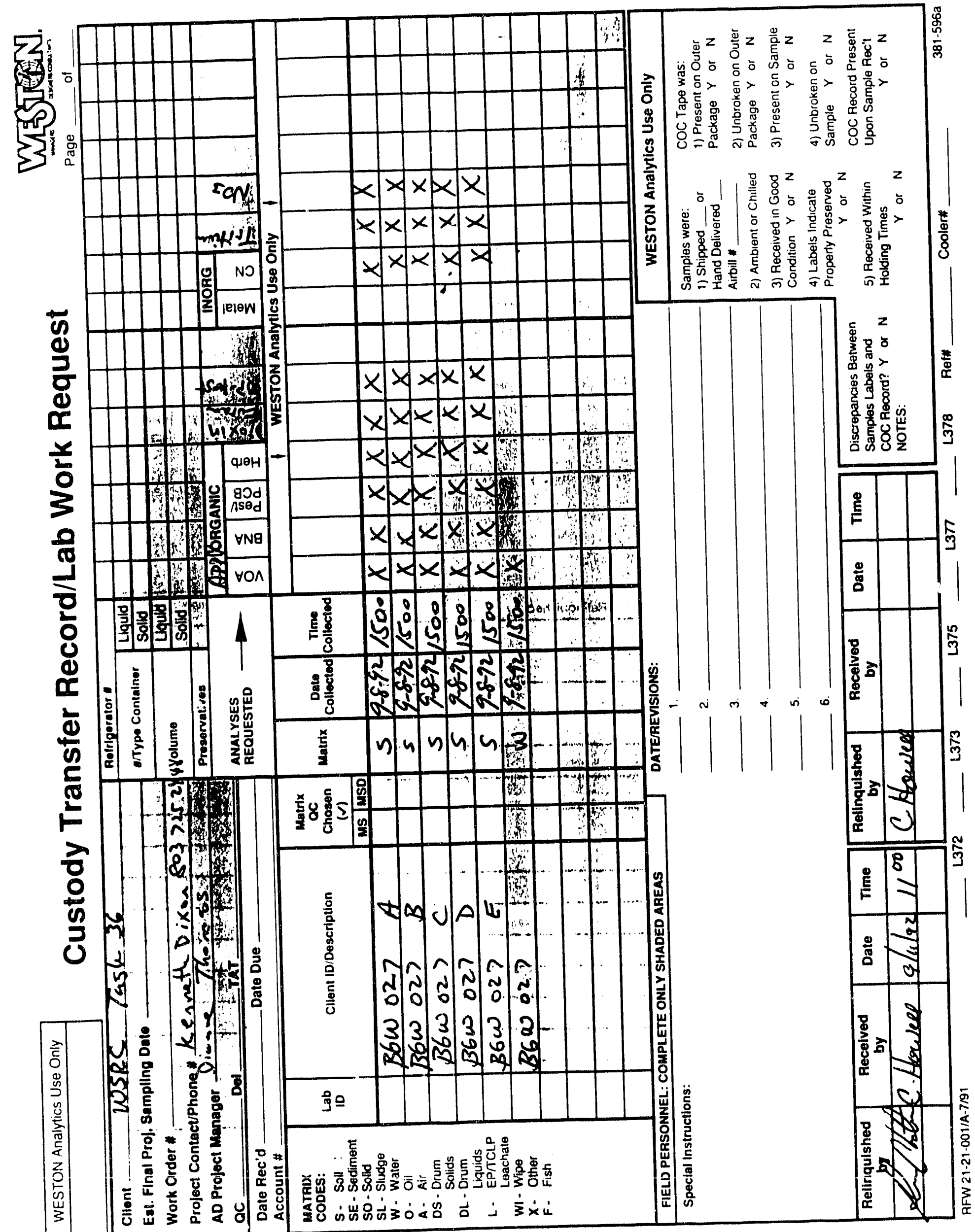




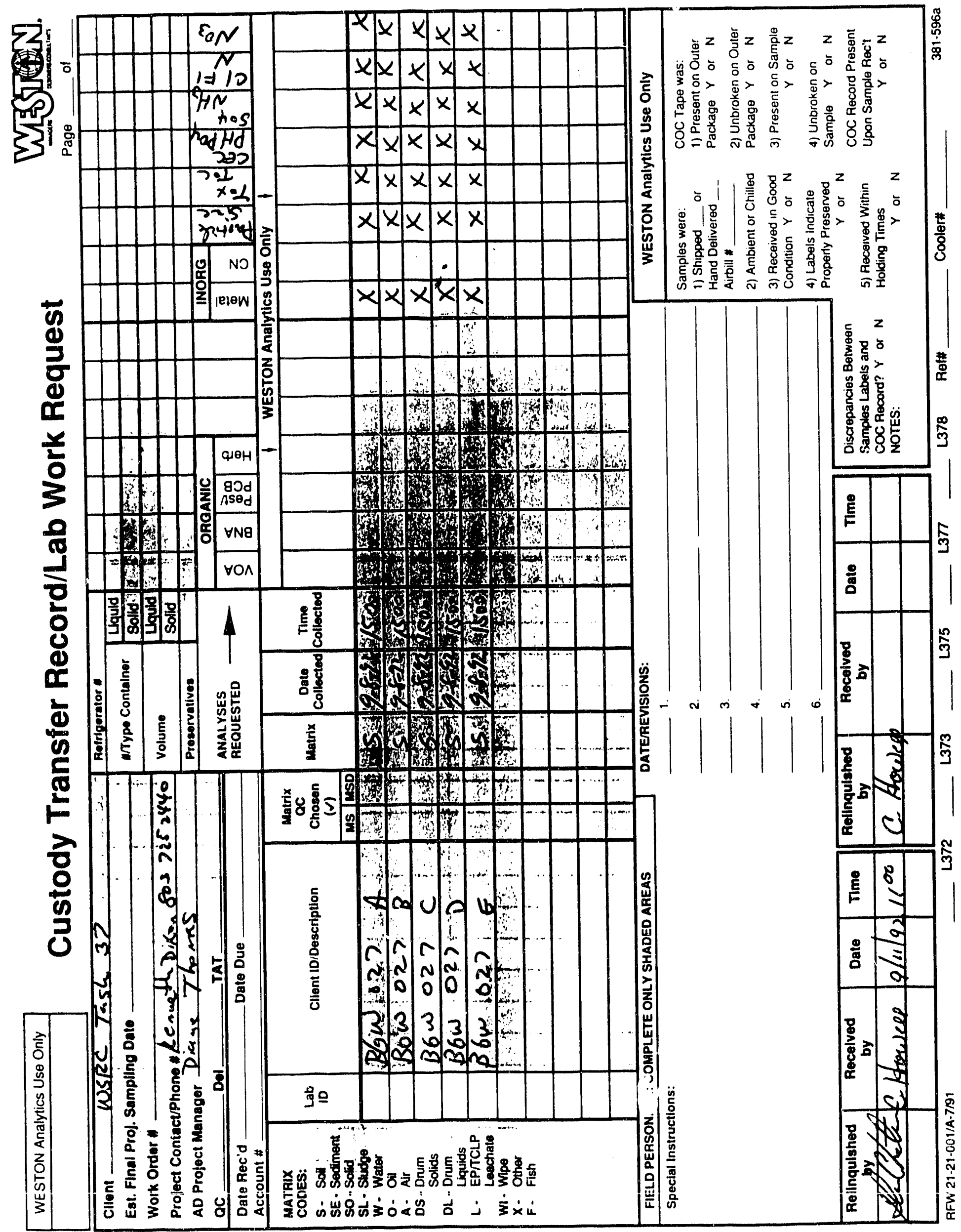




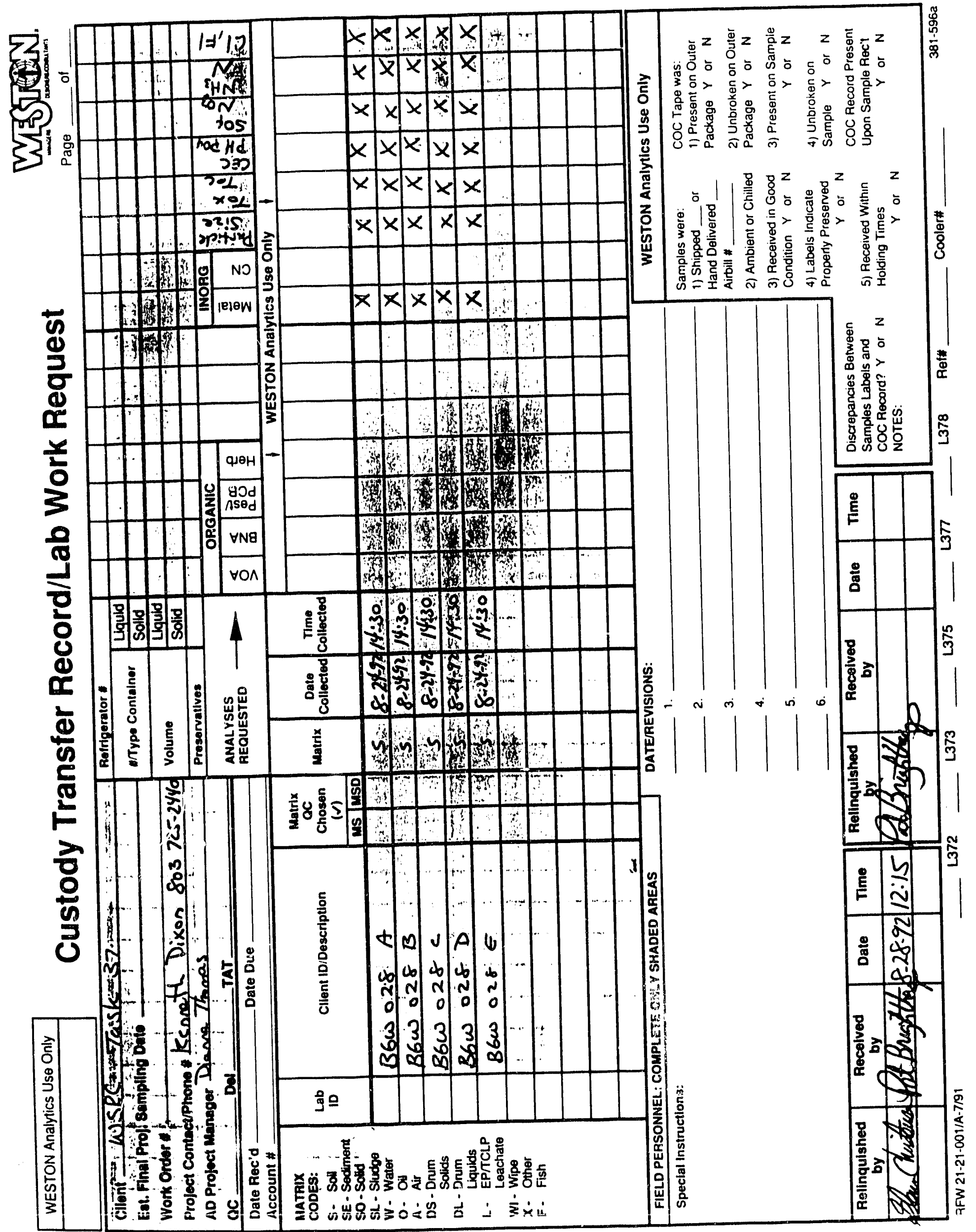


遥

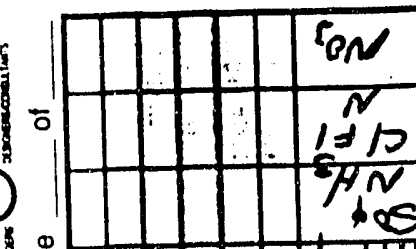

这

thod

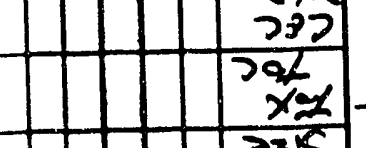

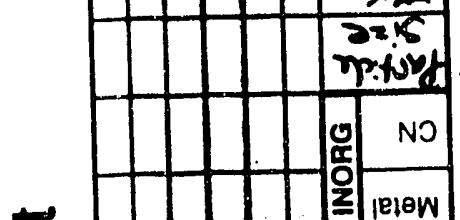

5

$\frac{9}{8}$

要

. L

MO

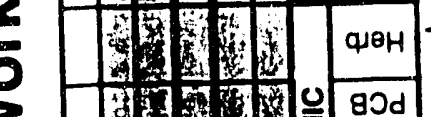

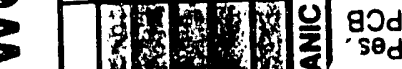

은

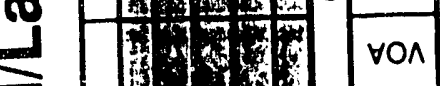

은

范

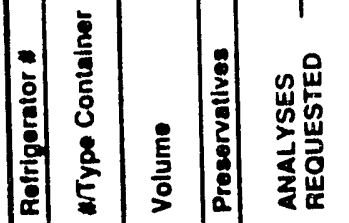

要

๘

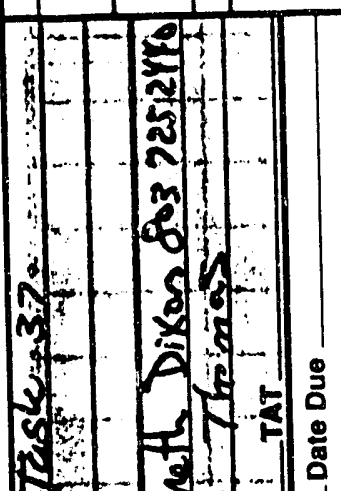

$4-8$.

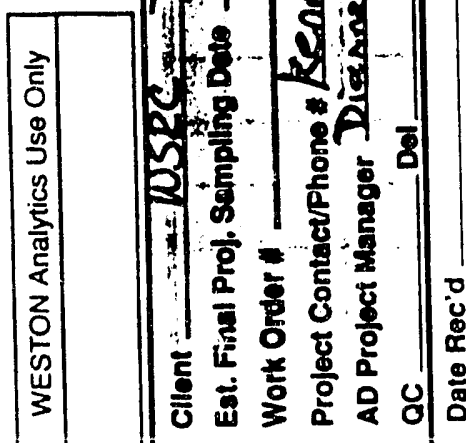

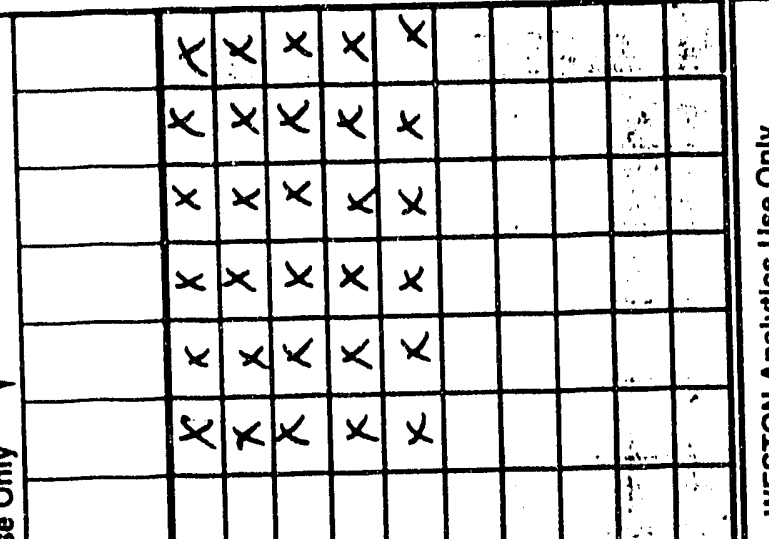

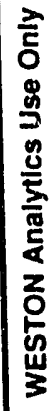

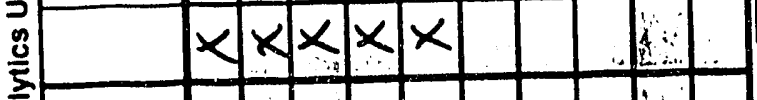

$x \times x \times x$

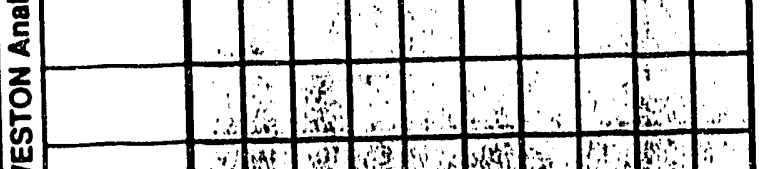

30200

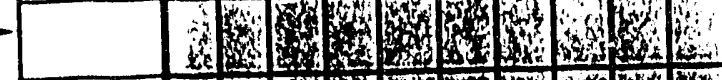
(x)

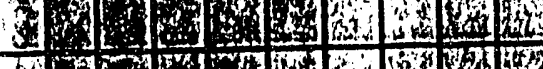
3. 12,420

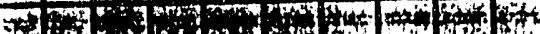

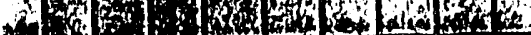

竞要

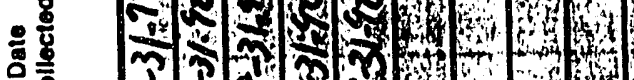

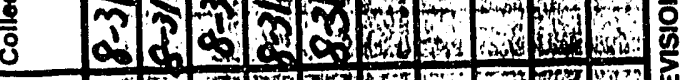

wow 3. T.

每 施

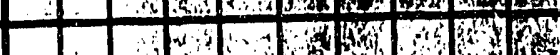
崖

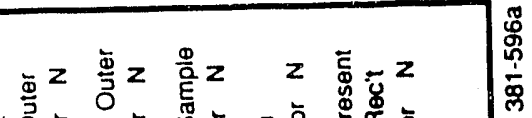$$
\text { में }
$$

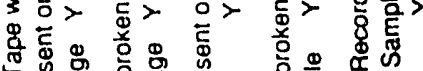

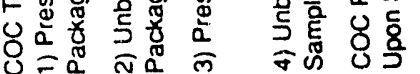

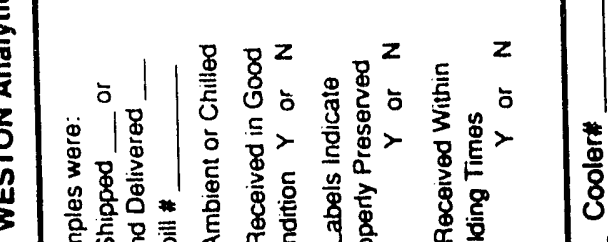

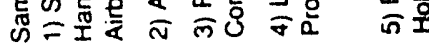
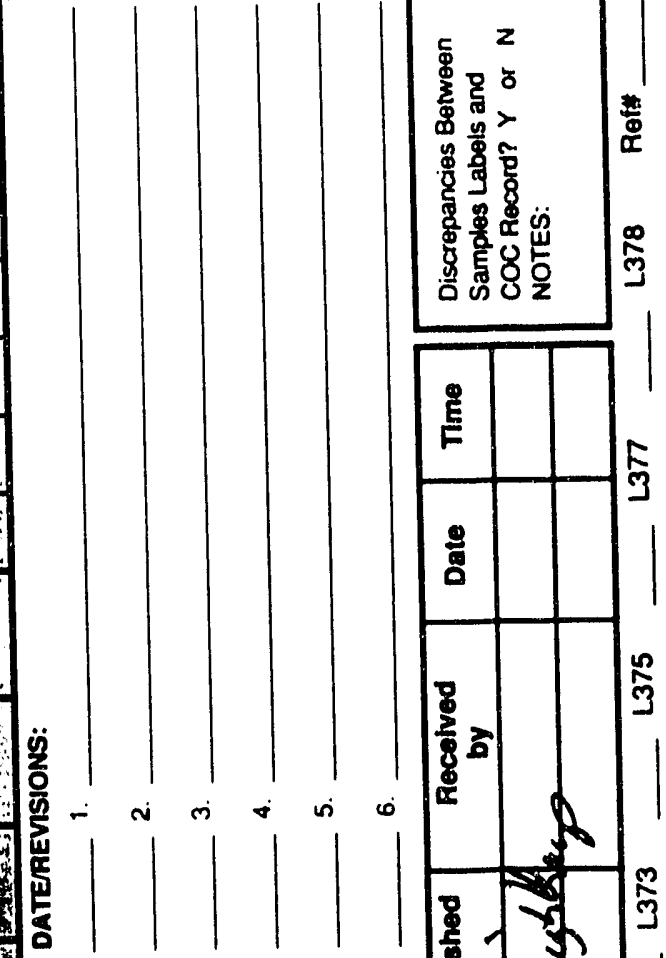
这

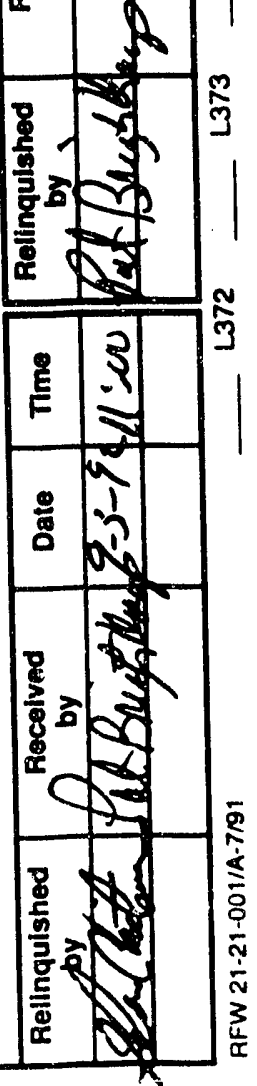




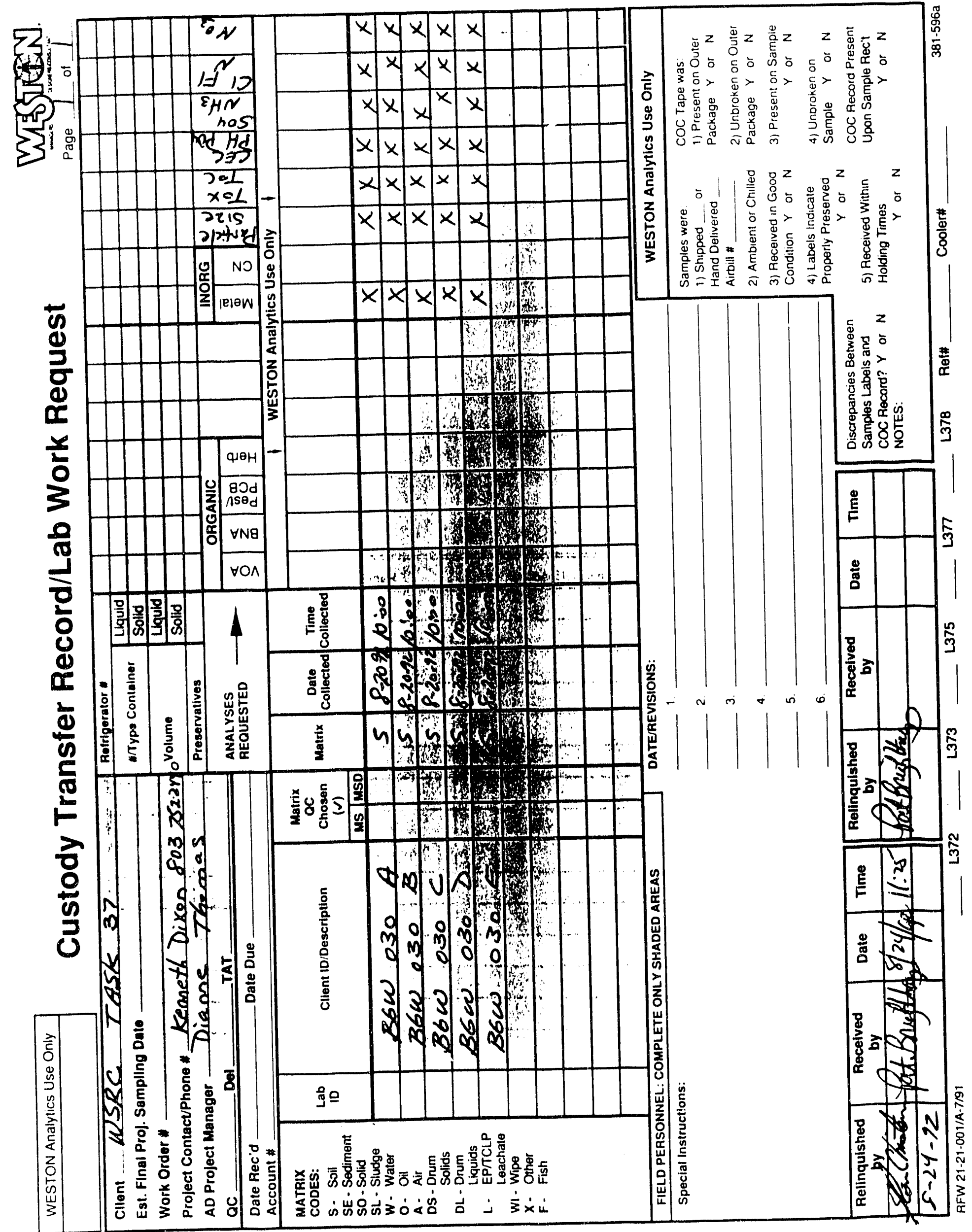




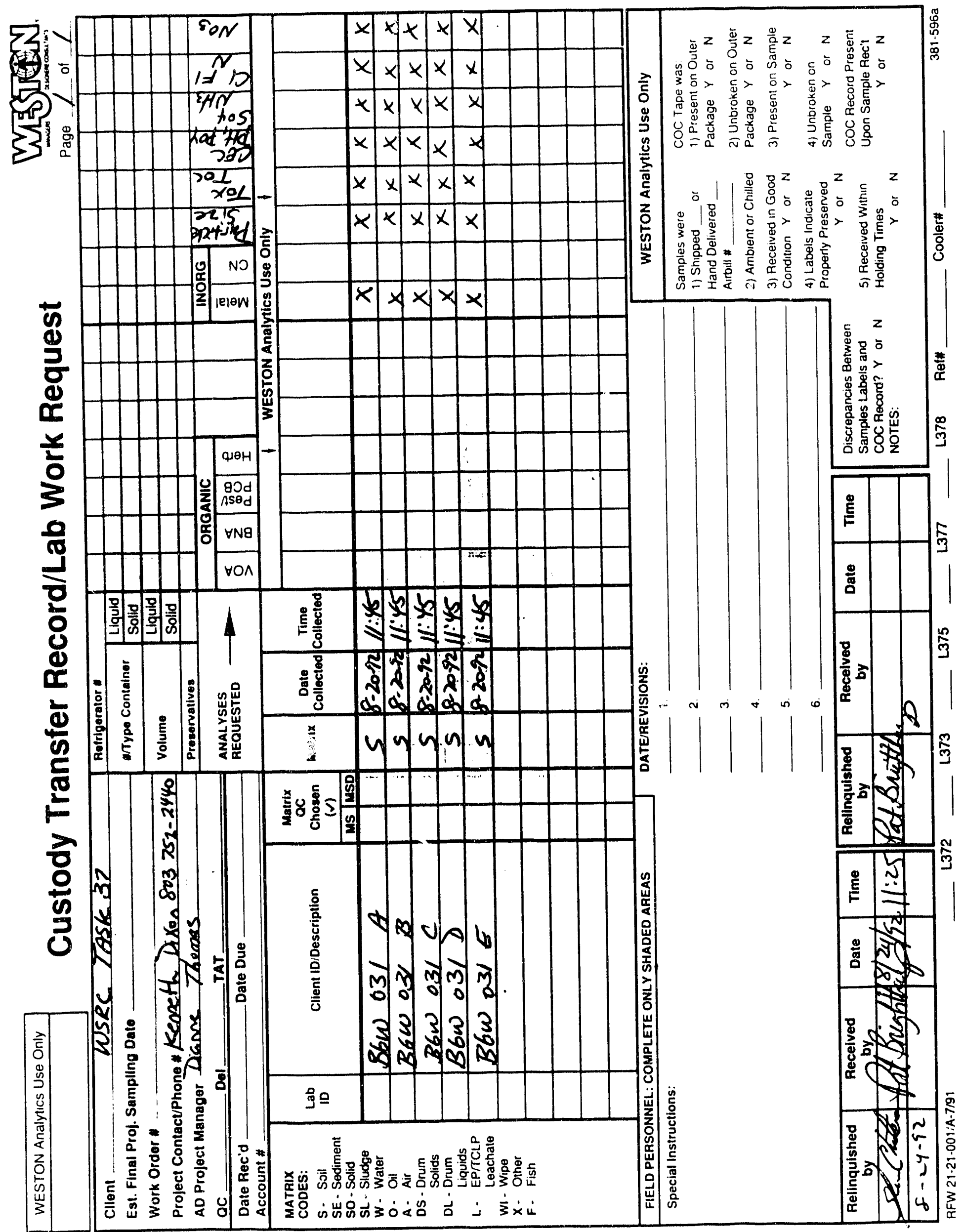




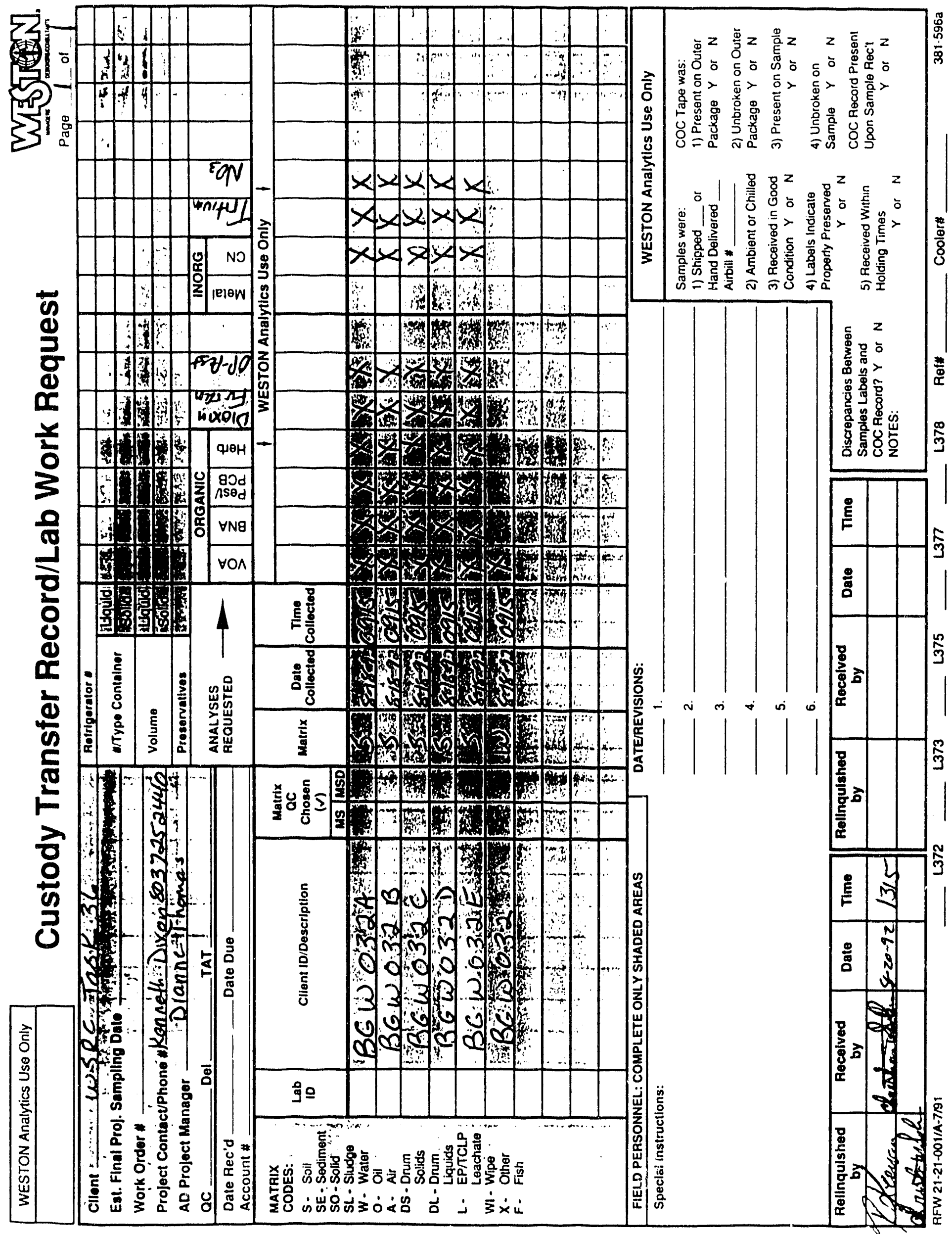




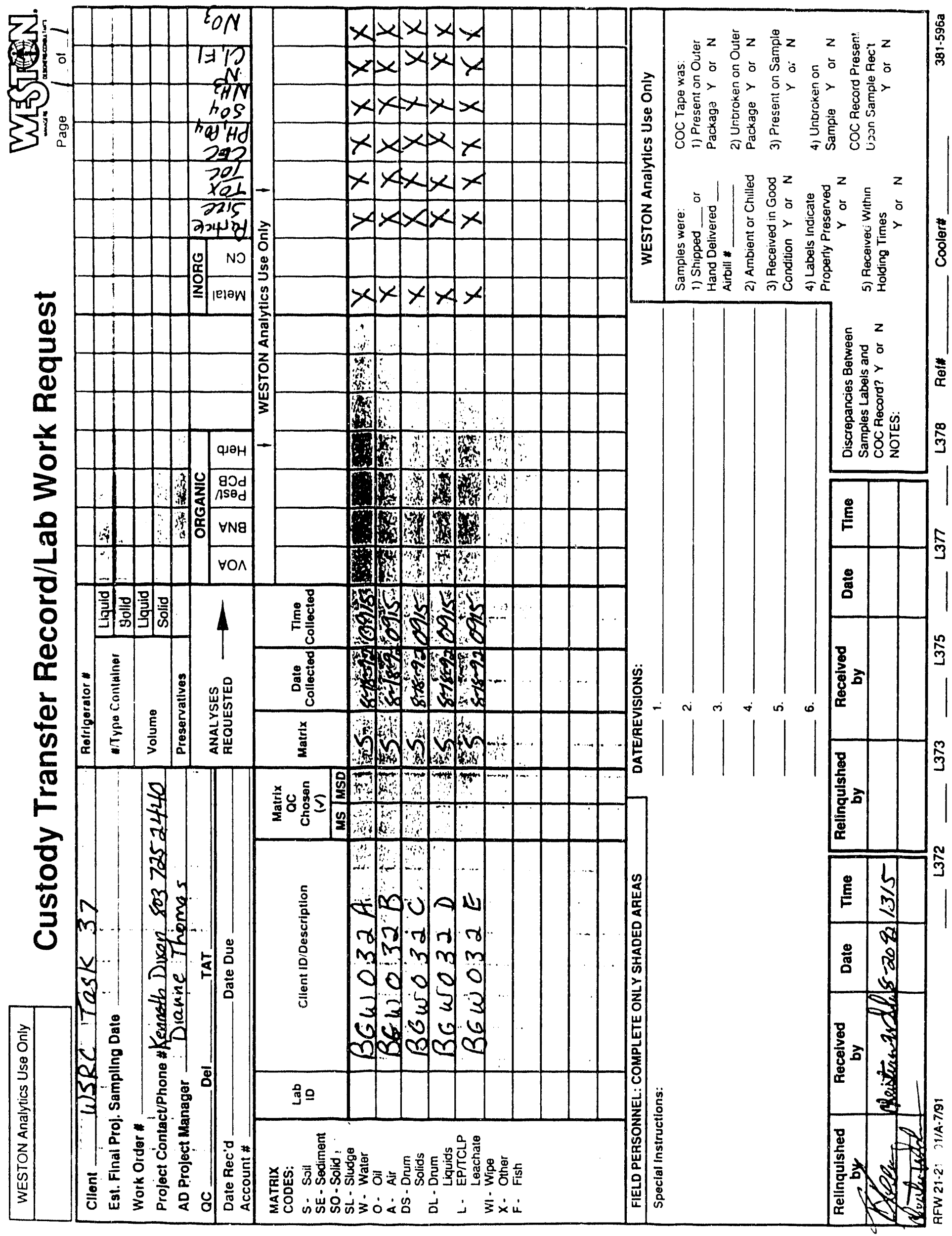




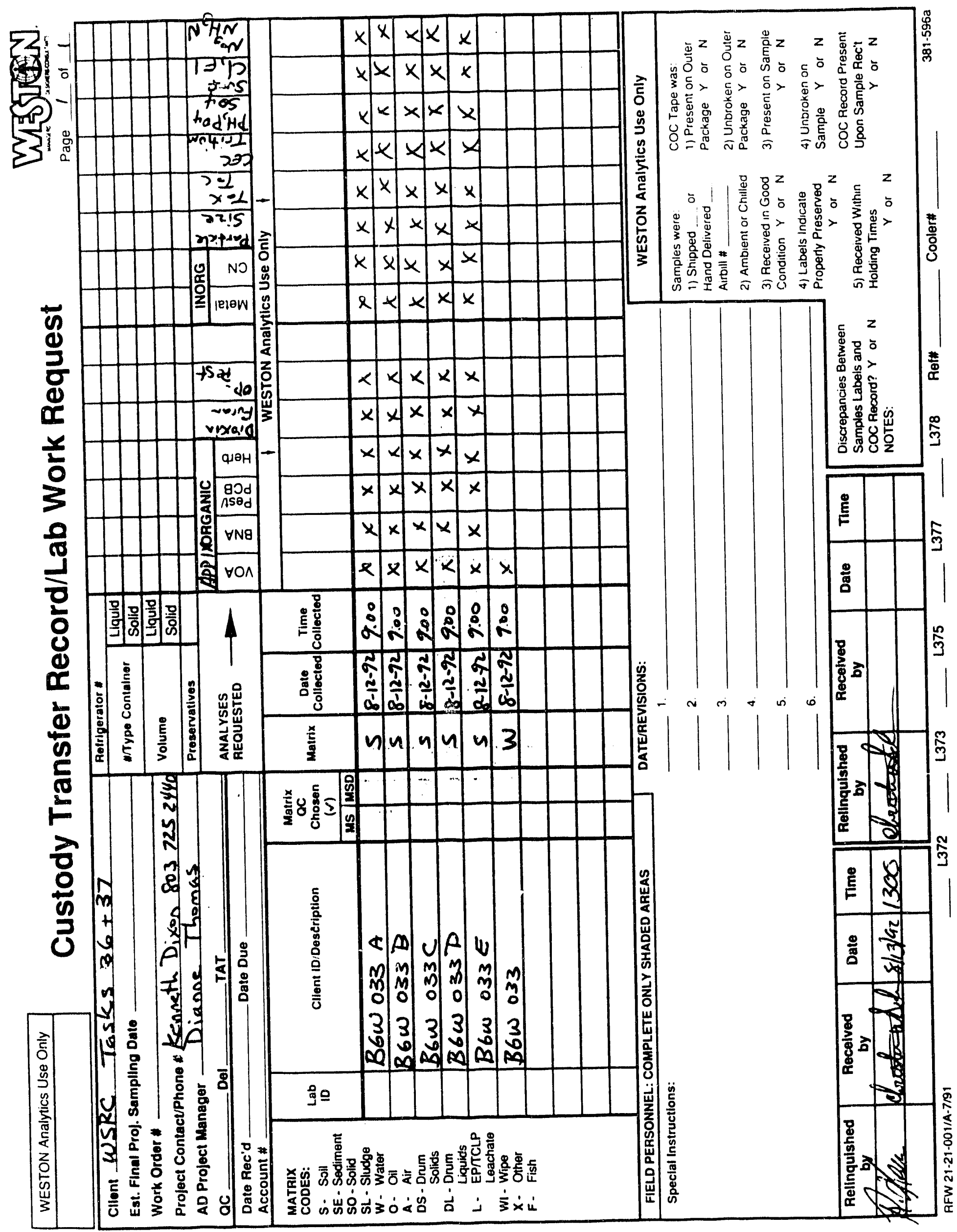




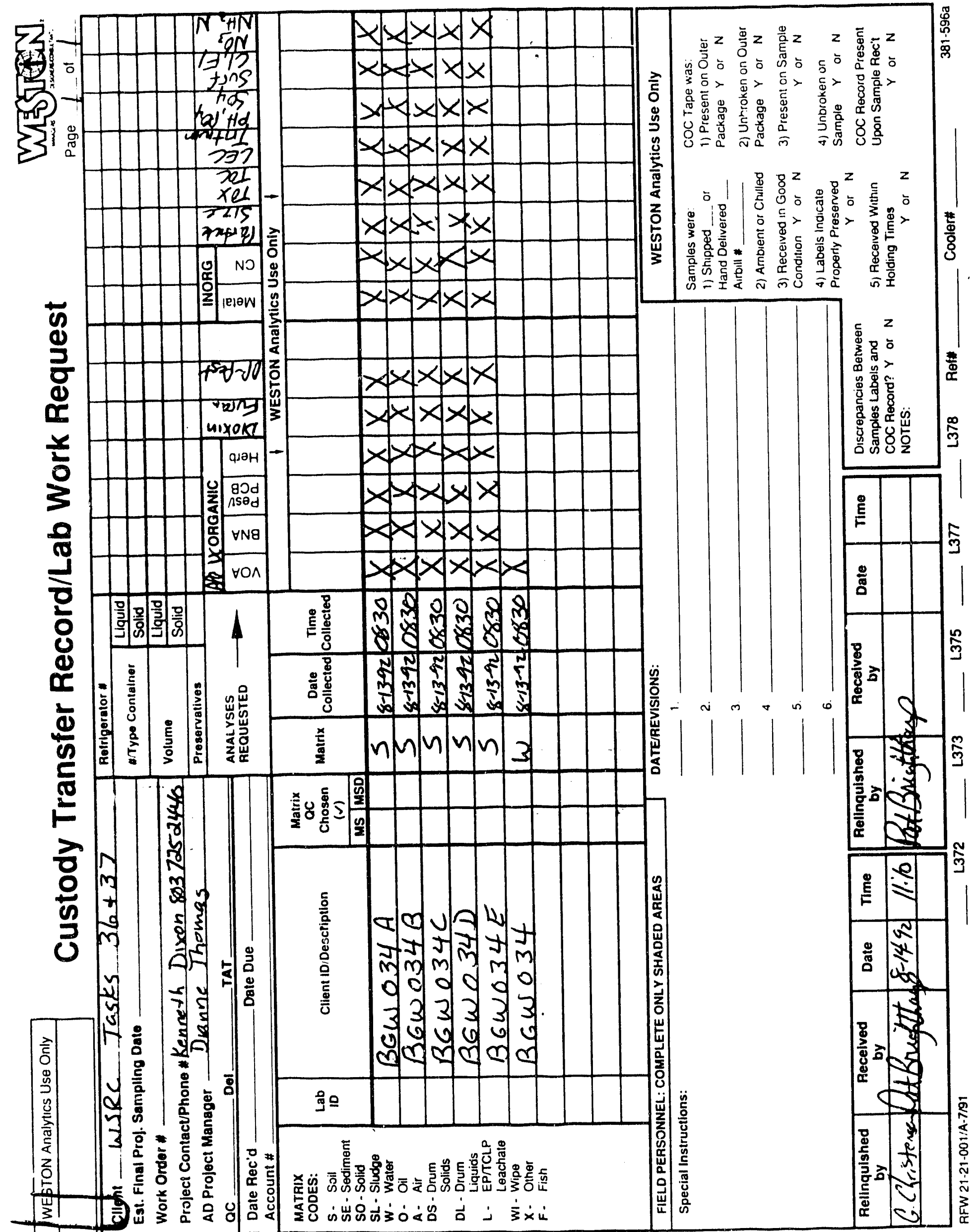




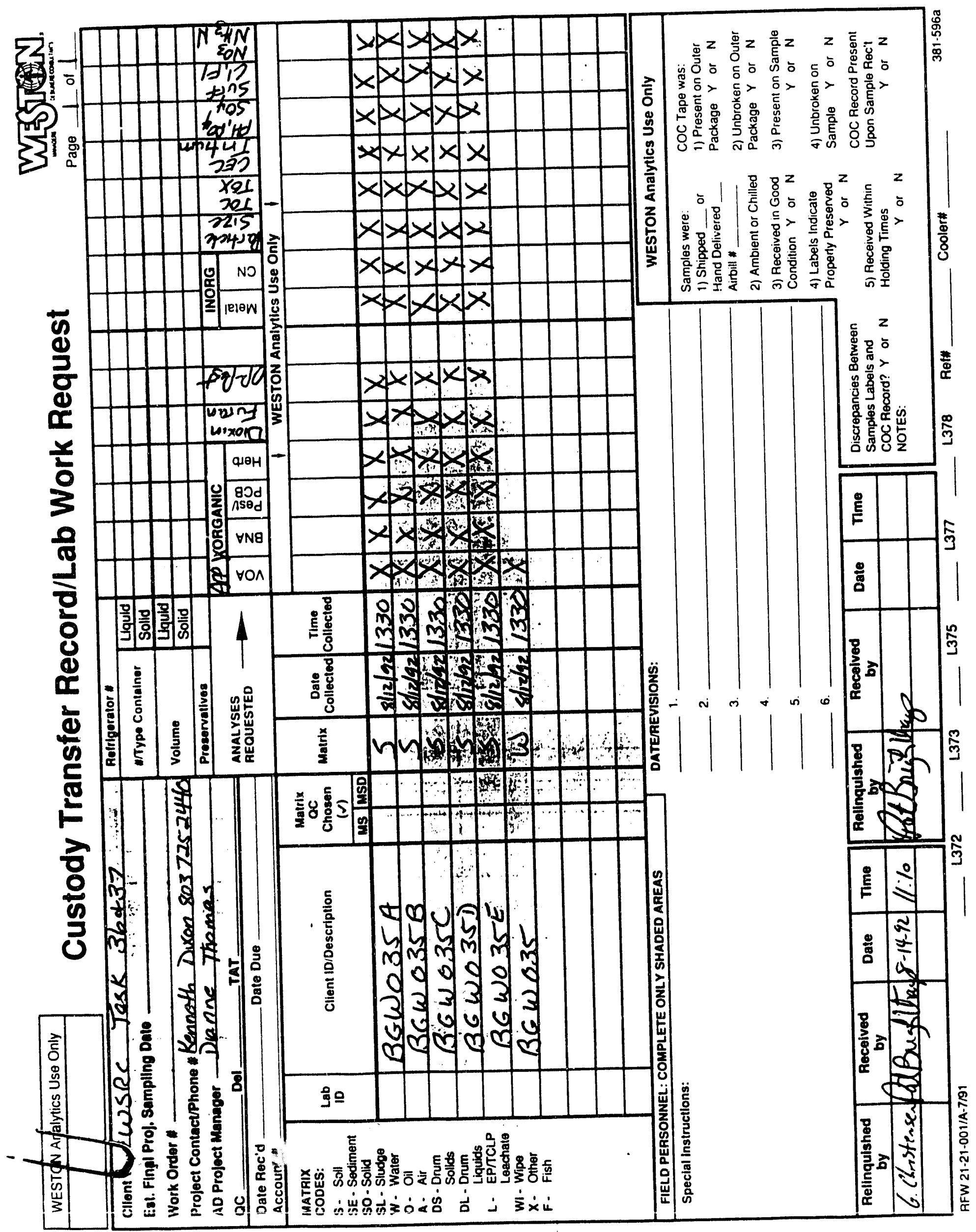




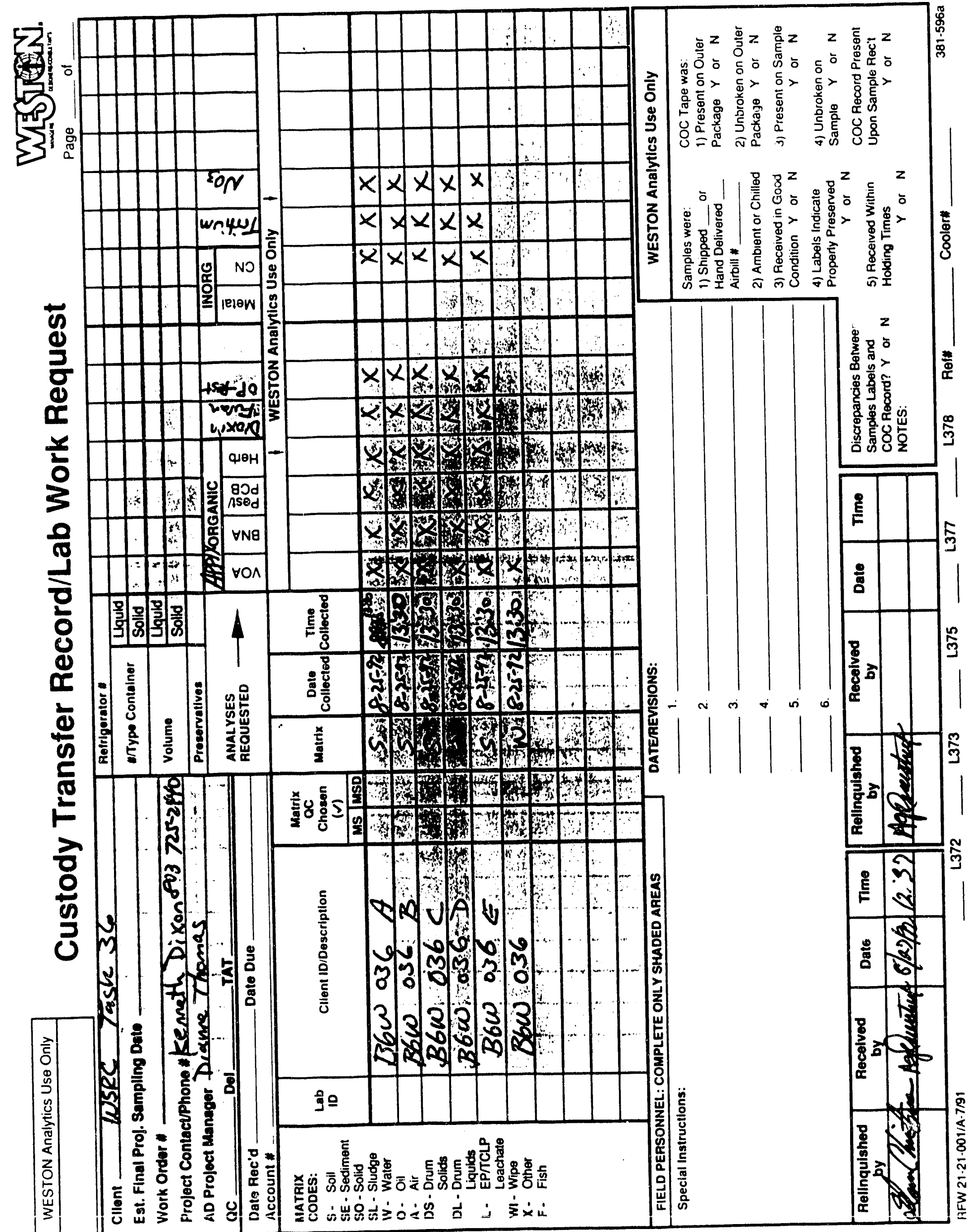




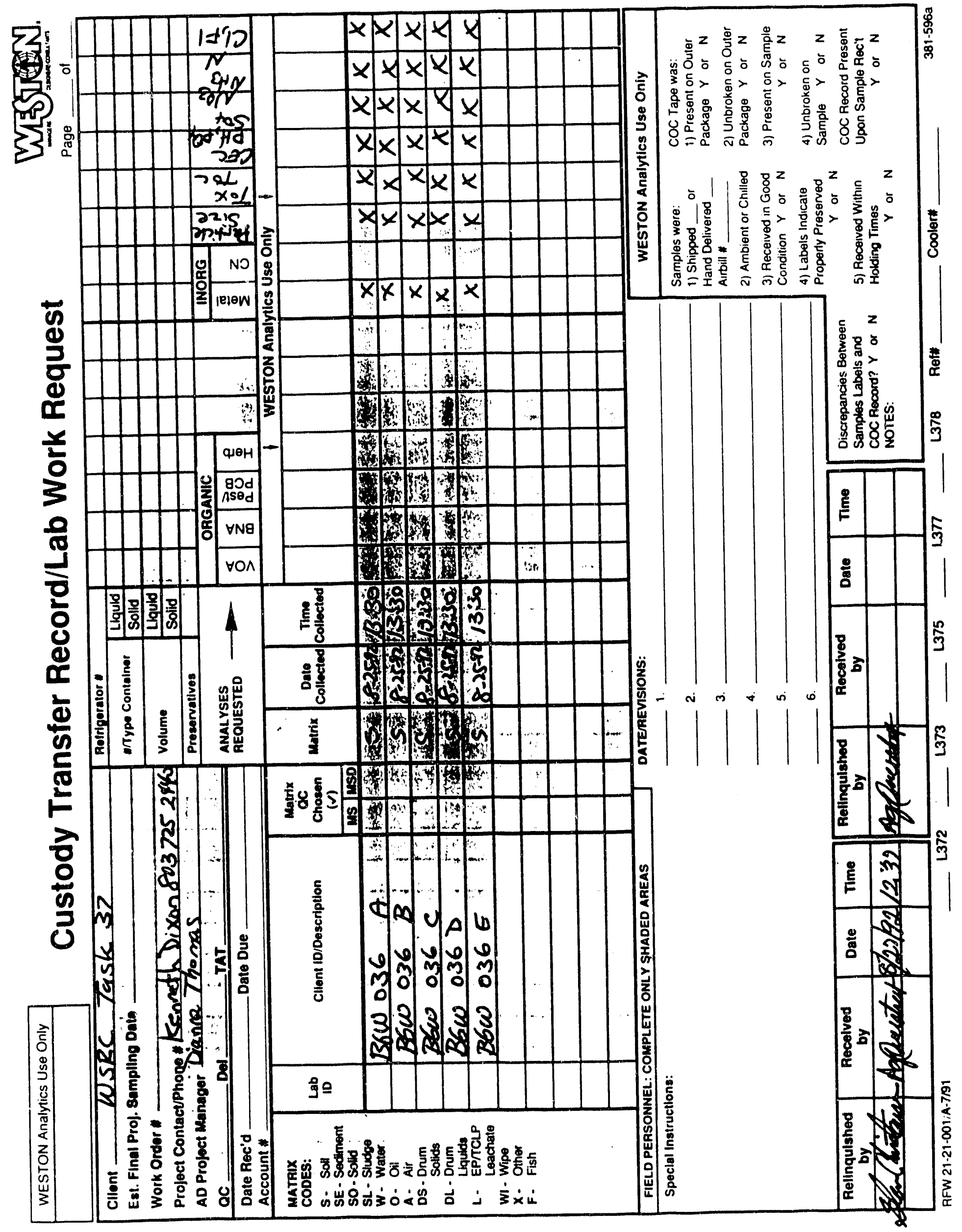




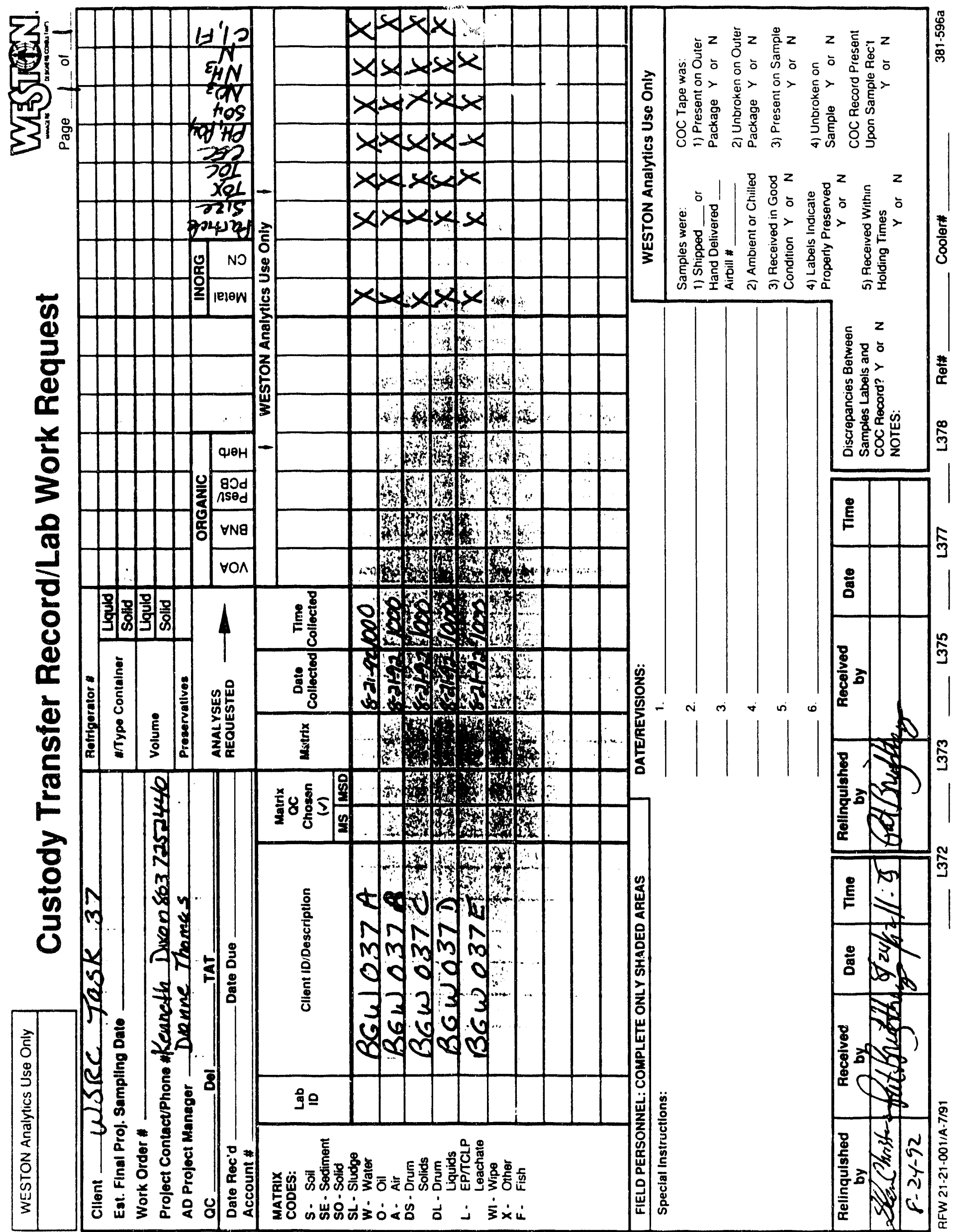




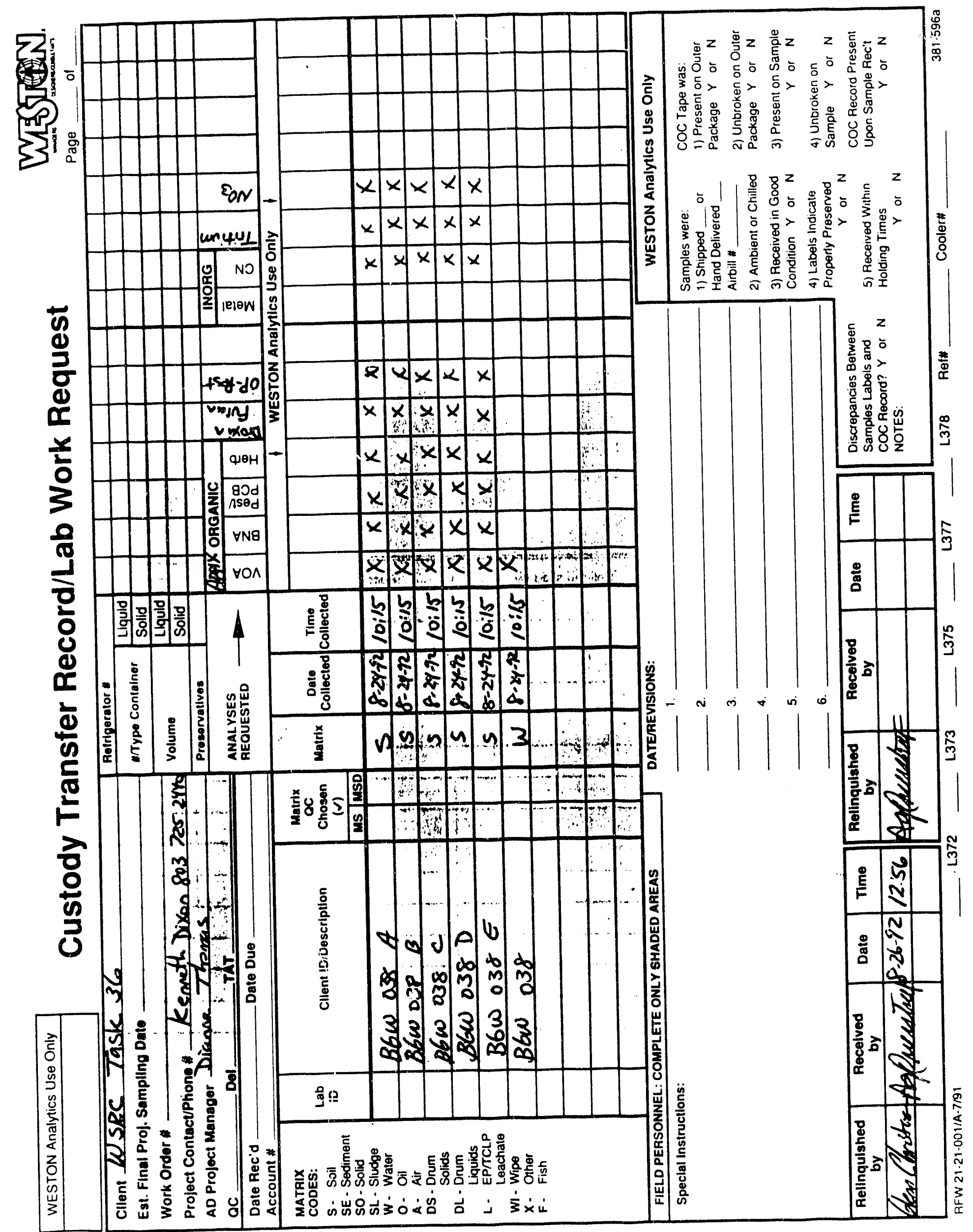




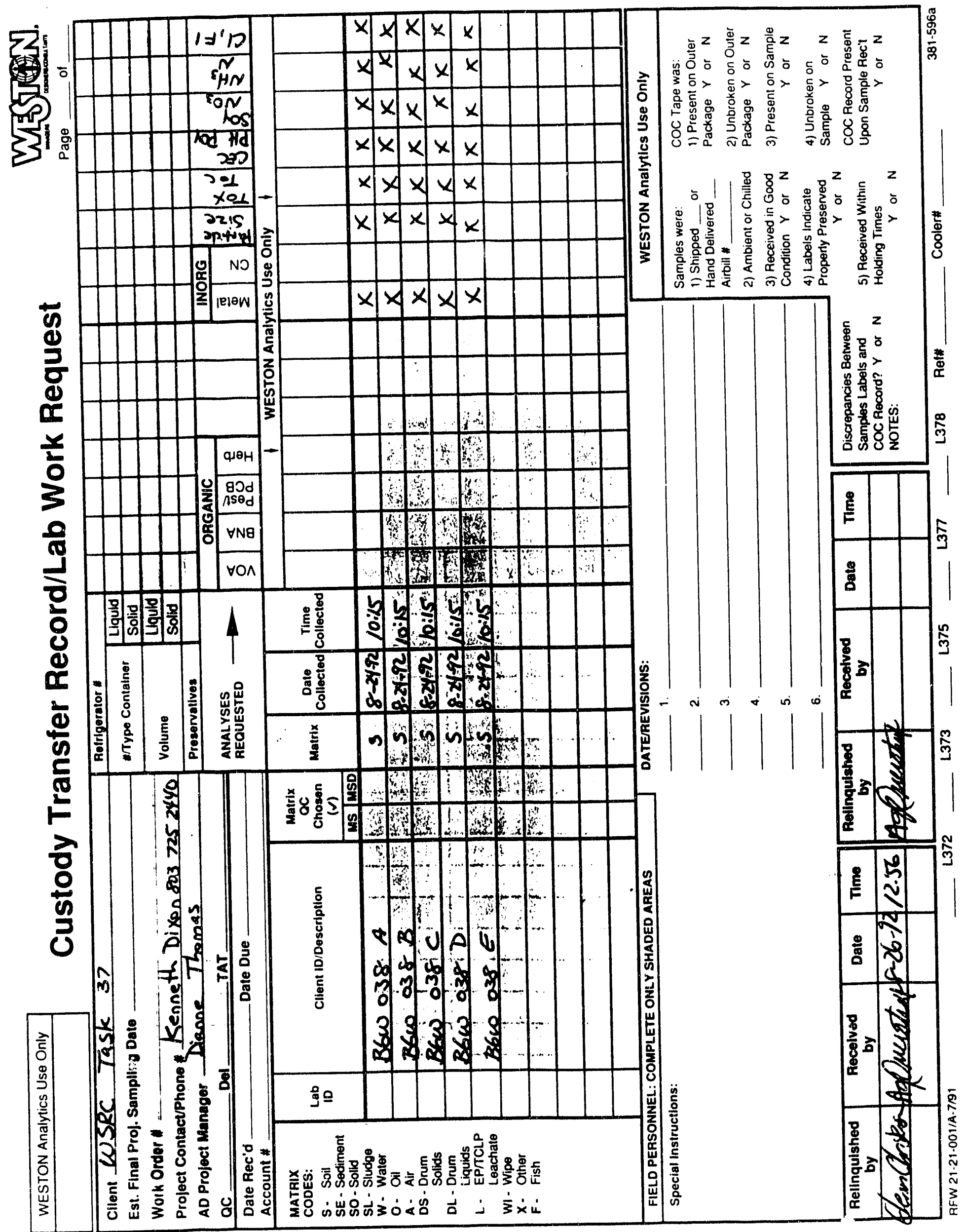




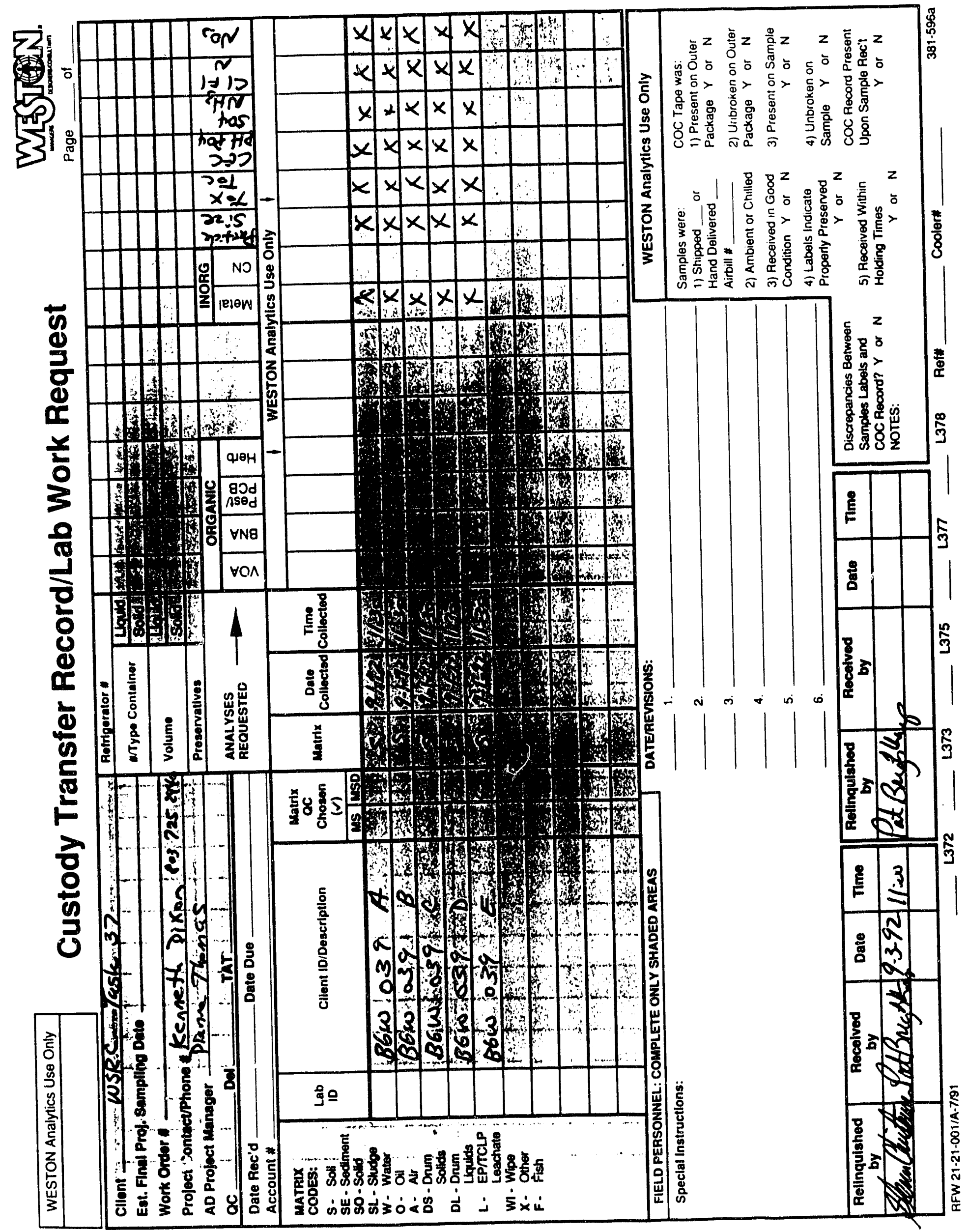




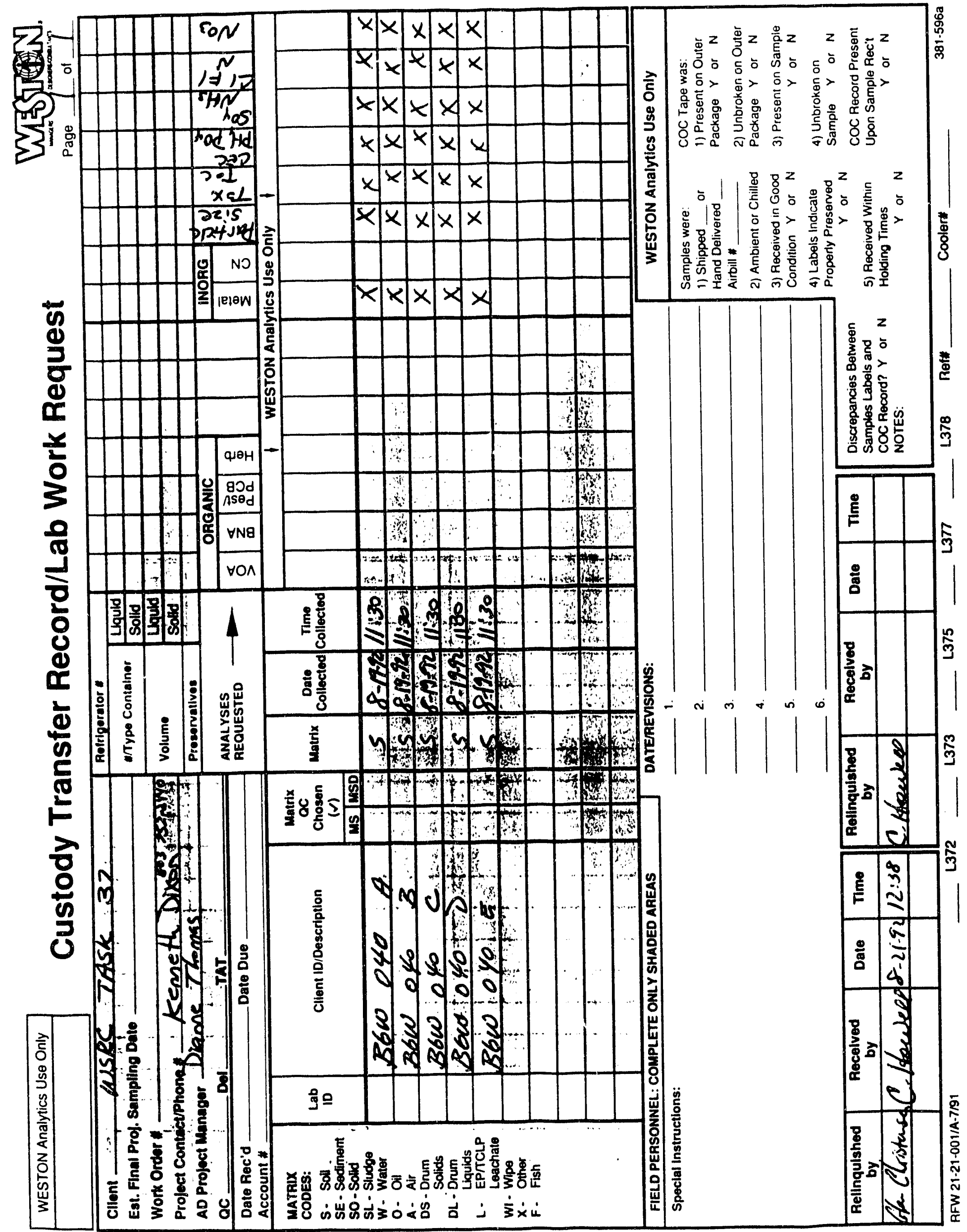




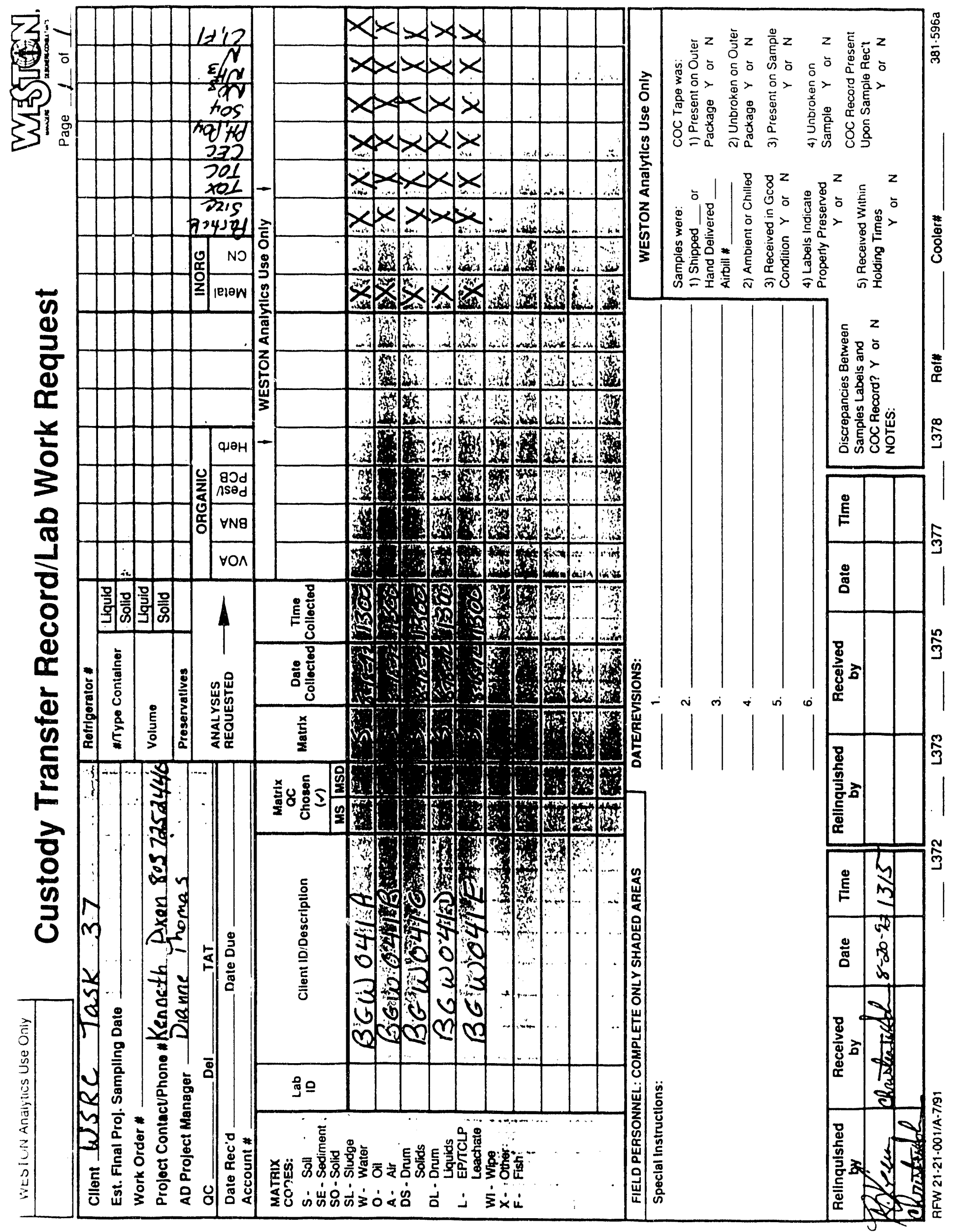




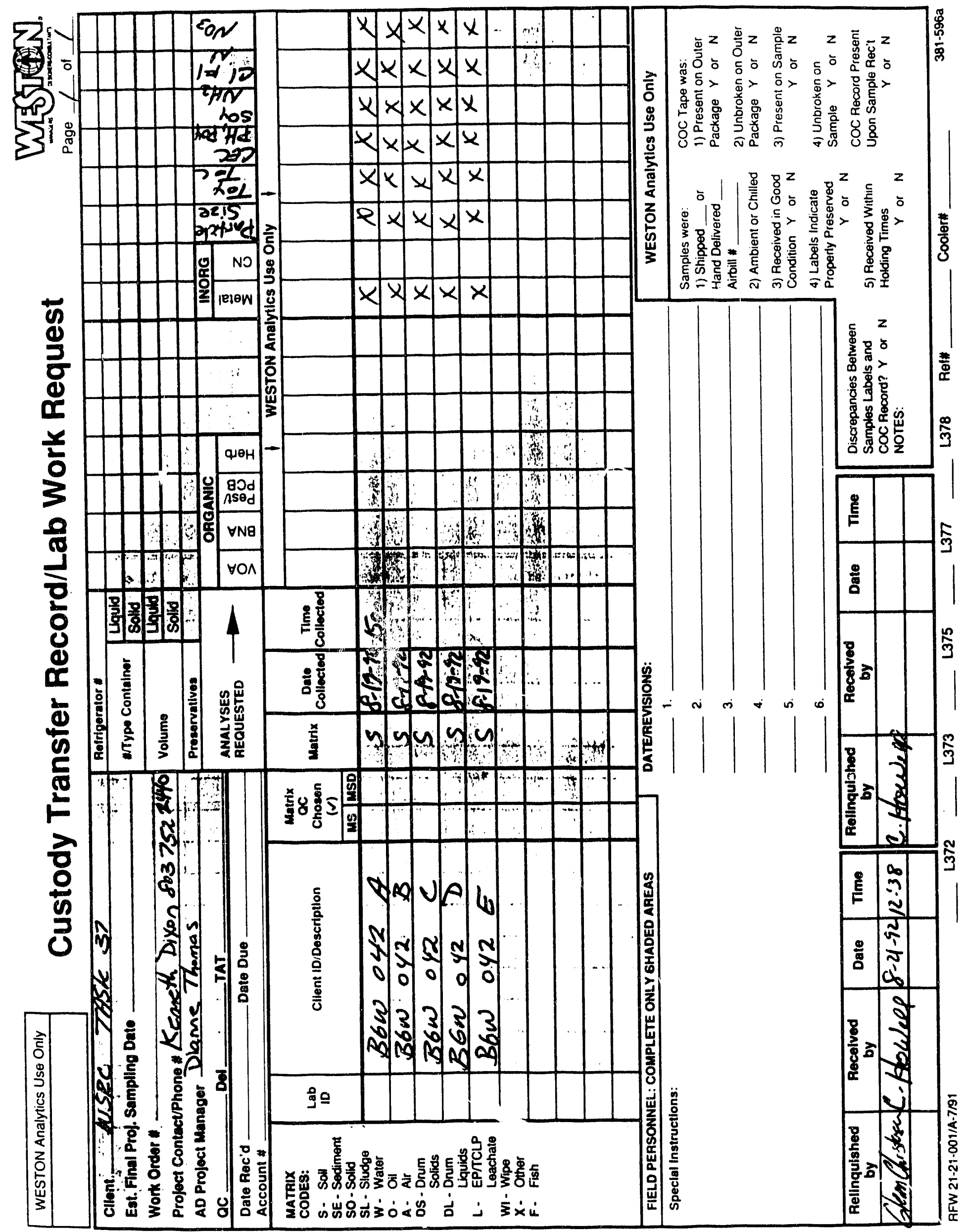




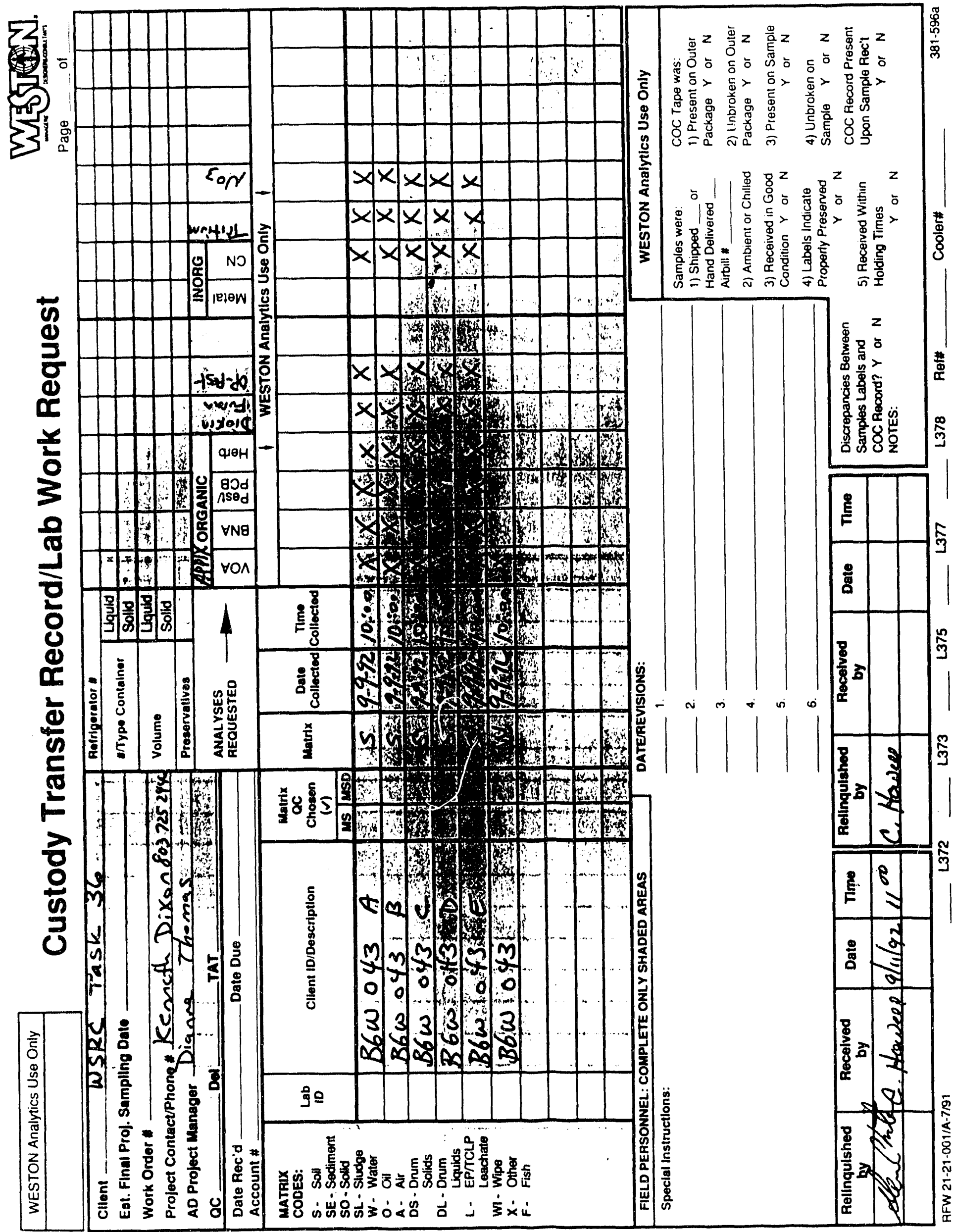




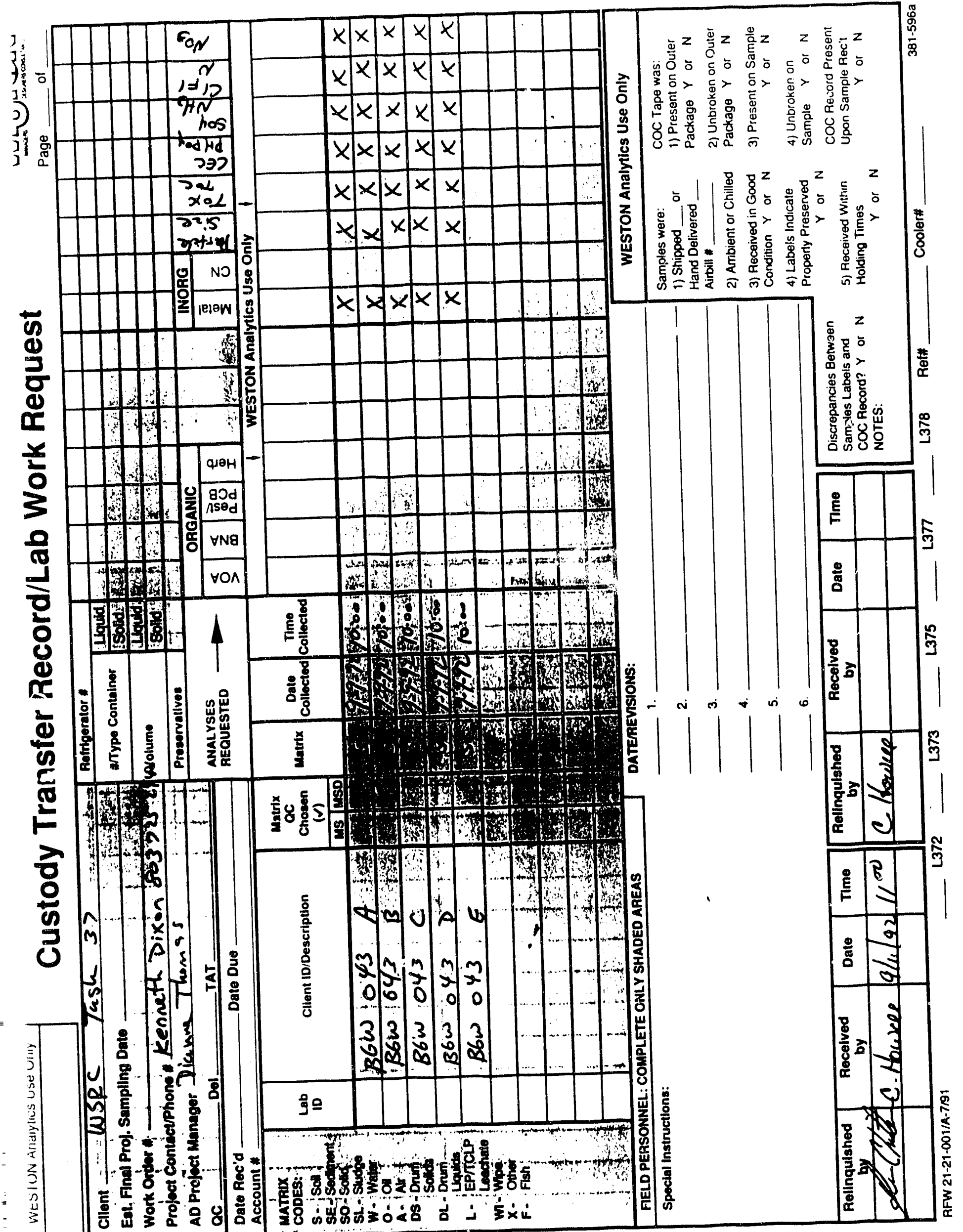




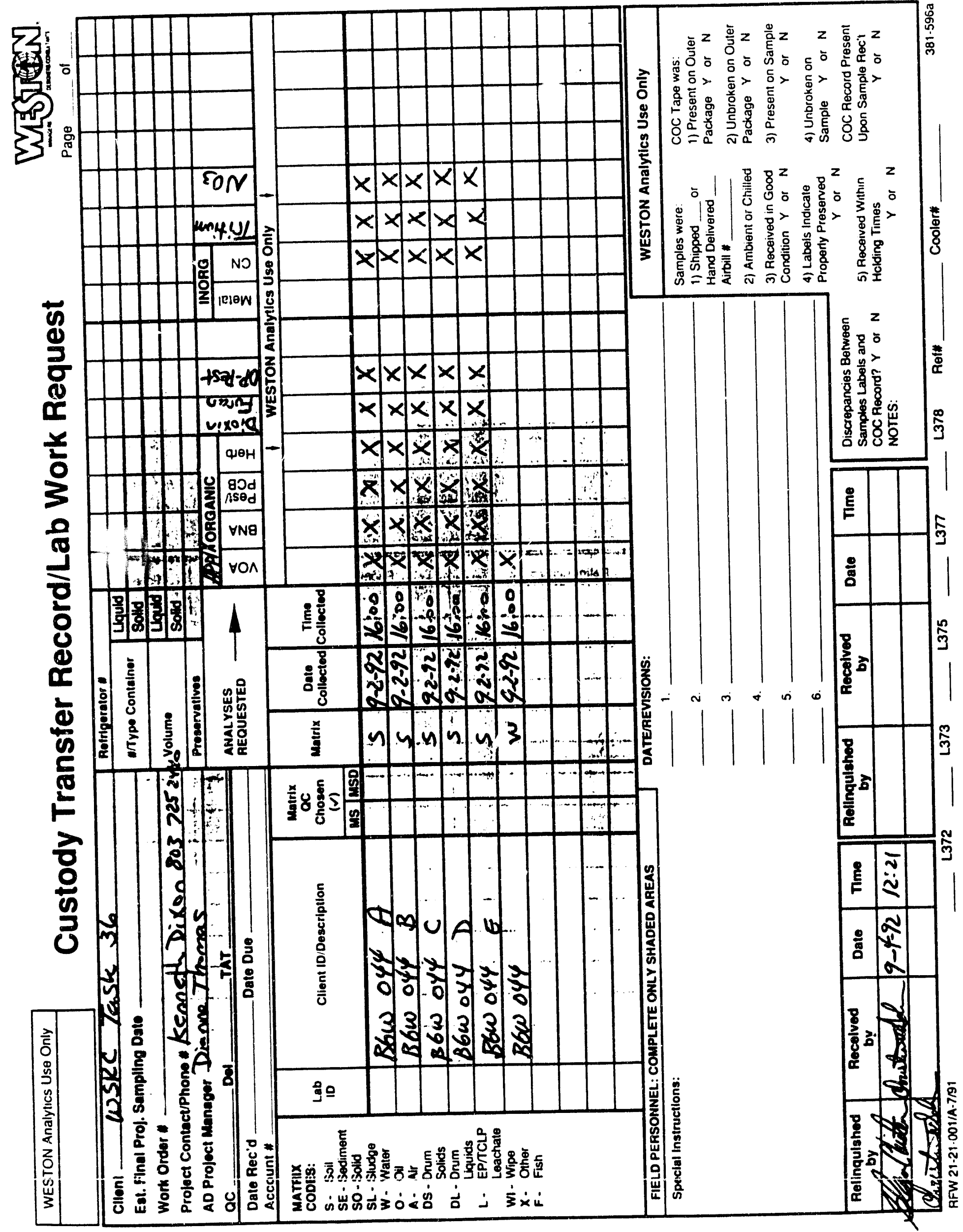


(3)

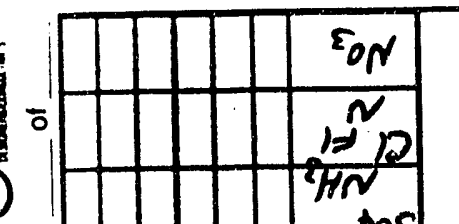

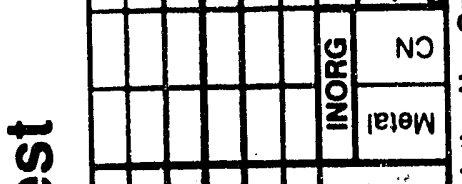

פ

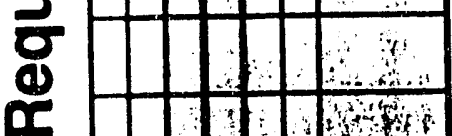

$\because$ L

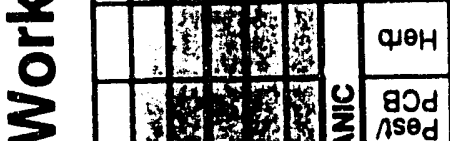

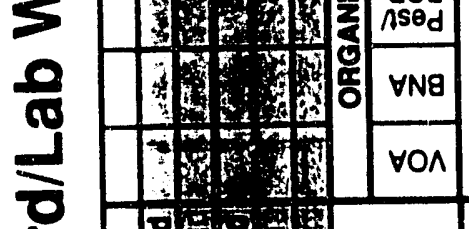

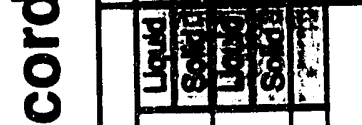

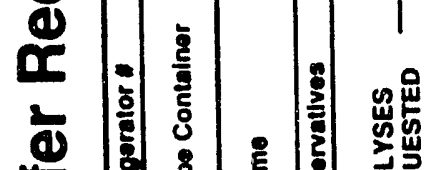

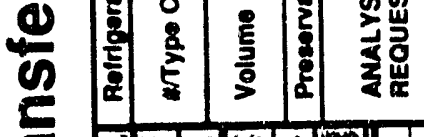

20 8

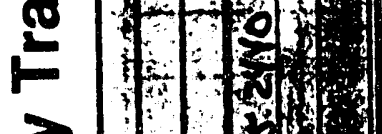

$\frac{3}{8}$ :

$\frac{9}{3} \sqrt{3} \cdot \sqrt{3}$

0

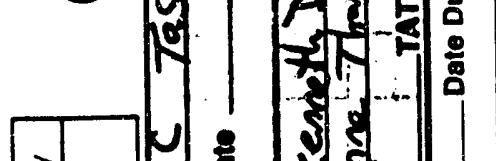

글

兽

鸟

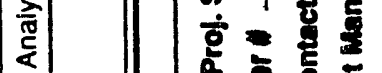

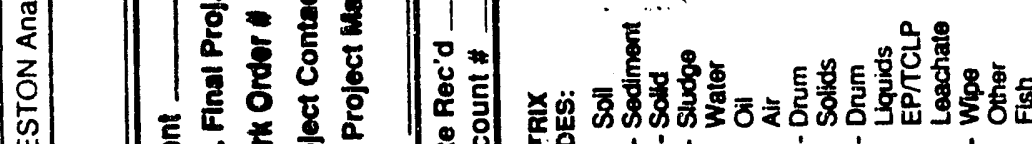

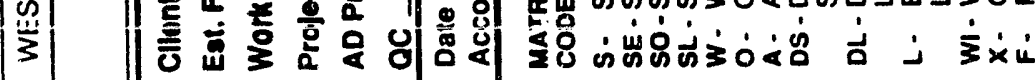

-4 40 क

$8 \mid 0.906$

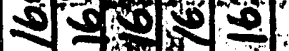

$\div \frac{8}{4}$ 娄

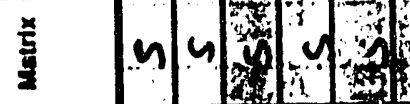

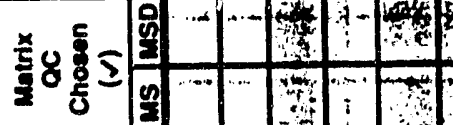

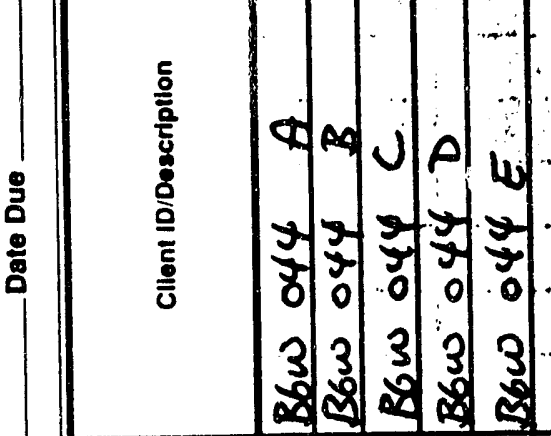

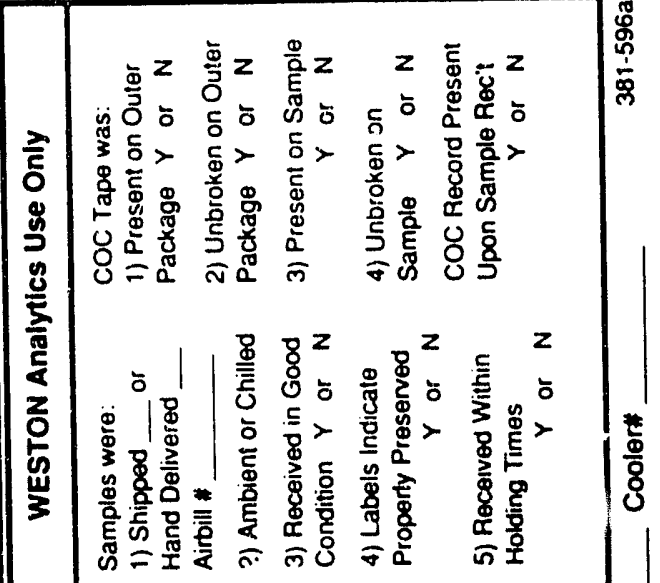

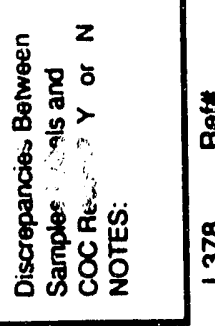

롵

10

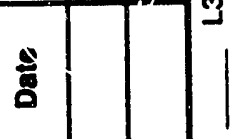

8

$\frac{8}{8}$

罂

i $\infty+\infty$

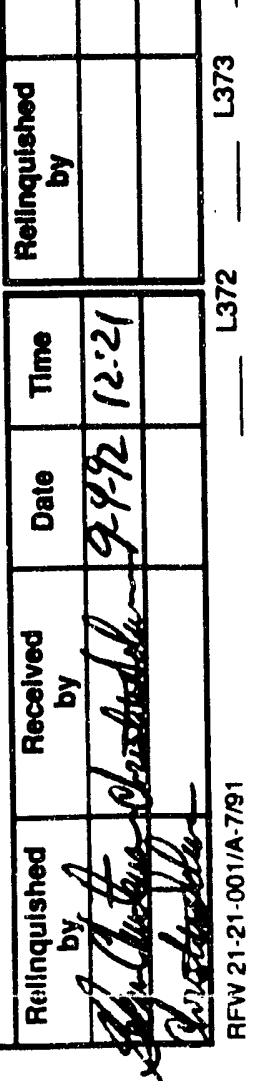




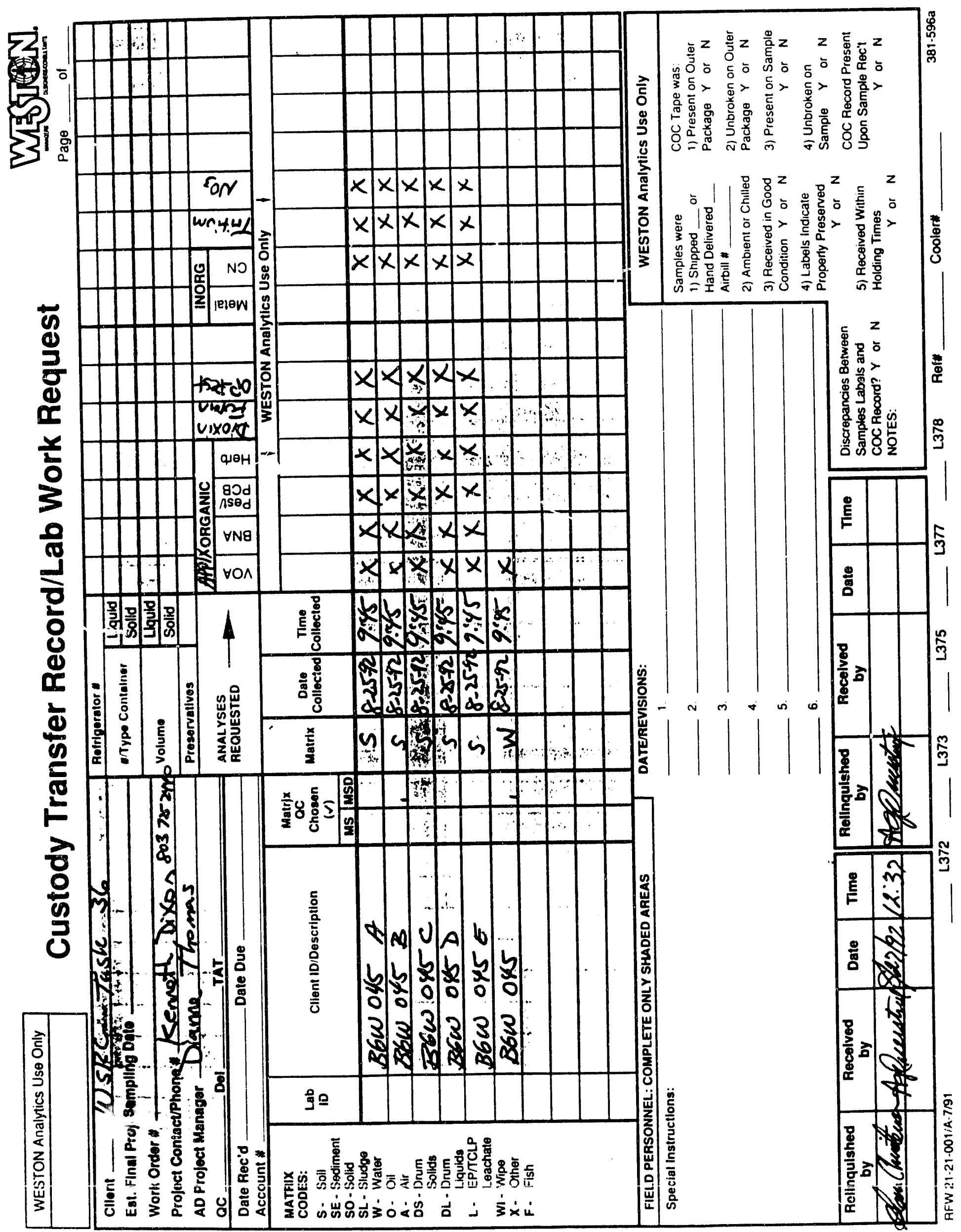




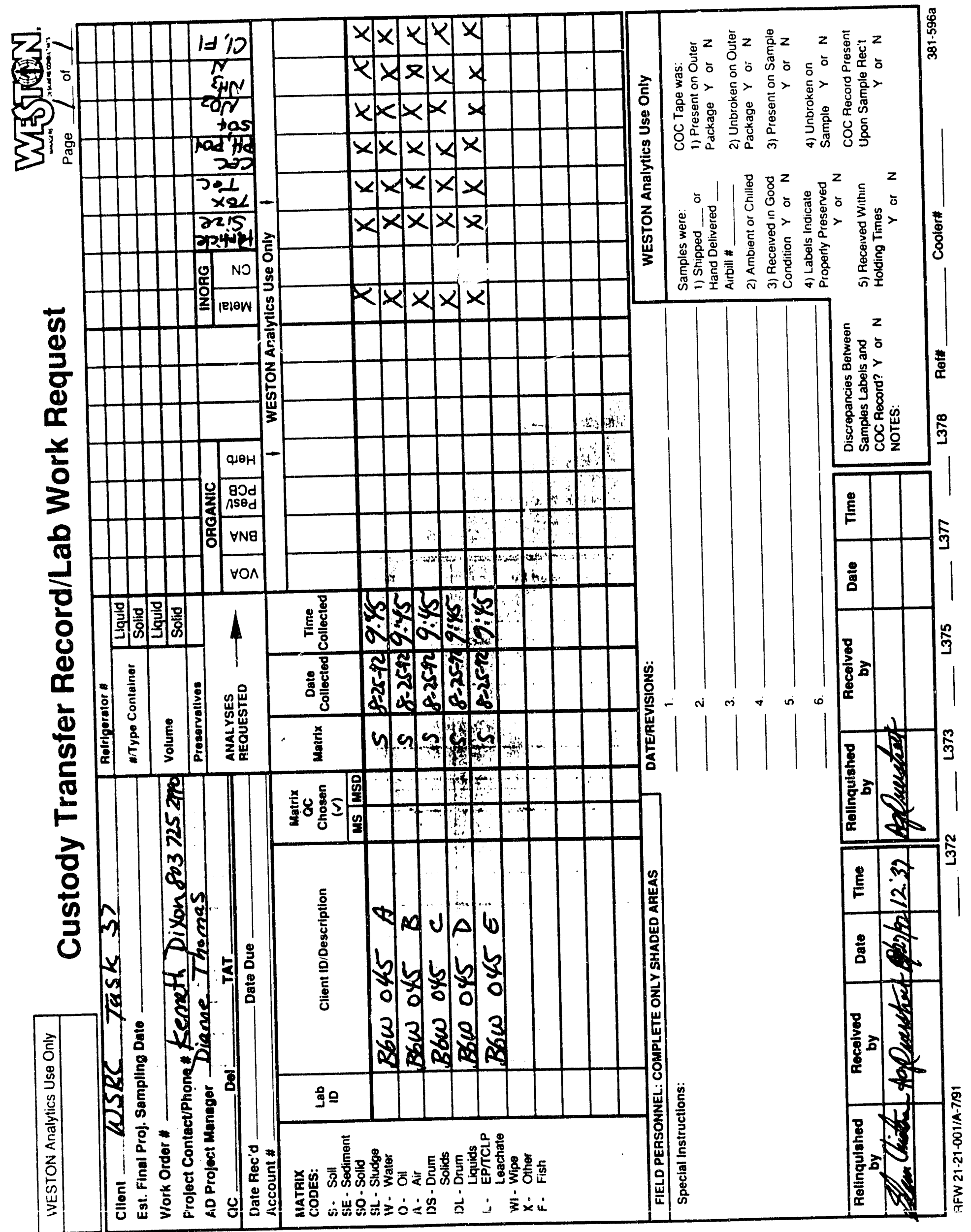




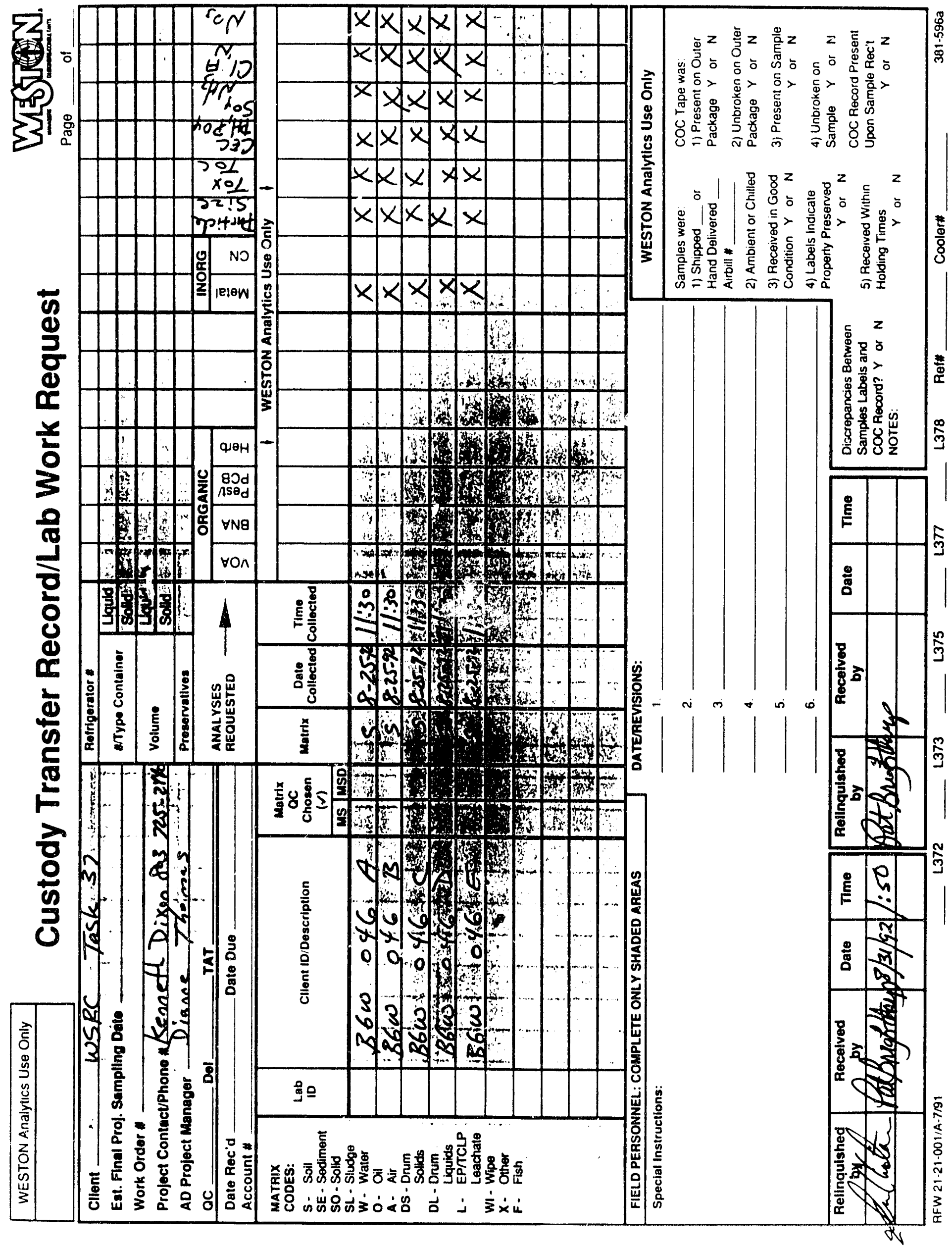




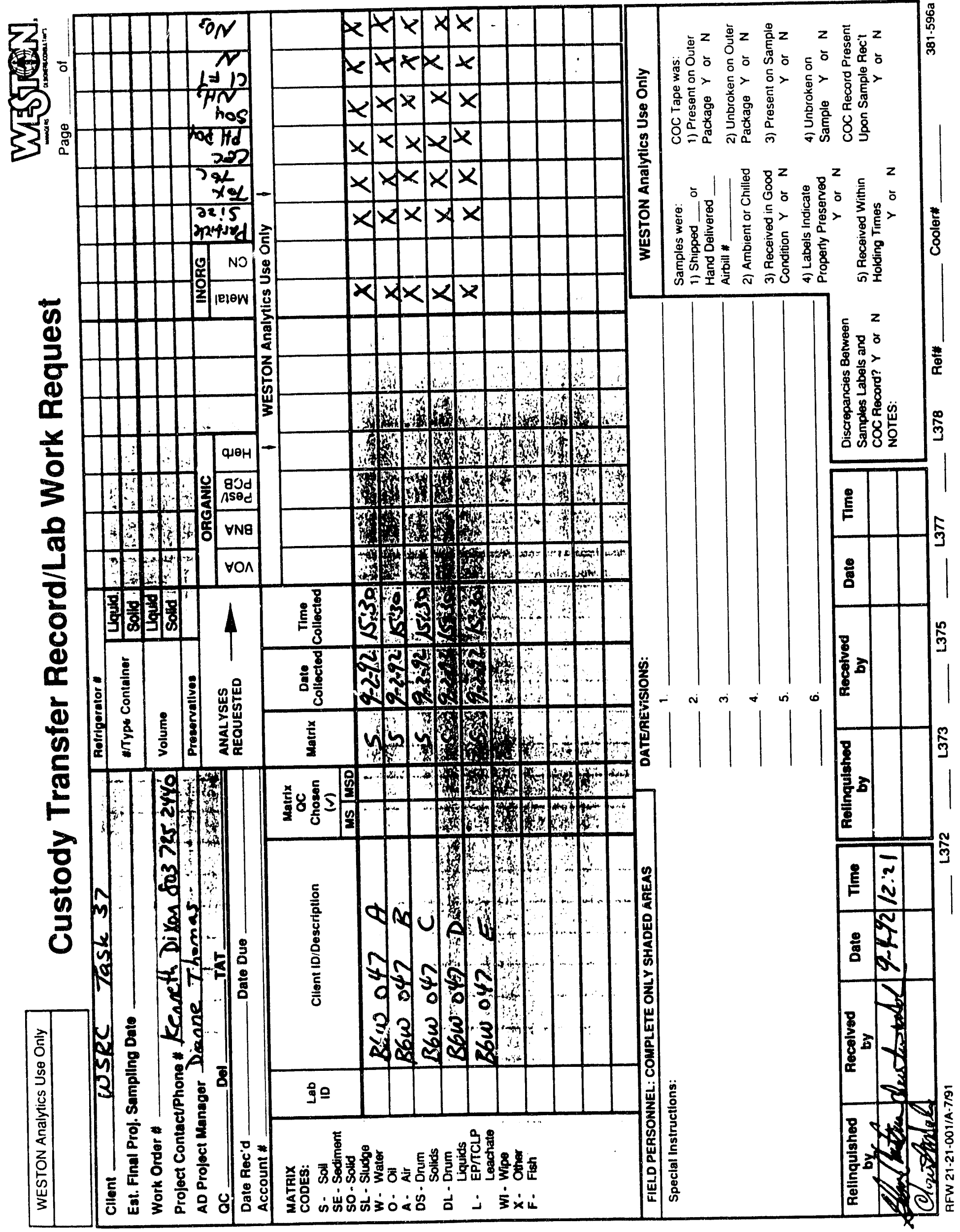




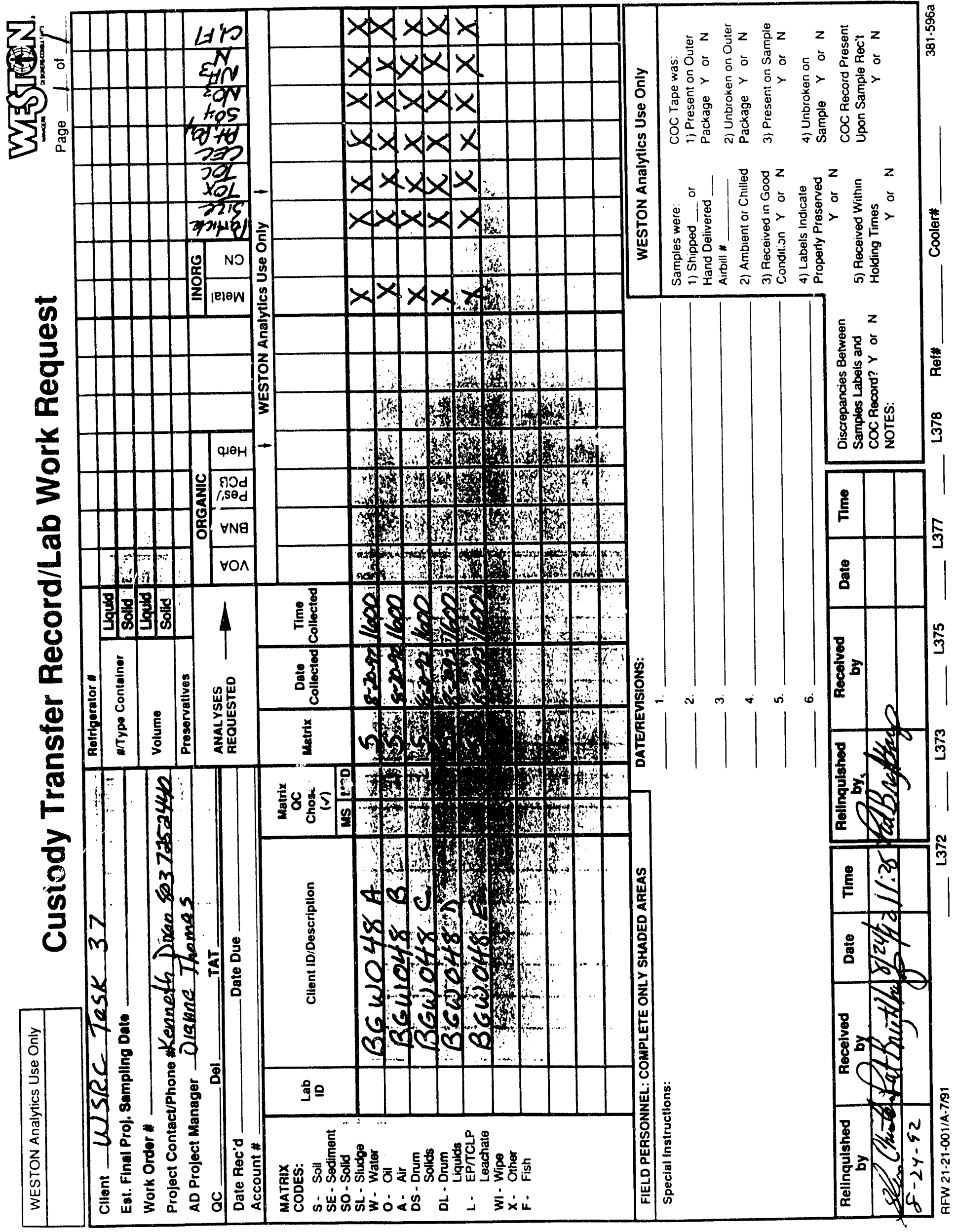




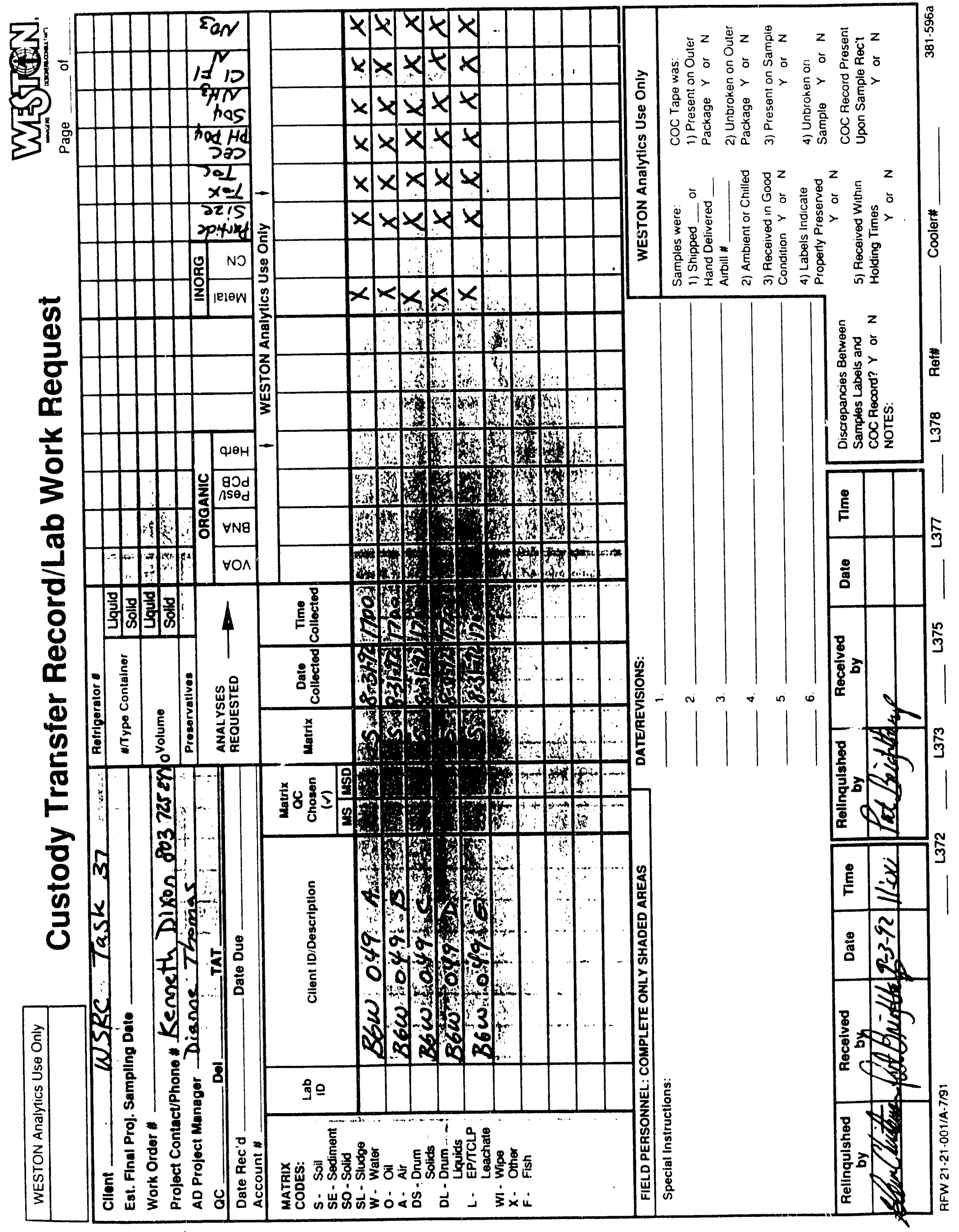




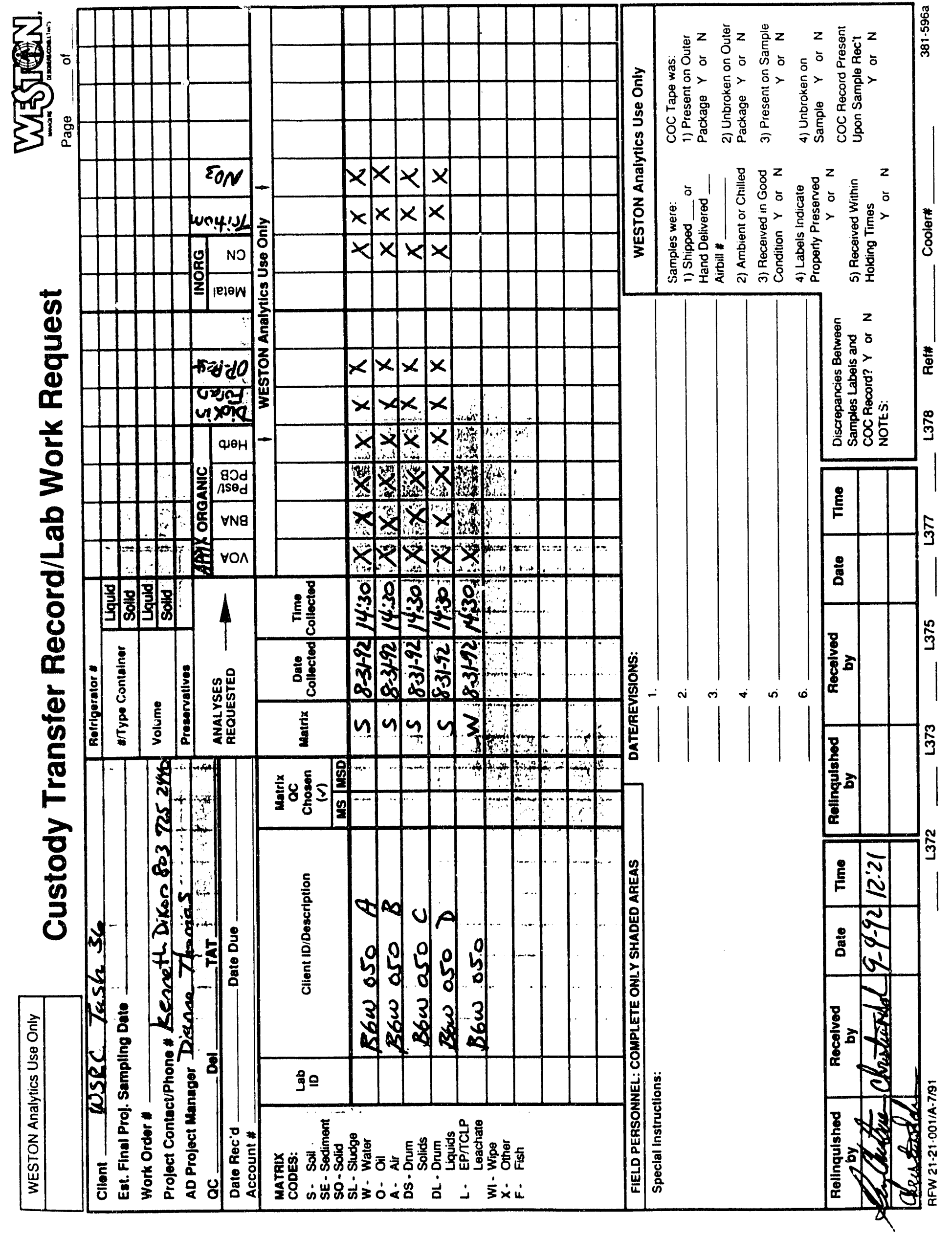




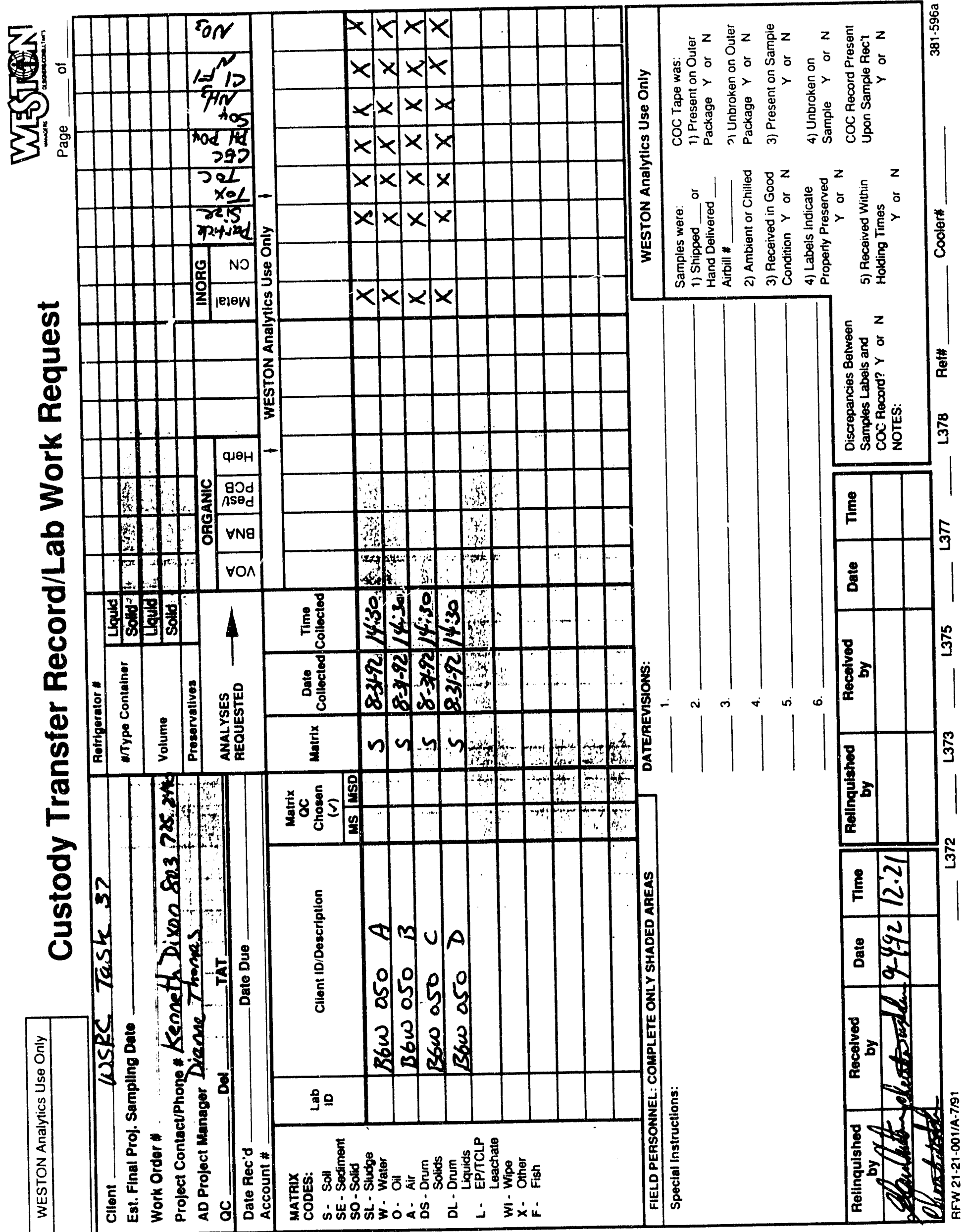




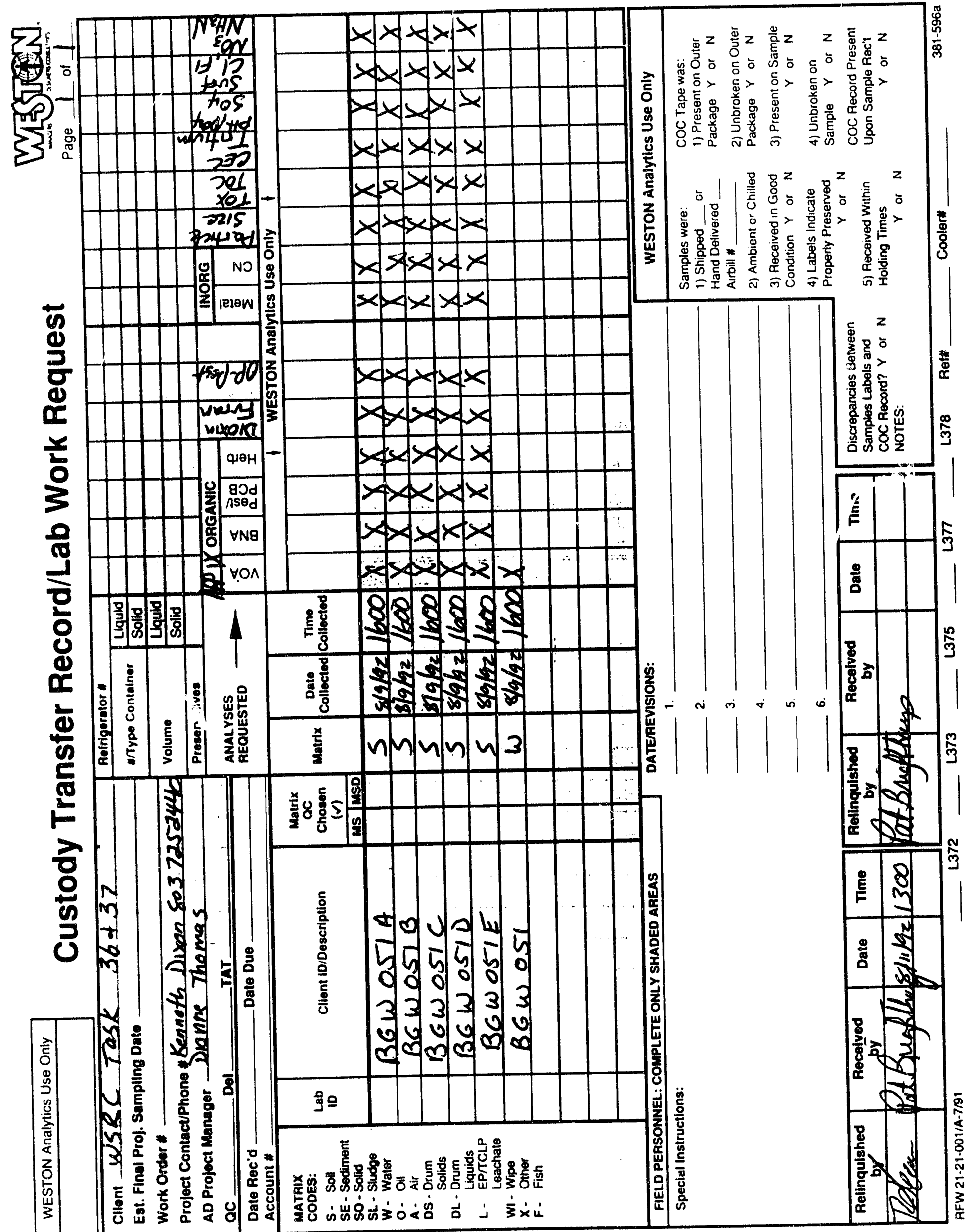




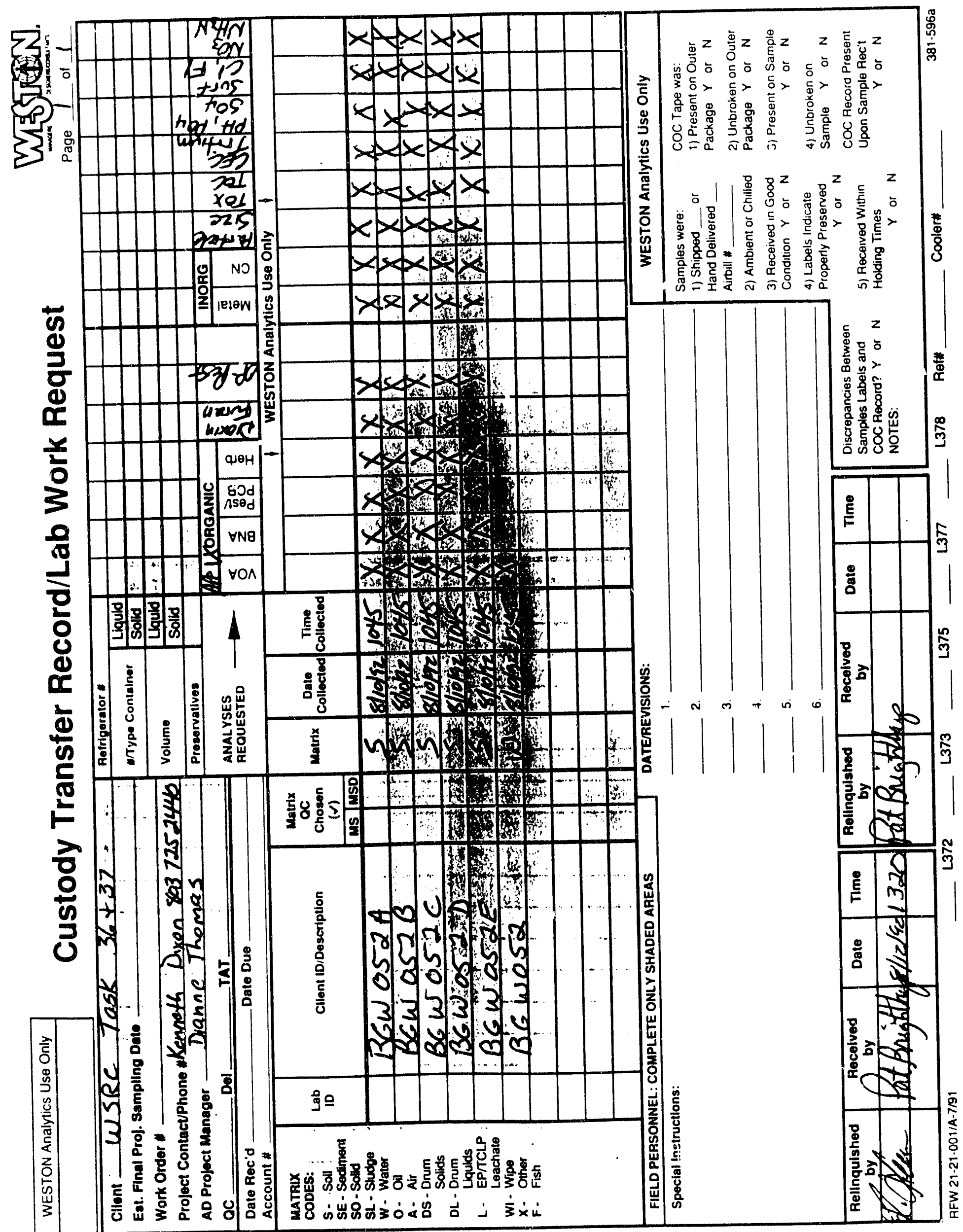




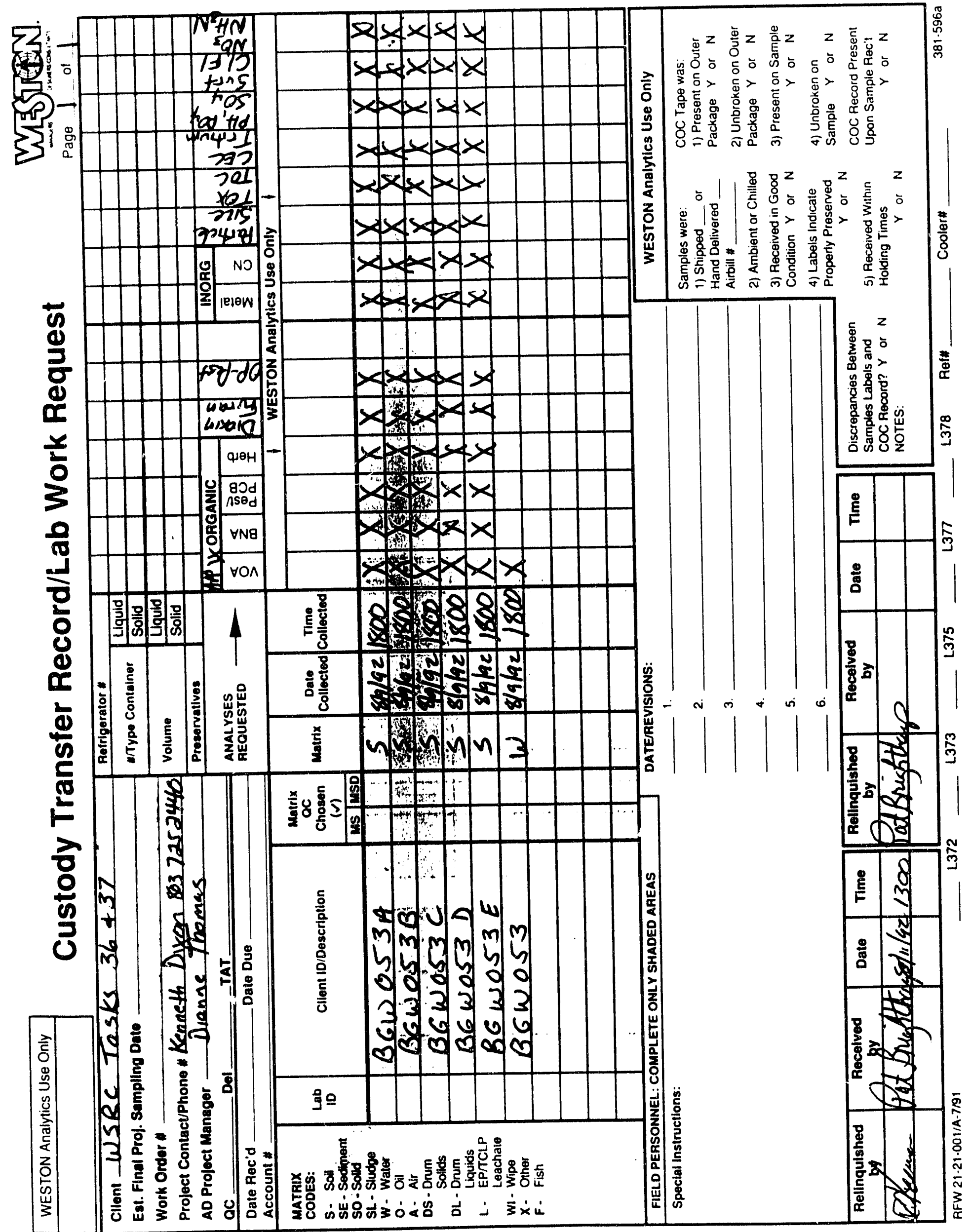




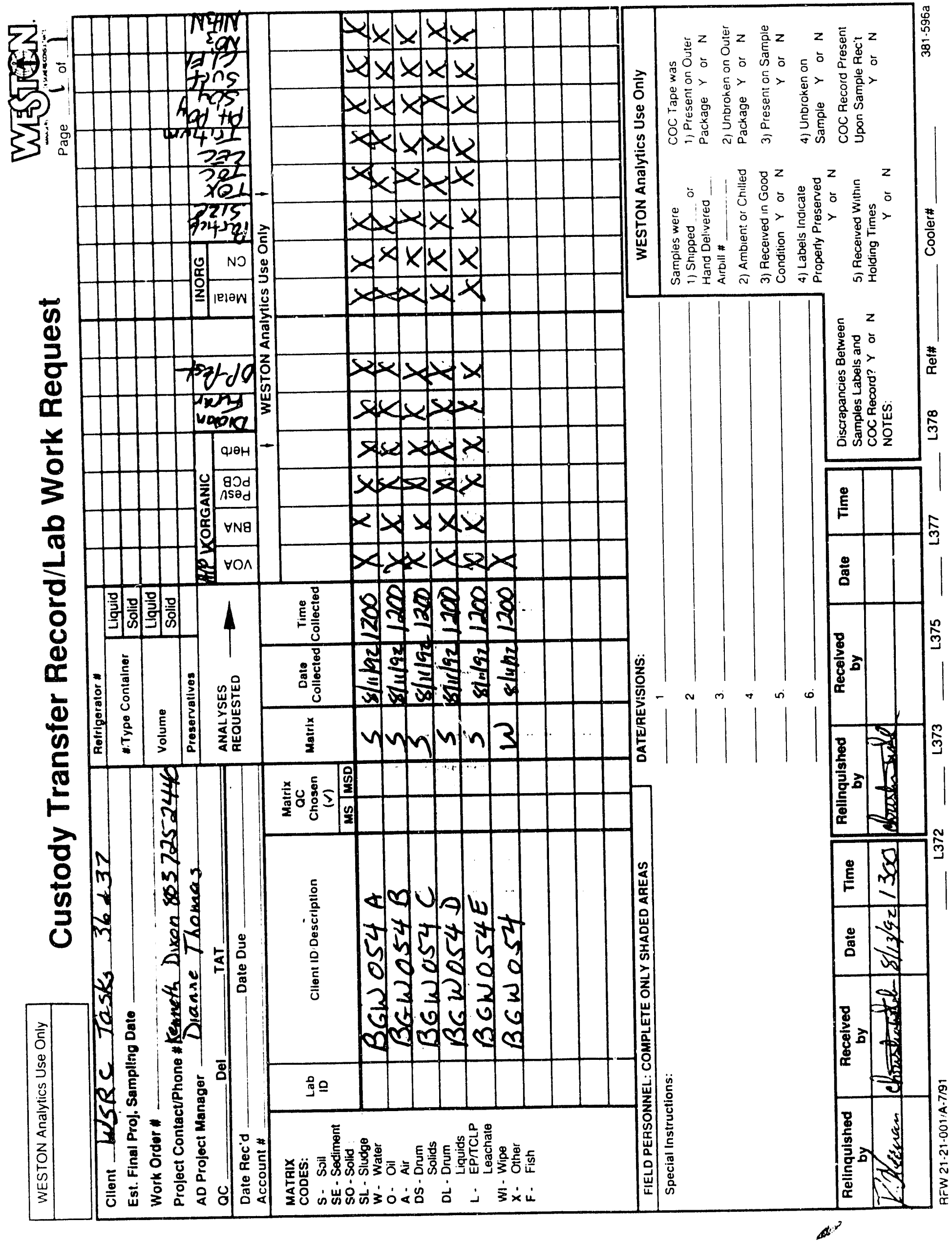




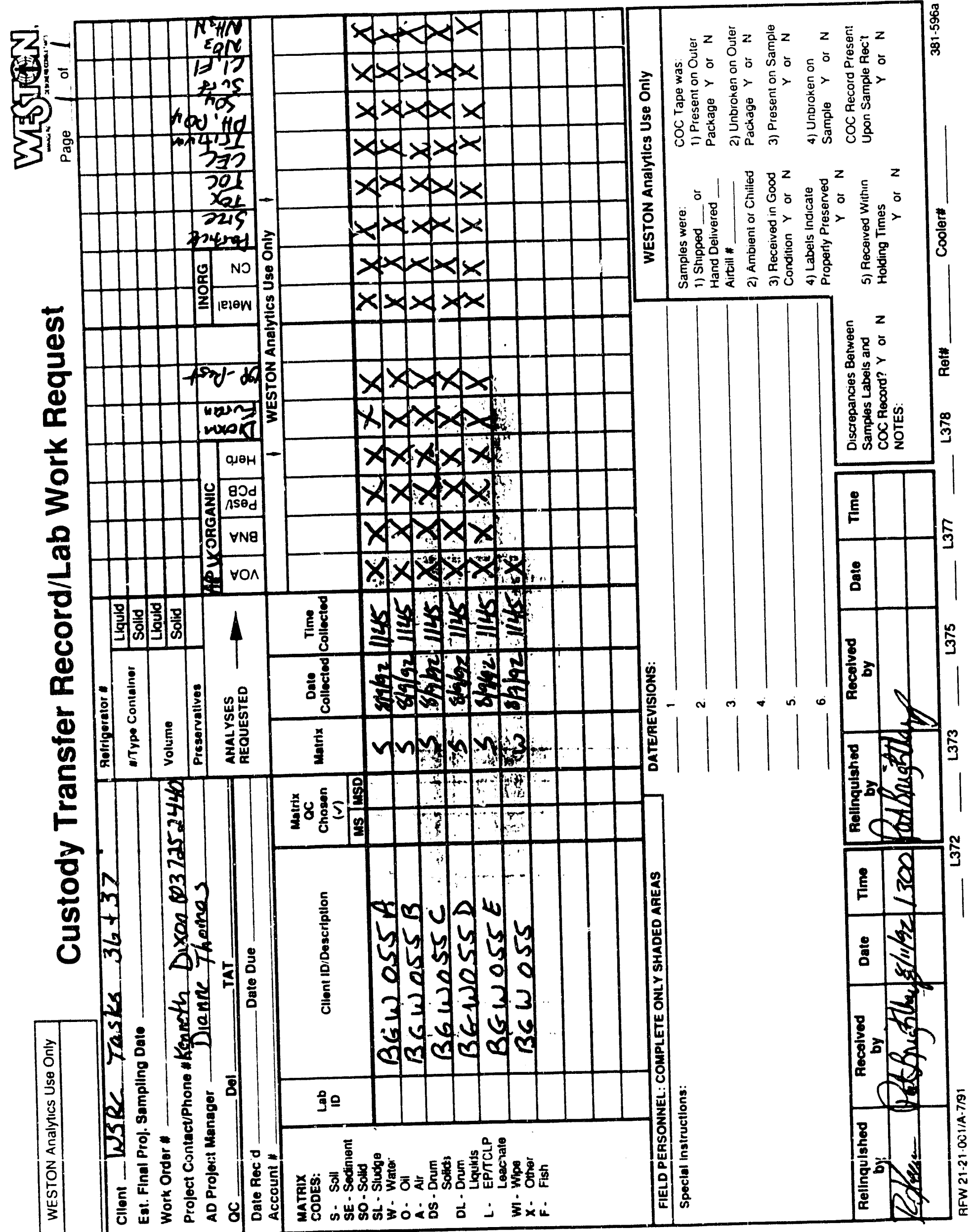




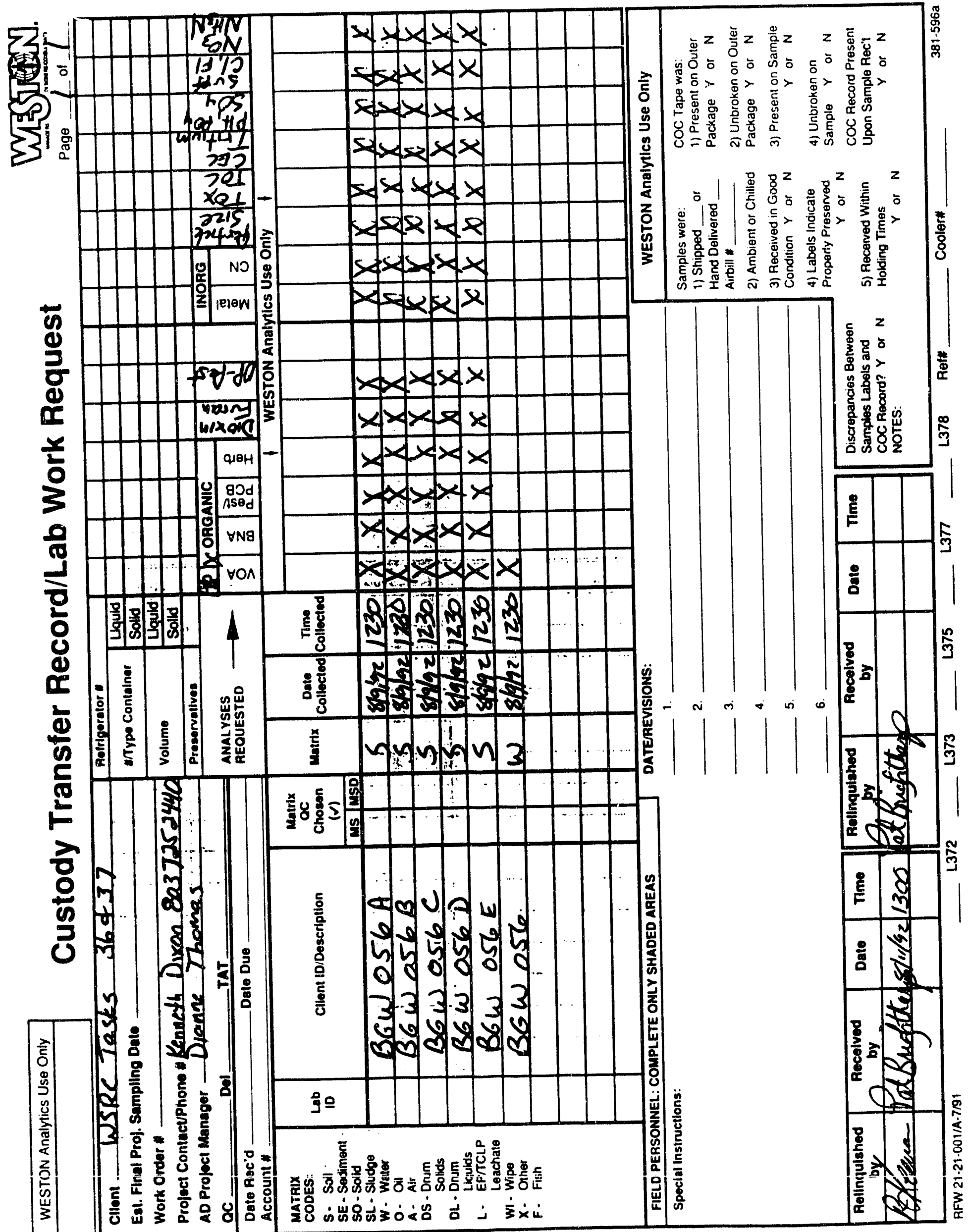




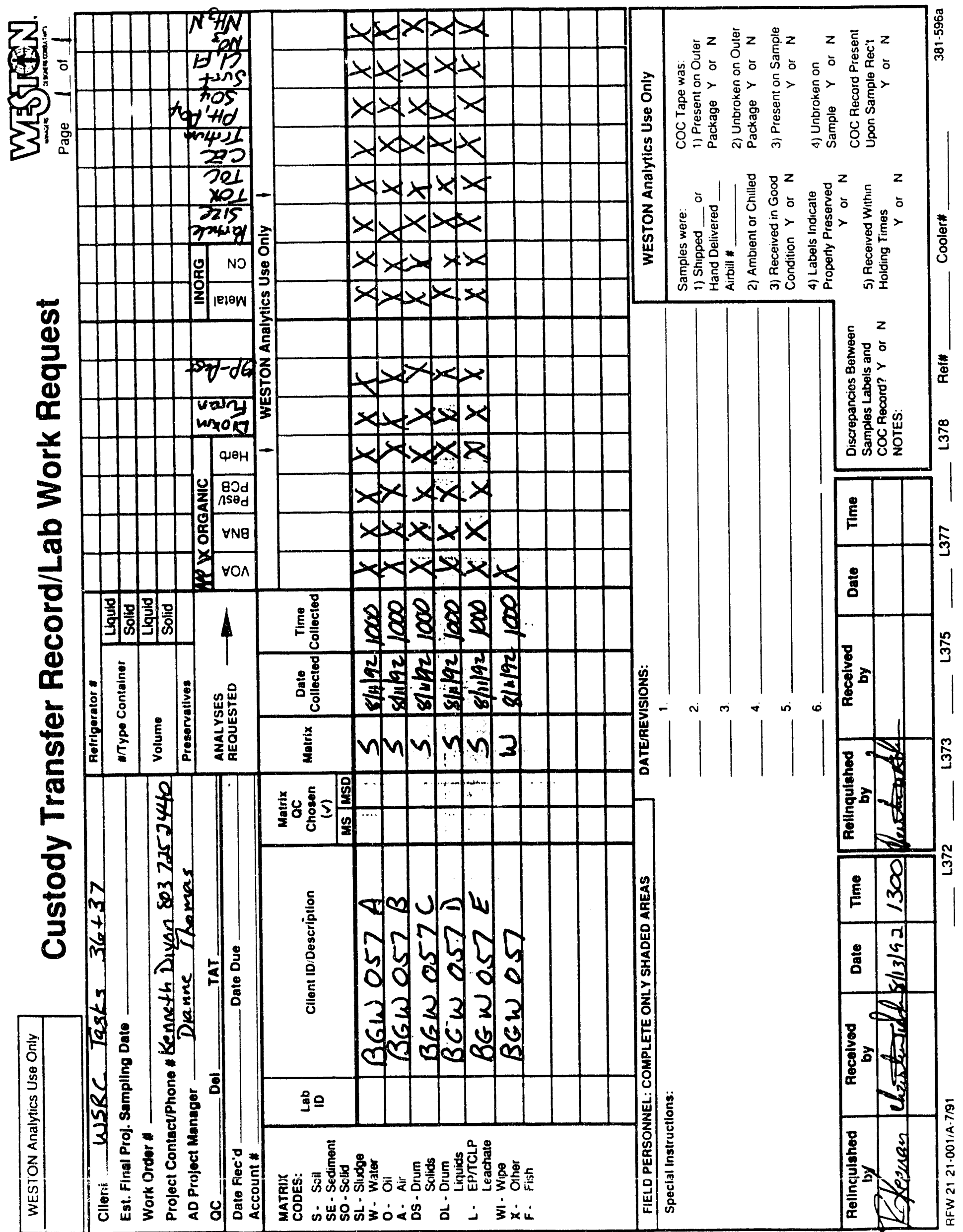




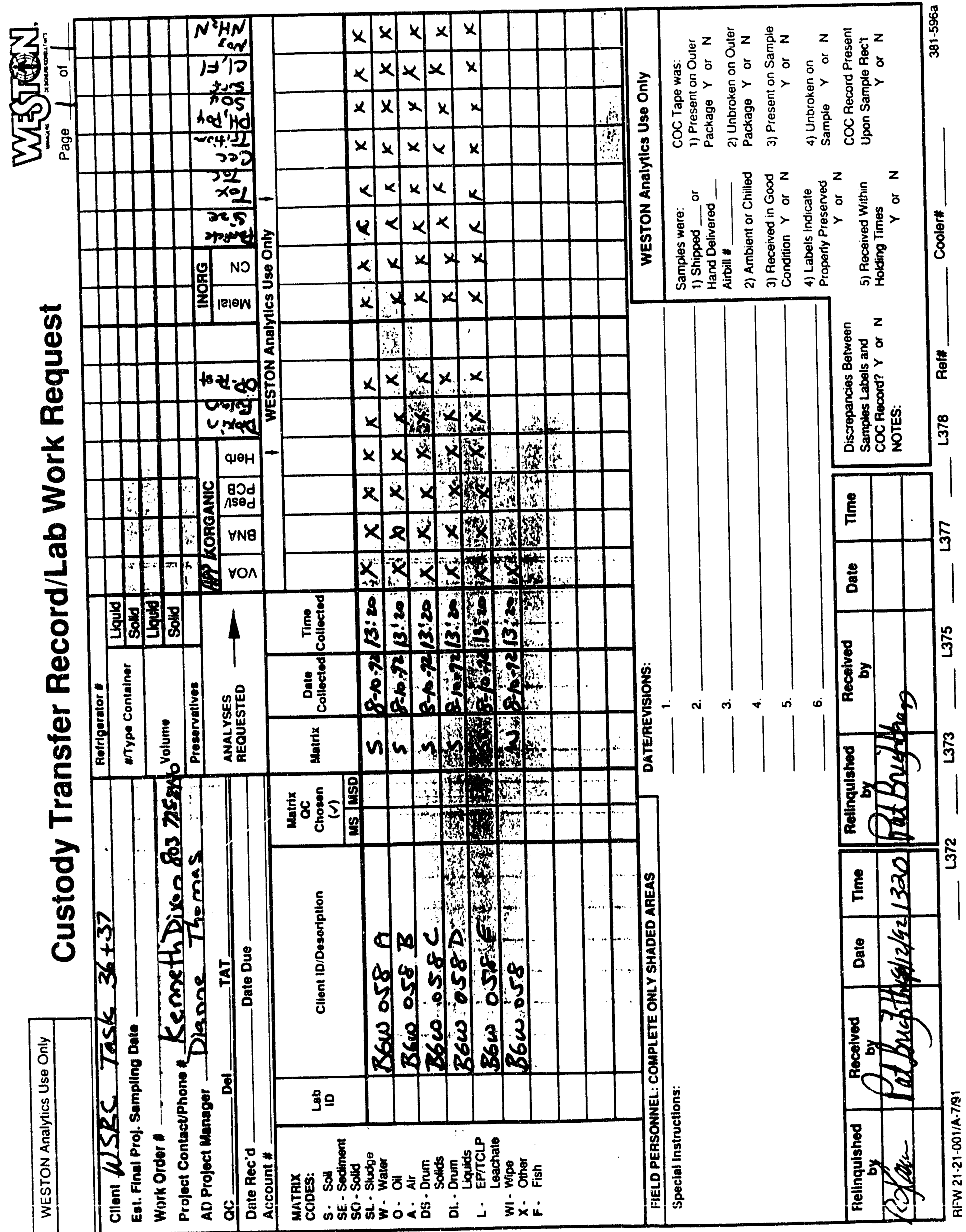




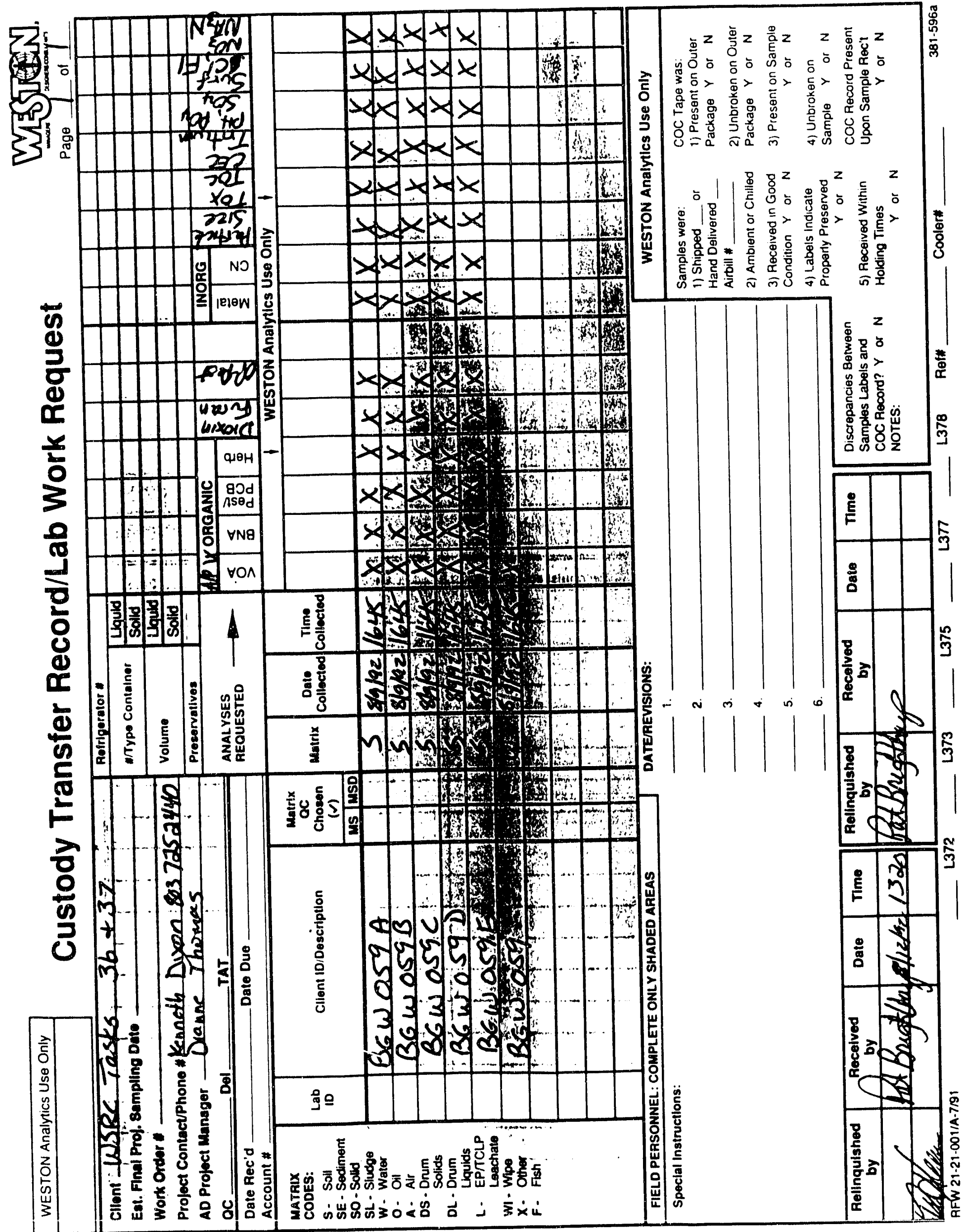




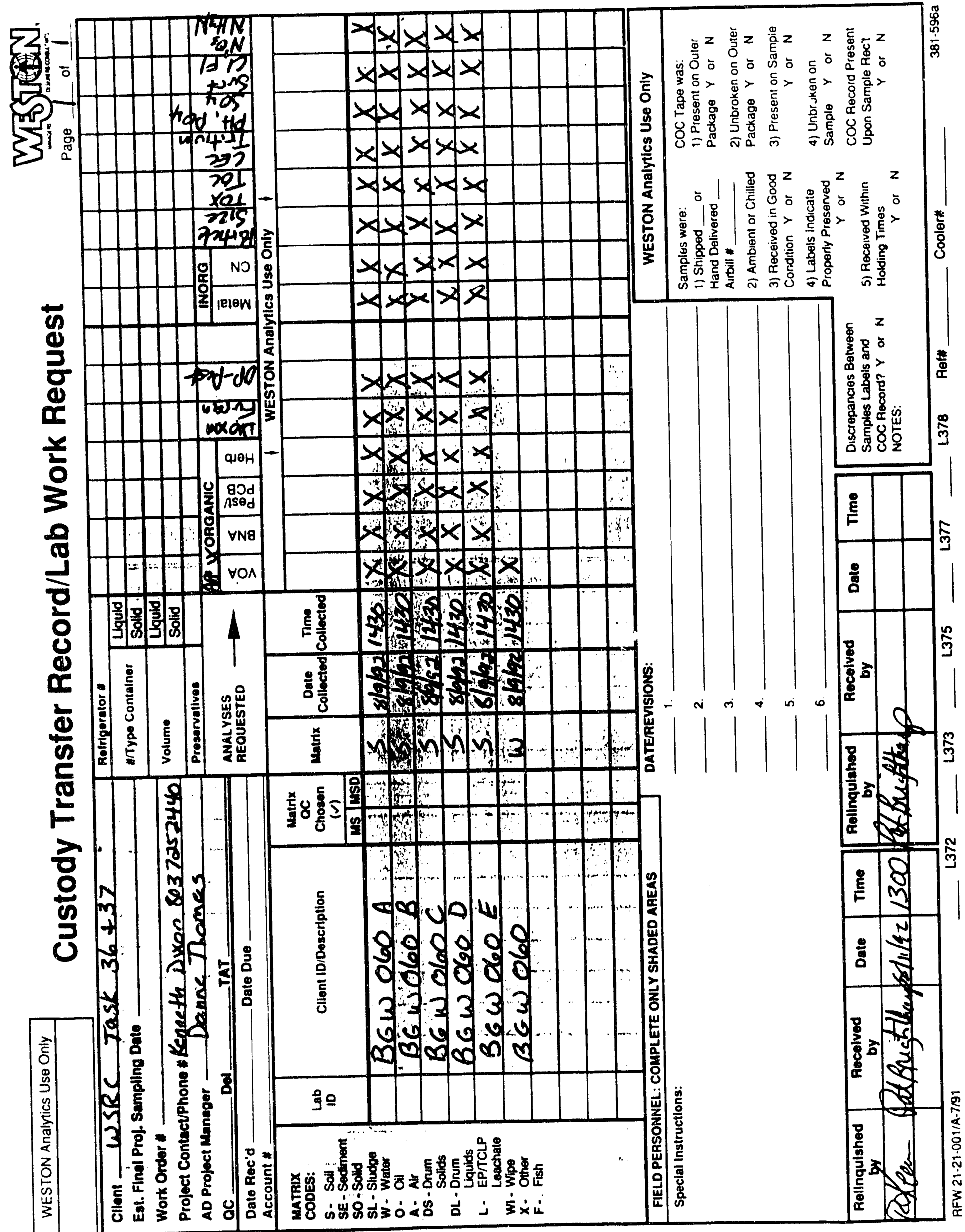




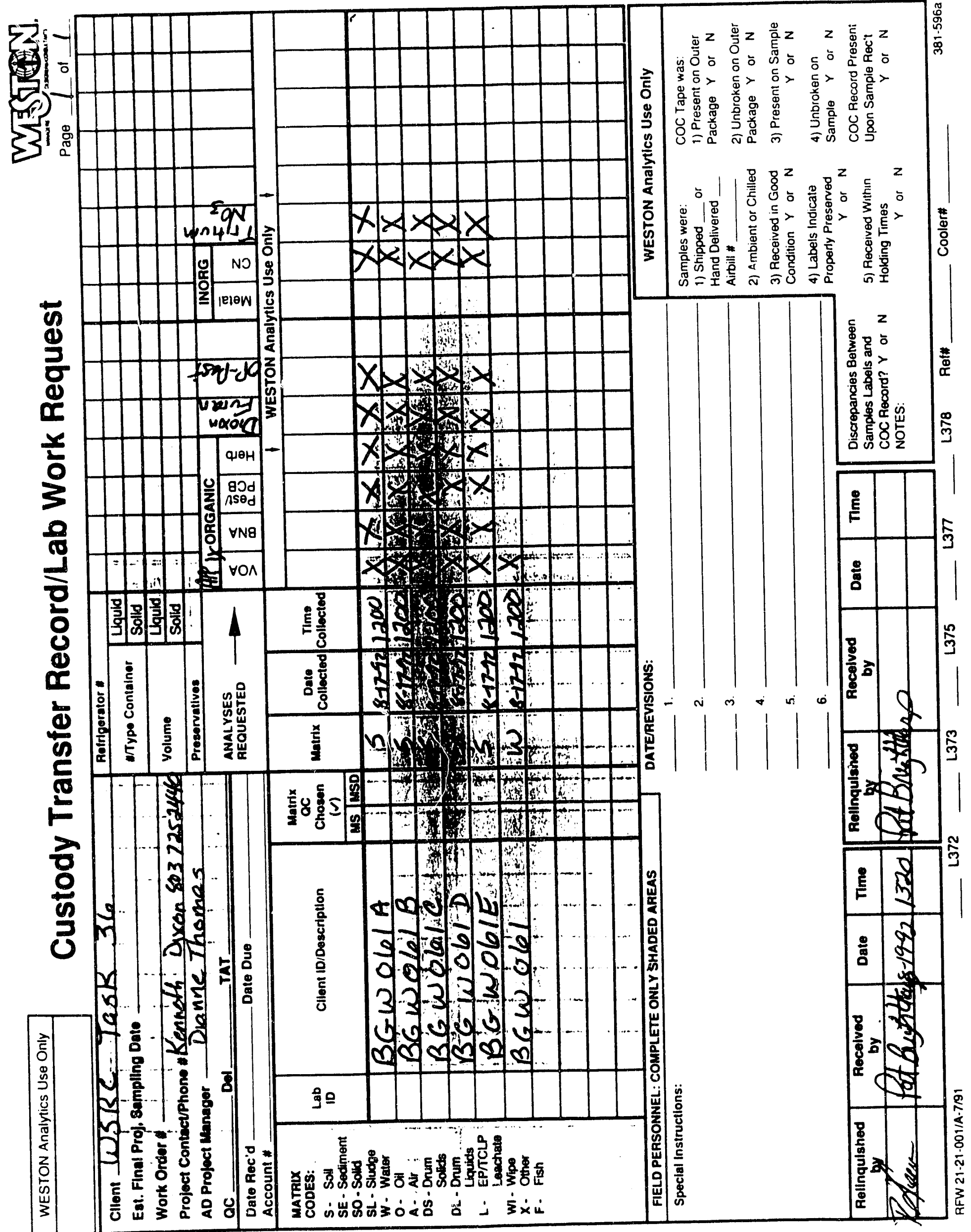




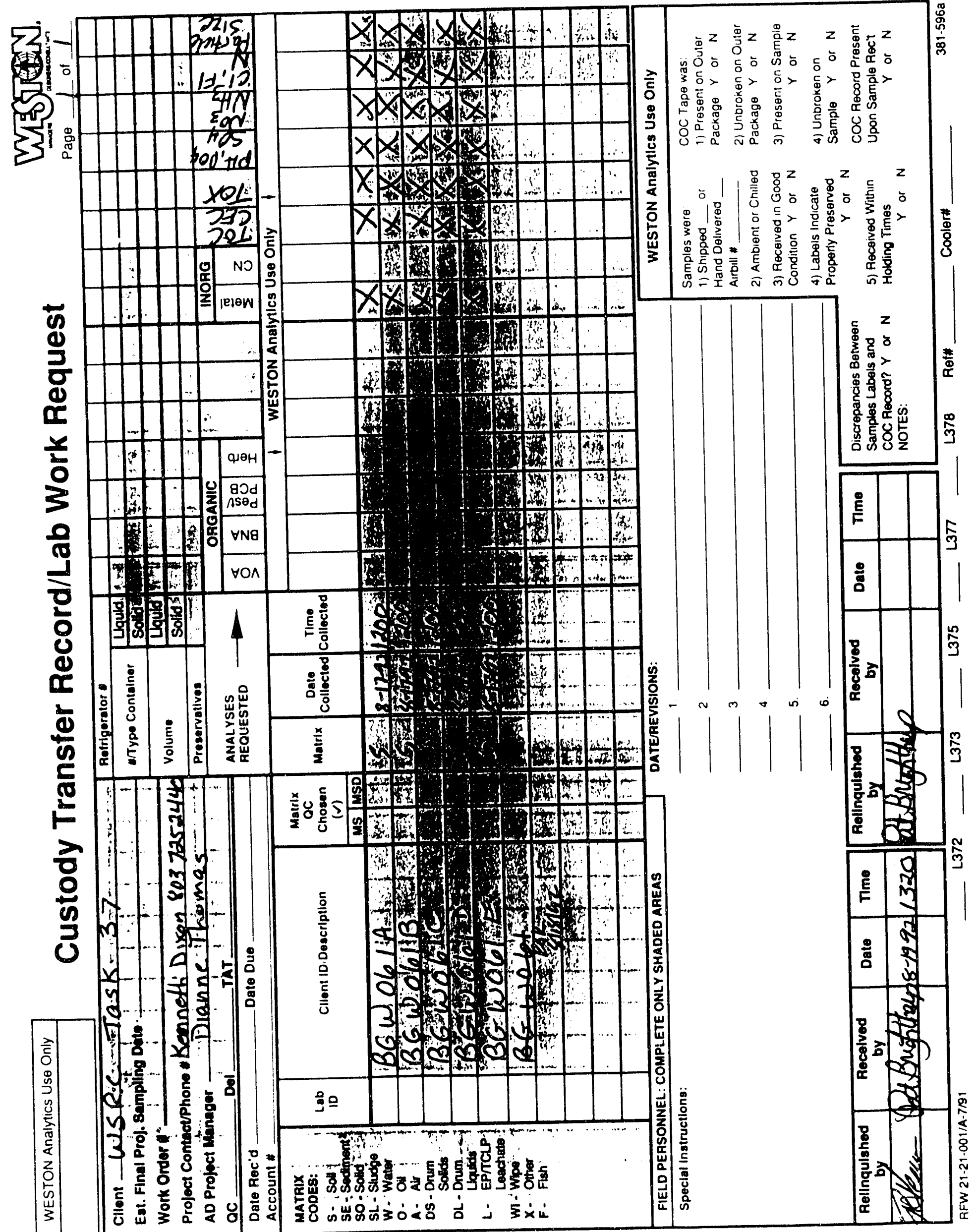




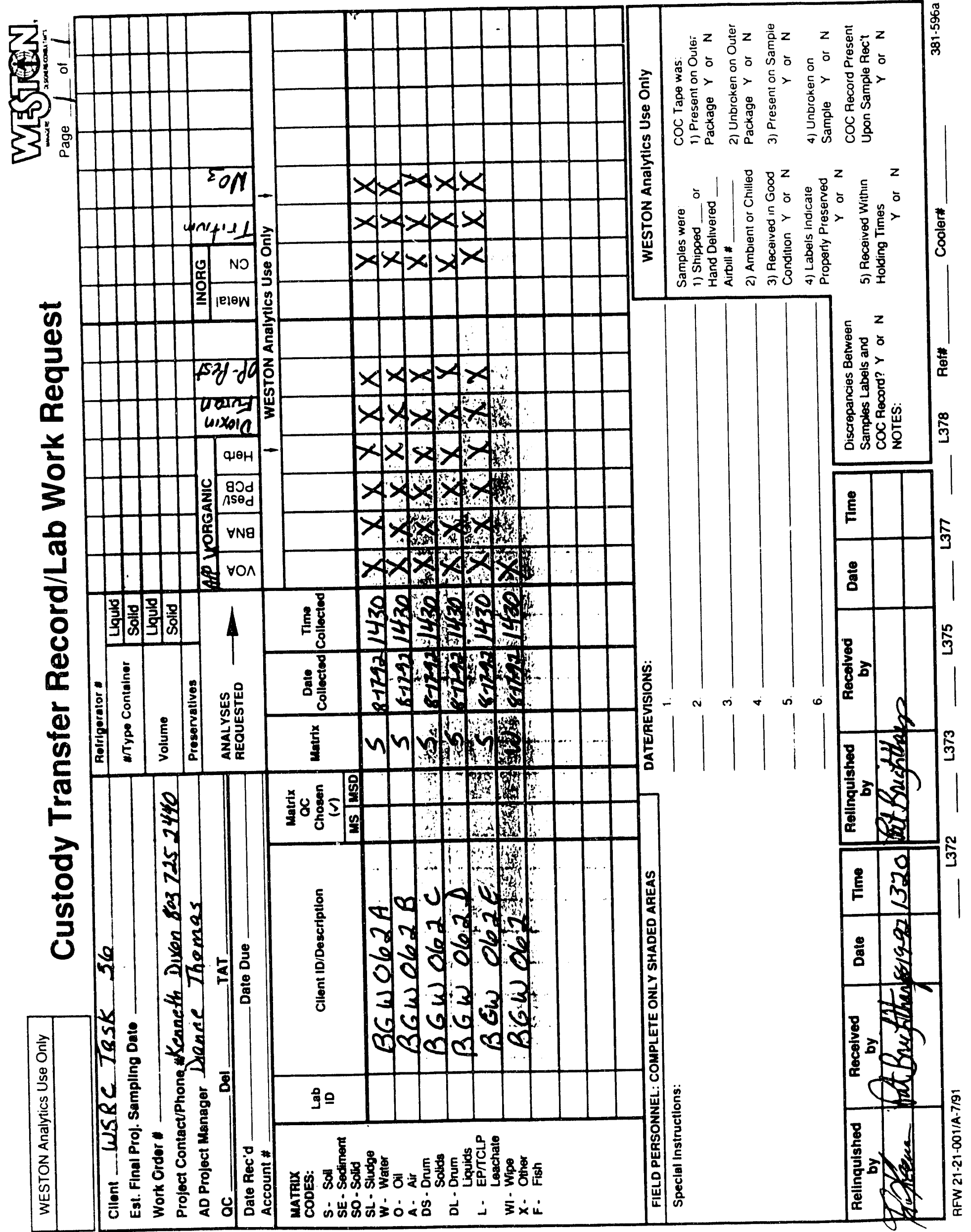




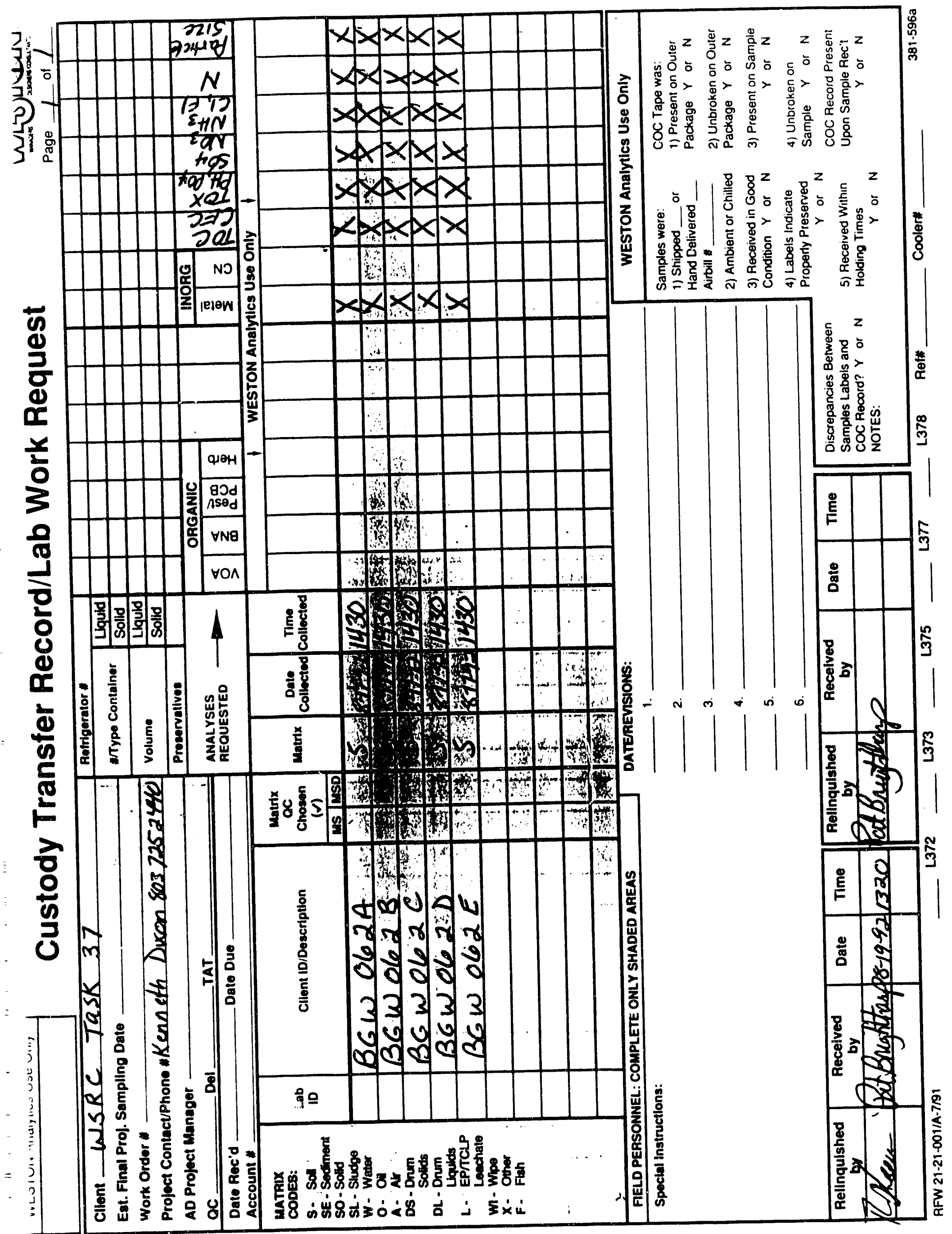




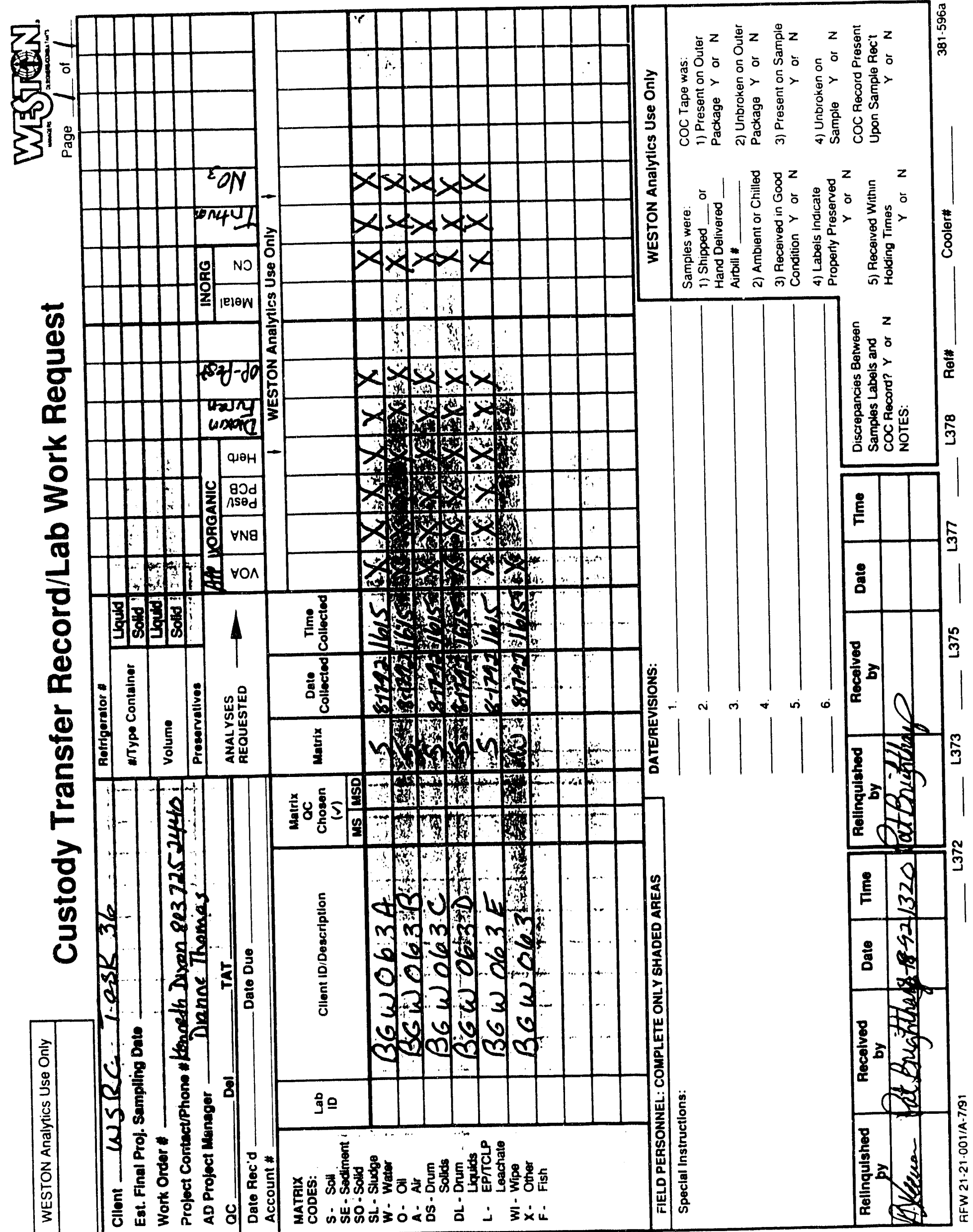




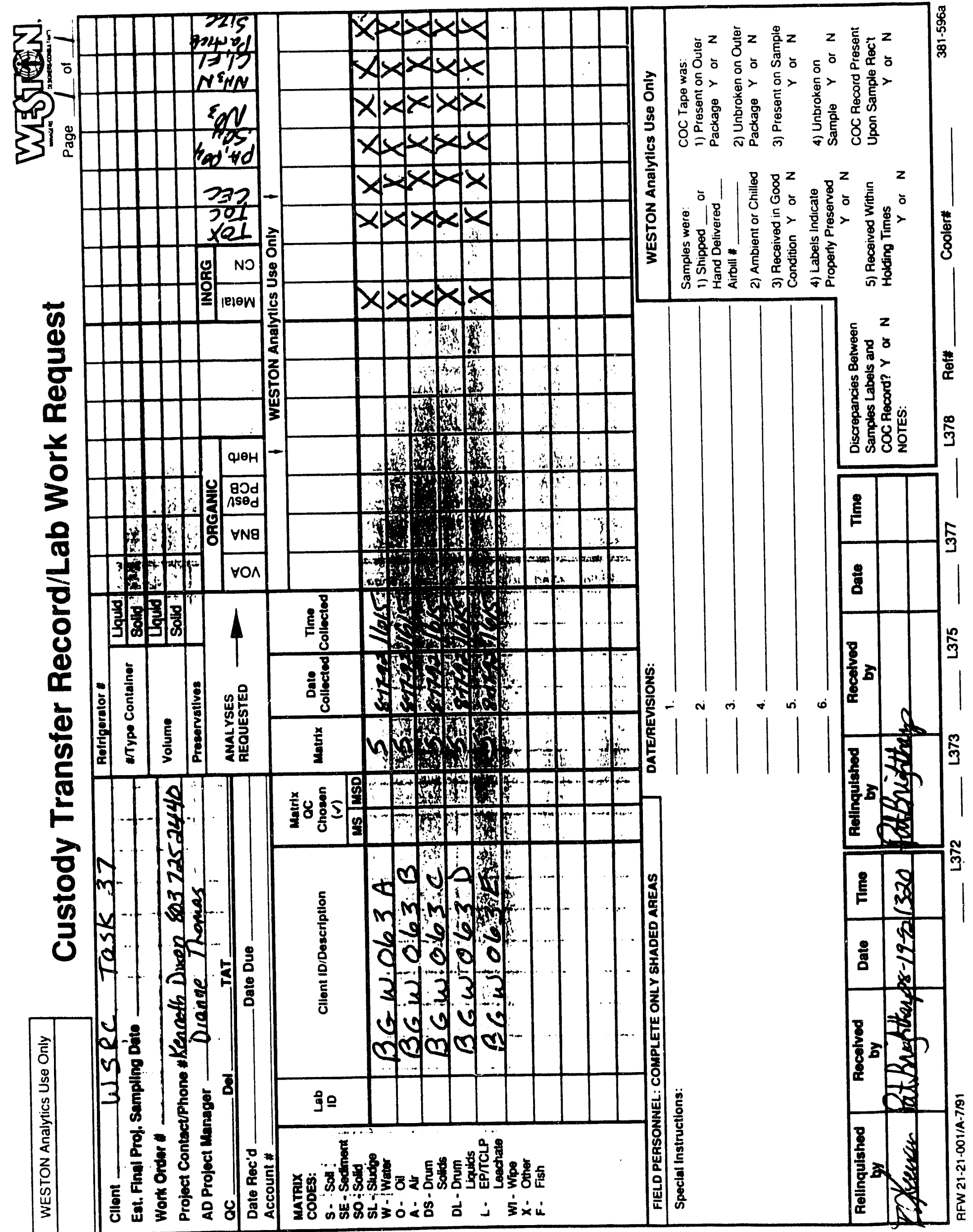




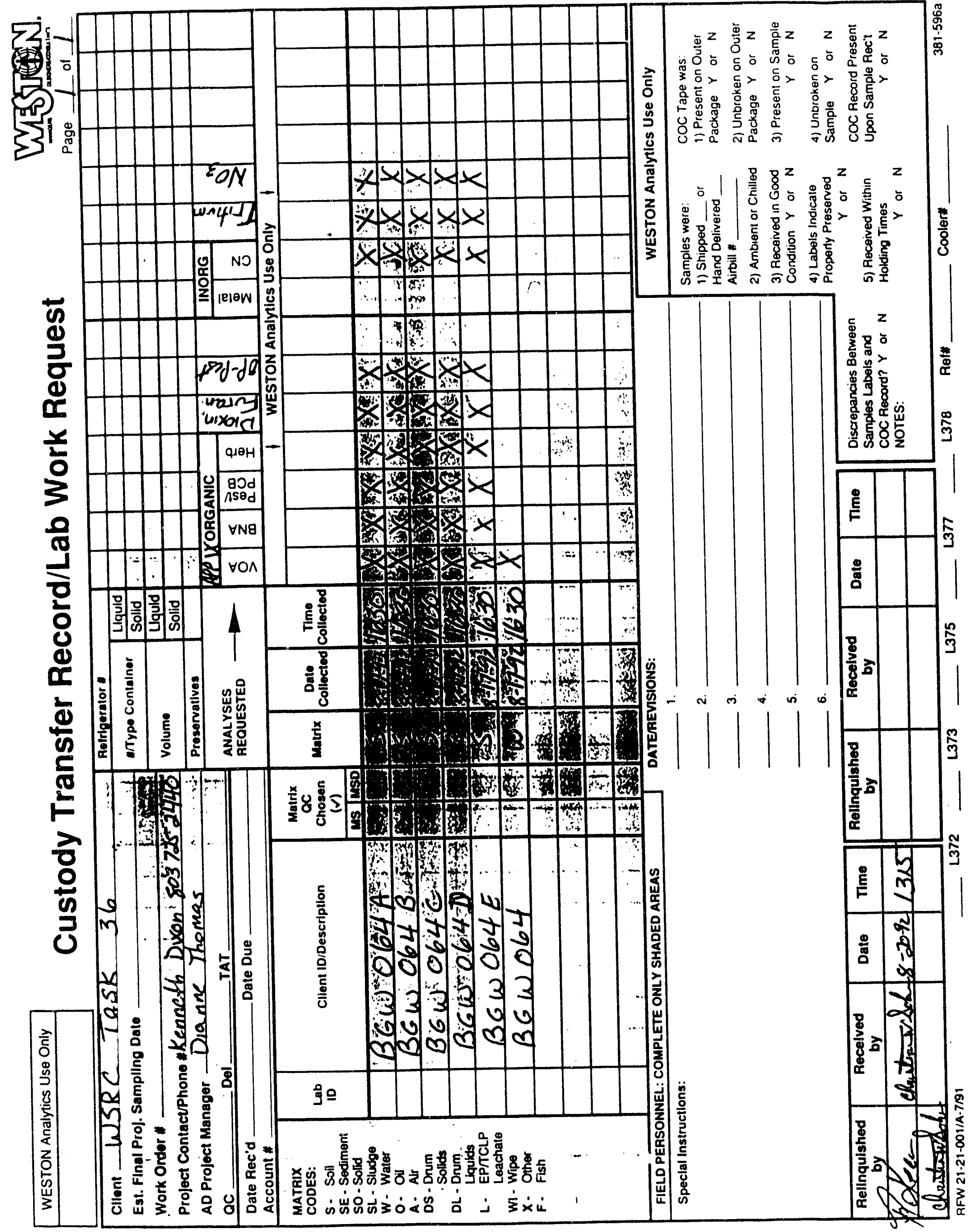




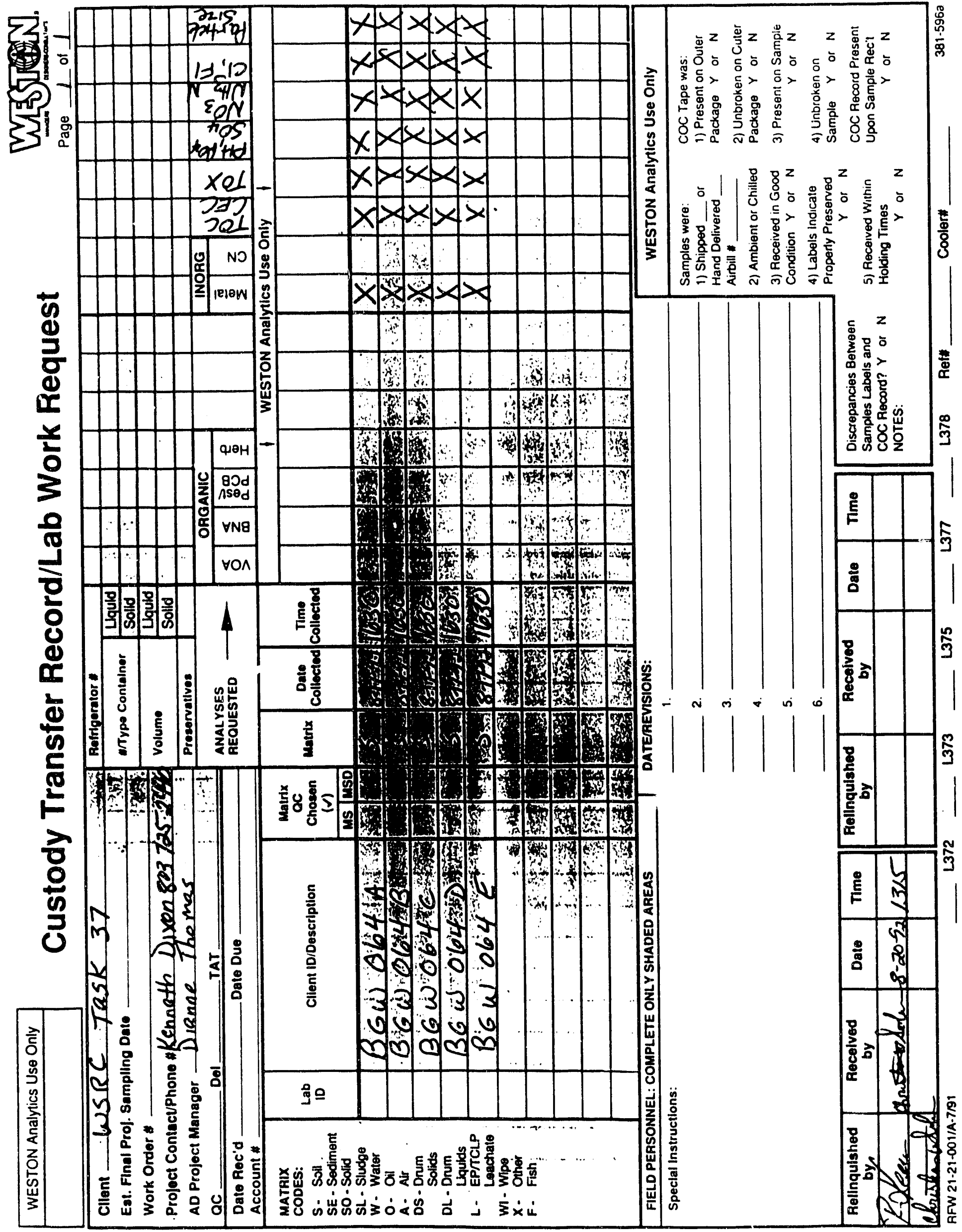




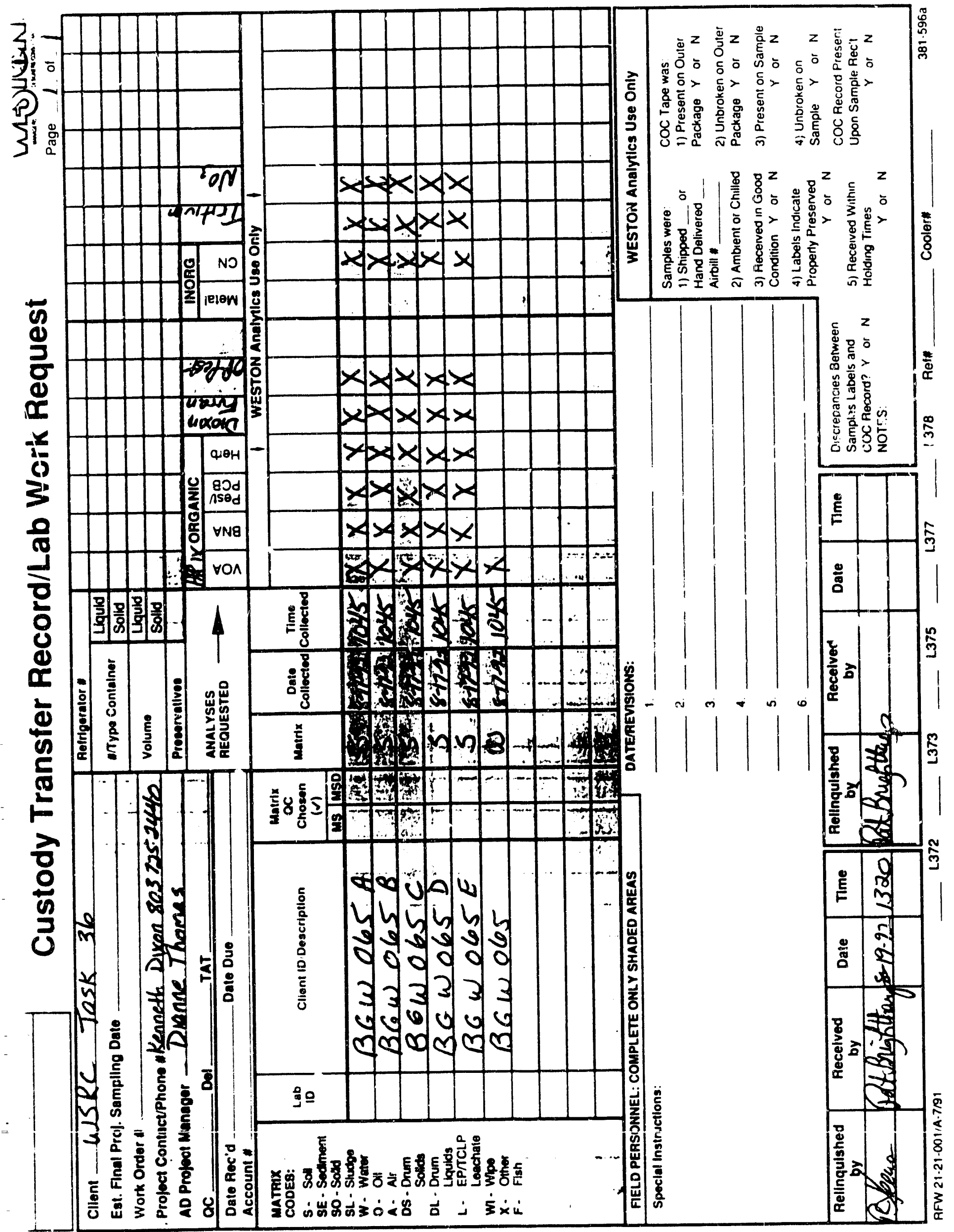




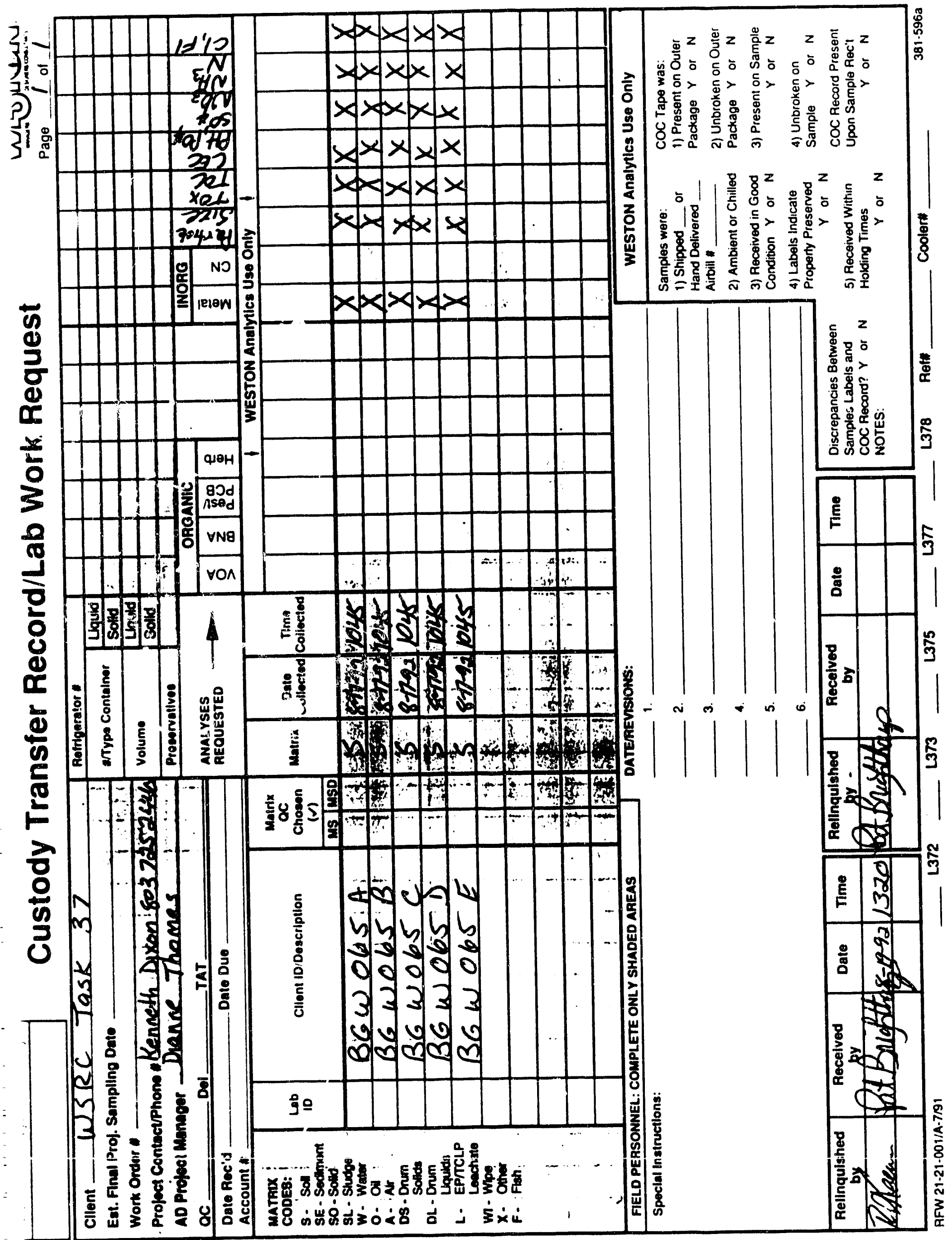




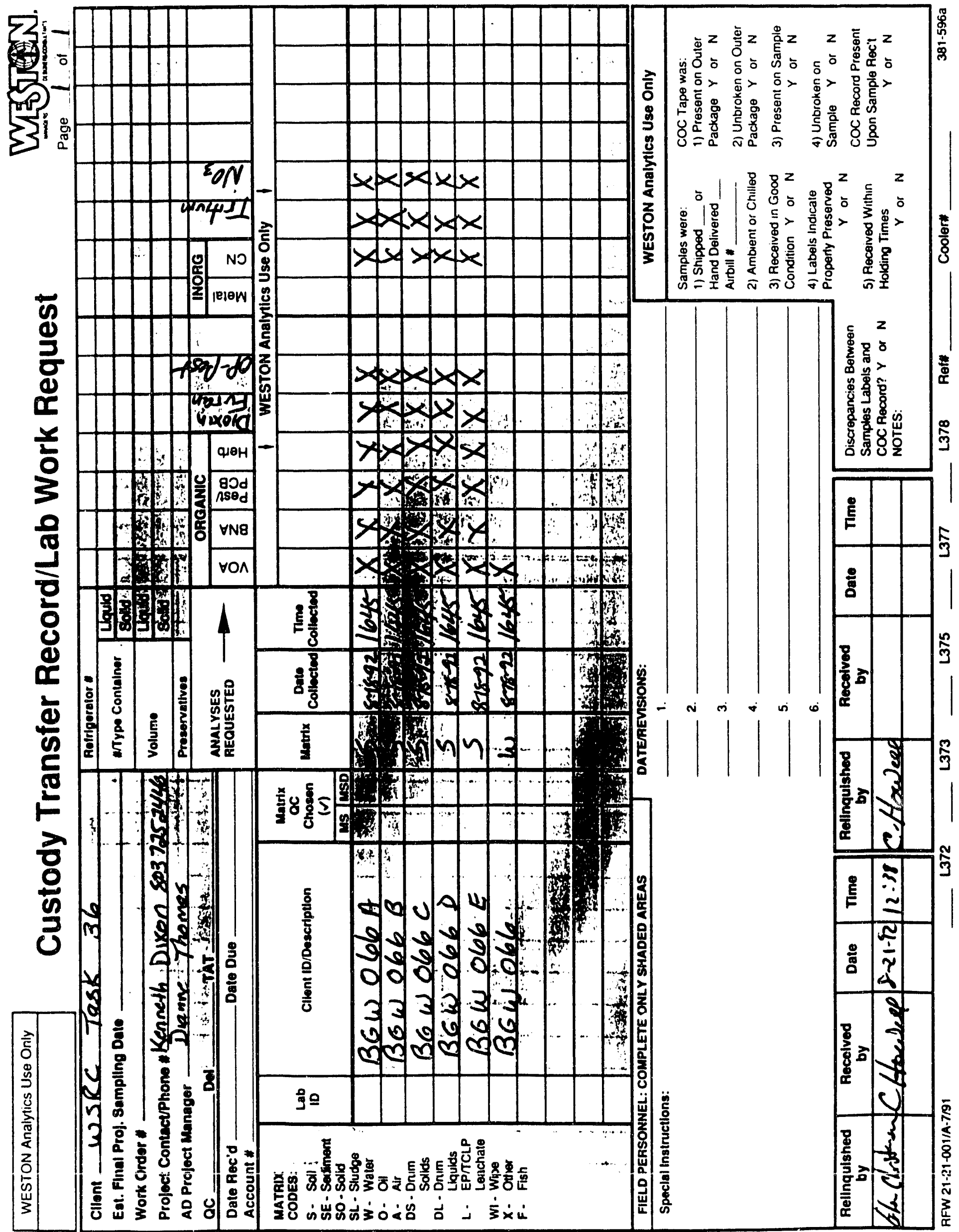




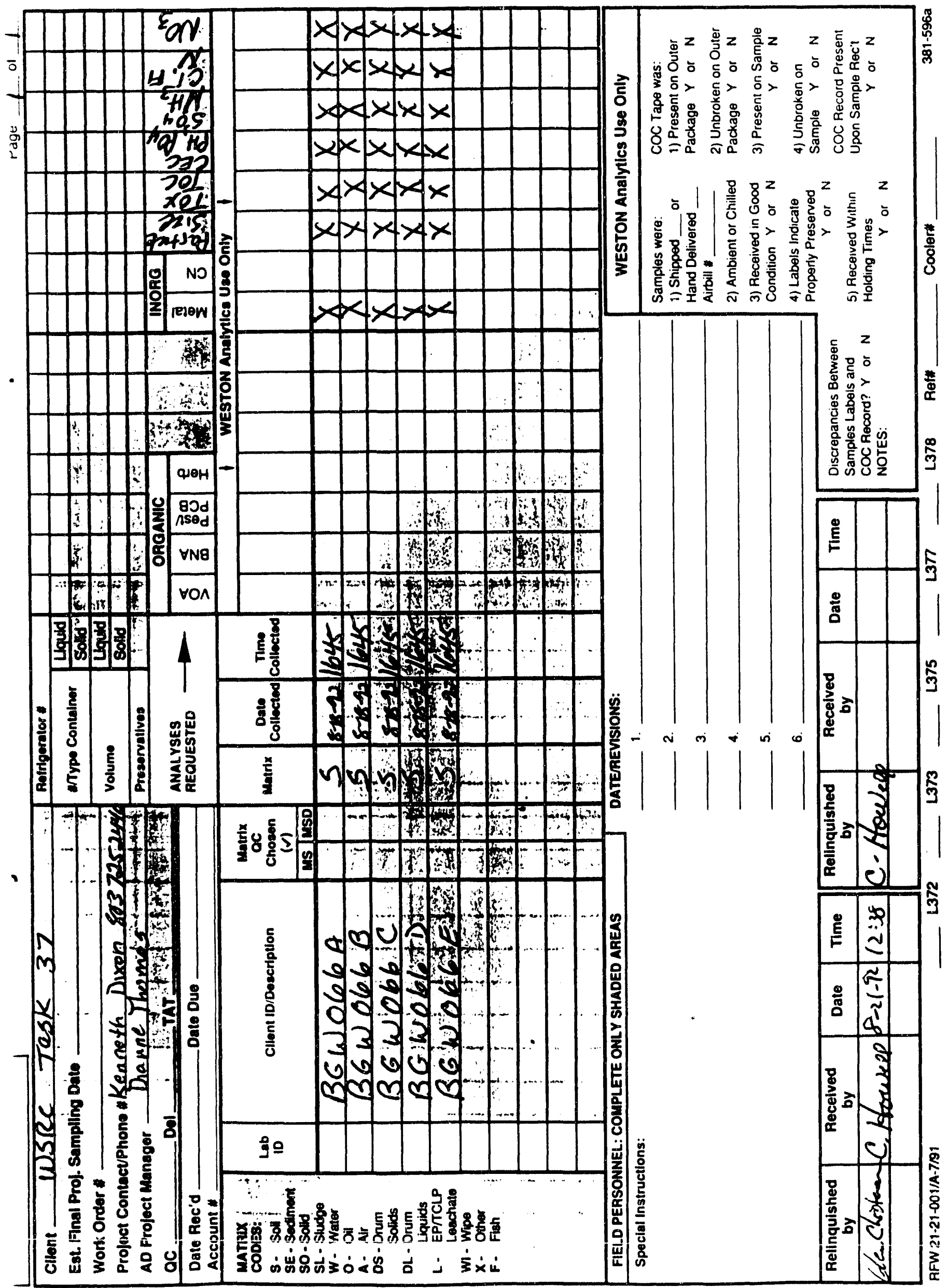




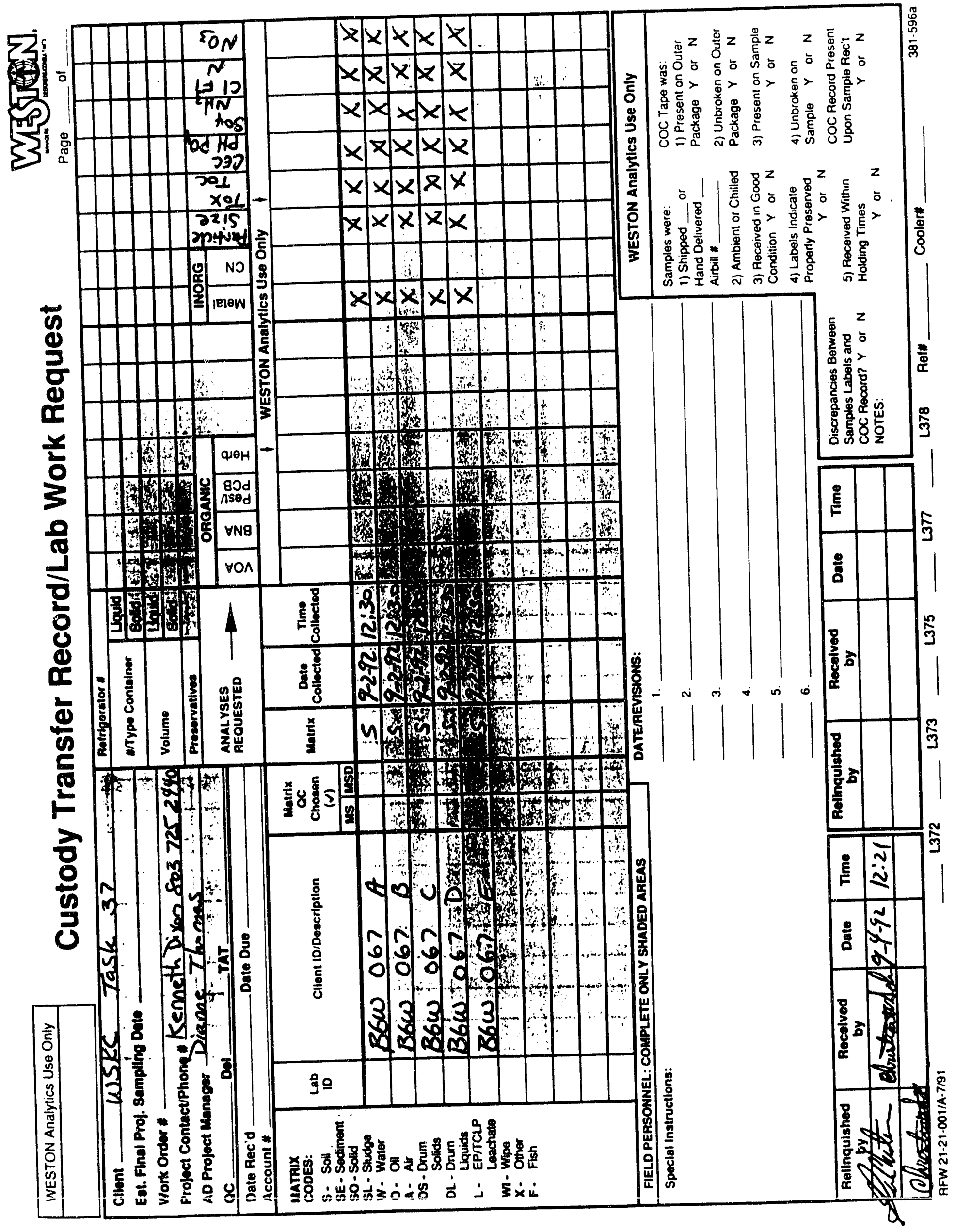




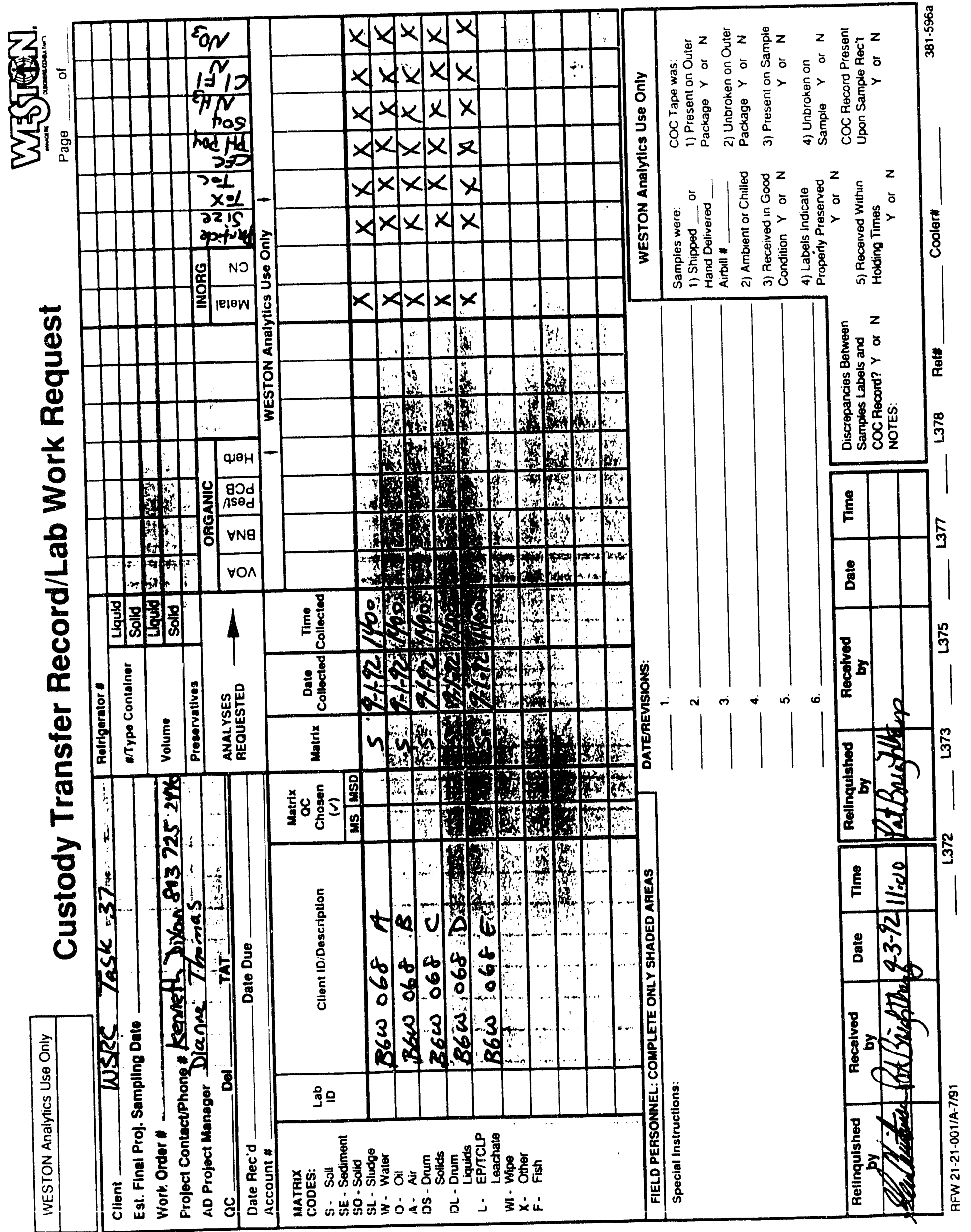




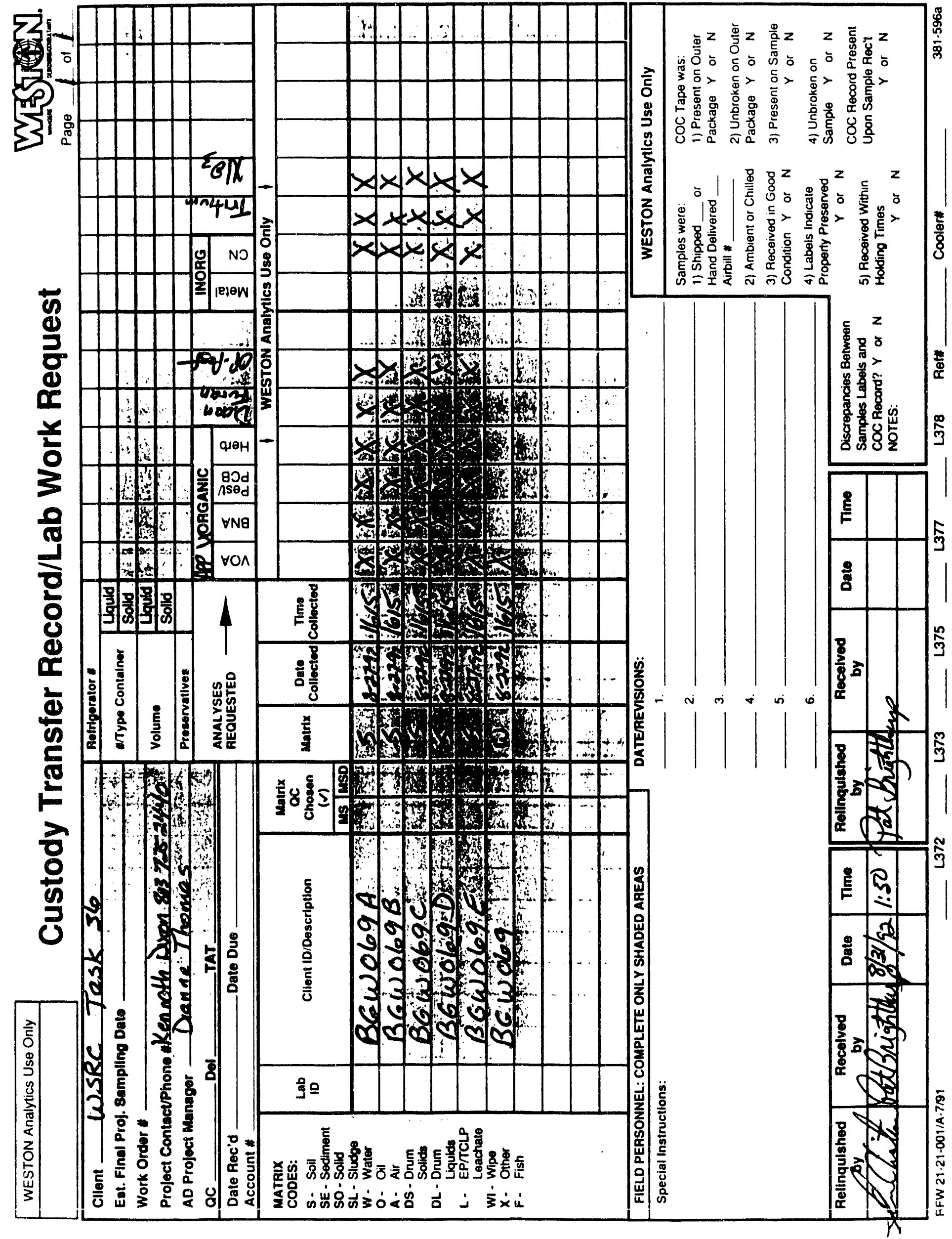




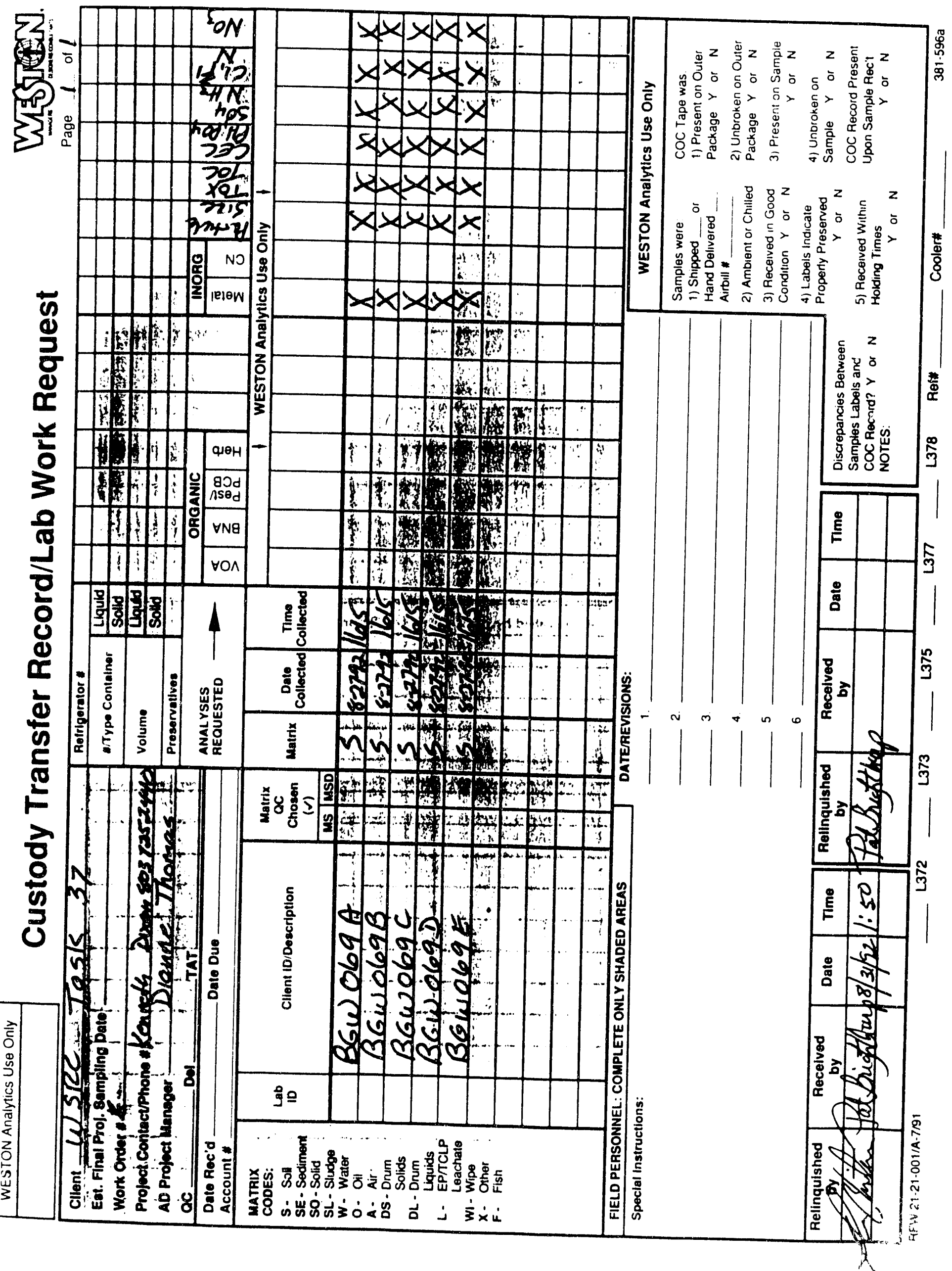




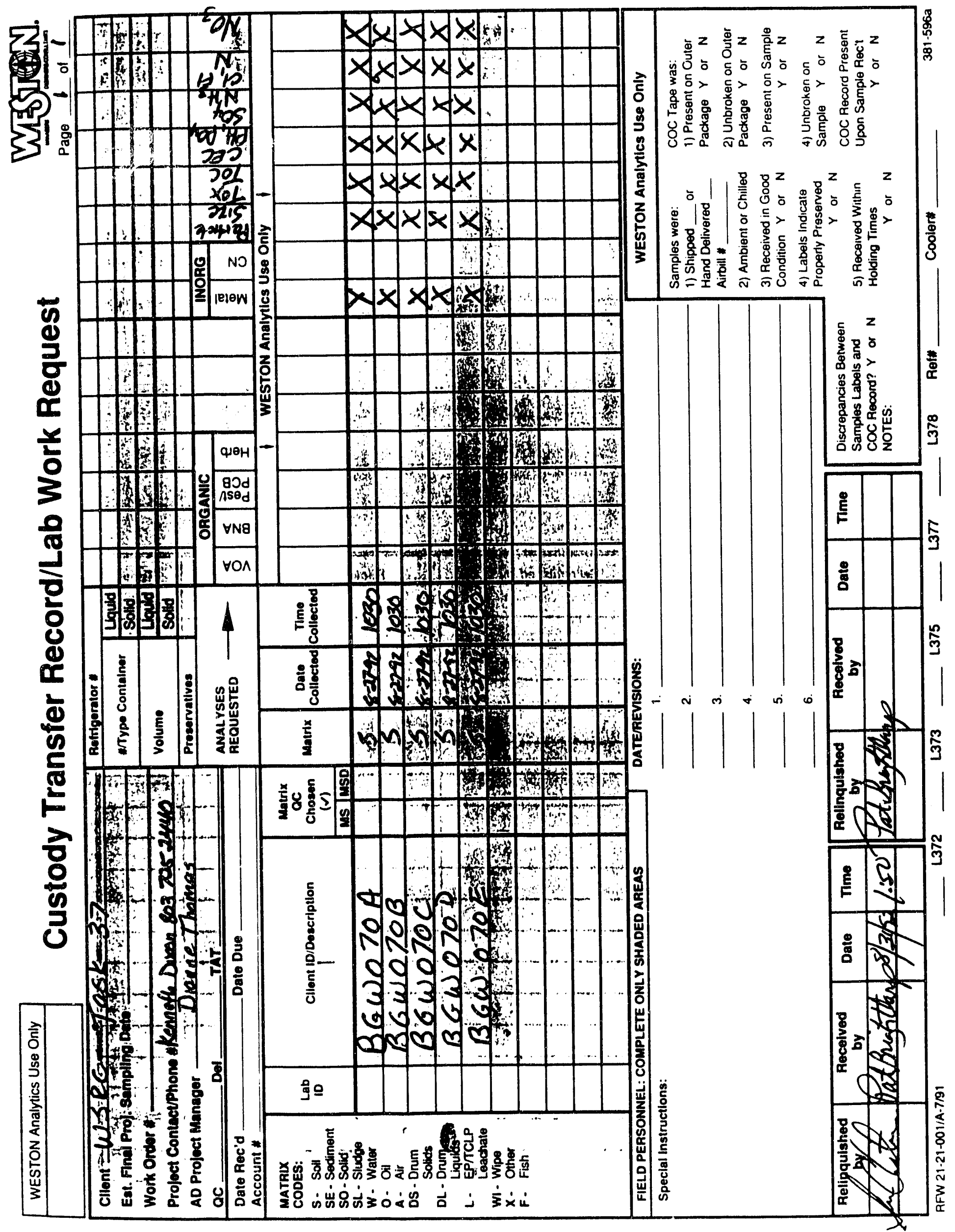




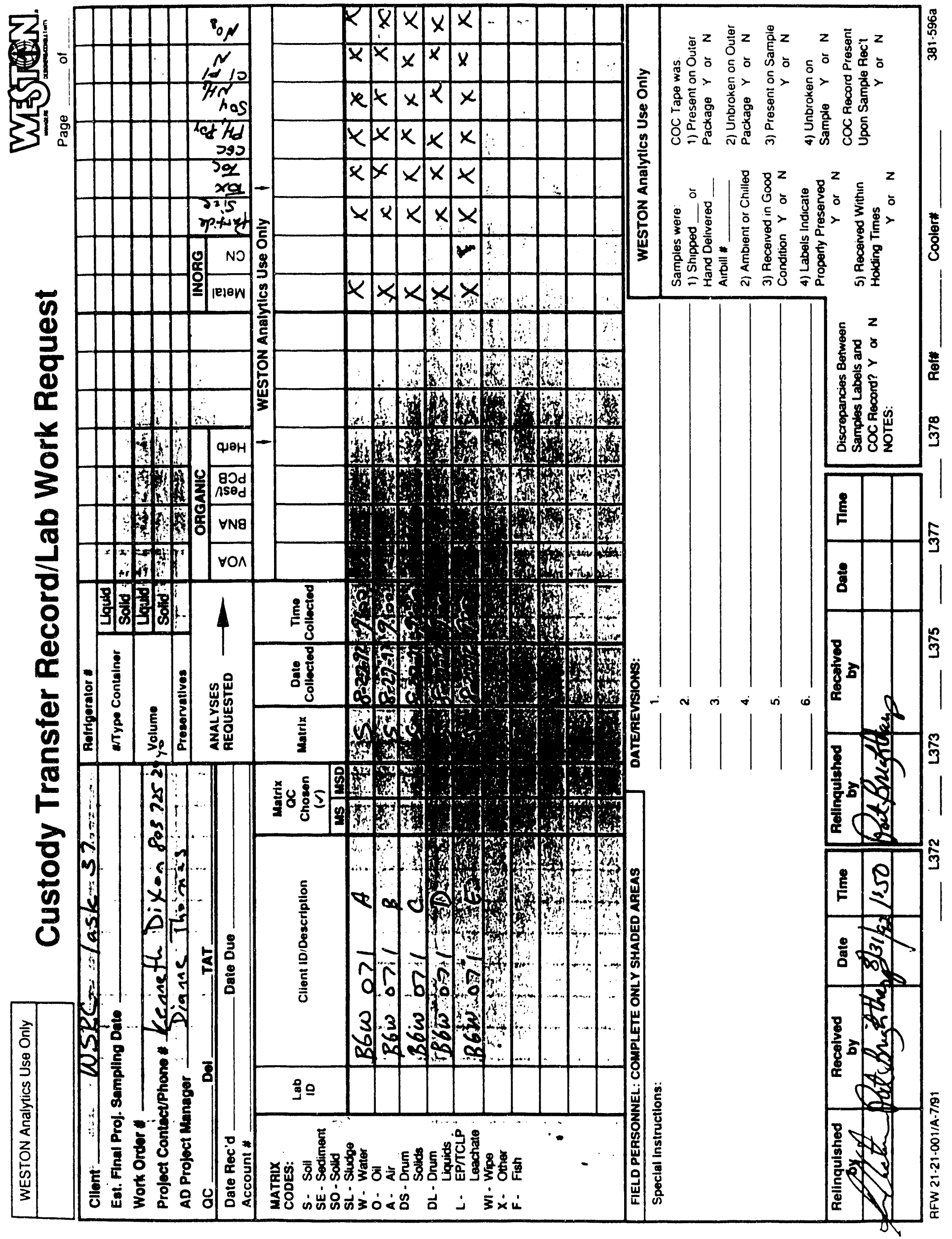




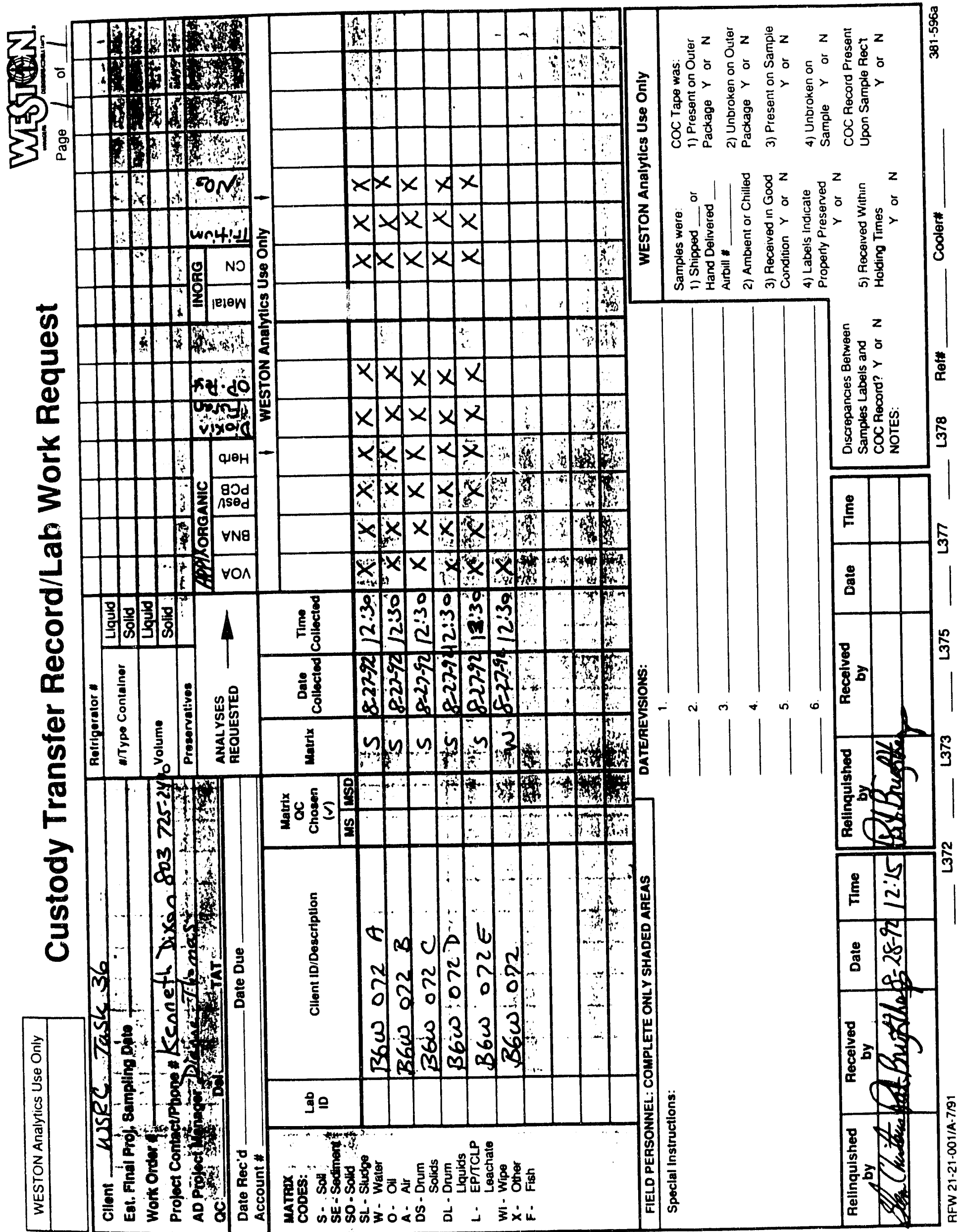




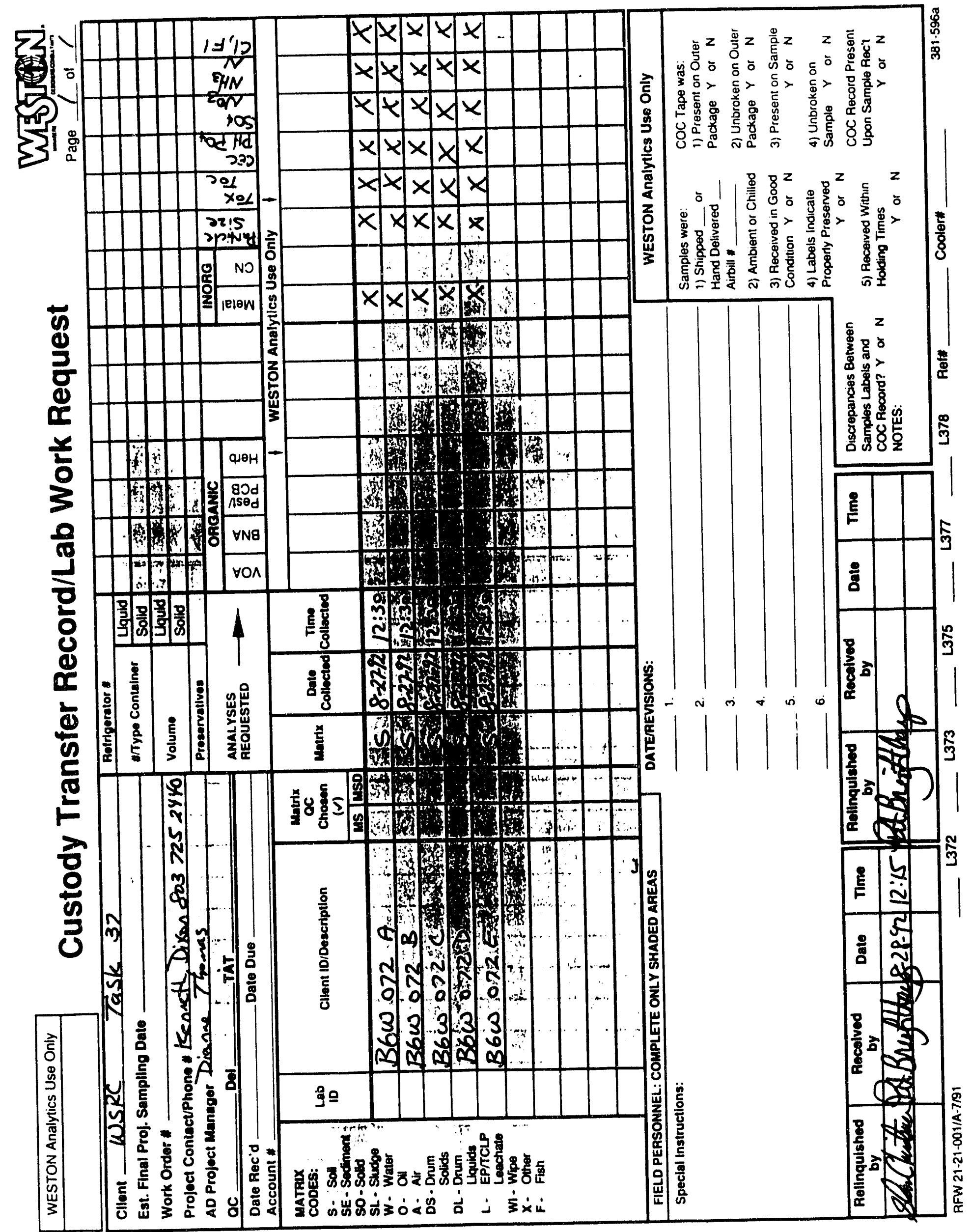




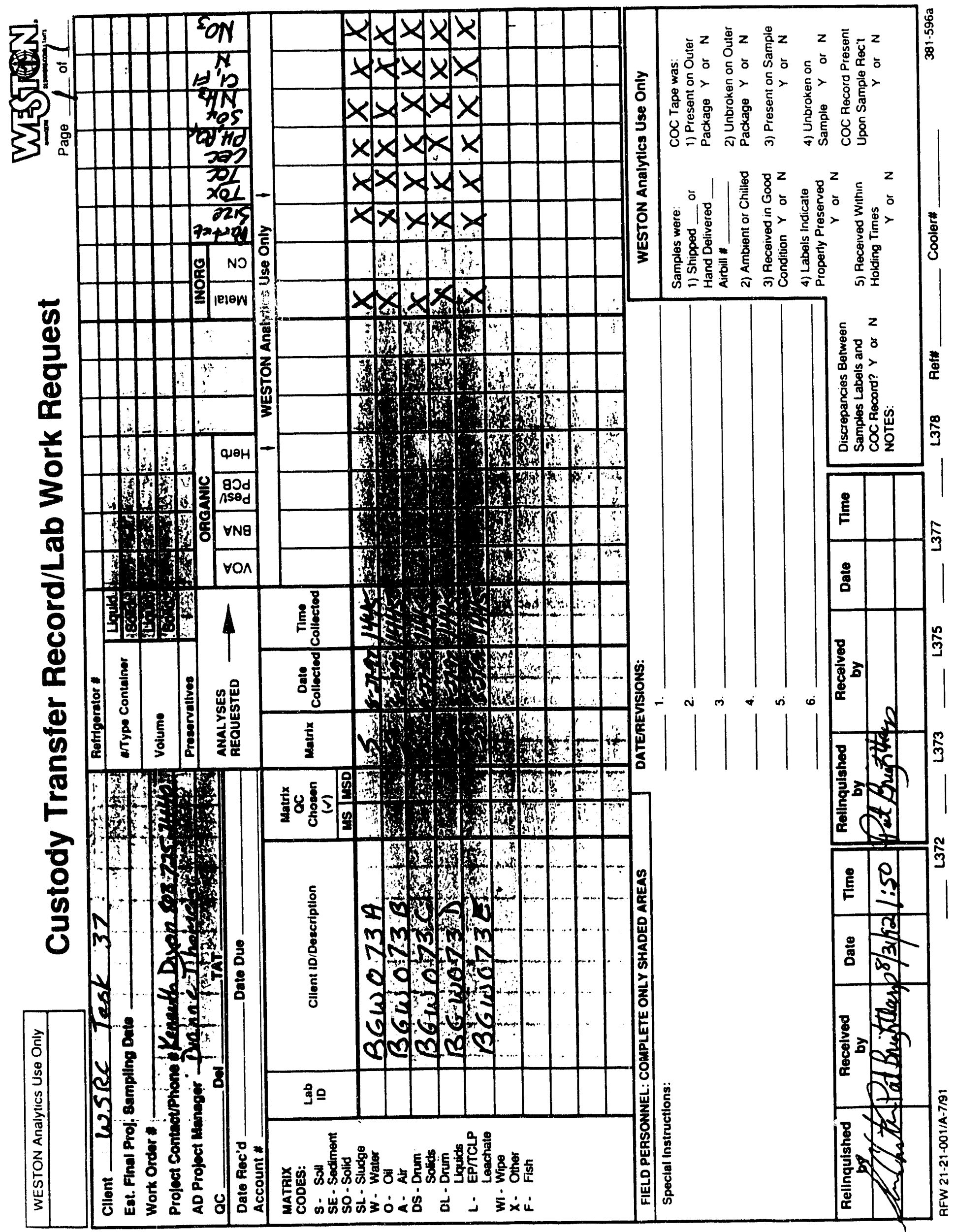




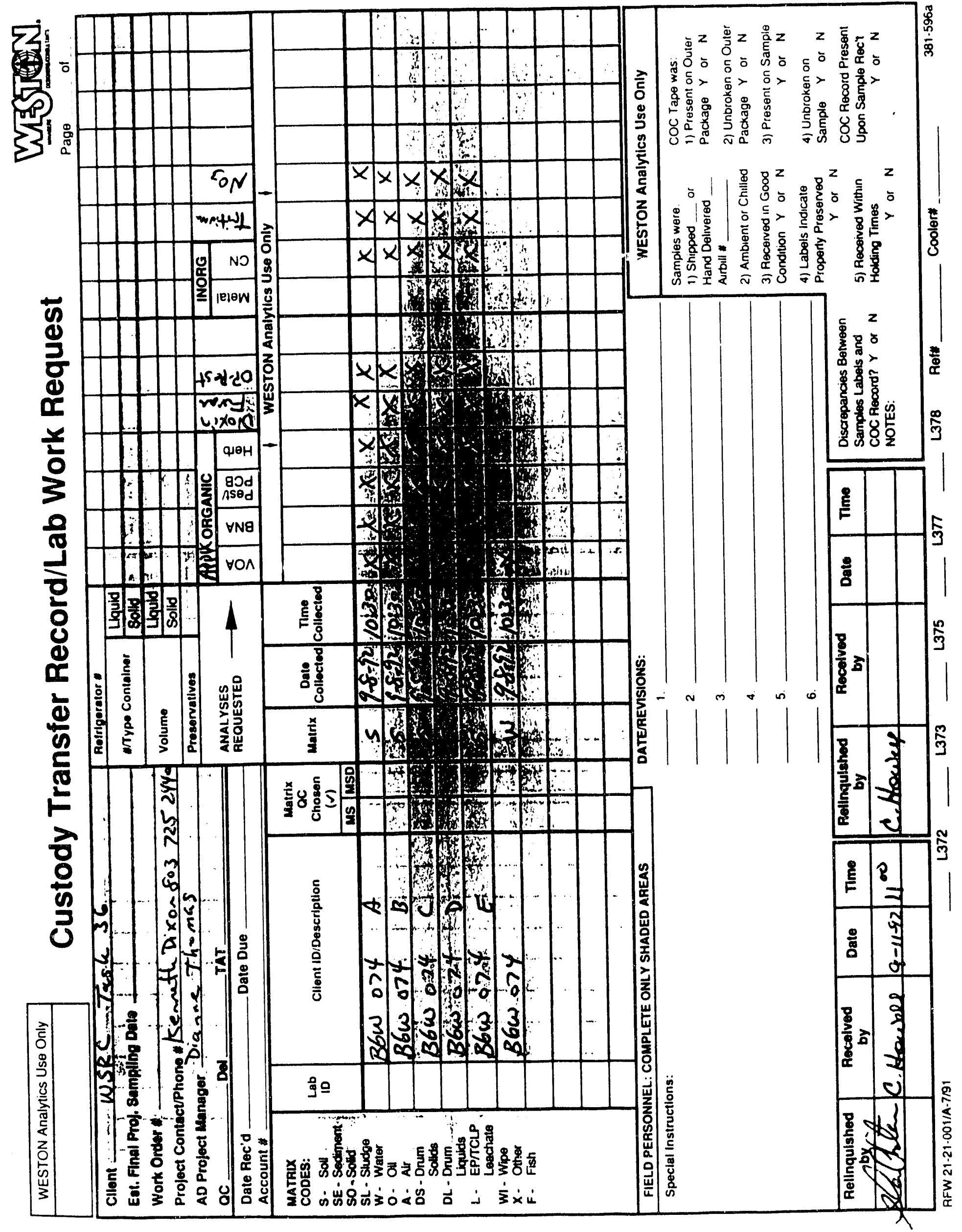




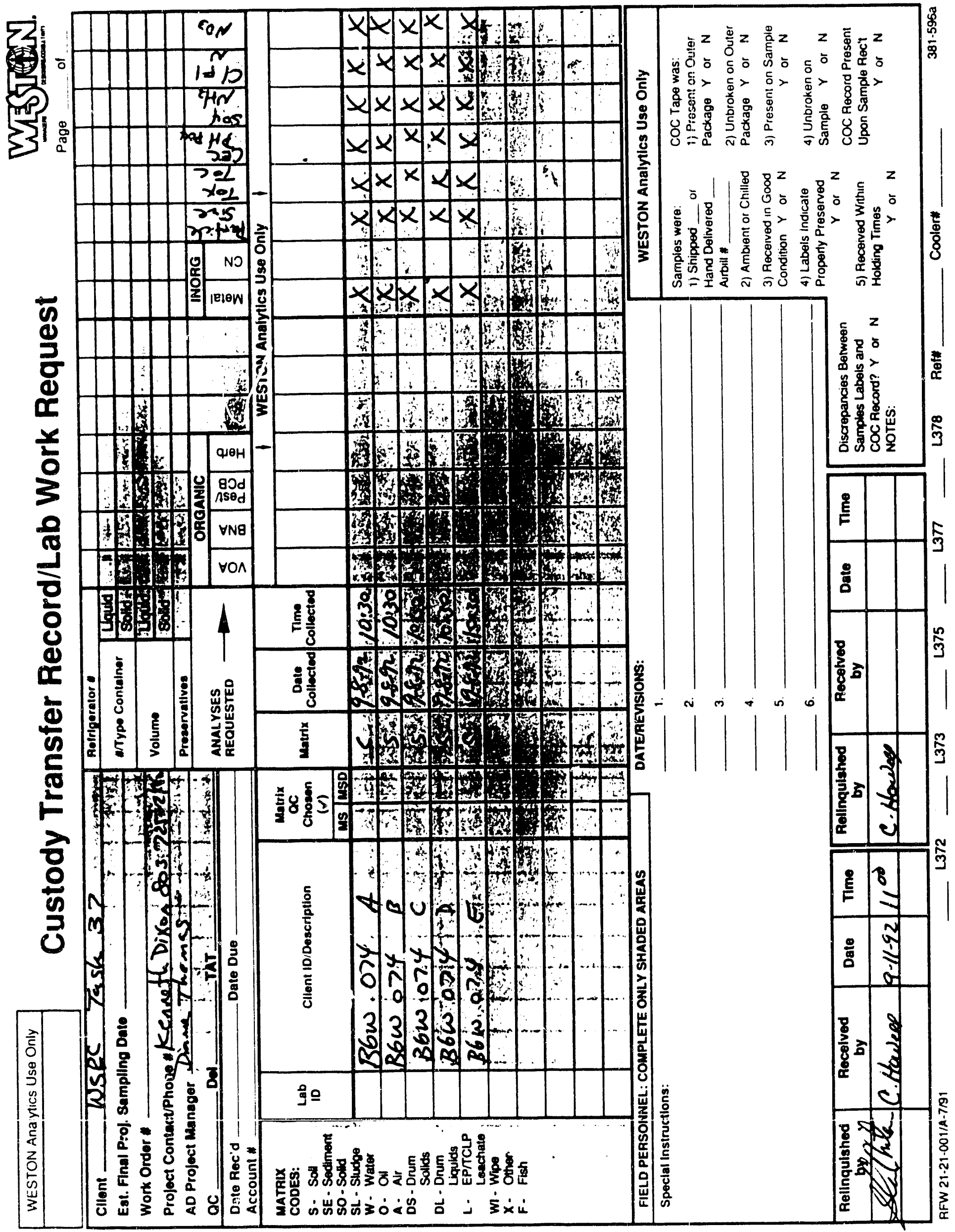


急
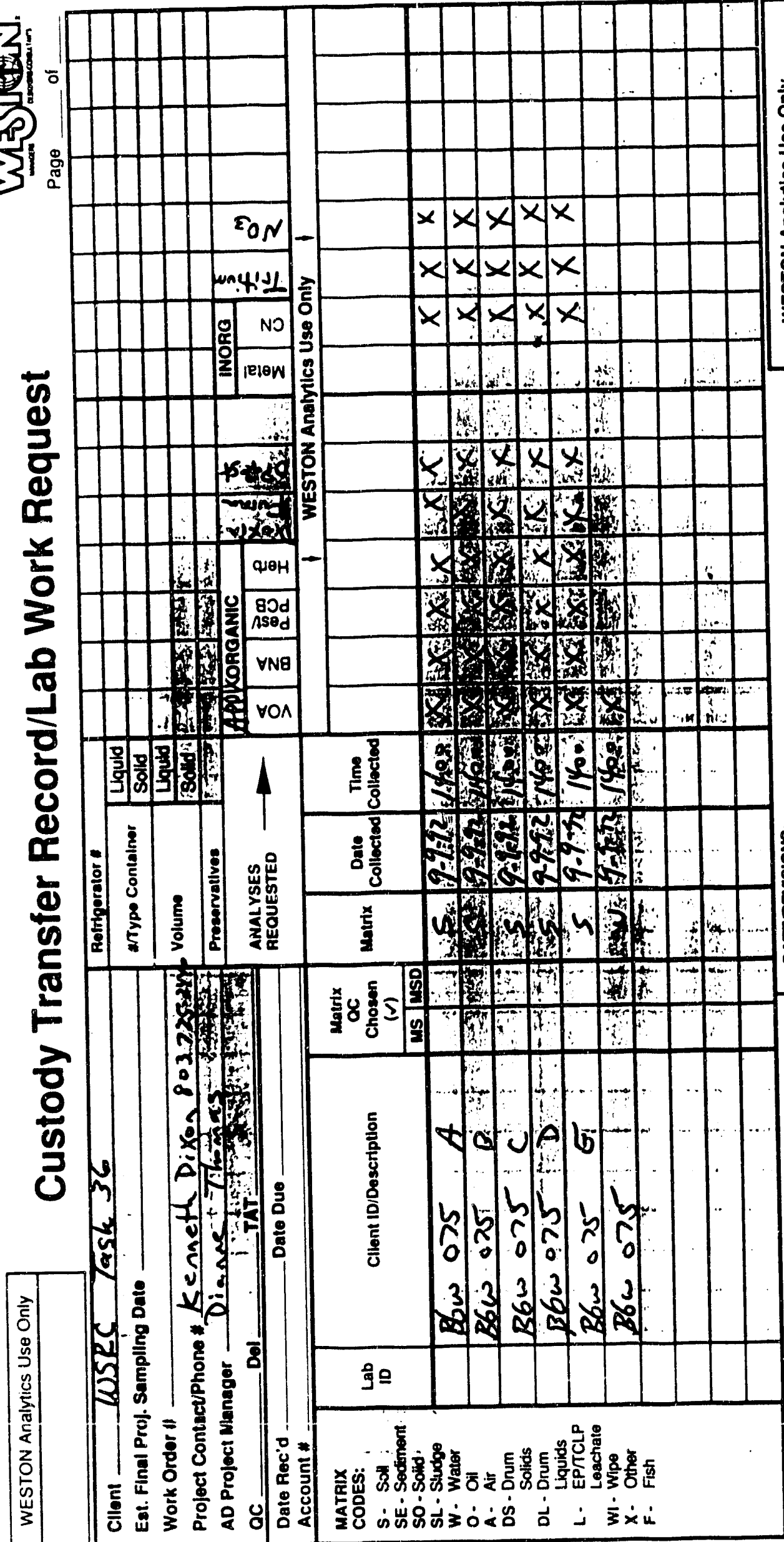
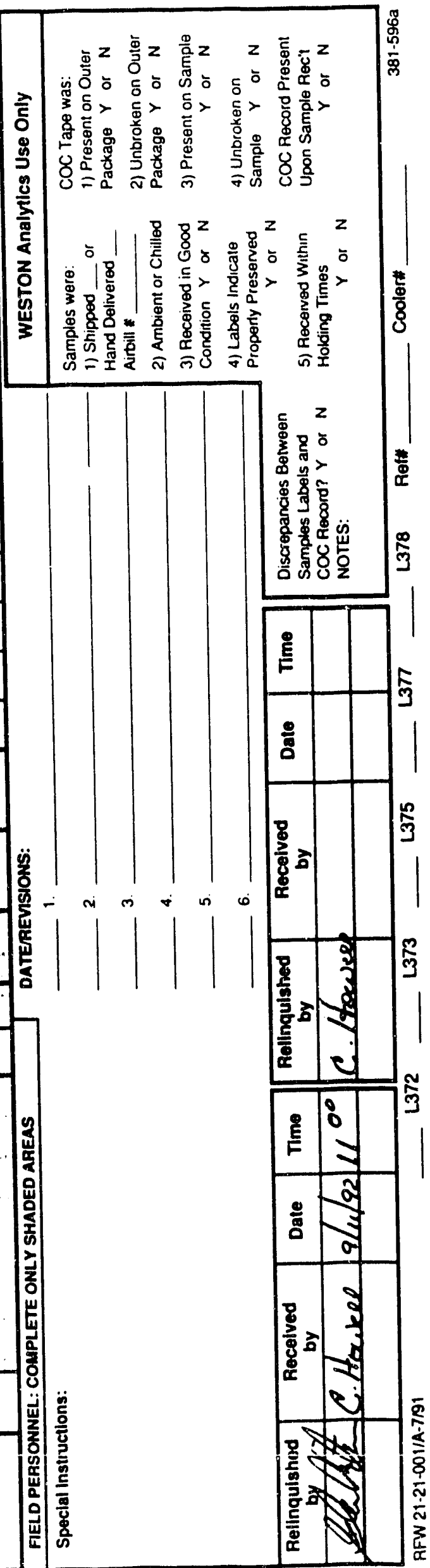


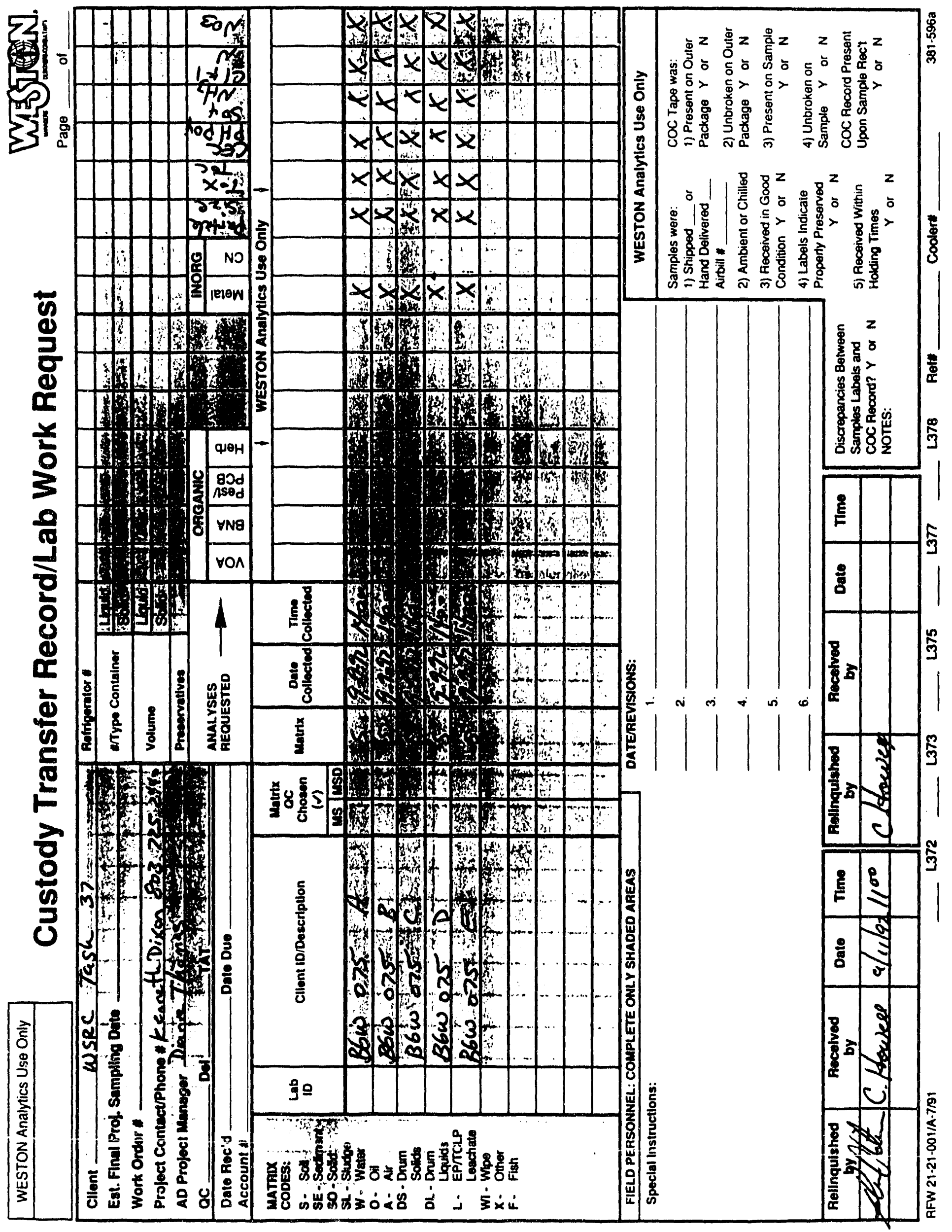




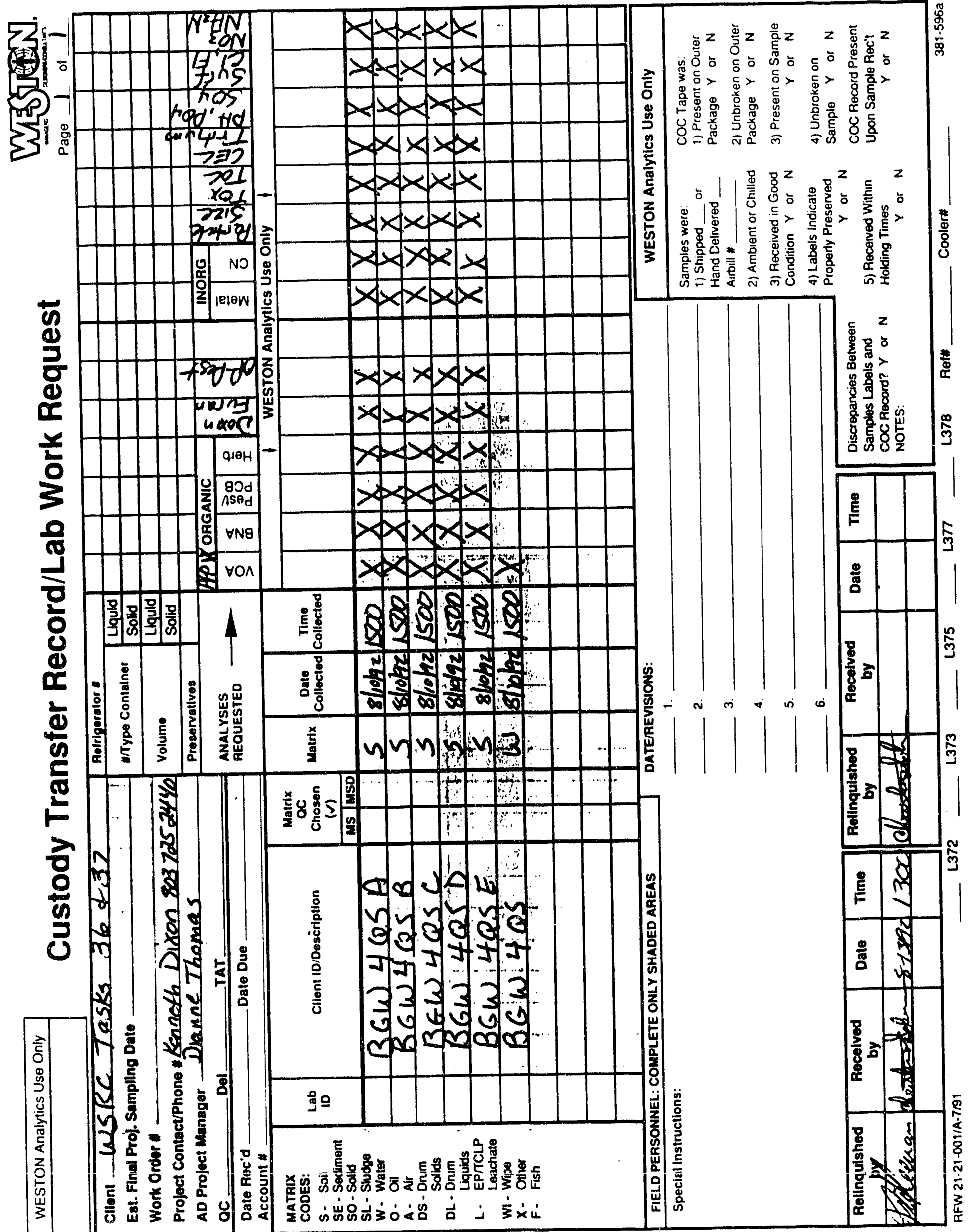




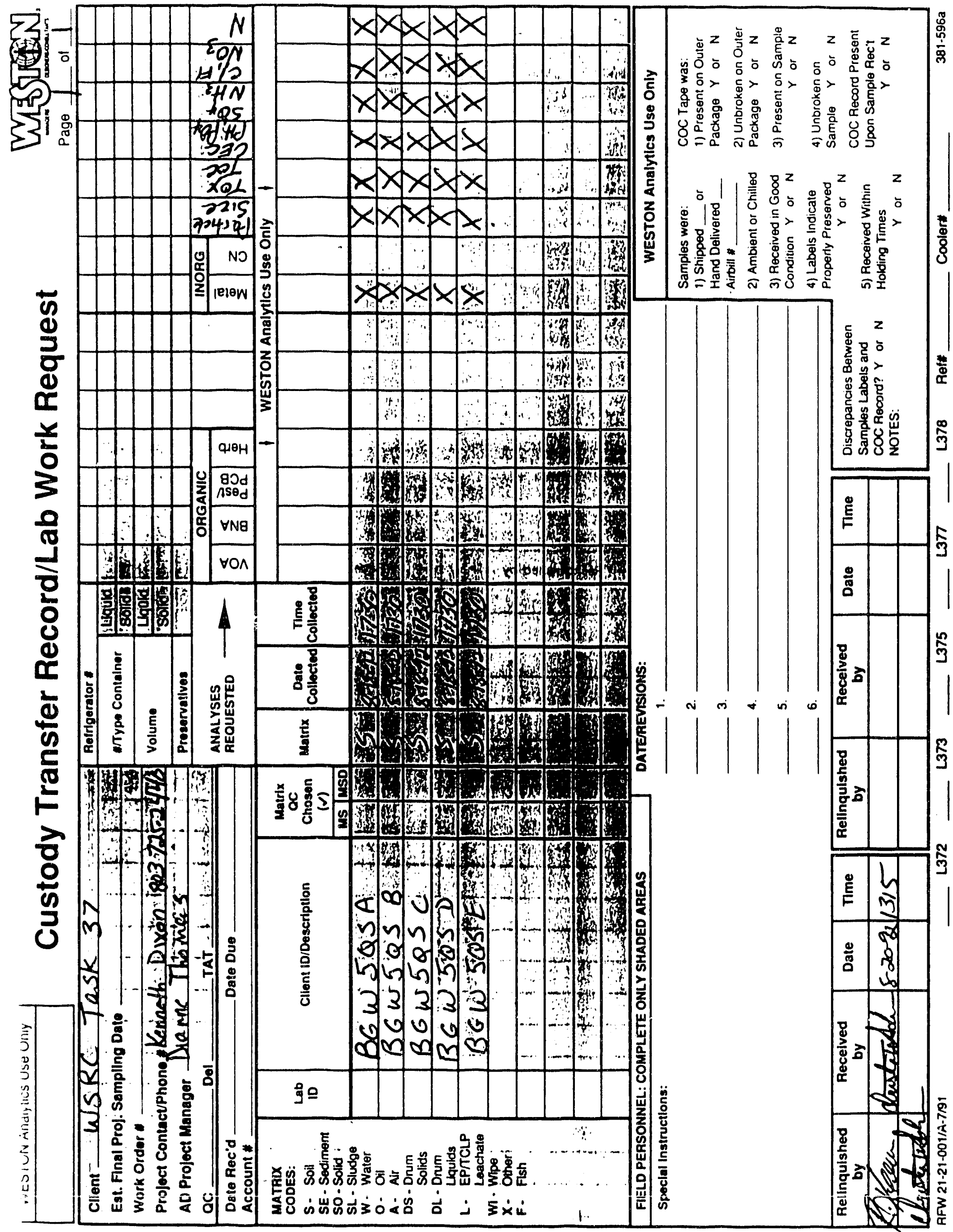




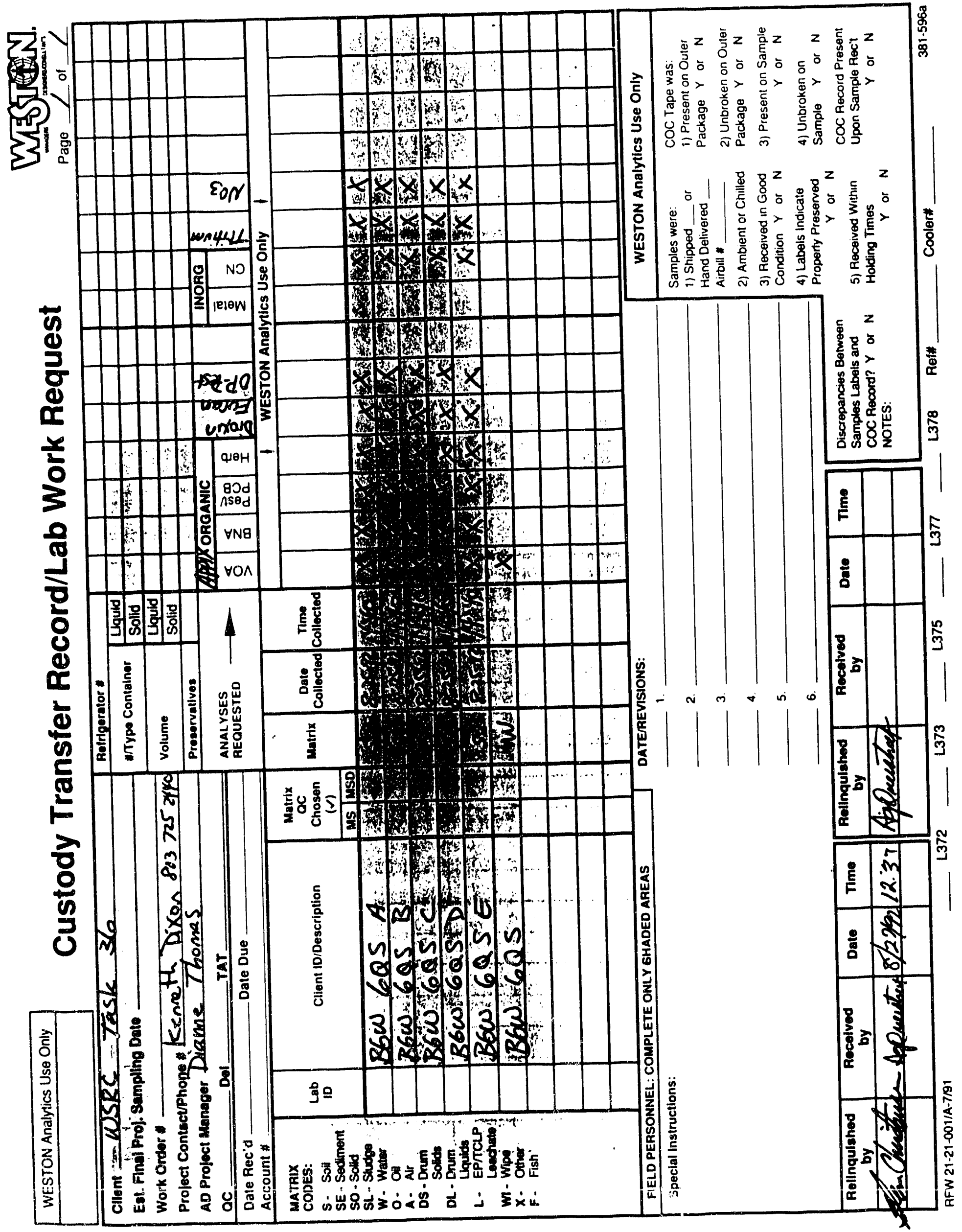




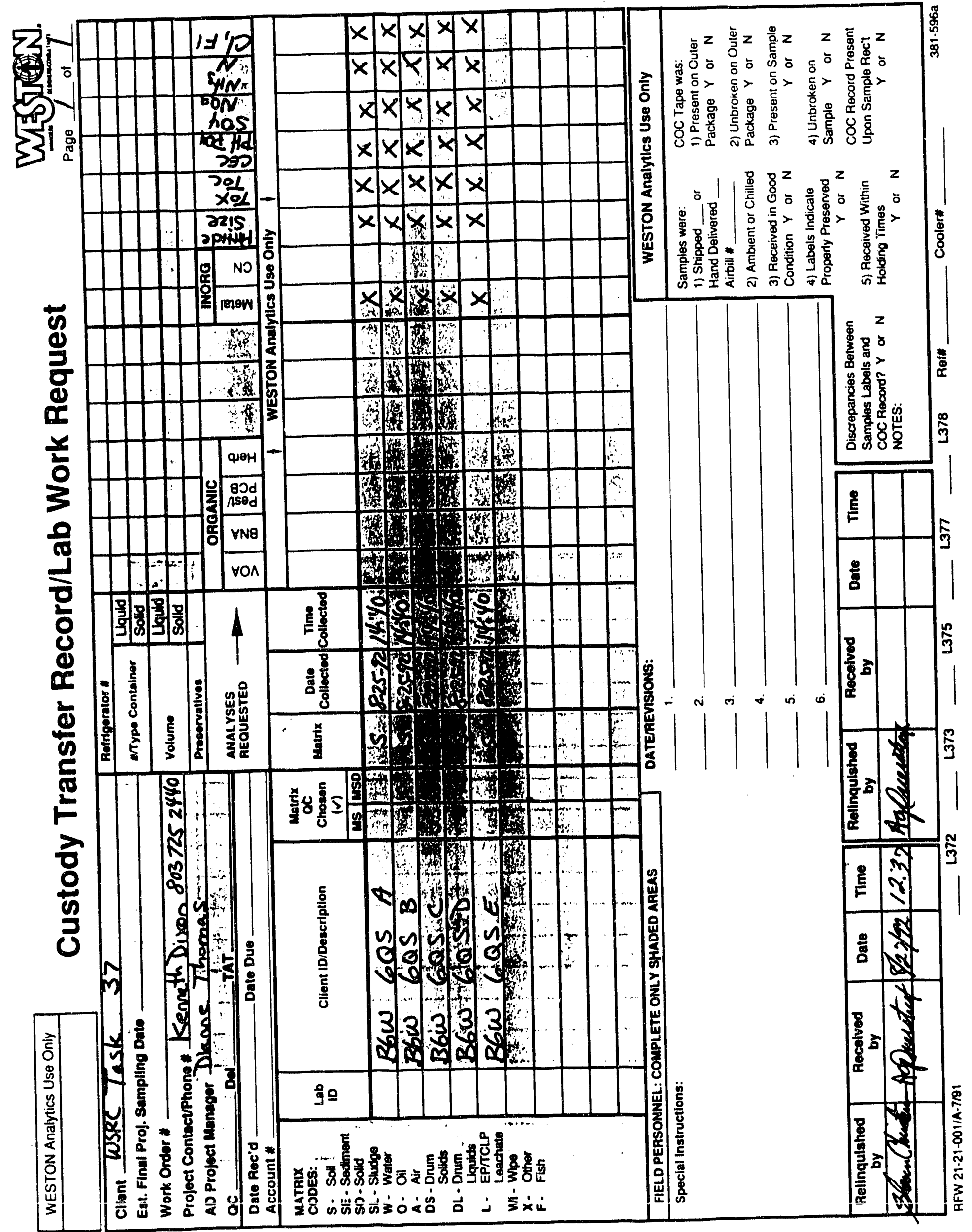




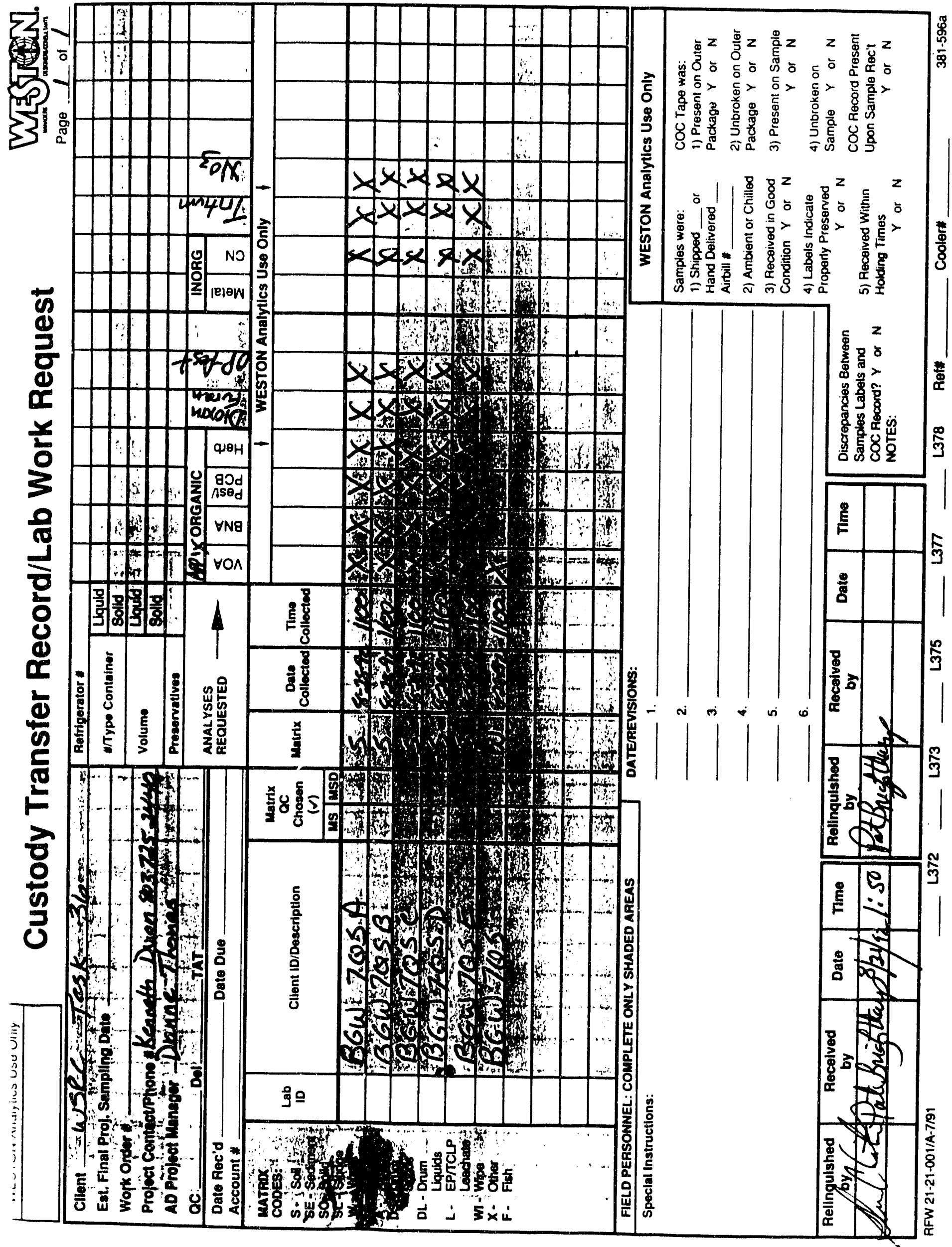




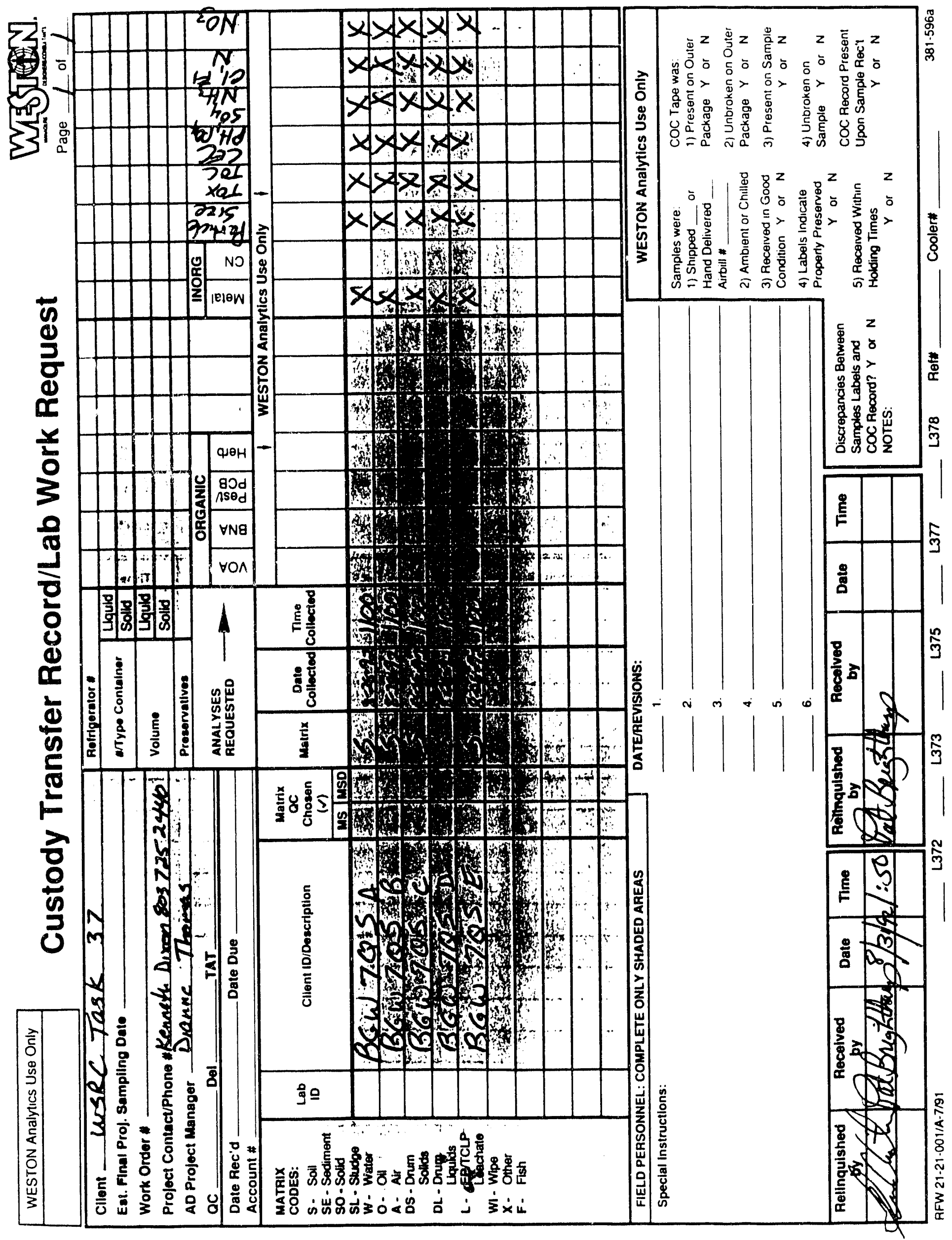




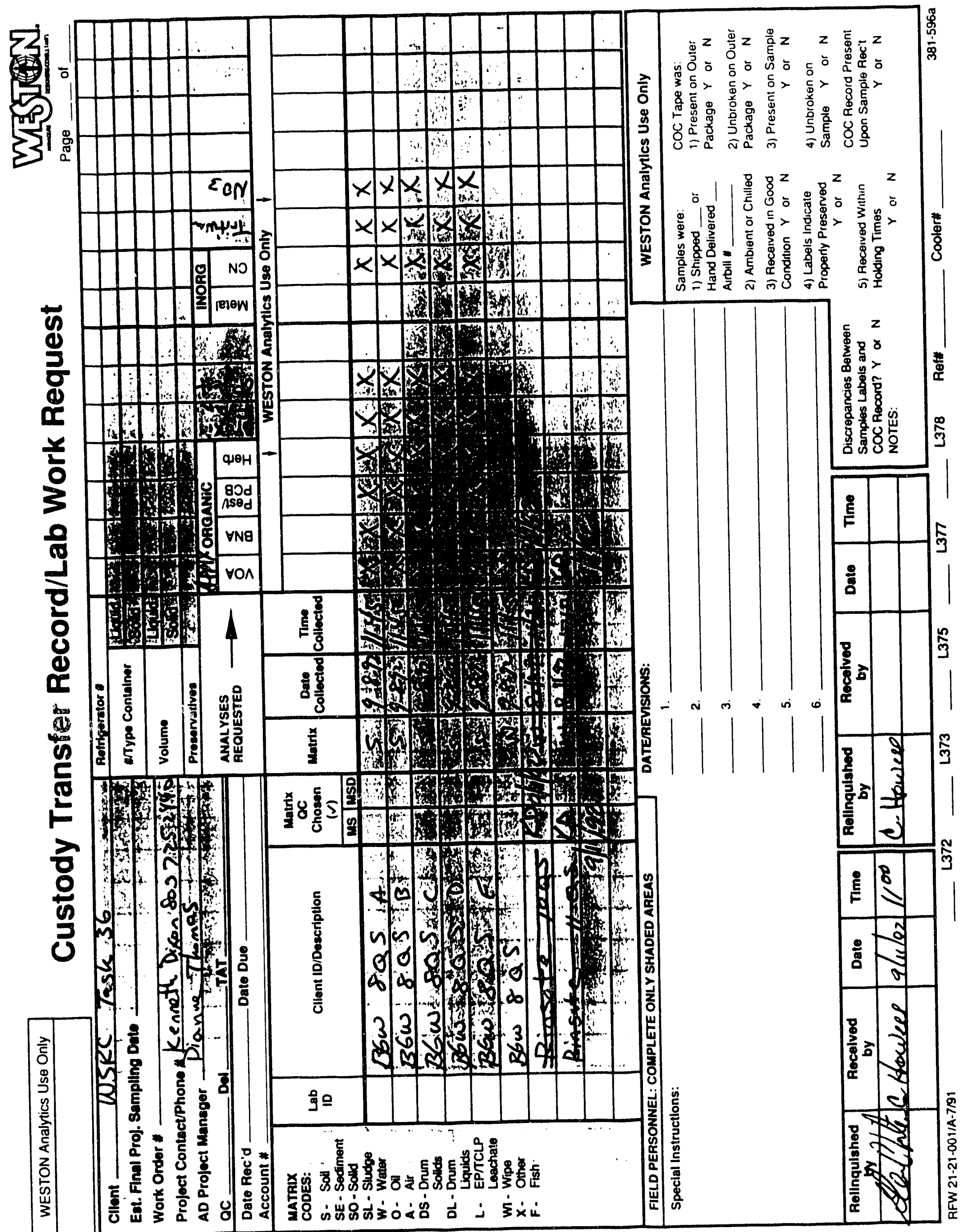




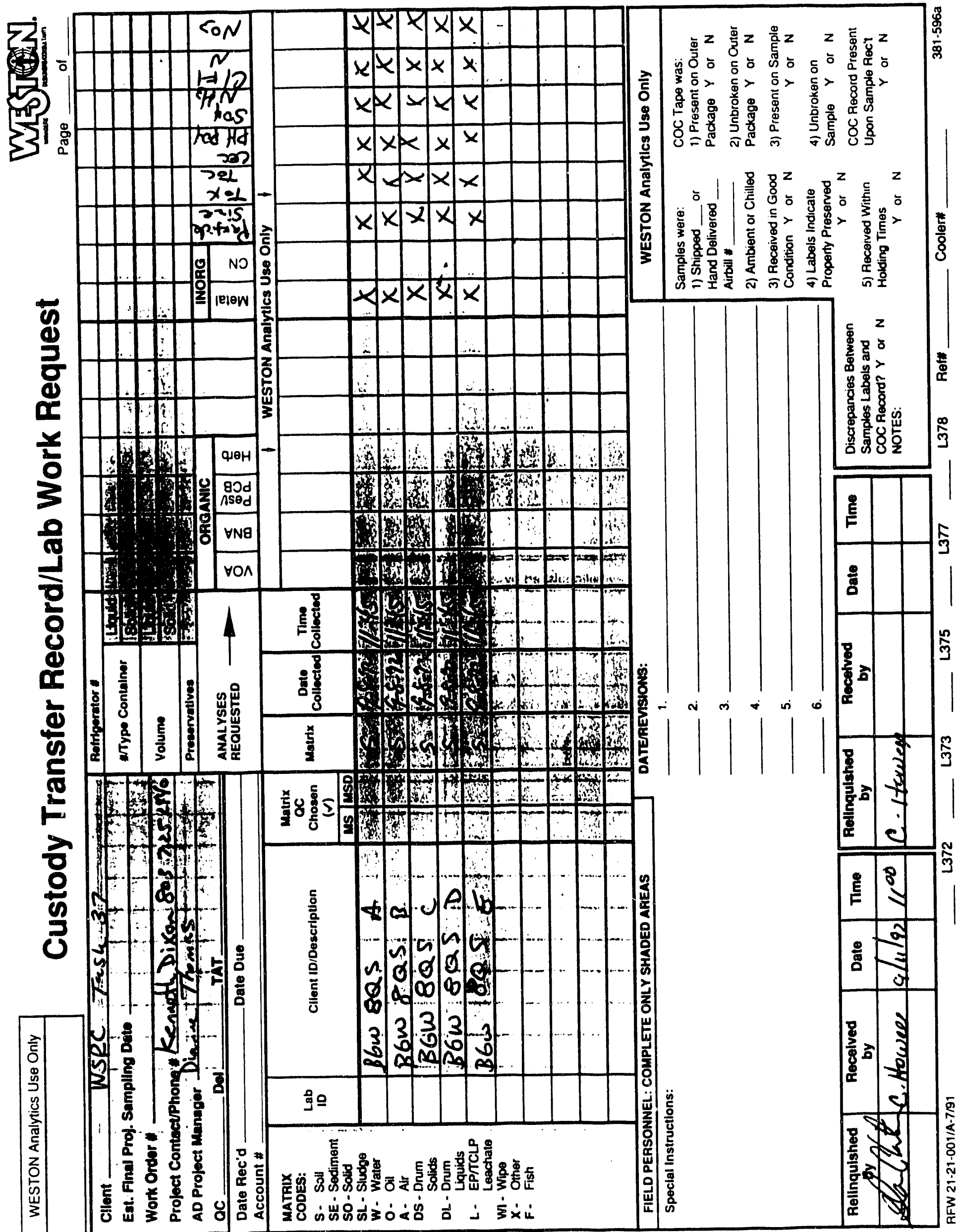


5
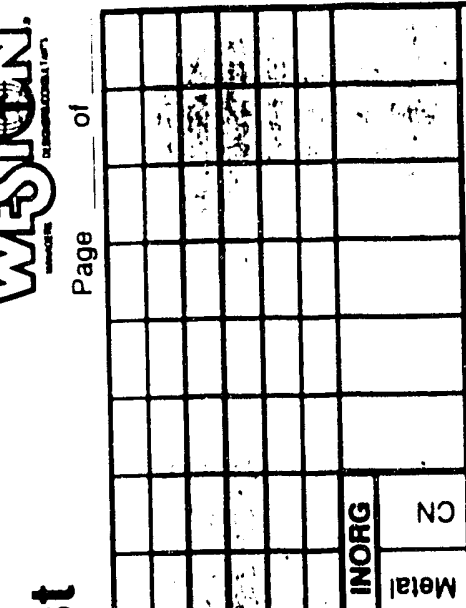

童
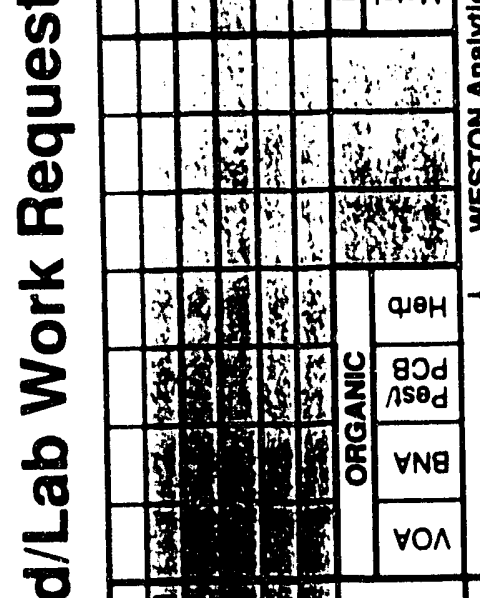

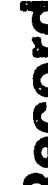

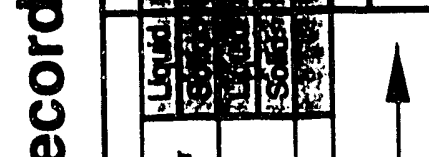

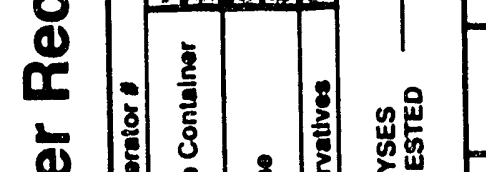

क

-1

7 (

$\frac{9}{6}+2$

$3 \sqrt{2}-20$

บ

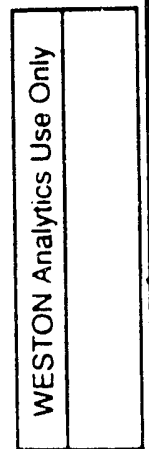

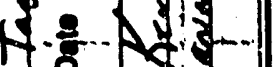

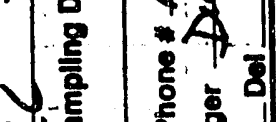

N.

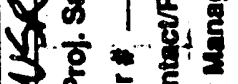

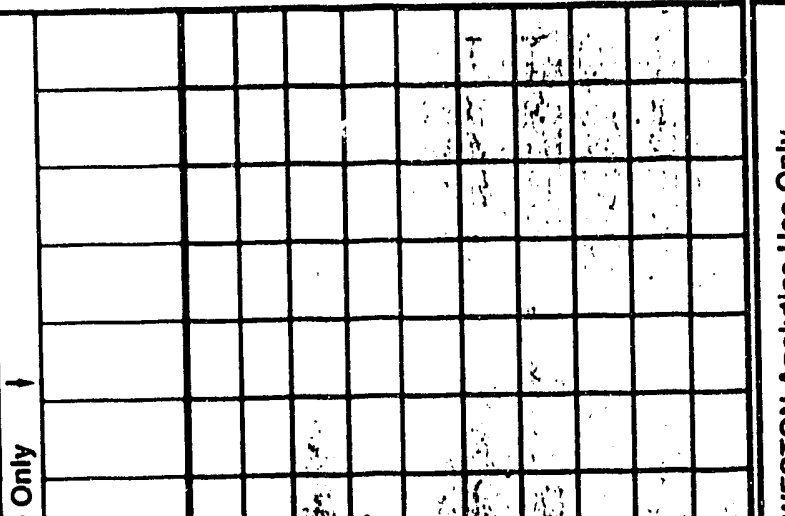

$\times \times$ W
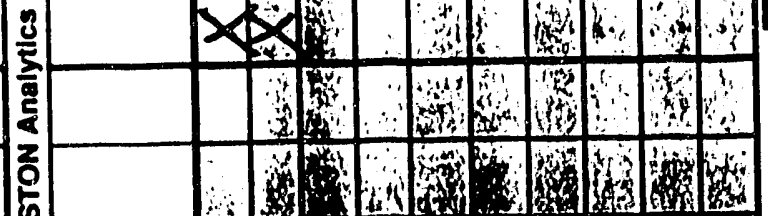

id

is.

Den

(4) 0 on

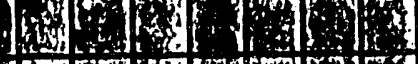

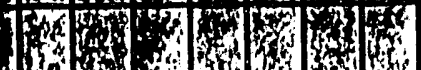

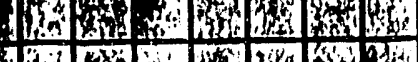

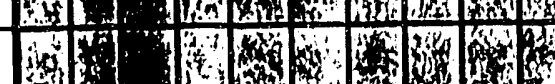

a.

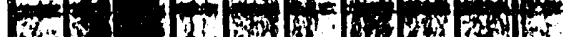

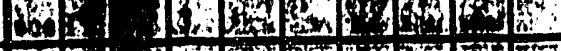

है

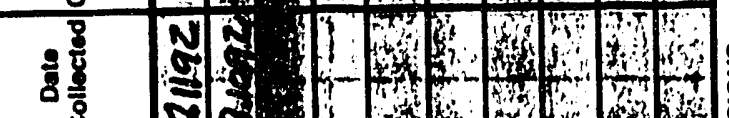

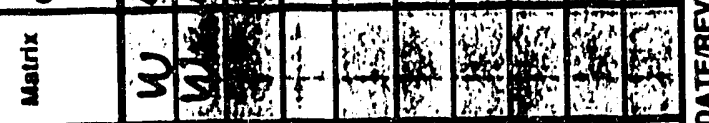

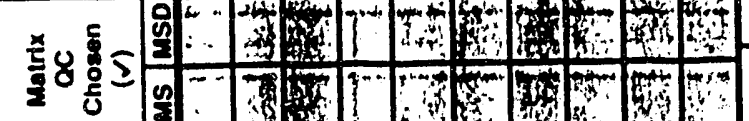

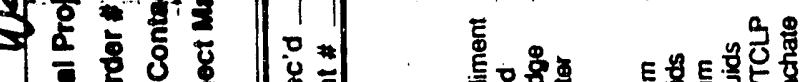

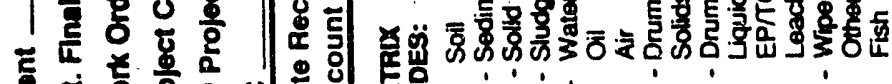

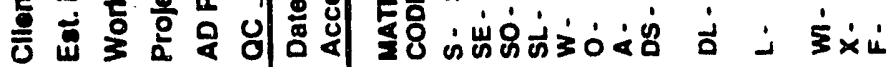

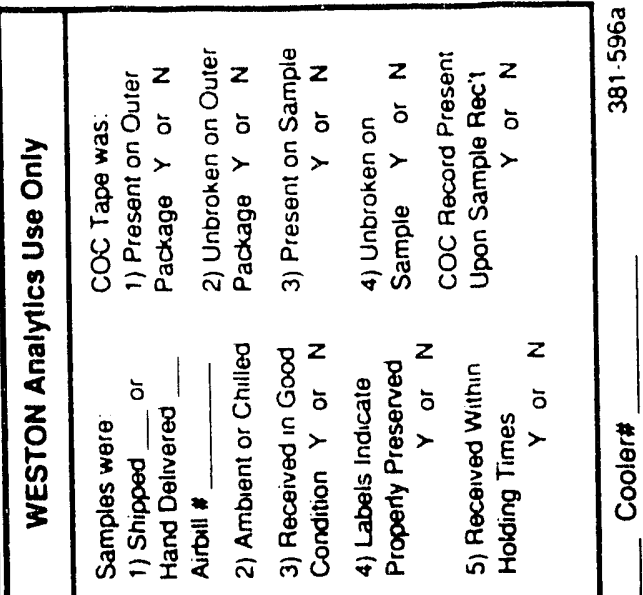

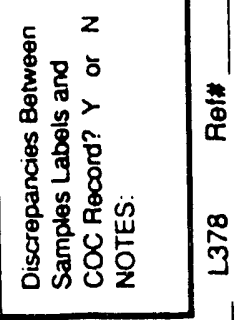

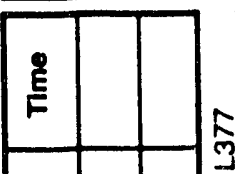

:

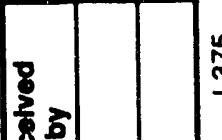

$\frac{3}{8}$

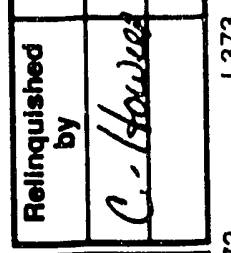

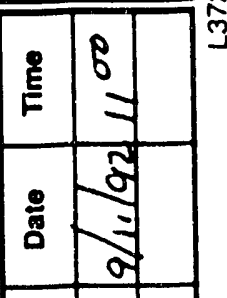

告 

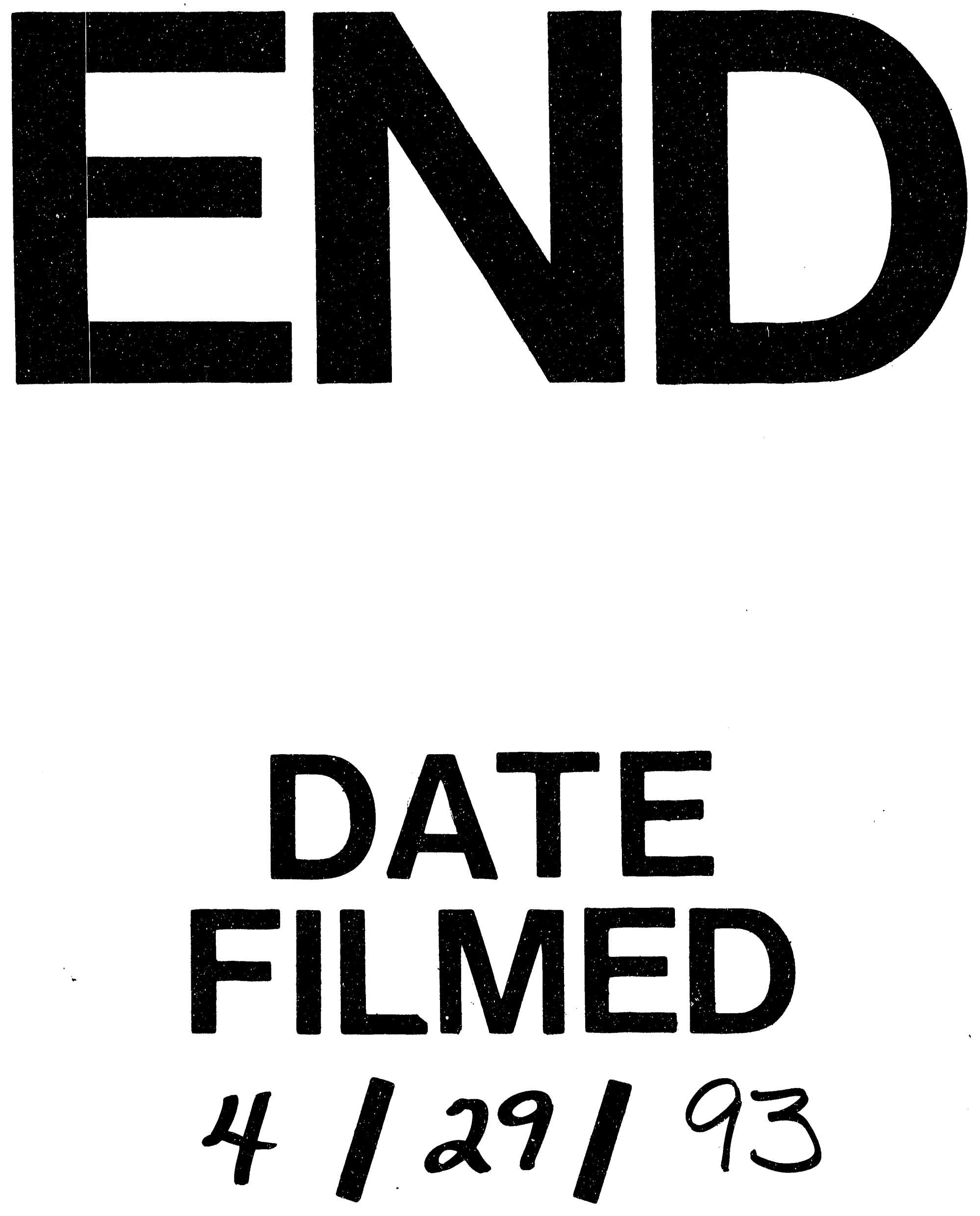

1 
